Liquid-Phase and Gas-Phase Modification of Silica Nanoparticles

for Dielectric Nanocomposites

Amirhossein Mahtabani 
LIQUID-PHASE AND GAS-PHASE MODIFICATION OF SILICA NANOPARTICLES FOR DIELECTRIC NANOCOMPOSITES

Amirhossein Mahtabani 


\title{
LIQUID-PHASE AND GAS-PHASE MODIFICATION OF SILICA NANOPARTICLES FOR DIELECTRIC NANOCOMPOSITES
}

\author{
DISSERTATION
}

to obtain

the degree of doctor at the Universiteit Twente,

on the authority of the rector magnificus,

prof. dr. ir. A. Veldkamp,

on account of the decision of the Doctorate Board

to be publicly defended

on Friday 22 October 2021 at 16.45 hours

by

Amirhossein Mahtabani

born on the 13th of January, 1990

in Ahvaz, Iran 
This dissertation has been approved by:

Supervisors

prof. dr. W. K. Dierkes

prof. dr. A. Blume

Cover design: Amirhossein Mahtabani

Printed by: Gildeprint, Enschede

Lay-out: Amirhossein Mahtabani

ISBN: 978-90-365-5274-5

DOI: $10.3990 / 1.9789036552745$

(C) 2021 Amirhossein Mahtabani, The Netherlands. All rights reserved. No parts of this thesis may be reproduced, stored in a retrieval system or transmitted in any form or by any means without permission of the author. Alle rechten voorbehouden. Niets uit deze uitgave mag worden vermenigvuldigd, in enige vorm of op enige wijze, zonder voorafgaande schriftelijke toestemming van de auteur. 


\section{VIT \\ UNIVERSITY \\ OF TWENTE.}

Tervakoski film «

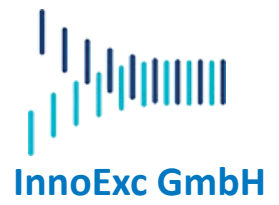

글 CTRONGON"

always in charge
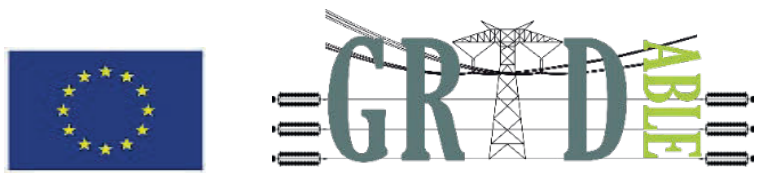

This project has received funding from the European Union's Horizon 2020 research and innovation program under grant agreement No. 720858.

\section{Graduation Committee:}

Chair / secretary:

Supervisors:

Committee Members: prof. dr. ir. H. F. J. M. Koopman

prof. dr. W. K. Dierkes

prof. dr. A. Blume

prof. dr. L. E. Govaert

prof. dr. F. R. Wurm

prof. dr. R. Ross

prof. dr. J. R. van Ommen

dr. T. Andritsch 


\section{TABLE OF CONTENTS}

\section{Chapter 1}

Introduction

References

\section{Chapter 2}

Literature Study

Dielectric Nanocomposites: A Quest for Understanding

1 Introduction. 18

2 The Nature of Charge Carriers in Polymers .................................................. 20

3 Charge Injection from Electrodes ................................................................ 21

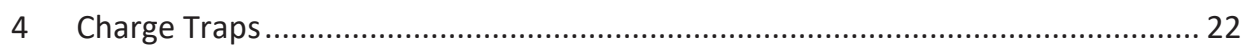

5 Nanodielectric Models.............................................................................. 23

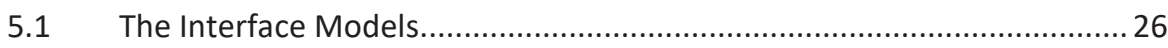

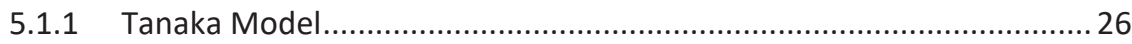

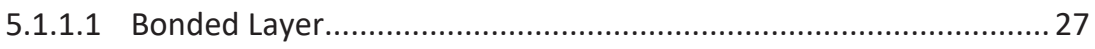

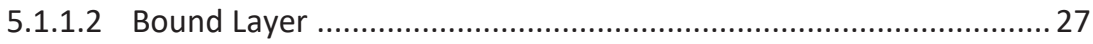

5.1.1.3 Loose Layer............................................................................. 28

5.1.1.4 Electric Double Layer................................................................ 28

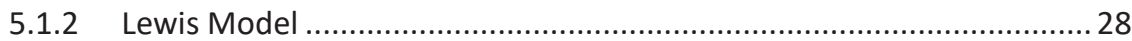

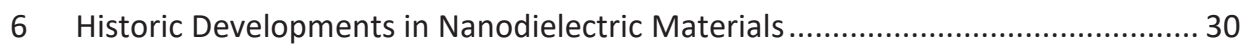

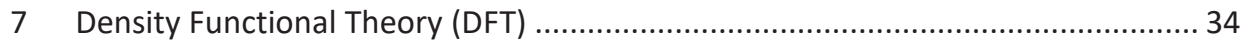

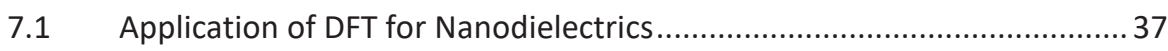

8 Surface Modification of Nanoparticles ................................................................ 39

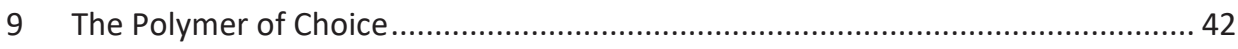

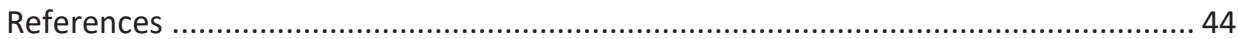

\section{Chapter 3}

Amino Functionalized Silica Nanoparticles in Polypropylene-Based Nanodielectrics

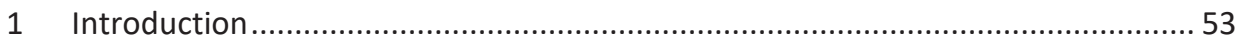

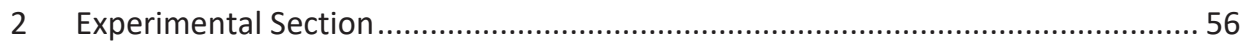

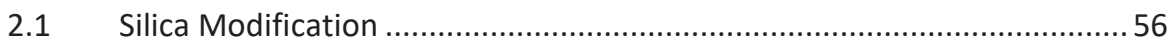


2.2 Preparation Of The Nanocomposites .................................................. 58

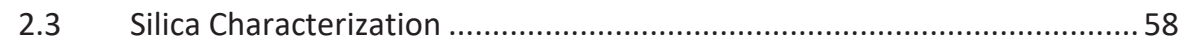

2.4 Thermally Stimulated Depolarization Current (TSDC) ............................. 59

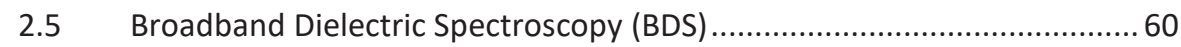

2.6 Morphology and Crystallinity Analysis ............................................ 60

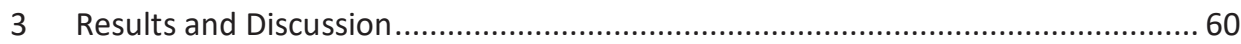

3.1 Characterization of Nanoparticles ......................................................6 60

3.2 Characterization of PP/EOC/Silica Nanocomposites ...............................66

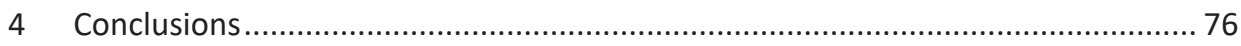

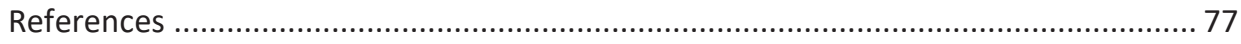

Chapter 4

Ureido and Methacrylate Functionalized Silica Nanoparticles in Polypropylene-Based Nanodielectrics ......................................................................................................81

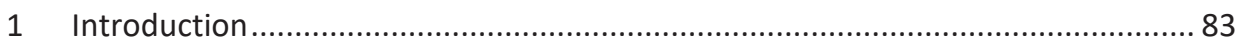

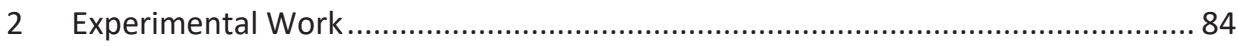

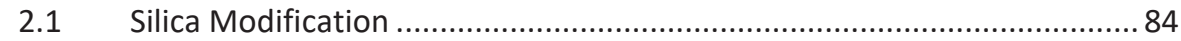

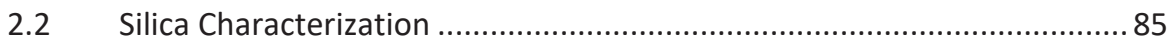

2.3 Preparation of The Nanocomposites..................................................... 85

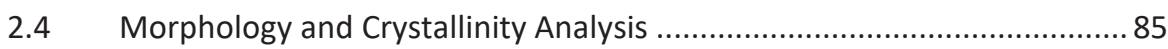

2.5 Thermally Stimulated Depolarization Current (TSDC) ............................. 85

2.6 Broadband Dielectric Spectroscopy (BDS) .......................................... 86

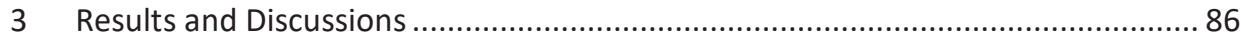

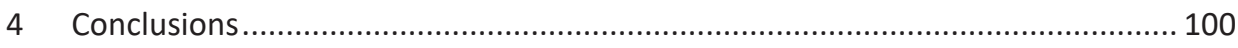

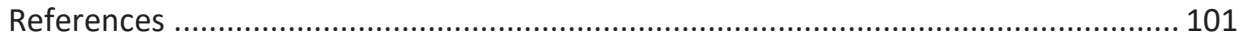

Chapter 5

Gas Phase Modification of Silica Nanoparticles in a Fluidized Bed...........................104

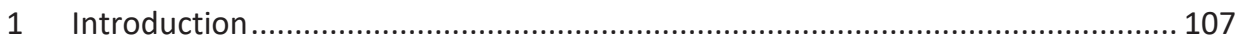

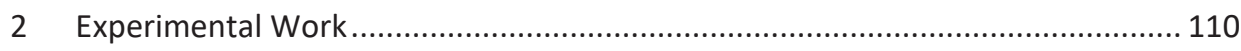

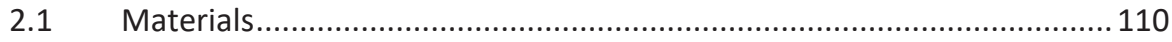

2.2 Gas Phase Deposition of The Silane ................................................ 110 
2.3 Characterization of The Nanoparticles ................................................ 112

2.3.1 Thermogravimetric Analysis (TGA).............................................. 112

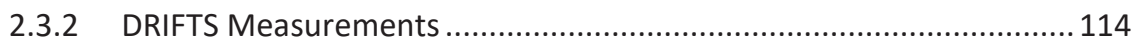

2.3.3 X-Ray Photoelectron Spectroscopy (XPS) ....................................... 114

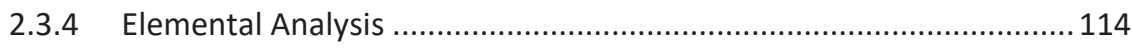

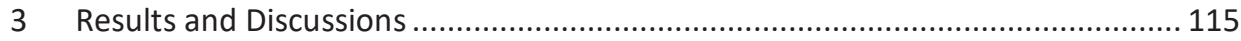

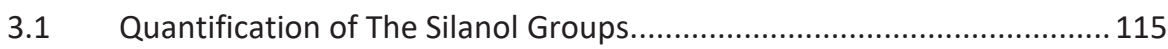

3.2 Gas-Phase Deposition of The Amino-Silane ....................................... 117

3.3 Repetition of Amino-Silane/Water Cycles .......................................... 124

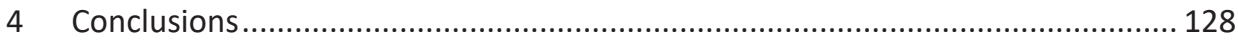

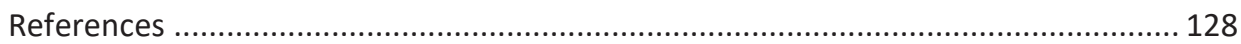

\section{Chapter 6}

Gas-Phase Modified Silica Nanoparticles in Polypropylene-Based Nanodielectrics

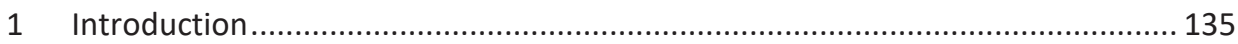

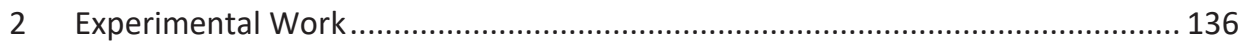

2.1 Silica Modification and Characterization............................................ 136

2.2 Preparation of The Nanocomposites................................................... 137

2.3 Morphology and Crystallinity Analysis ............................................. 137

2.4 Thermally Stimulated Depolarization Current (TSDC) .......................... 137

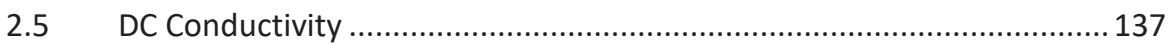

2.6 Broadband Dielectric Spectroscopy (BDS) ......................................... 138

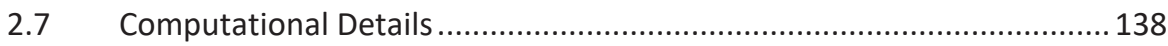

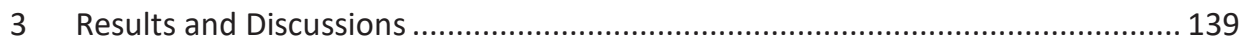

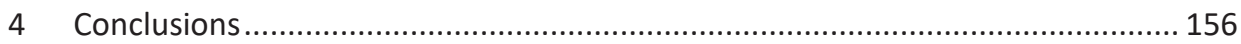

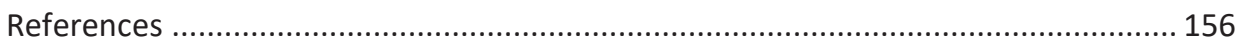

Chapter 7

Fluidized Bed Molecular Layer Deposition of Polyurea.......................................161

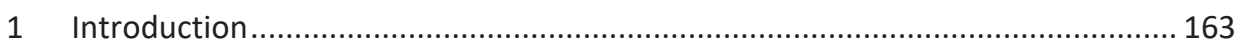

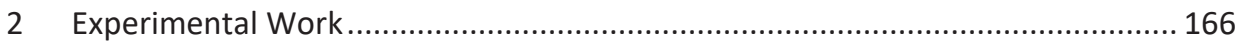




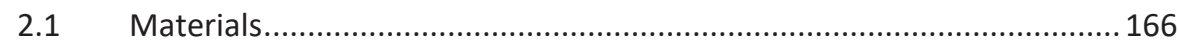

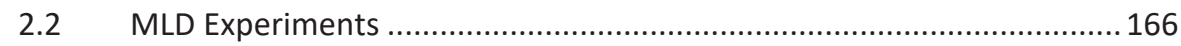

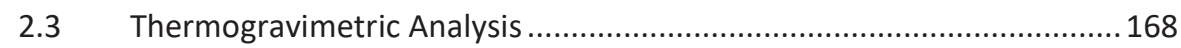

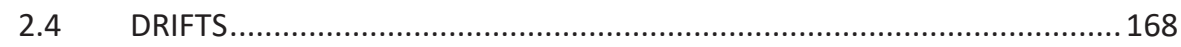

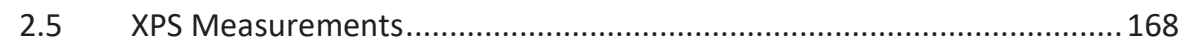

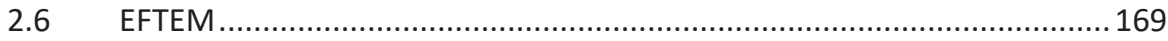

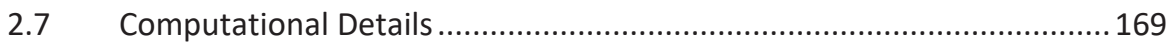

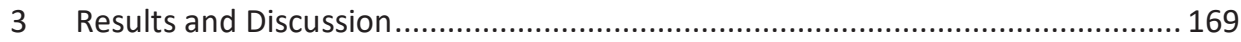

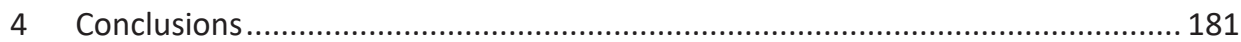

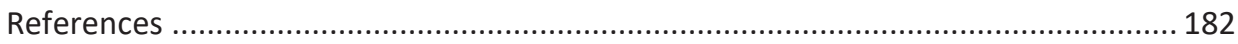

Chapter 8

Dielectric Properties of Nanocomposites: Effect of Molecular Layer Deposition of Polyurea on Silica Nanoparticles ...........................................................................185

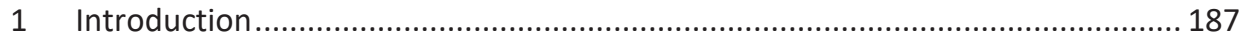

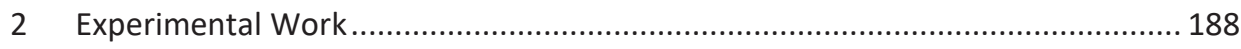

2.1 Silica Modification by Molecular Layer Deposition ............................... 188

1.1 Preparation of The Nanocomposites................................................. 188

2.2 Morphology and Crystallinity Analysis ............................................. 188

2.3 Thermally Stimulated Depolarization Current (TSDC) .......................... 188

2.4 Broadband Dielectric Spectroscopy (BDS) .......................................... 188

2.5 Computational Details .................................................................... 188

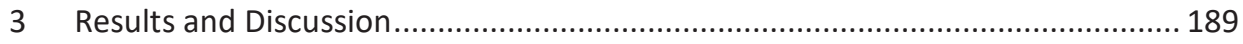

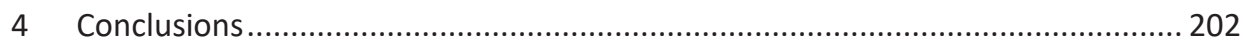

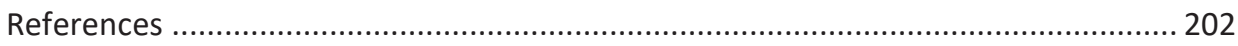

Chapter 9

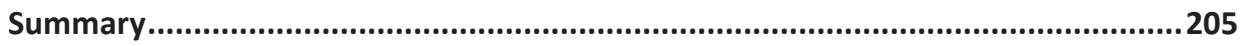

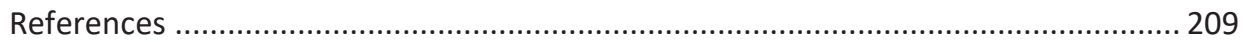

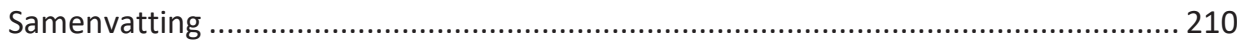

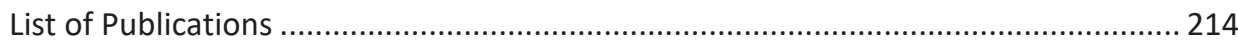

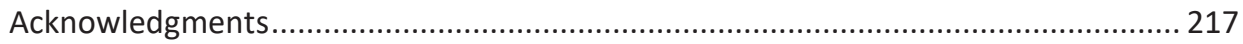


CHAPTER 1

Introduction 
Polymer nanocomposites with half a century of history are still one of the most interesting materials being extensively studied by scientists. After realizing the exceptional properties of nano-sized materials, polymer nanocomposites were industrially developed as engineering plastics in the early 1990s ${ }^{1}$. These materials are defined as the combination of a polymeric host material and a filler with at least one dimension below $100 \mathrm{~nm}$. By the addition of a few weight percent of a nanoscale filler to a polymer resin, numerous properties of the resulting material are significantly improved. These improvements are known to be related to the large interfacial areas imparted by the high surface area nanoparticles when dispersed well in the polymeric material ${ }^{2}$. This is the main advantage of nanocomposites over the conventional microcomposites. With this technology, it is possible to develop materials with superior properties depending on their application. Generally, inorganic nanofillers benefit from good optical, electrical, thermal and mechanical properties, while organic materials, including polymers, exhibit other excellent properties such as lightness, flexibility and processability. So, nanocomposites are expected to have complementary properties that neither the host matrix nor the added nanofiller alone shows. The appearance of polymer nanocomposites with dielectric and insulating properties dates back to the early $2000 \mathrm{~s}^{3}$. Since then, the research and development of nanocomposites with improved dielectric properties have led to the realization that these materials are, in fact, very good candidates for applications such as electrical insulation for power equipment, high voltage cables and electronic devices. The term nanodielectric is often used to describe polymer nanocomposites with dielectric applications, which is a recurring term in the upcoming chapters and serves as the main focus of the present thesis.

The promising potential of nanodielectrics has become particularly appealing to the high voltage cable industries, who have started to incorporate these materials into their production ${ }^{4}$. Despite this potential, however, industrialization of nanodielectrics suffers from the limited fundamental understanding of the underlying mechanisms determining their properties. Accordingly, cable industries together with research facilities are taking meticulous measures to leap towards a full realization of the nanodielectrics' potential. 
The project GRIDABLE is one these efforts ${ }^{5}$. Various industrial and academic partners have come together in a multidisciplinary consortium to address the industrial promise of nanodielectrics for the production of high voltage direct current (HVDC) cable insulation and capacitor films. The objective of this project is to put thermoplastic nanodielectrics to test on both, pilot and large scales, and select the most suitable candidates for production. The development of HVDC technology, is essentially directed at the production and transmission of green energy ${ }^{6}$. The appealing idea of a super grid, to transmit green energies from where it is abundant, over long trans-continental distances has been around for several years (see Figure 1.1). In the path towards this milestone, it is necessary to maintain the sustainable attitude down to the infrastructure level. Yet, most of the existing HVDC grids are equipped with insulation systems based on crosslinked polyethylene (XLPE) ${ }^{7}$. Despite its good electrical properties, polyethylene (PE) has to be chemically crosslinked in order to obtain the mechanical properties suitable for HVDC cable insulation applications. This would result in a material that is not recyclable, and hence, not sustainable. As an effort to address this issue, GRIDABLE focuses on polypropylene (PP) based nanodielectrics for HVDC application. PP has proven to possess high dielectric strength, comparable to PE, and due to the absence of crosslinks, it can be recycled and re-used for other low demanding applications ${ }^{8}$. Exhibiting satisfactory electrical properties, PP appears as a sustainable and promising candidate to replace XLPE in HVDC technology. The lower mechanical properties of PP compared to XLPE can be effectively compensated by blending it with polyolefin elastomers, ethylene propylene diene rubber (EPDM) or linear low-density PE (LLDPE) ${ }^{9-10}$. As to the choice of the nano-filler, it is important to use an active treatable particle with high purity and minimal moisture content. The treatability of the nanoparticles enables one to modify their surface properties to achieve improved dispersion and tailor their interfacial interactions with the surrounding polymer chains. Fumed silica nanoparticles, offering large treatable surface areas, low contamination and moisture contents are excellent candidates for developing high performance nanodielectrics. Along with these characteristics, fumed silica nanoparticles also exhibit high electron-phonon interactions ${ }^{11-12}$, i.e. they can interact with charge carriers inside the nanodielectric, immobilize them and likely contribute to the enhancement of the dielectric properties of the material. 


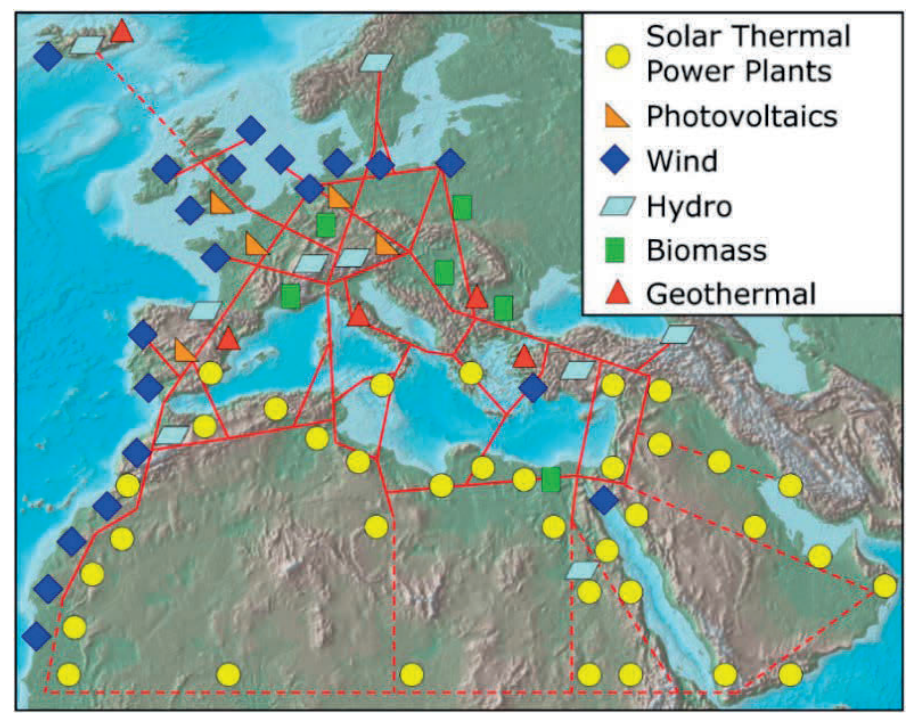

Figure 1.1. A conceptual super grid linking renewable resources across North Africa, the Middle East and Europe ${ }^{13}$

The superior properties of nanodielectrics lie within the large interfacial areas induced by the nanoscale fillers in the polymeric matrix: a complex spatially extensive region where the material exhibits properties that are neither of the filler nor the polymer matrix. These interfacial areas ultimately determine the macroscopic dielectric properties of the nanocomposites. In fact, a deep physical and chemical understanding of these interfaces is of utmost importance in order to tailor the properties of the nanodielectrics and develop high performance insulation systems. Tailoring the characteristics of the interfacial areas in nanodielectrics is the main theme of the present thesis.

One of the most practical approaches towards this objective is through modification of the filler surface. By introducing new functional groups onto the surface of nanoparticles, it is possible to tailor the interactions in the interfacial zones, and ultimately, control the dielectric properties of the nanocomposite. Three different approaches for nanoparticle modification are presented and discussed in the framework of this thesis. The conventional liquid-phase treatment where the modification takes place in a solution is a relatively straight forward method which utilizes a wet chemistry approach to modify the nanoparticle surfaces. Nevertheless, to address the shortcomings of the solution technique 
such as the recovery of solvents, wet extraction of by-products and lack of control over the morphology and thickness of the deposited layer, two novel gas-phase methods are also introduced and studied in this work: atomic and molecular layer deposition (ALD and MLD) are utilized for gas-phase modification of the nanoparticles in a fluidized bed. These methods do not suffer from the shortcomings of the wet process and offer outstanding control over the morphology and conformity of the modification layer. Since the gas-phase methods are developed for the first time in this study, separate chapters are dedicated to elaborate on the details of the processes and characterization of the resulting nanoparticles.

In Chapter 2, we first introduce the concept of nanodielectrics, and discuss the models describing the characteristics of the interfacial areas. Chapter 2 serves as a literature survey, a quest for understanding the dielectric phenomena at the nanometric scale.

Chapter 3 discusses the liquid-phase modification of silica nanoparticles with 3aminopropyltriethoxysilane (APTES) and its effect on the dielectric properties of PP based nanodielectrics. In particular, we investigate the influence of this type of modification on the charge trapping and transport phenomena in the studied nanocomposites.

Chapter 4 is also dedicated to the solution modification method, using 3(trimethoxysilyl)propyl methacrylate (TMPM) and 1-[3-(trimethoxysilyl)propyl]urea (TMPU) as modifying agents, to introduce ureido and methacrylate functional groups onto the silica nanoparticles. Subsequently, the effect of such surface treatment is studied on the dielectric response of the nanocomposites.

In Chapter 5 we introduce a novel gas-phase method, similar to atomic layer deposition (ALD), to deposit layers of aminopropylsiloxane onto the silica nanoparticles in a fluidized bed using APTES as the precursor. The modification process is investigated in detail, and the resulting nanoparticles are characterized by several experimental methods.

Chapter 6 focuses on the dielectric properties of nanocomposites filled with the APTES-modified nanoparticles in the gas-phase. Firstly, we demonstrate the alterations in the electronic structure of the silica surface upon this type of modification by means of density functional theory (DFT) modeling. Then, the effect of this self-limiting controlled 
modification method on the charge trapping properties of the nanodielectrics is investigated and compared to the model predictions.

The second gas-phase modification method is described in Chapter 7. Using phenyldiisocyanate (PDIC) and ethylenediamine (ED) as the precursors, consecutive layers of polyurea are deposited onto the silica surface via molecular layer deposition (MLD) in a fluidized bed for the first time. The process is described in detail and the resulting nanoparticles are characterized by means of several techniques.

The dielectric performance of the nanocomposites filled with the MLD-modified nanoparticles is studied in Chapter 8. The DFT method is also utilized in this chapter in order to realize the electronic structure of these functionalized nanoparticles in the presence of an external electric field. The model predictions are then validated by experimental results. We demonstrate the introduction of new localized states resulting from the polyurea repeating units and elaborate on their influence on the charge trapping and transport phenomena in the studied nanodielectrics.

Finally, a global summary is presented in Chapter 9. Final conclusions, additional remarks and implications of the presented results in this study are summarized in this chapter, and a possible future roadmap is described.

As mentioned in the beginning of this chapter, the underlying mechanisms through which nanodielectrics impart their outstanding properties are still far from being fully understood, as there still exist numerous unknown unknowns. The ultimate objective of this work, therefore, is to provide more evidence of these phenomena, by focusing on the role of the nanoparticles and their surface properties, as a trial to unravel the potentialities of nanocomposites as insulation materials. Hopefully, the present thesis can be a stepping stone towards approaching this ultimate goal. 


\section{References}

[1] Deguchi, R.; Nishio, T.; Okada, A. High rigidity and impact resistance resin composition, Toyota Motor Corp., Ube Industries Ltd., Toyota Central R\&D Labs Inc., US5164440A, 1992.

[2] Lewis, T., Interfaces: nanometric dielectrics. Journal of Physics D: Applied Physics, 38 (2), 2005, p 202.

[3] Tanaka, T.; Imai, T., Advanced nanodielectrics: fundamentals and applications CRC press: 2017;

[4] Murata, Y.; Sakamaki, M.; Abe, K.; Inoue, Y.; Mashio, S.; Kashiyama, S.; Matsunaga, O.; Igi, T.; Watanabe, M.; Asai, S., Development of high voltage DC-XLPE cable system. SEI Technical Review, 101, 2013, pp 55-62.

[5] Project Gridable. http://www.gridable.eu/ (accessed 22/09/2021).

[6] Feltes, J. W.; Gemmell, B. D.; Retzmann, D. From Smart Grid to Super Grid: Solutions with HVDC and FACTS for grid access of renewable energy sources, 2011 IEEE Power and Energy Society General Meeting, 2011; pp 1-6.

[7] Vrana, T. K.; Energi, S. Review of HVDC component ratings: XLPE cables and VSC converters, 2016 IEEE International Energy Conference (ENERGYCON), IEEE: 2016; pp 1-6.

[8] Gao, Y.; Huang, X.; Min, D.; Li, S.; Jiang, P., Recyclable Dielectric Polymer Nanocomposites with Voltage Stabilizer Interface: Toward New Generation of High Voltage Direct Current Cable Insulation. ACS Sustainable Chemistry \& Engineering, 7 (1), 2019, pp 513-525.

[9] Kurahashi, K.; Matsuda, Y.; Ueda, A.; Demura, T.; Miyashita, Y.; Yoshino, K. The application of novel polypropylene to the insulation of electric power cable, IEEE/PES Transmission and Distribution Conference and Exhibition, IEEE: 2002; pp 1278-1283.

[10] Du, B. X.; Xu, H.; Li, J.; Li, Z., Space charge behaviors of PP/POE/ZnO nanocomposites for HVDC cables. IEEE Transactions on Dielectrics and Electrical Insulation, 23 (5), 2016, pp 3165-3174.

[11] Shi, N. Properties of nanoscale dielectrics from first principles computations. PhD Thesis, University of Connecticut, 2008.

[12] DiStefano, T.; Shatzkes, M., Impact ionization model for dielectric instability and breakdown. Applied Physics Letters, 25 (12), 1974, pp 685-687.

[13] DESERTEC Foundation, Energy for the next billion. www.desertec.org (accessed 22/09/2021). 



\section{CHAPTER 2}

Literature Study 


\section{Dielectric Nanocomposites: A Quest for Understanding}

\section{Introduction}

Polymer nanocomposites are hybrid materials where a small weight percent (wt.\%) of nanostructured fillers is incorporated into a polymeric matrix to achieve an improved performance in a wide range of applications ${ }^{1-3}$. In the 1990 s, polyamide/layered silicate nanocomposites were first commercialized as high impact, heat resistant materials ${ }^{4}$. Since then, the use of high performance nanocomposites has been a recurring theme for many studies $^{5-8}$. Dielectric nanocomposites were not an exception, as they comprise one of the major research topics in the fields of material, electrical and electronic engineering. The multidisciplinary nature of this field of research has attracted a large number of scientists, over the last two decades, to study these interesting materials ${ }^{9}$. This chapter aims to provide an extensive overview on the background, descriptive models and the recent developments in the field of polymer nanodielectrics. One tool for understanding the underlying phenomena in nanodielectrics and further development of this material class is density functional theory (DFT) which will also be introduced in this chapter.

In the realm of dielectric materials, the year 1994 is iconic, when Lewis introduced the concept of nanometric dielectrics, and elaborated the charge transfer mechanisms at the interfacial regions between two phases ${ }^{10}$. Starting with a metal electrode in contact with an insulator in the presence of an external electric field, he pointed out that the interface between the two phases is a nanometric system with properties which are invariably different from those of the bulk phases on either side. This layer extends from sub-atomic distances in the metal bulk to around $100 \mathrm{~nm}$ in the insulator phase, and is charged due to the presence of the electric field. To attain an equilibrium steady state, charges would have to be transported towards or away from this interface. The rate of this transfer, and the attainment of the steady state depend on the electrical conductivities of the two phases, and can be exceedingly long for insulating materials. The situation at the electrode/insulator interface can be illustrated as Figure 2.1. On the metal side, a quasi-free electron cloud is formed over less than an atomic distance. Chemical and physical forces will then drive 
dipoles, ions and other polar species in the insulator to form a monolayer, which is called the inner Helmholtz layer ${ }^{11}$. Beyond this, in the outer Helmholtz layer, species would be under the influence of both the inner layer and the forces in the bulk of the dielectric. Next to the Helmholtz layers in the dielectric is usually a diffuse layer, also known as GouyChapman space charge layer ${ }^{12-13}$, the extent of which depends on the concentration of the charged species in the dielectric material. This situation leads to a process of charge separation between the metal and the dielectric, with a charge density $q$ (also known as the image charge) residing in the metal boundary, and a counter charge $-q$ distributed in the Helmholtz and Gouy-Chapman layers. This $q$, $-q$ system forms a double layer, characteristic of the interface between a metal electrode and a dielectric insulator.

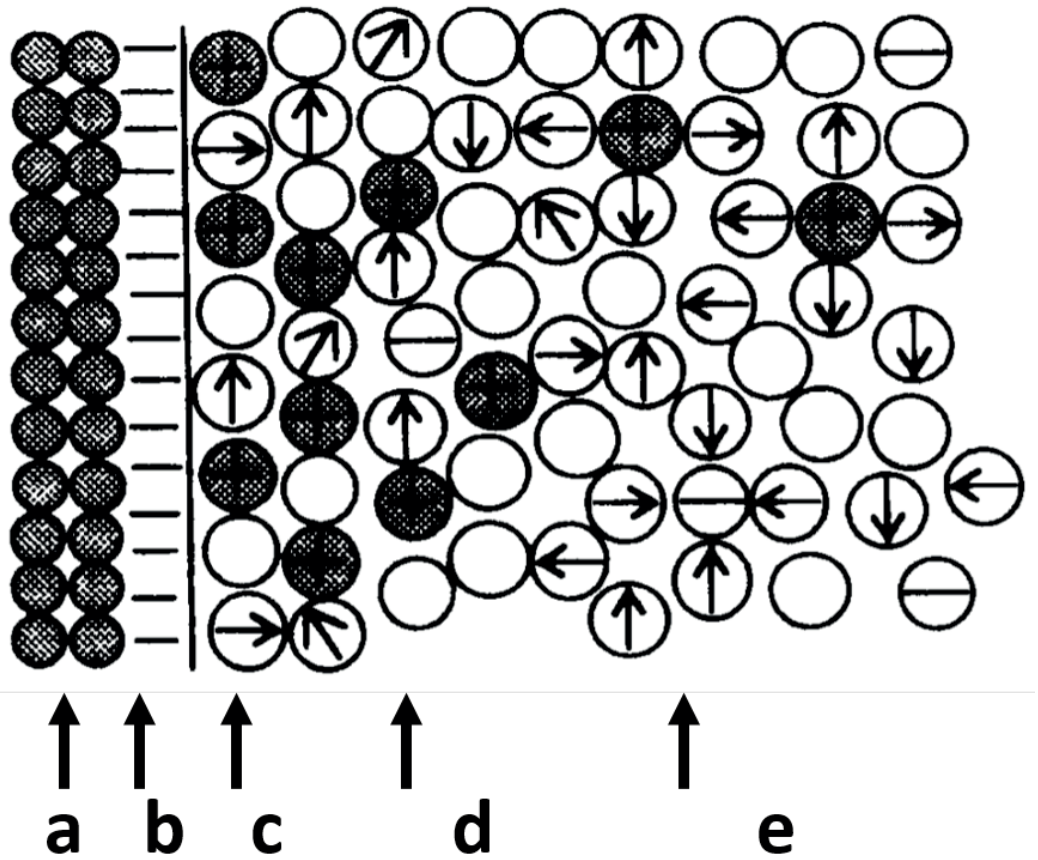

Figure 2.1. A metal electrode/dielectric interface depicting a: positive metal cores; b: the outer electron cloud of the metal; c: the structured inner Helmholtz layer of molecules and ions; $d$ : the outer Helmholtz layer and e: the Gouy-Chapman diffuse space charge layer. In this illustration the net charge in the dielectric $(-q)$ is considered positive, therefore, the image charge in the metal $(q)$ is negative ${ }^{10}$. 


\section{The Nature of Charge Carriers in Polymers}

Whether to assume that the charge carriers in polymeric materials are of ionic or electronic nature has certain implications when discussing charge transport in dielectrics. In general, the three possible sources of charge carriers are thermal ionization in the bulk material, ionization of impurities and field induced injection of electrons from an electrode ${ }^{14}$. While intramolecular charge motion, i.e. charge motion within the molecular structure of the polymer is known to be due to electrons or holes ${ }^{15}$, the intermolecular transfer is subject of debate ${ }^{16-17}$. Wintle ${ }^{14,17}$ states that it is unavoidable that the electronic charges interact with low molecular weight species in the bulk material and form ionic carriers. Therefore, he ascribes charge transport in technical insulators, whether gas, liquid or solid, to be largely characterized by ionic flow. However, the study of DC conductivity in dielectrics reveal that electronic charge injection plays a significant role in the mechanisms of charge transport. In general, the flowing current in a dielectric after a DC field is abruptly removed (or applied), is given by the Curie-von Schweidler law ${ }^{18}$ :

$$
I(t)=A t^{-n}
$$

(Equation 2-1)

Where $A$ is a constant ${ }^{19-21}, 0<n<1$ for most dielectrics, $t$ is time and voltage is applied/removed at $t=0$. It has been observed that after the voltage is applied, the current decays very slowly to a nearly constant value ${ }^{22-23}$. This constant current can be in the order of $10^{-8} \mathrm{~A}$ for planar $150 \mu \mathrm{m}$ polyethylene samples under an applied DC field of $20 \mathrm{kV} / \mathrm{mm}^{24-}$ ${ }^{25}$. The fact that this current remains nearly constant for long periods of time implies that there is an inexhaustible source of charge carriers in the material, despite the limited number of ionized species moving from one electrode and neutralizing at the other. Therefore, it can be assumed that given enough time, the steady-state DC conductivity and space charge distribution in polymeric materials is fairly governed by electronic charge transport. The same assumption will be held throughout this chapter to explain charge trapping and transport in nanodielectrics. 


\section{Charge Injection From Electrodes}

Charge injection across the interface between a dielectric material and a metallic electrode has been studied extensively for many years. There are two main models generally applicable when electric fields less than $10^{9} \mathrm{~V} / \mathrm{m}$ are applied:

i. The Schottky model ${ }^{26}$ which considers charge injection as field-assisted thermal excitation of an electron from the electrode to the bottom of the conduction band in the dielectric. According to this model, the electron exiting the electrode is subjected to two opposite forces: The image force, applied by the image layer in the electrode, drawing the electron back, and the force due to the electric field, which pushes the electron into the dielectric. These forces are functions of the electric field and the relative permittivity of the dielectric.

ii. The surface state tunneling model ${ }^{27}$ treats charge transfer as a tunneling process between the surface states of the electrode and the dielectric. Both the electrode and the dielectric possess donor and acceptor surface states. In the dielectric, these states are associated with dangling bonds and absorbed impurities on its surface. If the donor energy level is higher, or the acceptor energy level is lower than the Fermi level of the electrode, charge transfer may occur provided the tunneling distance is not too large. This kind of transfer does not occur uniformly over the interfacial area, as the contact between the two surfaces is not uniform on a molecular scale.

Upon transfer to the dielectric phase, electrons and holes are to move rapidly through the intra-chain states in the conduction and valence bands, respectively. Accordingly, the minimum mobility of the charge $\left(\mu_{\min }\right)$ transported through the band can be calculated from Equation 2-2:

$$
\mu_{\min }=\pi e a^{2} / 4 h
$$

(Equation 2-2)

Where $e$ is the electron charge, $a$ is the lattice spacing in the dielectric, and $h$ is Planck's constant ${ }^{28}$. In case of a polymeric dielectric, the lattice spacing is the distance between two repeating units in the chain. 
For instance, considering $a=0.254 \mathrm{~nm}$ for polyethylene $(\mathrm{PE})^{29}$, Equation 2-2 yields a $\mu_{\min }$ of $1.2 \times 10^{-5} \mathrm{~m}^{2} \mathrm{~V}^{-1} \mathrm{~s}^{-1}$, which is orders of magnitude higher than the experimentally measured charge mobility for insulating polymers, mostly in the range of $10^{-13}-10^{-15} \mathrm{~m}^{2} \mathrm{~V}^{-1} \mathrm{~s}^{-1}$ at room or slightly higher temperatures ${ }^{30-32}$. This significant difference suggests that charge transport in dielectrics is strongly affected by trapping. An electron may be moving rapidly through the conduction band for a short time, but its mobility significantly reduces as it gets immobilized for much longer periods in localized states i.e. traps.

\section{Charge Traps}

Ideally, each atom in the material's periodic crystal lattice should be located at the appropriate position, so that the resulting structure has the lowest level of energy ${ }^{33}$. Any deviation from this situation would lead to defect points with localized energy states i.e. traps. These states are localized, that is, they exist only in defect points of the material structure, where the continuity of the lattice is disturbed. In polymers, traps are formed by the conformational defects in the polymer chains ${ }^{34}$, and by the presence of chemical defects such as crosslinks, contaminations (e.g. by-products of crosslink reactions or filler treatment), additives (fillers, antioxidants etc.), branches and double bonds along the polymer backbone. The traps created by the conformational defects of the polymer chains are not permanent as they may be disintegrated when temperature approaches the onset of certain chain segment motions such as crankshaft motions (around $-120{ }^{\circ} \mathrm{C}$ for polyethylene) ${ }^{35}$. However, if the trap is permanent, the electron may only escape when it has gained sufficient energy to surmount the potential well, presented by the trap. The electron then moves freely through the conduction band until it is captured by another localized state. This is illustrated in Figure 2.2, where electron transfer occurs by thermally assisted resonance tunneling from Site 1 to Site $2^{36-37}$. In this case, the electron in Site 1 at energy $E_{a 1}$ is thermally excited to an energy level $E$ followed by tunneling through the potential barrier $E(x)$, to an equivalent level in Site 2, and de-excites to energy $E_{a 2}$. 


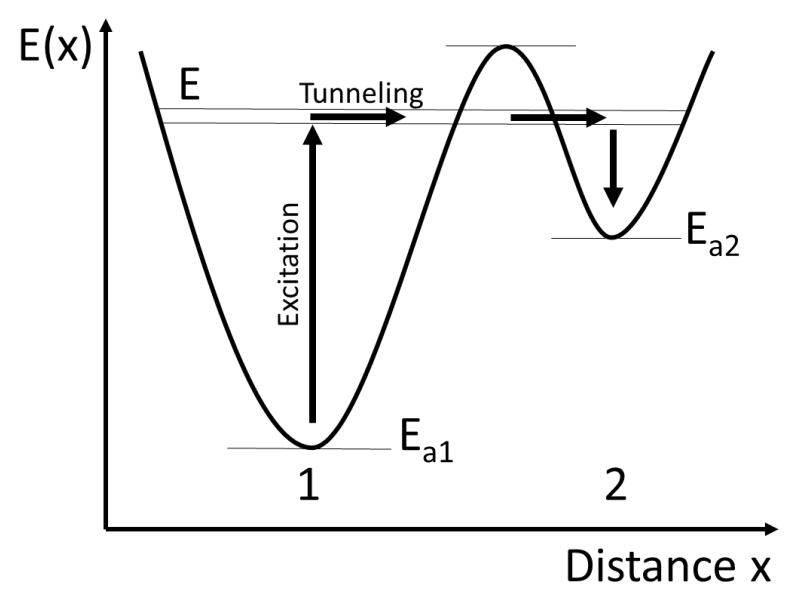

Figure 2.2. Schematic presentation of thermally assisted resonance tunneling of an electron between two traps ${ }^{36}$.

\section{Nanodielectric Models}

Polymer nanocomposites are distinctive, in the sense that they are endowed with significantly larger interfacial surface areas compared to the conventional microcomposites. For polymeric nanodielectrics, the study of these interfacial regions has led to developments of models describing their characteristics. Yet before describing these models, let us first focus on the interface itself and its physical properties.

At the molecular level, the influence of polymer/filler interactions on the molecular mobility and structure of the adjacent polymer chains are signified when the filler dimensions are in the nanometric range. Experimental work has shown a reduction in molecular mobility of the polymer chains at the interfacial region ${ }^{38}$. For polymer chains near the solid surface of the nanoparticle (NP), the number of possible configurations, and hence, the configurational entropy decreases ${ }^{39}$. This would induce an entropic force leading the chains to recoil from the surface. This results in a layer of polymer chains with a reduced density adjacent to the NP. Figure 2.3a presents the mass density $\rho(R)$ of polyethylene (PE) as a function of its distance from a silica NP, compared to the neat matrix simulated at different conditions ${ }^{40}$. The silica NP occupies the space up to $\mathrm{R} \sim 22 \AA$. From this point and 
within intervals of $\sim 4 \AA$ the peak mass densities represent polymer shells around the NP. When there is no strong interaction e.g. chemical bonding between the polymer and filler, chains tend to be aligned parallel to the NP surface. This is well demonstrated by Brown et al. for a PE/silica interface ${ }^{40}$. The angle $\theta$ is defined as that between the outward vector normal to the interface and the local axis of the polymer chain. Accordingly, the correlation between $\theta$ and the distance from the center of the NP (R) can be explained by the solvation of two Legendre functions $P_{1}(\cos \theta)$ and $P_{2}(\cos \theta)$ defined as

$$
\begin{array}{cc}
P_{1}(\cos \theta)=\langle\cos \theta\rangle & \text { (Equation 2-3) } \\
P_{2}(\cos \theta)=\frac{3}{2}\left\langle\cos ^{2} \theta\right\rangle-\frac{1}{2} & \text { (Equation 2-4) }
\end{array}
$$

$\theta$ and $\mathrm{R}$ are related via simple vector math. $\theta$ is basically the angle between two vectors: one connecting the center of the particle to the monomer $\mathrm{i}$ (in the polymer chain), and the other connecting monomer $\mathrm{i}$ to monomer $\mathrm{i}+1$. The sum of these two vectors, gives R. $P_{1}(\cos \theta)$ is normally zero due to the symmetry of the model, and has minimal implications. The variation of $P_{2}(\cos \theta)$ with $\mathrm{R}$, however, has insightful indications. $P_{2}(\cos \theta)$ is $-1 / 2$ for a perfectly perpendicular alignment, 1 for perfectly parallel alignment and 0 for a random alignment. It is clear in Figure $2.3 \mathrm{~b}$ that in the vicinity of the NP $(R \sim 22 \AA$ ), polymer chains are perpendicular to the interface normal vector; i.e. the chains are parallel to the interface. As $\mathrm{R}$ increases, $P_{2}(\cos \theta)$ oscillates and gradually tends to 0 which indicates the random alignment of the polymer chains at sufficiently large distances from the NP surface. The mass density $\rho(R)$ and $P_{2}(\cos \theta)$ exhibit similar oscillations, indicating peaks of mass density at positions where the chains are parallel to the interface [39]. 
a.

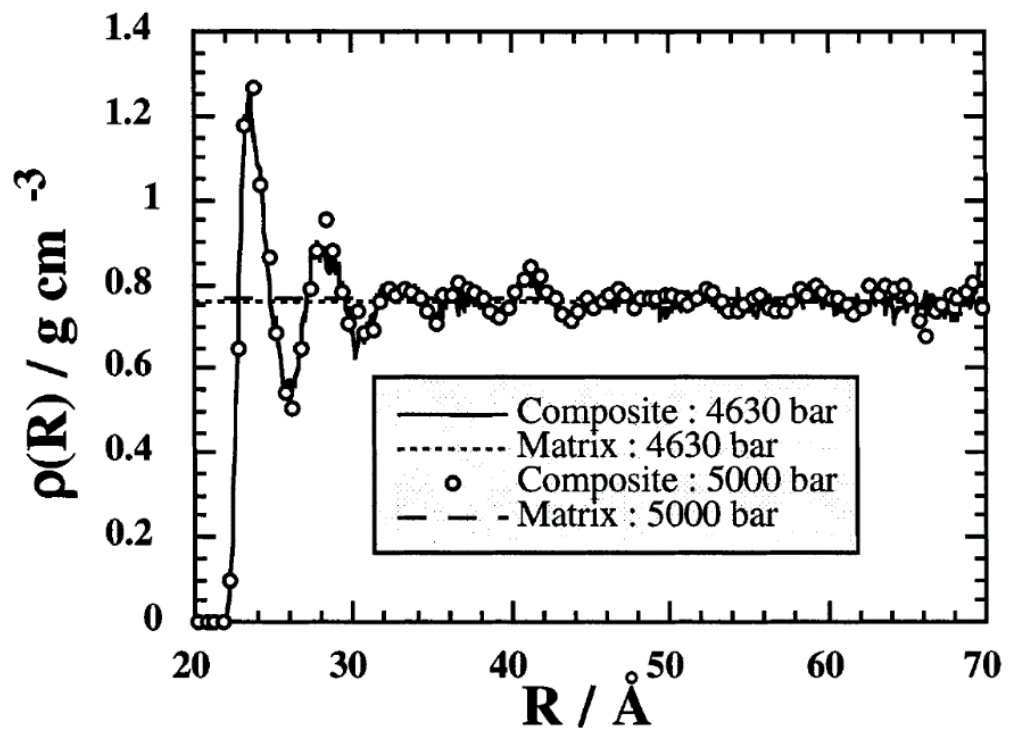

b.

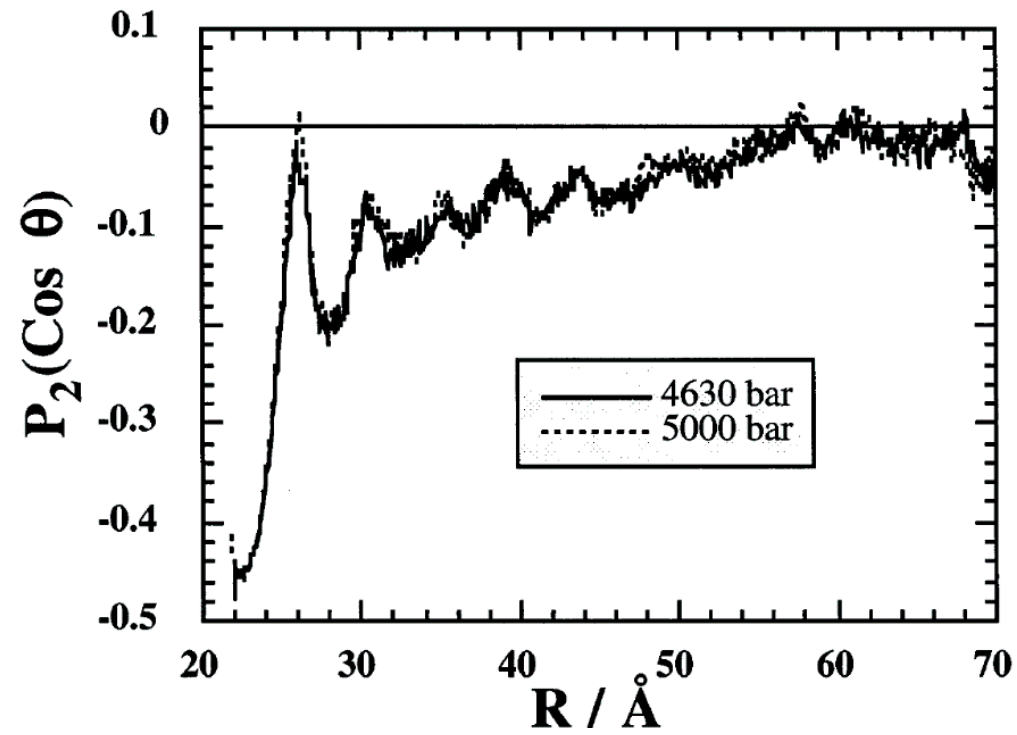

Figure 2.3. a: Mass density of the polymer as a function of the distance from the center-of-mass of the silica particle. The dotted and dashed lines are determined from the pure polymer simulations at the denoted pressures. b: Mean values of $P_{2}(\cos \theta)$ as a function of $\mathrm{R}$ for the composite system at the two pressures. The angle $\theta$ is defined as that between the outward vector normal to the interface and the local axis of the polymer chain ${ }^{40}$. 
Moreover, an increased number of chain ends is expected close to the NP surface because the decrease in entropy is expected to be lower if one end of the polymer chain is in contact with the NP ${ }^{39}$. These studies reveal the peculiar characteristics of the interfacial polymer chains compared to the bulk material. This notion is the founding assumption behind the interfacial models in nanodielectrics.

\subsection{The Interface Models}

As discussed above, the properties of polymer chains in the close vicinity of the NPs are considerably different than those of the bulk material. Due to the large specific surface area of NPs, these interfacial regions are in fact very significant, and are expected to greatly affect the macroscopic properties of the material, including the dielectric properties. Accordingly, the physical properties of the interfacial material close to the particle surface, is expected to be similar to those of the particle, and the further the distance from the particle, the closer the properties to those of the polymer ${ }^{16}$. The variation of the properties with respect to the distance from the particle is then dependent on the property being studied.

Presently, a model for describing interfaces in polymer nanodielectrics has been developed, for which two different formulations have been proposed ${ }^{41-42}$.

\subsubsection{Tanaka Model}

This model is based on the assumption that the interfacial zone around NPs generally consists of three layers, with an overall thickness of several tens of $\mathrm{nm}$, comparable to the NP diameter, and a fourth electric double layer which induces long distance interactions with other species. The latter is generated due to the charge transfer between the particle and polymer chains ${ }^{43}$. This multi-layer core model is shown in Figure 2.4 , and consists of the following layers. 


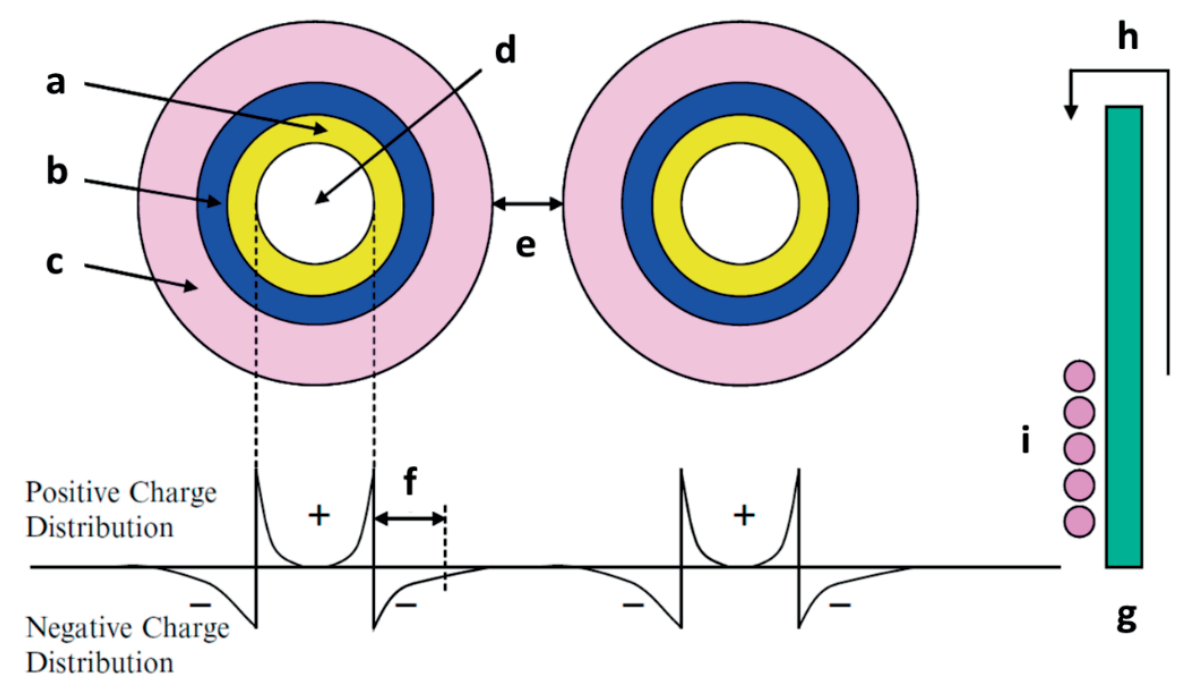

Figure 2.4. Illustration of the multi-layer core model; a: Bonded layer; b: Bound layer which in this model is considered to contain deep traps; c: Loose layer characterized by a large free volume (low density) containing shallow conformational traps: several tens of $\mathrm{nm}$ thick; $\mathrm{d}$ : nanoparticle; e: interparticle distance; $f$ : Debye shielding distance: a damping of the electric field due to the presence of mobile charge carriers; g: electrode exposed to the charging effect of nanoparticles; h: charge carrier injection by Schottky emission at high electric fields; i: charge tail effect of nanoparticles affecting carrier injection from electrode ${ }^{41}$

\subsubsection{Bonded Layer}

The bonded layer is related to the transition layer directly bonded to the surface of the NPs. Any surface group present directly on the NPs' surface or modifying agents used to treat the NPs are considered in this layer. Also in some cases, the bonded layer includes the polymer chains that are covalently bonded to the NPs ${ }^{44-45}$. However, the latter is not always the case, particularly in case of non-conjugated polymers such as polypropylene.

\subsubsection{Bound Layer}

The bound layer is generally the interfacial region where polymer chains are strongly bound to the first layer, with a rather orderly morphological structure. The thickness of this layer is reported to be in the range of $2-9 \mathrm{~nm}^{41}$, and is strongly dependent on the type of interactions between the filler and the polymer chains. Crystallinity and morphological properties of the nanodielectrics are immensely influenced by the interaction in the bound layer. 


\subsubsection{Loose Layer}

The third layer consists of a region where the chains are loosely bound to the second layer. The loose layer generally exhibits different chain conformation, mobility, free volume or crystallinity than the bulk polymer matrix ${ }^{46}$. The high free volume and low density of this layer may induce conformational traps, mostly in the shallower energy levels ${ }^{47}$.

\subsubsection{Electric Double Layer}

In addition to the layers introduced above, Tanaka argues that a charged region as a result of a Coulombic interaction is superimposed ${ }^{41}$. Due to the differences between the Fermi levels of the particle and polymer, the nanoparticle surface has a surface charge: a 2D interface called the Stern layer ${ }^{48}$ (will be discussed later). Accordingly, charge may be injected from the NP to the polymer matrix depending on their electron affinity, their Fermi levels or electrochemical potential. If charge injection occurs, the distribution of charge would form a Gouy-Chapman diffuse layer, similar to the injection from the metallic electrode into the dielectric, explained in the beginning of this chapter ${ }^{42}$. Since this region contains mobile charges, it has a significant effect on the dielectric and conductive properties of a nanodielectric. Any alteration of the charge mobility, or charge distribution on the surface of the NP will influence the properties of this layer. The diffuse layer overlaps the other three layers in the multi-core model, and is screened by a charged layer in the polymer phase. Triboelectricity of different types of materials reveals that polyolefins such as polyethylene (PE) and polypropylene (PP) tend to attract electrons and become negatively charged, whereas polymers like silicon rubber, polyamide and epoxy resin are expected to be positively charged ${ }^{49-51}$. This is shown in Figure 2.4 as the positive and negative charge distribution around the NP and in the interface.

\subsubsection{Lewis Model}

This formulation was developed based on the electrochemical interactions between a metallic electrode and a liquid electrolyte ${ }^{42}$. In his model, Lewis uses similar principles to that of a charged particle in an electrolyte. A surface charge layer, arising from the surface states of the particle, immobile charged impurities, trapped charges and mobile electrons and holes, forms the first layer in Lewis's formulation. Assuming a particle with positive 
surface charge, this situation would result in the polarization of the host material, the extent of which depends on the permittivity of the matrix. Also, this surface charge can be screened by ionic charge carriers in the polymer matrix. This may result in the formation of a second layer, i.e. Stern layer, adjacent to the NP surface (see Figure 2.5). The electrochemical potential will then drive counter-ions towards the NP surface and the co-ions away from it, resulting in the formation of a third layer: a diffuse layer. While the charges in the Stern layer are unlikely to be drifting under the electric field, the diffuse layer can contribute to conduction processes ${ }^{52}$, and has a significant effect on the overall dielectric behavior of the nanodielectric.

Lewis also mentions in his model the effect of active interfaces. He argues that the charged layer on the NP can constitute deep trap states, which together with the charged diffuse layer in the interface, can form a stable distribution of space charge or MaxwellWagner polarizations ${ }^{53}$. Furthermore, the concentration of charges in the diffuse layer is highly dependent on the induced potential between the particle and polymer ${ }^{48}$. These charges are mostly mobile counter-ions (opposite to the surface charge of the particle), and can largely participate in conduction processes. This represents an active interface, where the system undergoes major reorganization under the applied electric field. One important implication of this argument, is that space charge formation and conduction processes can be effectively controlled by altering the electronic structure of the polymer as well as the particle surface. The latter is possible e.g. by surface functionalization and modification of the NPs.

Based on the principles of the interface model, it can be postulated that apart from the structural and morphological aspects of the interface, the dielectric response of a nanodielectric is also affected by the electronic structure and surface states of the filler. Accordingly, tailoring these surface states can induce significant alterations in charge carrier mobility, space charge formation and conduction processes under electric fields. Another implication of the current model is that space charge accumulation near the electrodes, i.e. homocharge and heterocharge, depends largely on the injection rate, mobility and concentration of charge carriers at the interfaces located near the electrodes. 


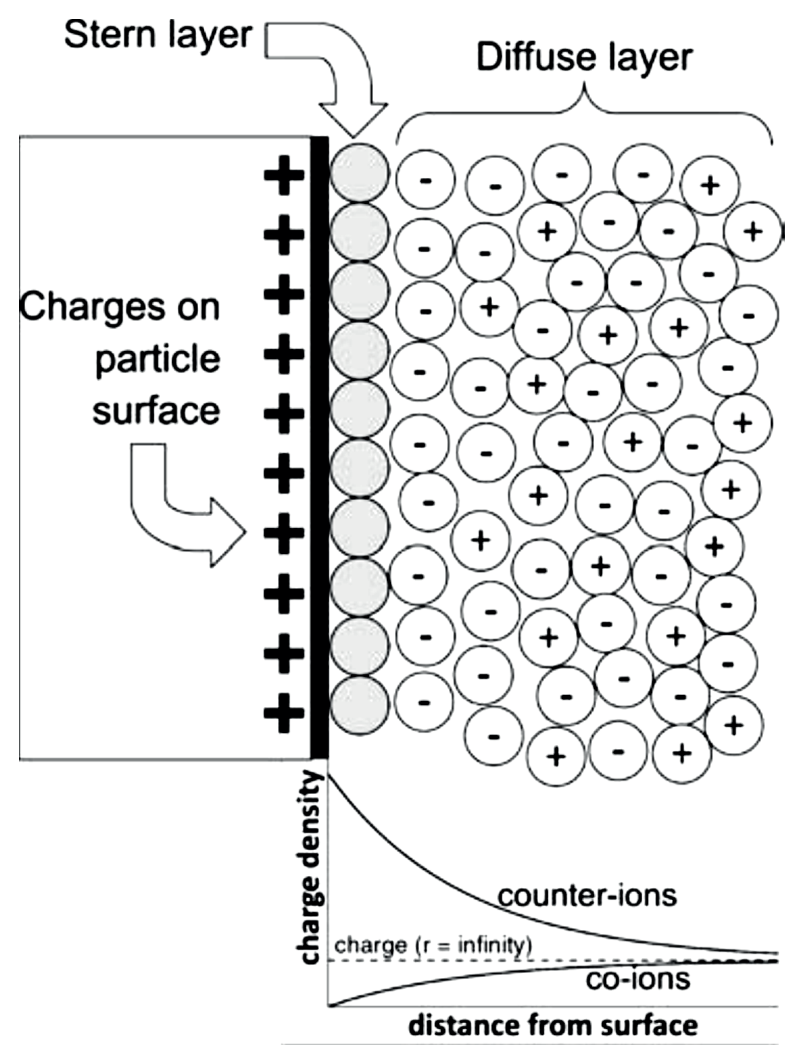

Figure 2.5. The diffuse electrical double layer induced by a charged particle in a polymer matrix containing mobile ions ${ }^{16}$.

In the following section, selected papers are discussed to cover different combinations of polymer/particle systems reported in the literature. The objective is to present comprehensive evidence to qualitatively support the principles of the interface model and the mechanistic aspect of nanodielectric phenomena.

\section{Historic Developments in Nanodielectric Materials}

One of the earliest studies of dielectric nanocomposites after the development of the interface model by Lewis and Tanaka, was the work of Nelson and Fothergill ${ }^{45}$. In this study, nanocomposites based on epoxy resin as matrix and titanium dioxide $\left(\mathrm{TiO}_{2}\right) \mathrm{NPs}$ were subjected to dielectric measurements. 

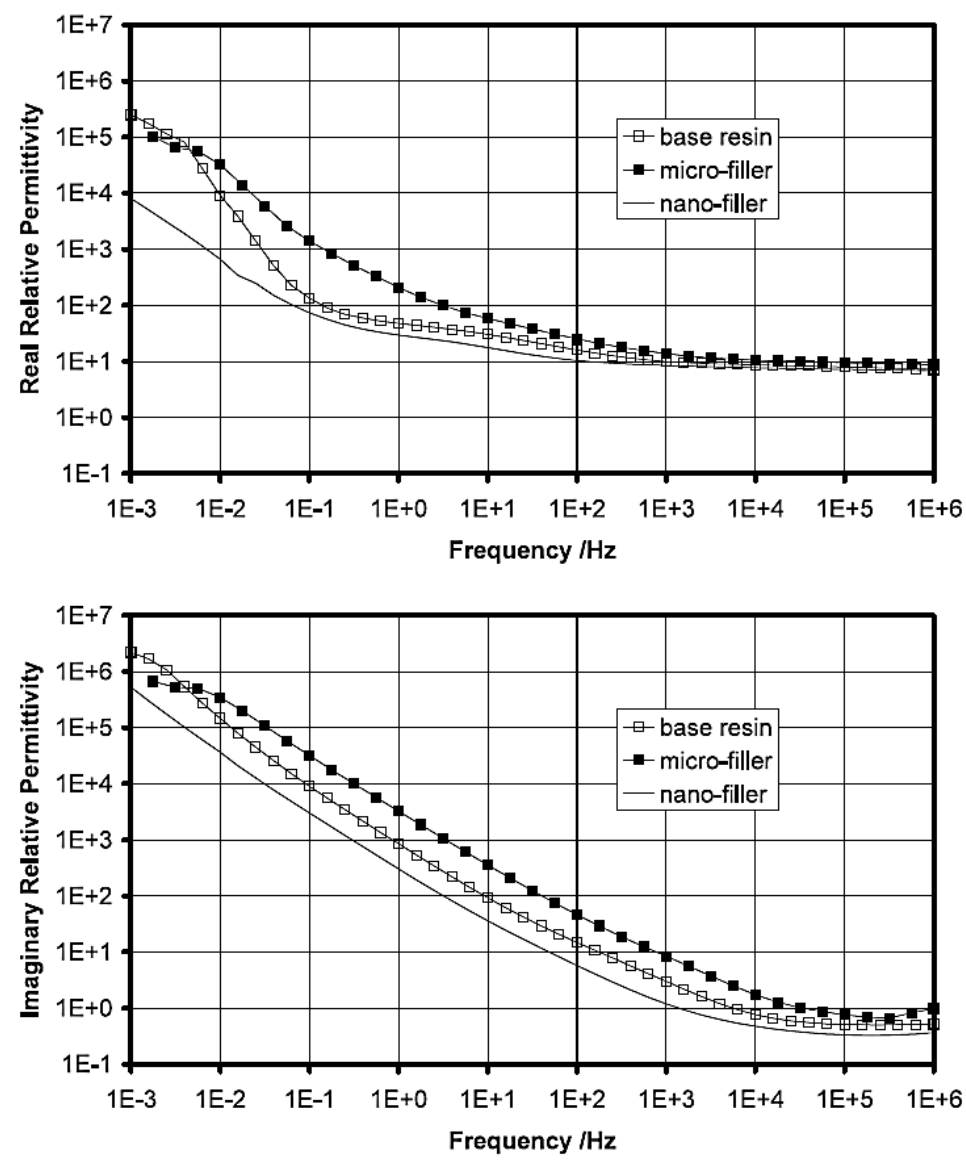

Figure 2.6. Real and imaginary relative permittivity of the unfilled polymer (base resin), compared to those of the micro- and nanocomposite samples at $393 \mathrm{~K}{ }^{45}$.

By comparing the results with a microcomposite reference, they observed a suppression in the real and imaginary permittivity of the nanocomposite, particularly in the lower range of frequencies (see Figure 2.6). In general, variations in permittivity with respect to the frequency of the field is determined by the dipolar molecular relaxations of the polymer chains, filler or any other species in the system, as well as the interfacial polarizations (related to space charge relaxations), depending on the frequency range. The dipolar relaxations are mainly predominant at higher frequencies, whereas the interfacial polarizations can be characterized as low frequency phenomena (usually below $1 \mathrm{~Hz})^{19}$. 
They concluded that the incorporation of NPs has substantial effects in reducing MaxwellWagner interfacial polarizations related to the formation of space charge.

Roy et al. ${ }^{44}$ focused on micro- and nanocomposites of crosslinked polyethylene (XLPE) and silica. In some cases, the NPs were functionalized by three types of silane modifying agents: triethoxyvinylsilane (TES), aminoethyl-aminopropyl-trimethoxysilane (AEAPS), or hexamethyldisilazane (HMDS). The first silane can also form covalent bonds with the polymer chains, resulting in a strong polymer/filler interaction, while AEAPS and HMDS are rather covering agents only bonding with the filler. Thermally stimulated depolarization current (TSDC) is a useful technique to quantitatively analyze the charge trapping and transport phenomena in nanodielectrics, by monitoring the discharge current when a charged sample is thermally stimulated ${ }^{54}$. This would result in a spectrum that is able to reveal all low-frequency motions in the material. The discharge current is, in fact, related to the release of charges accumulated at the interfaces. Therefore, peaks appearing above the glass transition temperature of the polymer can, in general, be attributed to the space charge relaxations. Accordingly, the temperature and the corresponding current of the peak can be correlated to the depth and density of charge traps in the material. Figure 2.7 shows the TSDC spectra for all the materials studied by Roy et al. Both the microcomposite and the untreated nanocomposite exhibit a peak at $\sim 54{ }^{\circ} \mathrm{C}$ which is attributed to the depolarizations regarding amorphous-crystalline interfaces of the polymer ${ }^{55}$. This peak is slightly shifted to lower temperatures for the treated samples. At higher temperatures, a significant rise is observed in the discharge current of the microcomposite, which is suppressed in case of the untreated and TES modified nanocomposites. This is indicative of the reduced space charge relaxations when incorporating NPs into the polymer. Grafting of the polymer to the filler does not have a significant effect in this case. AEAPS and HMDS modified samples show a rather different behavior. The space charge peaks at $91^{\circ} \mathrm{C}$ can be attributed to the introduction of deep traps at the interfaces. This is likely due to the change in the surface charge of the NPs induced by the grafted functional groups. 


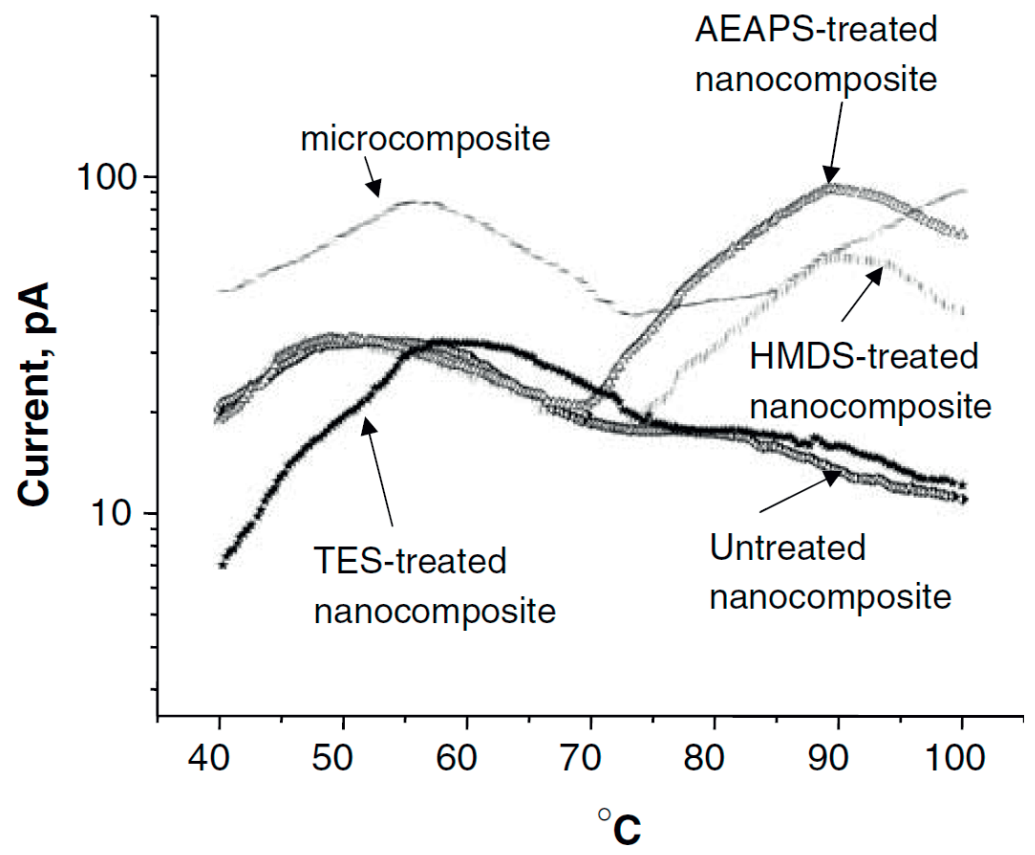

Figure 2.7. Thermally stimulated depolarization current spectra for XLPE/silica composites ${ }^{44}$.

Modification of NPs with polar functional groups was studied by Dongling et al ${ }^{56}$. They reported a higher dielectric breakdown strength and improved space charge distribution by introduction of amino functional groups to the surface of $\mathrm{TiO}_{2}$ nanoparticles incorporated in an LDPE matrix. They attributed this effect to the reduced acceleration of the electrons in the presence of polar amino groups, which made the dielectric less susceptible to breakdown ${ }^{57}$. Siddabattuni et al. ${ }^{58}$ investigated epoxy nanocomposites with $\mathrm{TiO}_{2}$ and $\mathrm{BaTiO}_{3} \mathrm{NPs}$, and observed that upon introduction of electron-withdrawing phenyl groups at the polymer/particle interface, the leakage current and dielectric loss was significantly reduced. This also led to an improvement of dielectric breakdown strength.

As mentioned above, one general explanation for this kind of effect from polar functional groups on NPs can be the alterations of the electronic structure of the filler surface. In principle, it is possible to determine the electronic structure of a material using quantum mechanical calculations; more particularly, density functional theory (DFT) ${ }^{33}$. This will be discussed in the next section. 


\section{Density Functional Theory (DFT)}

The principles of density functional theory (DFT) are rooted in the conventional wave function theory, where the electronic structure of matter is realized by the solvation of the Schrödinger equation ${ }^{33}$. The Schrödinger equation is, in fact, the quantum counter-part of the second law of Newton in classical mechanics. The same way as Newton's second law makes mathematical predictions as to what path a physical system takes over a period of time, Schrödinger's equation gives the evolution of a wave function, the quantum mechanical characteristic of a physical system ${ }^{59}$. It is generally recognized that a great deal of properties of matter are governed by its electronic structure, which in principle, can be realized by calculating the system's many-nuclei, many electron wave function ${ }^{60-61}$. Nevertheless, due to the fact that the wave function of a many-body system is a complicated object consisting of a large amount of information, exact analytical calculations become almost impossible ${ }^{62}$. DFT provides a way to bypass this computational difficulty, by focusing on the electron density rather than the many-body wave functions ${ }^{63}$. This is done firstly by using the Born-Oppenheimer approximation ${ }^{64}$, through which the electronic and nuclear degrees of freedom are separated. The justification for this is that the electrons are much less massive than the nuclei but experience similar forces, and therefore, the electrons will respond almost instantaneously to the movement of the nuclei. Thus, the energy for a given nuclear configuration will be that of the ground state of the electrons in that configuration. Accordingly, given a chemical structure with a certain set of elements, DFT succeeds in predicting the thermodynamically stable, ground state structure of the material, under the given conditions such as pressure, temperature, electric field etc. DFT methods do not rely on any experimental input, which is why they are often referred to as first principle or $a b$ initio methods ${ }^{65}$. In most cases, the time-dependent interactions of nuclei and electrons are neglected, and focus would be on the time-independent Schrödinger's equation. For an isolated n-electron atomic or molecular system, and considering the Born-Oppenheimer approximation, we can write the time-independent Schrödinger equation as

$$
\widehat{H} \Psi=E \Psi
$$

(Equation 2-5) 
where $\mathrm{E}$ is the electronic energy, $\Psi=\Psi\left(x_{1}, x_{2}, \ldots, x_{n}\right)$ is the wave function, and $\widehat{H}$ is the Hamiltonian operator,

$$
\widehat{H}=\sum_{i=1}^{n}\left(-\frac{1}{2} \nabla_{i}^{2}\right)+\sum_{i=1}^{n} v\left(r_{i}\right)+\sum_{i<j}^{n} \frac{1}{r_{i j}}
$$

in which,

$$
v\left(r_{i}\right)=-\sum_{\alpha} \frac{Z_{\alpha}}{r_{i \alpha}}
$$

is the potential acting on electron $i$, from the nuclei $Z_{\alpha} \cdot x_{i}$ are coordinates of electron $i$, and consist of its space coordinates $r_{i}$ and spin coordinates $s_{i} \cdot \nabla_{i}^{2}=\frac{\partial^{2}}{\partial x_{i}^{2}}+\frac{\partial^{2}}{\partial y_{i}^{2}}+\frac{\partial^{2}}{\partial z_{i}^{2}}$ is the Laplacian operator. The length unit is considered the Bohr radius ( $a_{0}=0.5292 \AA$ ), the charge unit is the charge of an electron $e$, and the mass unit is the mass of the electron $m_{e}$. Equation 2-6 can be written more compactly as

$$
\widehat{H}=\widehat{T}+\widehat{V}_{n e}+\widehat{V}_{e e}
$$

where

$$
\widehat{T}=\sum_{i=1}^{n}\left(-\frac{1}{2} \nabla_{i}^{2}\right)
$$

is the kinetic energy operator,

$$
\widehat{V}_{n e}=\sum_{i=1}^{n} v\left(r_{i}\right)
$$

is the electron-nucleus attraction energy operator, and

$$
\widehat{V}_{e e}=\sum_{i<j}^{n} \frac{1}{r_{i j}}
$$


is the electron-electron repulsion energy operator. The total energy of the system, W, is then the sum of electronic energy $E$ (from Equation 2-5) and $\widehat{V}_{n n}$ the nucleus-nucleus repulsion energy.

$$
\begin{gathered}
W=E+\hat{V}_{n n} \\
\hat{V}_{n n}=\sum_{\alpha<\beta} \frac{Z_{\alpha} Z_{\alpha \beta}}{R_{\alpha \beta}}
\end{gathered}
$$

One can first solve Equation 2-5 to get $\mathrm{E}$, and add $\widehat{V}_{n n}$ afterwards, or include $\widehat{V}_{n n}$ in the definition of $\widehat{H}$ and work with the Schrödinger equation in the form of $\widehat{H} \Psi=W \Psi{ }^{33}$.

Let us denote the ground state wave function and energy of the system by $\Psi_{0}$ and $\mathrm{E}_{0}$. When a system is in the state $\Psi$, whether or not it satisfies Equation 2-5, the average of many measurements of energy can be written as

$$
E[\Psi]=\frac{\langle\Psi|\widehat{H}| \Psi\rangle}{\langle\Psi \mid \Psi\rangle}
$$

$\langle\Psi \mid \Psi\rangle$ is a superposition of all possible wave functions, and $\langle\Psi|\widehat{H}| \Psi\rangle$ is the Hamiltonian operator acting on that superposition. Since each measurement of the energy will give one of the eigenvalues of $\widehat{H}$, it immediately leads to

$$
E[\Psi] \geq \mathrm{E}_{0}
$$

(Equation 2-15)

The computed energy from a guessed wave function $\Psi$ would be higher than the ground state energy $\mathrm{E}_{0}$. Therefore, minimization of the energy functional $E[\Psi]$ with respect to all allowed wave functions will give the ground state $\Psi_{0}$ and $E_{0}$ as

$$
\mathrm{E}_{0}=\min _{\Psi} E[\Psi]
$$

The electrons of the system occupy the valence energy states with the last electrons at the highest occupied molecular orbital (HOMO). DFT also reveals the energy levels of unoccupied orbitals, of which the lowest unoccupied molecular orbital (LUMO) is often taken into consideration. 
The first step towards this computational approach is the construction of an appropriate atomic or molecular model of interest. This can be done by means of several software, e.g. GaussView which allows to define the chemical structure and the computational details to produce an input file for the calculation. This input file containing all required information (atoms, their coordinates, and the adopted computational methods) is then imported into another software (e.g. Gaussian ${ }^{66}$ ) to perform the calculations. The atomic coordinates are then optimized by the minimization of the total energy of the system $E[\Psi]$. The calculated electronic energies also provide an estimate of the band gap, and when available, the energy levels of localized states or traps.

As mentioned above, DFT uses simplifying assumptions to make the treatment of the many-body problem possible. Accordingly, it seems fair to question the accuracy of this method in describing the properties of the system. The strongest aspect of DFT is its ability to predict structural details of the model, typically within $1 \%$ error with respect to experimental data ${ }^{67-69}$. DFT also predicts dielectric constants for insulators typically within $5 \%$ of actual measurements ${ }^{70-72}$. The most important drawback of DFT lies in its inability to predict band gaps of insulators and semiconductors, with the same accuracy as for other properties ${ }^{16}$. DFT usually underestimates the band gaps with respect to experimental data, sometimes by $50 \%{ }^{73}$. Nevertheless, some techniques are available to handle this issue, which result in a satisfactory correlation ${ }^{73-76}$. Therefore, with the currently available techniques, DFT is capable of predicting fundamental properties of the model with acceptable accuracy.

\subsection{Application of DFT for Nanodielectrics}

The study of the properties of interfaces in nanodielectrics, in particular, the electronic structure at the interface, is possible using DFT methods. The electronic structure would contain information, for instance, about the location of valence and conduction band extrema and localized states (traps) which control the conduction processes and space charge formation phenomena. Furthermore, DFT allows for calculating the density of states (DOS) to provide quantitative information about the number of allowed energy levels within a given range ${ }^{77-78}$. Figure 2.8 presents the density of states (DOS) calculated for a silica/PVDF 
interface, where the filler and polymer are covalently bonded with a vinylsilanediol coupling agent ${ }^{77}$. It can be observed that the DOS in the middle parts of silica and PVDF (marked with red lines) exhibit large uniform band gaps. However, in the region containing the coupling agent, the band extrema do not evolve smoothly. Two peaks appear (marked with circles): one at a lower energy level corresponding to an occupied defect state, and the other related to an unoccupied localized state. These peaks, located at the edges of the valence and conduction bands, demonstrate the presence of localized states for holes and electrons, respectively. These traps can potentially immobilize conducting electrons and holes, and reduce conduction processes and space charge formation in the nanodielectric.

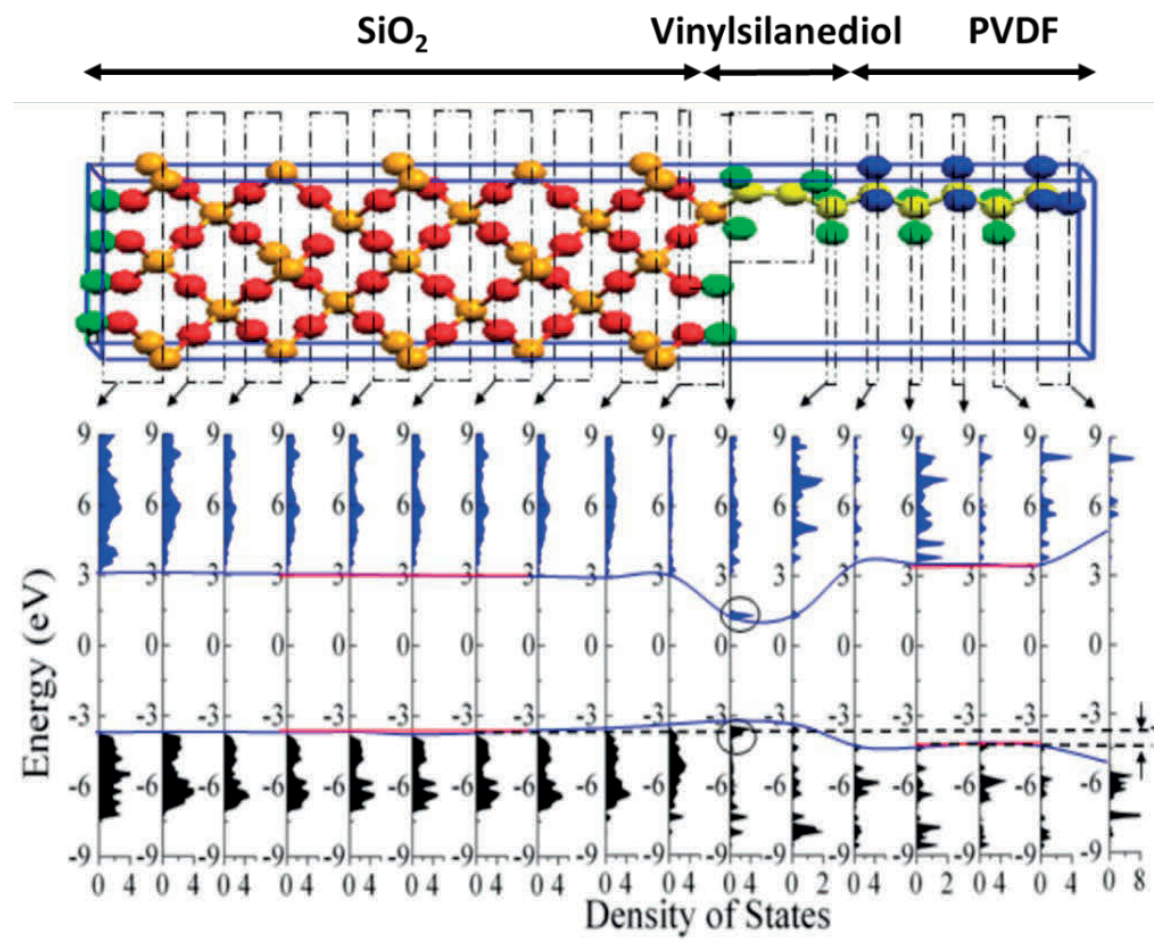

Figure 2.8. Above: Atomic model of $\mathrm{SiO}_{2}$ /vinlysilanediol/PVDF interface; (Si: gold, C: yellow, O: red, $\mathrm{H}$ : green and $\mathrm{F}$ : blue). Below: Density of States for the $\mathrm{SiO}_{2} /$ vinlysilanediol/PVDF interface (conduction band: blue, valence band: black). The zero of energy is related to the Fermi level. Lower and upper circled states represent occupied and unoccupied interfacial localized states, respectively ${ }^{77}$. 
Despite the important information DFT can give us regarding the electronic structure of the interface and its effect on the dielectric response of the material, little has been done in this area with respect to different combinations of filler, polymer and modifying agents. This approach can be utilized to find a link between the electronic structure of the interface, type of functional groups present at the interface and their ultimate influence on charge trapping and transport phenomena in nanodielectrics. Nevertheless, since these relations are highly dependent on the set of materials being investigated, more studies are required to develop our understanding of the peculiar phenomena taking place at the interfaces.

\section{Surface Modification of Nanoparticles}

In the previous sections, we mentioned the possibility to modify the surface chemistry of nanoparticles for tailoring their interfacial interactions with the polymeric matrix. In general, applications of nanoparticles in composite materials are based on their properties e.g. porosity, active surface area, hardness and rheological characteristics. Other specific requirements regarding the role of nanoparticles can be achieved through modification of their surface chemical structure. Surface modification can be defined as the "chemical bonding of molecules or molecule fragments to a surface in order to change its chemical or physical properties in a controlled way" 79 . A nanoparticle requires seemingly paradoxical properties in order to be modified effectively: i) it needs to have a hydrophilic surface, yet remain insoluble in aqueous solutions; ii) it is required, in many cases, to be porous but remain mechanically stable; and iii) it must be chemically stable yet easily reactive in certain conditions ${ }^{80}$. Silica satisfies all these requirements. Despite the disadvantages such as moisture absorption, silica is a well understood material, and therefore, a proper choice to be used for proof of concept.

Organofunctional silanes tend to be among the best choices of materials to be used as the modifying agent for silica. Organofunctional silanes can potentially bond to the silica surface through several mechanisms, and have different types of interactions with the silica surface e.g. van der Waals forces or hydrogen bonding ${ }^{81}$. 
Modification of nanoparticles is conventionally performed in the liquid phase. Both aqueous (water, water/ethanol or water/acetone) and organic non-aqueous (e.g. toluene, hexane etc.) solvents can be used as the reaction medium ${ }^{82-83}$. In general, liquid-phase modification methods often lack a systematic control over the morphology and thickness of the deposited layer. In the aqueous solvents, the silanes can undergo hydrolysis and condensation before deposition on the surface, and hence grow almost uncontrollably. The non-aqueous approach can offer limited control over the hydrolysis and the deposited film morphology by carefully adjusting the amount of water in the reaction. Nevertheless, the use of hazardous organic solvents can be challenging for large scale industrial modification of nanoparticles. Furthermore, liquid-phase modification methods often suffer from issues such as solvent recovery, long operation times, high costs and low efficiency ${ }^{84}$.

This is where atomic layer deposition (ALD) and its organic counterpart, molecular layer deposition (MLD) techniques enter the scene. ALD and MLD are gas-phase deposition methods that, by relying on gas-solid surface reactions, can potentially address the challenges that accompany conventional chemistry techniques, particularly regarding controllability and scalability ${ }^{85}$. Both methods offer outstanding control over the amount of deposited material, down to the atomic scale, by relying on self-limiting surface reactions. The desired compound is synthesized on the substrate surface by the sequential dosing of two or more precursors into the reactor, with purging steps in between. The purge step, generally done by pulsing an inert gas into the reactor, is crucial to remove reaction byproducts or unreacted precursor molecules after each step, in order to maintain the selflimiting fashion of the process. Repeating these steps in a cyclic manner, would result in the growth of the desired material on the surface with nanoscale precision ${ }^{86}$. Theoretically, each cycle should result in the growth of a monolayer of the deposited material. In reality, however, a sub-monolayer is deposited per cycle due to a number of limitations such as steric hindrance of the precursor molecules, the surface density of the functional groups and competitive adsorption/desorption phenomena on the surface ${ }^{87}$. Fluidized beds are the most effective type of the reactors utilized for functionalization of nanoparticles with ALD and MLD ${ }^{88-89}$. In this case, the particles are suspended ('fluidized') in a column reactor by means of an inert gas flow (typically nitrogen), passing through a distributor plate. During 
the process, the nitrogen flow will also carry the precursors into the reactor while keeping the powder fluidized. Figure 2.9 shows a schematic illustration of this setup.

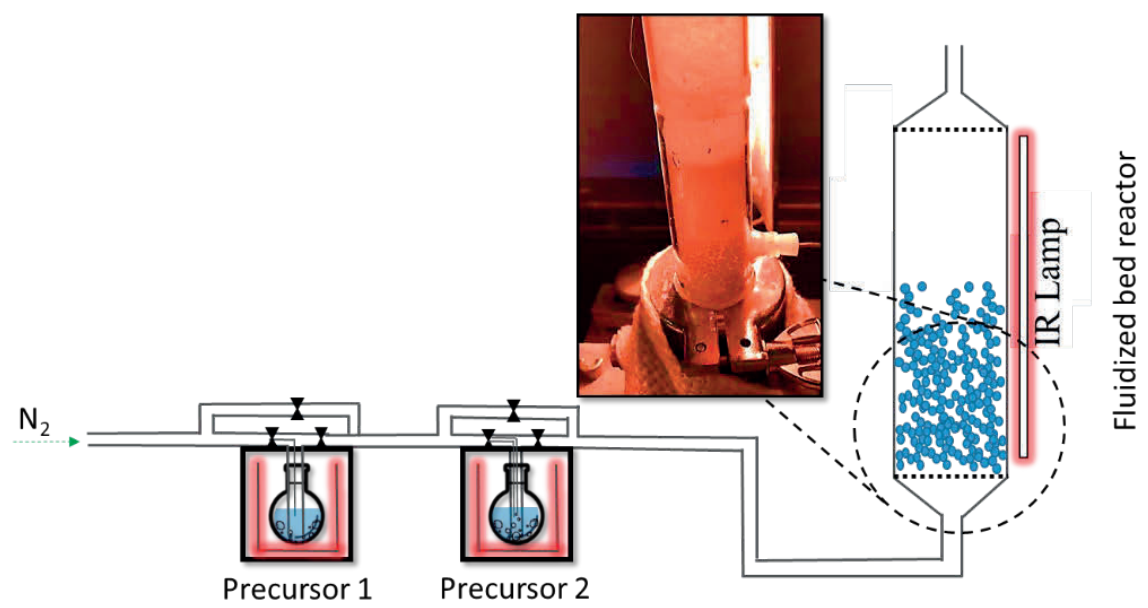

Figure 2.9. Schematic of the ALD/MLD setup with a fluidized bed reactor

Using ALD and MLD methods, it is possible to deposit a wealth of materials on different surfaces. Metal oxides such as $\mathrm{AL}_{2} \mathrm{O}_{3}, \mathrm{TiO}_{2}, \mathrm{CuO}$ and $\mathrm{HfO}_{2}$ are among the most common materials that can be deposited via ALD, which find applications e.g. in the semiconductor technologies ${ }^{90-91}$. MLD uses the same principles as ALD and deposits organic or hybrid organic/inorganic films. Polymeric thin films with several applications in optics, electroluminescent and photochromic materials can be grown on surfaces via MLD ${ }^{92}$. Adamczyk et al. were the first to perform MLD on nanoparticles instead of flat substrates 93. They reported successful deposition of poly ( $p$-phenylene terephthalamide) (PPTA) thin films on silica nanoparticles. Liang et al. used fluidized bed MLD to grow alucone films on silica and titania nanoparticles ${ }^{94}$. MLD has also been utilized to deposit conformal layers of polyurea from ethylene diamine (ED) and phenyl diisocyanate (PDIC) onto carbon nanotubes (CNTs) ${ }^{95}$.

Given the versatility and scalability of these methods, and their significant advantages over the conventional solvent-based methods, ALD and MLD can be promising tools to functionalize nanoparticles also for nanodielectric applications. Accordingly, ALD and MLD are great tools to tailor the surface chemistry of nanoparticles, and consequently, alter the 
interfacial properties of the nanocomposites. By depositing proper compounds with certain functional groups, significant improvements can be bestowed upon the dielectric response of the material. This is a major part of the present thesis, and will be discussed in Chapters 6 to 9 .

\section{The Polymer of Choice}

As mentioned in the previous chapter, the appealing promise of polymeric nanodielectrics has attracted high voltage cable industries to incorporate these materials into their production, which is also the final goal of this project. The choice of the appropriate polymeric matrix, therefore, is of both technical and practical importance for large scale production of extruded cables: the polymer of choice has to exhibit superior dielectric performance, and it has to be abundantly produced by material suppliers. Low density polyethylene (LDPE) has been used as the insulation system for high voltage alternating current (HVAC) cables for about five decades, due to its excellent electrical properties, flexibility, and low processing costs ${ }^{96-97}$. However, LDPE is not a suitable choice for large capacity high voltage direct current (HVDC) cables, since its operating temperature is limited to $70{ }^{\circ} \mathrm{C}^{98}$. Using radical reactions with organic peroxides e.g. dicumyl peroxide (DCP), LDPE can be turned into crosslinked polyethylene (XLPE) to achieve higher operational temperatures of about $90{ }^{\circ} \mathrm{C}^{99}$. Owing to its promising dielectric performance, high operating voltage and temperature and low dielectric loss, XLPE has been the major choice for extruded HVDC cable insulation systems, and is being used in the existing grids 100-101. The downside to this material is that the crosslinking byproducts such as phenol, cumyl alcohol, acetophenone and $\alpha$-methyl styrene may diminish the electrical properties and lead to space charge accumulation ${ }^{102}$. Furthermore, due to the thermosetting nature of this polymer, XLPE cables cannot be recycled using conventional methods ${ }^{103-104}$. As a result, special attention is paid to thermoplastic and thus recyclable polymers such as polypropylene (PP) and ethylene-propylene rubber (EPM). EPM, on the one hand, can exhibit superior properties compared to XLPE, however, it is generally expensive and its application in large scales would be limited ${ }^{105}$. PP, on the other hand, has shown immense potential to become the next generation of HVDC insulation material; it is cheap and 
abundantly produced, is easy to process, shows very good electrical properties, and last but not least, it is recyclable ${ }^{106-107}$. Despite its immense promise for a high performance and environmentally friendly HVDC cable insulation, PP suffers from poor mechanical properties. Several studies have been done focusing on improving the mechanical properties of PP, and it was realized that the most effective way to so is to blend it with thermoplastic elastomers such as ethylene-propylene-diene rubber (EPDM), styreneethylene-butylene-styrene (SEBS) and ethylene-octene copolymer (EOC) ${ }^{106,108-109}$. This way, it is possible to achieve a polymeric material to equip HVDC grids with long-lasting environmentally friendly cables. Green et. al ${ }^{110}$ reported improved electrical properties when using an isotactic PP/EPC blend as the polymeric matrix, and argued that the optimal properties occur when the copolymer contains $9 \mathrm{~mol} \%$ of ethylene and equal quantities of each component are incorporated into the blend. Zhou and coworkers ${ }^{106}$ studied the potential of PP/EOC blends for HVDC cable application. Their findings pointed out that these blends have an enhanced flexibility (compared to pure PP), high volume resistivity and high breakdown strength; nevertheless, they exhibited space charge accumulation. The issue of space charge accumulation in PP/EOC blends was later on addressed by Diao et. al ${ }^{111}$, by the introduction of silica nanoparticles. They envisaged that the space charge behavior in PP/EOC blends can be further improved by tailoring the surface properties of the nanoparticles via chemical modification. Given the wide range of possible modifications for the nanoparticle surface, a systematic study is necessary to further enhance the electrical properties of these blends. In this work, a PP/EOC blend is chosen as the polymeric matrix for the dielectric nanocomposites. To be able to solely investigate the effect of nanoparticle surface properties on the dielectric performance of the nanocomposites, this polymer of choice is kept constant in all investigations within this thesis. 


\section{References}

[1] Frechette, M. F.; Trudeau, M. L.; Alamdar, H.; Boily, S., Introductory remarks on nanodielectrics. IEEE Transactions on Dielectrics and Electrical Insulation, 11 (5), 2004, pp 808-818.

[2] Ray, S. S.; Okamoto, M., Polymer/layered silicate nanocomposites: a review from preparation to processing. Progress in Polymer Science, 28 (11), 2003, pp 1539-1641.

[3] Ajayan, P. M.; Schadler, L. S.; Braun, P. V., Nanocomposite science and technology John Wiley \& Sons: 2006;

[4] Deguchi, R.; Nishio, T.; Okada, A. High rigidity and impact resistance resin composition, Toyota Motor Corp., Ube Industries Ltd., Toyota Central R\&D Labs Inc., US5164440A, 1992.

[5] Koo, J. H., Polymer nanocomposites McGraw-Hill Professional Pub.: 2006;

[6] Kim, H.; Abdala, A. A.; Macosko, C. W., Graphene/polymer nanocomposites. Macromolecules, 43 (16), 2010, pp 6515-6530.

[7] Krawiec, W.; Scanlon Jr, L.; Fellner, J.; Vaia, R.; Vasudevan, S.; Giannelis, E., Polymer nanocomposites: a new strategy for synthesizing solid electrolytes for rechargeable lithium batteries. Journal of Power Sources, 54 (2), 1995, pp 310-315.

[8] Favier, V.; Chanzy, H.; Cavaillé, J., Polymer nanocomposites reinforced by cellulose whiskers. Macromolecules, 28 (18), 1995, pp 6365-6367.

[9] Schadler, L. S.; Nelson, J. K., Polymer nanodielectrics-Short history and future perspective. Journal of Applied Physics, 128 (12), 2020, p 120902.

[10] Lewis, T. J., Nanometric dielectrics. IEEE Transactions on Dielectrics and Electrical Insulation, 1 (5), 1994, pp 812-825.

[11] Helmholtz, H. V., Studien über electrische Grenzschichten. Annalen der Physik, 243 (7), 1879, pp 337-382.

[12] Chapman, D. L., LI. A contribution to the theory of electrocapillarity. The London, Edinburgh, and Dublin philosophical magazine and journal of science, 25 (148), 1913, pp 475-481.

[13] Gouy, M., Sur la constitution de la charge électrique à la surface d'un électrolyte. Journal of Physics: Theories and Applications, 9 (1), 1910, pp 457-468.

[14] Wintle, H., Charge motion and trapping in insulators: surface and bulk effects. IEEE Transactions on Dielectrics and Electrical Insulation, 6 (1), 1999, pp 1-10.

[15] McCubbin, W. Conduction processes in polymers, Journal of Polymer Science Part C: Polymer Symposia, Wiley Online Library: 1970; pp 181-193.

[16] Nelson, J. K., Dielectric polymer nanocomposites Springer: 2010;

[17] Wintle, H., Charge motion in technical insulators: facts, fancies and simulations. IEEE Transactions on Dielectrics and Electrical Insulation, 10 (5), 2003, pp 826-841.

[18] v. Schweidler, E. R., Studien über die Anomalien im Verhalten der Dielektrika. Annalen der Physik, 329 (14), 1907, pp 711-770.

[19] Jonscher, A. K., Dielectric relaxation in solids. Journal of Physics D: Applied Physics, 32 (14), 1999, p R57.

[20] Guo, T. C.; Guo, W. W., A transient-state theory of dielectric relaxation and the Curie-von Schweidler law. Journal of Physics C: Solid State Physics, 16 (10), 1983, p 1955. 
[21] Baird, M., Determination of dielectric behavior at low frequencies from measurements of anomalous charging and discharging currents. Reviews of Modern Physics, 40 (1), 1968, p 219.

[22] Lowell, J., Absorption and conduction currents in polymers: a unified model. Journal of Physics D: Applied Physics, 23 (2), 1990, p 205.

[23] Zaengl, W. S., Dielectric spectroscopy in time and frequency domain for HV power equipment. I. Theoretical considerations. IEEE Electrical Insulation Magazine, 19 (5), 2003, pp 5-19.

[24] Fleming, R.; Ammala, A.; Casey, P. S.; Lang, S., Conductivity and space charge in LDPE containing nano-and micro-sized ZnO particles. IEEE Transactions on Dielectrics and Electrical Insulation, 15 (1), 2008, pp 118-126.

[25] Fleming, R.; Pawlowski, T.; Ammala, A.; Casey, P. S.; Lawrence, K., Electrical conductivity and space charge in LDPE containing TiO/sub 2/nanoparticles. IEEE Transactions on Dielectrics and Electrical Insulation, 12 (4), 2005, pp 745-753.

[26] Dissado, L. A.; Fothergill, J. C., Electrical degradation and breakdown in polymers let: 1992; Vol. 9.

[27] Lewis, T. J., Electrical effects at interfaces and surfaces. IEEE transactions on electrical insulation, (3), 1986, pp 289-295.

[28] Ritsko, J.; Mort, J.; Pfister, G., Electronic states and triboelectricity. In Electronic Properties of Polymers, Wiley New York: 1982; pp 13-57.

[29] Androsch, R.; Di Lorenzo, M. L.; Schick, C.; Wunderlich, B., Mesophases in polyethylene, polypropylene, and poly(1-butene). Polymer, 51 (21), 2010, pp 4639-4662.

[30] Mazzanti, G.; Montanari, G.; Palmieri, F.; Alison, J., Apparent trap-controlled mobility evaluation in insulating polymers through depolarization characteristics derived by space charge measurements. Journal of Applied Physics, 94 (9), 2003, pp 5997-6004.

[31] Gross, B.; Sessler, G.; Von Seggern, H.; West, J., Hole transit in Teflon films. Applied Physics Letters, 34 (9), 1979, pp 555-557.

[32] Bambery, K.; Fleming, R., Space charge accumulation in two power cable grades of XLPE. IEEE Transactions on Dielectrics and Electrical Insulation, 5 (1), 1998, pp 103-109.

[33] Parr, R. G. Density Functional Theory of Atoms and Molecules, Horizons of Quantum Chemistry, Springer Netherlands: Dordrecht, 1980; pp 5-15.

[34] Fleming, R., Charge trapping in organic polymers. International Journal of Radiation Applications and Instrumentation. Part C. Radiation Physics and Chemistry, 36 (1), 1990, pp 59-68.

[35] Boyd, R. H.; Breitling, S., The conformational analysis of crankshaft motions in polyethylene. Macromolecules, 7 (6), 1974, pp 855-862.

[36] Lewis, T., Polyethylene under electrical stress. IEEE Transactions on Dielectrics and Electrical Insulation, 9 (5), 2002, pp 717-729.

[37] Redi, M.; Hopfield, J., Theory of thermal and photoassisted electron tunneling. The Journal of chemical physics, 72 (12), 1980, pp 6651-6660.

[38] Dutta, N.; Choudhury, N. R.; Haidar, B.; Vidal, A.; Donnet, J.; Delmotte, L.; Chezeau, J., High resolution solid-state NMR investigation of the filler-rubber interaction: 1 . High speed $1 \mathrm{H}$ magic-angle spinning NMR spectroscopy in carbon black filled styrene-butadiene rubber. Polymer, 35 (20), 1994, pp 4293-4299. 
[39] Liu, J.; Wu, Y.; Shen, J.; Gao, Y.; Zhang, L.; Cao, D., Polymer-nanoparticle interfacial behavior revisited: A molecular dynamics study. Physical Chemistry Chemical Physics, 13 (28), 2011, pp 13058-13069.

[40] Brown, D.; Mele, P.; Marceau, S.; Alberola, N., A molecular dynamics study of a model nanoparticle embedded in a polymer matrix. Macromolecules, 36 (4), 2003, pp 13951406.

[41] Tanaka, T.; Kozako, M.; Fuse, N.; Ohki, Y., Proposal of a multi-core model for polymer nanocomposite dielectrics. IEEE Transactions on Dielectrics and Electrical Insulation, 12 (4), 2005, pp 669-681.

[42] Lewis, T., Interfaces: nanometric dielectrics. Journal of Physics D: Applied Physics, 38 (2), 2005, p 202.

[43] Lewis, T. J., Nano-composite dielectrics: the dielectric nature of the nano-particle environment. IEEJ Transactions on Fundamentals and Materials, 126 (11), 2006, pp 10201030.

[44] Roy, M.; Nelson, J. K.; MacCrone, R. K.; Schadler, L. S., Candidate mechanisms controlling the electrical characteristics of silica/XLPE nanodielectrics. Journal of Materials Science, 42 (11), 2007, pp 3789-3799.

[45] Nelson, J. K.; Fothergill, J. C., Internal charge behaviour of nanocomposites. Nanotechnology, 15 (5), 2004, p 586.

[46] Nelson, J.; Utracki, L.; MacCrone, R.; Reed, C. Role of the interface in determining the dielectric properties of nanocomposites, The 17th Annual Meeting of the IEEE Lasers and Electro-Optics Society, 2004. LEOS 2004., IEEE: 2004; pp 314-317.

[47] Tanaka, T., Dielectric nanocomposites with insulating properties. IEEE Transactions on Dielectrics and Electrical Insulation, 12 (5), 2005, pp 914-928.

[48] Lewis, T. J., Interfaces are the dominant feature of dielectrics at the nanometric level. IEEE Transactions on Dielectrics and Electrical Insulation, 11 (5), 2004, pp 739-753.

[49] Saint Jean, M.; Hudlet, S.; Guthmann, C.; Berger, J., Local triboelectricity on oxide surfaces. The European Physical Journal B-Condensed Matter and Complex Systems, 12 (4), 1999, pp 471-477.

[50] Ueno, N.; Sugita, K.; Seki, K.; Inokuchi, H., Low-energy electron transmission and secondary-electron emission experiments on crystalline and molten long-chain alkanes. Physical Review B, 34 (9), 1986, p 6386.

[51] Bloor, D., Correlation of experimental and theoretical electron band energies of polyethylene. Chemical Physics Letters, 40 (2), 1976, pp 323-325.

[52] Grosse, C., Dielectric properties of suspensions of solid particles. Encyclopedia of surface and colloid science. Taylor and Francis, 2006, pp 1688-1705.

[53] Prodromakis, T.; Papavassiliou, C., Engineering the Maxwell-Wagner polarization effect. Applied Surface Science, 255 (15), 2009, pp 6989-6994.

[54] Turnhout, J. v., Thermally Stimulated Discharge of Polymer Electrets. Polymer Journal, 2 (2), 1971, pp 173-191.

[55] leda, M.; Mizutani, T.; Suzuoki, Y., TSC and TL studies of carrier trapping in insulating polymers. Memoirs of the Faculty of Engineering, Nagoya University, Nagoya, 32 (2), 1980, pp 173-219. 
[56] Ma, D.; Treese, A. H.; Siegel, R.; Anna, C.; Eva, M.; Carina, Ö.; Linda, S. S., Influence of nanoparticle surface modification on the electrical behaviour of polyethylene nanocomposites. Nanotechnology, 16 (6), 2005, p 724.

[57] leda, M., Dielectric breakdown process of polymers. IEEE transactions on electrical insulation, (3), 1980, pp 206-224.

[58] Siddabattuni, S.; Schuman, T. P.; Dogan, F., Dielectric Properties of PolymerParticle Nanocomposites Influenced by Electronic Nature of Filler Surfaces. ACS Applied Materials \& Interfaces, 5 (6), 2013, pp 1917-1927.

[59] Griffiths, D. J.; Schroeter, D. F., Introduction to quantum mechanics Cambridge University Press: 2018;

[60] Martin, R. M., Electronic structure: basic theory and practical methods Cambridge university press: 2020 ;

[61] Kohn, W.; Becke, A. D.; Parr, R. G., Density Functional Theory of Electronic Structure. The Journal of Physical Chemistry, 100 (31), 1996, pp 12974-12980.

[62] Dreizler, R. M.; Gross, E. K., Density functional theory: an approach to the quantum many-body problem Springer Science \& Business Media: 2012;

[63] Hasnip, P. J.; Refson, K.; Probert, M. I. J.; Yates, J. R.; Clark, S. J.; Pickard, C. J., Density functional theory in the solid state. Philosophical Transactions of the Royal Society A: Mathematical, Physical and Engineering Sciences, 372 (2011), 2014, p 20130270.

[64] Born, M.; Oppenheimer, R., Zur quantentheorie der molekeln. Annalen der Physik, 389 (20), 1927, pp 457-484.

[65] Hehre, W. J., Ab initio molecular orbital theory. Accounts of Chemical Research, 9 (11), 1976, pp 399-406.

[66] M. J. Frisch, G. W. T., H. B. Schlegel, G. E. Scuseria, M. A. Robb, J. R. Cheeseman, G. Scalmani, V. Barone, G. A. Petersson, H. Nakatsuji, X. Li, M. Caricato, A. Marenich, J. Bloino, B. G. Janesko, R. Gomperts, B. Mennucci, H. P. Hratchian, J. V. Ortiz, A. F. Izmaylov, J. L. Sonnenberg, D. Williams-Young, F. Ding, F. Lipparini, F. Egidi, J. Goings, B. Peng, A. Petrone, T. Henderson, D. Ranasinghe, V. G. Zakrzewski, J. Gao, N. Rega, G. Zheng, W. Liang, M. Hada, M. Ehara, K. Toyota, R. Fukuda, J. Hasegawa, M. Ishida, T. Nakajima, Y. Honda, O. Kitao, H. Nakai, T. Vreven, K. Throssell, J. A. Montgomery, Jr., J. E. Peralta, F. Ogliaro, M. Bearpark, J. J. Heyd, E. Brothers, K. N. Kudin, V. N. Staroverov, T. Keith, R. Kobayashi, J. Normand, K. Raghavachari, A. Rendell, J. C. Burant, S. S. Iyengar, J. Tomasi, M. Cossi, J. M. Millam, M. Klene, C. Adamo, R. Cammi, J. W. Ochterski, R. L. Martin, K. Morokuma, O. Farkas, J. B. Foresman, and D. J. Fox, Gaussian 09, Revision A.02. Gaussian, Inc., 2016.

[67] Kobayashi, K., First-principles study of the electronic properties of transition metal nitride surfaces. Surface Science, 493 (1-3), 2001, pp 665-670.

[68] Kamran, S.; Chen, K.; Chen, L., Ab initio examination of ductility features of fcc metals. Physical Review B, 79 (2), 2009, p 024106.

[69] Sun, G.; Kürti, J.; Rajczy, P.; Kertesz, M.; Hafner, J.; Kresse, G., Performance of the Vienna ab initio simulation package (VASP) in chemical applications. Journal of Molecular Structure: THEOCHEM, 624 (1-3), 2003, pp 37-45.

[70] Robertson, J.; Xiong, K.; Clark, S., Band gaps and defect levels in functional oxides. Thin Solid Films, 496 (1), 2006, pp 1-7.

[71] Becke, A., Completely numerical calculations on diatomic molecules in the localdensity approximation. Physical Review A, 33 (4), 1986, p 2786. 
[72] Bernardini, F.; Fiorentini, V., Electronic dielectric constants of insulators calculated by the polarization method. Physical Review B, 58 (23), 1998, p 15292.

[73] Shishkin, M.; Marsman, M.; Kresse, G., Accurate quasiparticle spectra from selfconsistent GW calculations with vertex corrections. Physical Review Letters, 99 (24), 2007, p 246403.

[74] Williamson, A.; Hood, R. Q.; Needs, R.; Rajagopal, G., Diffusion quantum Monte Carlo calculations of the excited states of silicon. Physical Review B, 57 (19), 1998, p 12140.

[75] Mitáš, L.; Martin, R. M., Quantum Monte Carlo of nitrogen: atom, dimer, atomic, and molecular solids. Physical Review Letters, 72 (15), 1994, p 2438.

[76] Paulus, B., The method of increments-a wavefunction-based ab initio correlation method for solids. Physics reports, 428 (1), 2006, pp 1-52.

[77] Shi, N.; Ramprasad, R., Local properties at interfaces in nanodielectrics: An ab initio computational study. IEEE Transactions on Dielectrics and Electrical Insulation, 15 (1), 2008, pp 170-177.

[78] Shi, N. Properties of nanoscale dielectrics from first principles computations. PhD Thesis, University of Connecticut, 2008.

[79] Vansant, E. F.; Van Der Voort, P.; Vrancken, K. C., Characterization and chemical modification of the silica surface Elsevier: 1995; Vol. 93.

[80] Mottola, H. A.; Steinmetz, J. R., Chemically Modified Surfaces: Proceedings of the Fourth Symposium on Chemically Modified Surfaces, Chadds Ford, Pennsylvania, USA, July 31-August 2, 1991 Elsevier Science Limited: 1992; Vol. 4.

[81] Leyden, D. E., Silanes, Surfaces, and Interfaces: Proceedings of the Silanes, Surfaces, and Interfaces Symposium Snowmass, Colorado, June 19-21, 1985 Gordon \& Breach Science Pub: 1986; Vol. 1.

[82] Blitz, J. P.; Murthy, R. S.; Leyden, D. E., Studies of silylation of Cab-O-Sil with methoxymethylsilanes by diffuse reflectance FTIR spectroscopy. Journal of Colloid and Interface Science, 121 (1), 1988, pp 63-69.

[83] Arkles, B., Tailoring surfaces with silanes. 1977.

[84] Lazghab, M.; Saleh, K.; Guigon, P., Functionalisation of porous silica powders in a fluidised-bed reactor with glycidoxypropyltrimethoxysilane (GPTMS) and aminopropyltriethoxysilane (APTES). Chemical engineering research and design, 88 (5-6), 2010, pp 686-692.

[85] Van Bui, H.; Grillo, F.; Van Ommen, J., Atomic and molecular layer deposition: off the beaten track. Chemical Communications, 53 (1), 2017, pp 45-71.

[86] van Ommen, J. R.; Goulas, A., Atomic layer deposition on particulate materials. Materials Today Chemistry, 14, 2019, p 100183.

[87] Puurunen, R. L., Surface chemistry of atomic layer deposition: A case study for the trimethylaluminum/water process. Journal of Applied Physics, 97 (12), 2005, p 9.

[88] Vasudevan, S. A.; Xu, Y.; Karwal, S.; van Ostaay, H. G.; Meesters, G. M.; Talebi, M.; Sudhölter, E. J.; van Ommen, J. R., Controlled release from protein particles encapsulated by molecular layer deposition. Chemical Communications, 51 (63), 2015, pp 12540-12543.

[89] Wank, J. R.; George, S. M.; Weimer, A. W., Coating fine nickel particles with Al2O3 utilizing an atomic layer deposition-fluidized bed reactor (ALD-FBR). Journal of the American Ceramic Society, 87 (4), 2004, pp 762-765. 
[90] Miikkulainen, V.; Leskelä, M.; Ritala, M.; Puurunen, R. L., Crystallinity of inorganic films grown by atomic layer deposition: Overview and general trends. Journal of Applied Physics, 113 (2), 2013, p 2.

[91] George, S. M., Atomic layer deposition: an overview. Chemical Reviews, 110 (1), 2010, pp 111-131.

[92] La Zara, D.; Bailey, M. R.; Hagedoorn, P.-L.; Benz, D.; Quayle, M. J.; Folestad, S.; van Ommen, J. R., Sub-nanoscale Surface Engineering of TiO2 Nanoparticles by Molecular Layer Deposition of Poly(ethylene terephthalate) for Suppressing Photoactivity and Enhancing Dispersibility. ACS Applied Nano Materials, 2020.

[93] Adamczyk, N. M.; Dameron, A. A.; George, S. M., Molecular Layer Deposition of Poly(p-phenylene terephthalamide) Films Using Terephthaloyl Chloride and pPhenylenediamine. Langmuir, 24 (5), 2008, pp 2081-2089.

[94] Liang, X.; Weimer, A. W., Photoactivity passivation of TiO 2 nanoparticles using molecular layer deposited (MLD) polymer films. Journal of Nanoparticle Research, 12 (1), 2010, pp 135-142.

[95] Chen, Y.; Zhang, B.; Gao, Z.; Chen, C.; Zhao, S.; Qin, Y., Functionalization of multiwalled carbon nanotubes with uniform polyurea coatings by molecular layer deposition. Carbon, 82, 2015, pp 470-478.

[96] Zhou, Y.; Peng, S.; Hu, J.; He, J., Polymeric insulation materials for HVDC cables: Development, challenges and future perspective. IEEE Transactions on Dielectrics and Electrical Insulation, 24 (3), 2017, pp 1308-1318.

[97] Khalil, M. S., International research and development trends and problems of HVDC cables with polymeric insulation. IEEE Electrical Insulation Magazine, 13 (6), 1997, pp 35-47.

[98] Andrews, T.; Hampton, R.; Smedberg, A.; Wald, D.; Waschk, V.; Weissenberg, W., The role of degassing in XLPE power cable manufacture. IEEE Electrical Insulation Magazine, 22 (6), 2006, pp 5-16.

[99] Hanley, T. L.; Burford, R. P.; Fleming, R. J.; Barber, K. W., A general review of polymeric insulation for use in HVDC cables. IEEE Electrical Insulation Magazine, 19 (1), 2003, pp 13-24.

[100] Ghorbani, H.; Jeroense, M.; Olsson, C.; Saltzer, M., HVDC Cable SystemsHighlighting Extruded Technology. IEEE Transactions on Power Delivery, 29 (1), 2014, pp 414-421.

[101] Andritsch, T.; Vaughan, A.; Stevens, G. C., Novel insulation materials for high voltage cable systems. IEEE Electrical Insulation Magazine, 33 (4), 2017, pp 27-33.

[102] Hirai, N.; Minami, R.; Tanaka, T.; Ohki, Y., Chemical group in crosslinking byproducts responsible for charge trapping in polyethylene. IEEE Transactions on Dielectrics and Electrical Insulation, 10 (2), 2003, pp 320-330.

[103] Singh, P.; Déparrois, N.; Burra, K.; Bhattacharya, S.; Gupta, A., Energy recovery from cross-linked polyethylene wastes using pyrolysis and $\mathrm{CO} 2$ assisted gasification. Applied Energy, 254, 2019, p 113722.

[104] Shang, L.; Wang, S.; Zhang, Y.; Zhang, Y., Pyrolyzed wax from recycled cross-linked polyethylene as warm mix asphalt (WMA) additive for SBS modified asphalt. Construction and Building Materials, 25 (2), 2011, pp 886-891. 
[105] Qi, X.; Boggs, S., Thermal and mechanical properties of EPR and XLPE cable compounds. IEEE Electrical Insulation Magazine, 22 (3), 2006, pp 19-24.

[106] Zhou, Y.; He, J.; Hu, J.; Huang, X.; Jiang, P., Evaluation of polypropylene/polyolefin elastomer blends for potential recyclable HVDC cable insulation applications. IEEE Transactions on Dielectrics and Electrical Insulation, 22 (2), 2015, pp 673-681.

[107] Hosier, I. L.; Vaughan, A. S.; Swingler, S. G., An investigation of the potential of polypropylene and its blends for use in recyclable high voltage cable insulation systems. Journal of Materials Science, 46 (11), 2011, pp 4058-4070.

[108] Jiang, W.; Tjong, S.; Li, R., Brittle-tough transition in PP/EPDM blends: effects of interparticle distance and tensile deformation speed. Polymer, 41 (9), 2000, pp 3479-3482.

[109] Du, B. X.; Xu, H.; Li, J.; Li, Z., Space charge behaviors of PP/POE/ZnO nanocomposites for HVDC cables. IEEE Transactions on Dielectrics and Electrical Insulation, 23 (5), 2016, pp 3165-3174.

[110] Green, C. D.; Vaughan, A. S.; Stevens, G. C.; Pye, A.; Sutton, S. J.; Geussens, T.; Fairhurst, M. J., Thermoplastic cable insulation comprising a blend of isotactic polypropylene and a propylene-ethylene copolymer. IEEE Transactions on Dielectrics and Electrical Insulation, 22 (2), 2015, pp 639-648.

[111] Diao, J.; Huang, X.; Jia, Q.; Liu, F.; Jiang, P., Thermoplastic isotactic polypropylene/ethylene-octene polyolefin copolymer nanocomposite for recyclable HVDC cable insulation. IEEE Transactions on Dielectrics and Electrical Insulation, 24 (3), 2017, pp 1416-1429. 
CHAPTER 3

\author{
Amino Functionalized Silica \\ Nanoparticles in Polypropylene- \\ Based Nanodielectrics
}




\begin{abstract}
$^{1}$
The effect of filler surface functionalization with 3-aminopropyltriethoxysilane (APTES) on the charge trapping and transport was studied in polypropylene (PP)/(ethyleneoctene) copolymer (EOC)/silica nanodielectrics. Different reaction conditions were utilized for silica functionalization to alter the deposited layer morphology. This approach made it possible to engineer the filler-polymer interface to achieve optimized dielectric properties for the nanocomposites. The successful chemical modification of the silica surface was confirmed via Thermogravimetric Analysis (TGA), Fourier Transform Infrared Spectroscopy (FTIR) and X-Ray Photoelectron Spectroscopy (XPS). Subsequently, the effect of the engineered filler-polymer interface on the nanocomposites' crystallinity was analyzed with Differential Scanning Calorimetry (DSC). Scanning Electron Microscopy (SEM) was utilized to observe the morphology of the nanocomposite as well as the silica dispersion. Finally, the effect of the silica functionalization on the dielectric properties of PP/EOC/silica nanocomposites was tested via Thermally Stimulated Depolarization Current (TSDC) and Broadband Dielectric Spectroscopy (BDS). The results suggested that the presence of the amine functionality on the silica reduces interfacial losses in nanocomposites, and hinders further injection of space charge by introducing deep trap states at the filler-polymer interface. Under certain conditions, APTES can form an "island-like" morphology on the silica surface. These islands can facilitate nucleation, inducing transcrystallization at the filler-polymer interface. The "island-like" structures present on the silica would further contribute to the induction of deep traps at the filler-polymer interface resulting in reduction of space charge injection.
\end{abstract}

1 The results of this chapter are published in ACS Applied Polymer Materials, 2, 8, pp. 3148-3160, 2020, (https://doi.org/10.1021/acsapm.0c00349) 


\section{Introduction}

Incorporation of nanoparticles (NPs) in dielectric materials to enhance their insulating properties has been studied extensively in the recent years ${ }^{1-4}$. Nanocomposites (NCs) containing various fillers including semiconducting $\left(\mathrm{ZnO}^{5}\right.$ and $\mathrm{TiO}^{6}{ }^{6}$ ) and insulating particles $\left(\mathrm{MgO}^{7}, \mathrm{SiO}^{8}\right)$, have been reported in literature, exhibiting substantial enhancements in e.g. dielectric strength and endurance under high voltages ${ }^{9}$. These improvements can be due to numerous phenomena, mainly stemming from the large interfacial area introduced by nanofillers incorporated into the polymeric matrix ${ }^{10}$ as well as the alteration of the polymer/filler interface properties ${ }^{9}$. The later can be controlled by changing the type and surface chemistry of the NPs. Due to the polar surface of the above mentioned NPs, these are prone to adsorb moisture and other polar species (e.g. byproducts of production and surface treatment), which can be detrimental to the dielectric properties ${ }^{11-12}$. In this regard, NPs such as fumed silica are more favorable due to their low moisture and high purity. Moreover, it has been shown that silica filled NCs exhibit high electron-phonon interaction at their interface with the surrounding polymer chains ${ }^{13}$, which is another reason to favor fumed silica over other available NPs. In order to improve their dispersion and tailor the filler-polymer interfacial properties, the filler surface can be modified with certain functional groups. This can influence nucleation and crystallinity ${ }^{14}$, mobility of species ${ }^{15}$ and entanglement density in the interfacial region ${ }^{16-17}$. Consequently, changes in the NPs' surface properties can lead to improvements of the dielectric properties of the NCs ${ }^{18}$.

Functionalization of NPs can improve the dielectric properties of the NCs by enhancing the filler dispersion in the polymeric matrix: in case of a DC field, due to the differences in electrical conductivity of the composite's components, the electric field tends to bend towards the particles leading to local electric field concentration. Furthermore, due to the polarization of interfaces, an additional long-range attractive force will be imposed on the charge carriers and draws them to the NPs ${ }^{19}$. So, in fact, NPs act as electrical defect centers in polymer nanodielectrics ${ }^{20}$. The extent of this local field concentration, however, is not only a factor of the difference in permittivity (in case of AC fields) or 
conductivity (in case of DC) between the particles and the polymer matrix, but also of the particle size, their aggregation and agglomeration ${ }^{1}$. Improving the dispersion of the nanofillers can lead to improvement of the dielectric breakdown strength. However, filler dispersion is difficult to control and tends to vary significantly as it is affected by several factors e.g. surface energy of the filler, polymer melt viscosity and processing conditions ${ }^{21-}$ 22.

Another phenomenon through which surface modification of the NPs can affect the dielectric properties of the NCs, is alteration of the electron-phonon interactions at the filler-polymer interface. This can be achieved e.g. by the introduction of polar functional groups at the interface ${ }^{23-24}$. Siddabattuni et al. ${ }^{25}$ investigated NCs based on epoxy resin, $\mathrm{TiO}_{2}$ and $\mathrm{BaTiO}_{3} \mathrm{NPs}$, and observed that upon introduction of electron-withdrawing phenyl groups at the polymer-particle interface, the leakage current and dielectric loss was significantly reduced. This also led to an improvement in dielectric breakdown strength. Huang et al. ${ }^{2}$ noticed that the amount of space charges in LLDPE/silica nanodielectrics was decreased by the attachment of dimethyldichlorosilane to the surface of the silica NPs. Dongling et al. ${ }^{26}$ reported a much higher dielectric breakdown strength and improved space charge distribution by introduction of amine functional groups to the surface of $\mathrm{TiO}_{2} \mathrm{NPs}$ incorporated in an LDPE matrix. Furthermore, the density of the trap states, and as a result, the space charge distribution can be altered upon grafting species with polar functional groups onto the NP surface, influencing the mobility of charges by introduction of shallow trap sites ${ }^{27}$ or reducing the number of deep trapped charges ${ }^{1}$ in the filler-polymer interaction zones. Yamano et al. ${ }^{28}$ found that the addition of $5 \times 10^{-6} \mathrm{~mol} \mathrm{~g}^{-1}$ of an azocompound increased the breakdown strength of LDPE by about $200 \mathrm{MV} \mathrm{m}^{-1}$ at temperatures lower than $30^{\circ} \mathrm{C}$. They attributed this increase to either the trapping or the excitation effect of the azocompounds, which acted as electric dipoles. Accordingly, it can be hypothesized that attaching a polar functionality to the surface of the NPs can induce this sort of electronic behavior, as the dipoles at the particle-polymer interface may affect charge carrier transport and trapping ${ }^{4}$. Recent studies have shown that the localized states (traps) in polymer dielectrics have substantial effects on the charge transport under a high 
field, and can be closely addressed to the macroscopic dielectric properties including charge injection, space charge distribution, and mobility of charge carriers ${ }^{29-30}$. Therefore, it is expected that adding certain polar chemical functionalities to the polymer-filler interface will introduce new electronic states and alter the spatial and energy distribution of the localized states in the system, which may account for the changes in dielectric properties of nanofilled dielectrics.

Space charge behavior in an insulating polymer is also closely related to carrier traps located at the interfaces that are induced as a result of crystallization in semi-crystalline polymers ${ }^{31}$. The morphology of these interfaces would significantly affect trap density, trap depth distribution, and charge carrier mobility ${ }^{32}$. Therefore, by engineering the crystallinity of a nanodielectric, it is possible to control the aforementioned properties. A promising way to control crystallinity in a polymer NC is through engineering the surface properties of the NPs. Grafting polymers or silane modifying agents onto the silica surface has been proven to effectively improve its nucleating ability which results in higher spherulite density within the NC ${ }^{33-34}$. This could have beneficial influences on the amount and distribution of the space charges.

The morphological changes in NCs can also be a result of polymer blending which has recently become especially relevant for new polypropylene (PP)-based HVDC cable insulation materials for improving certain mechanical properties. While PP has shown promising dielectric performance, its inherent rigidity, especially at low temperatures, hinders its utilization for this application ${ }^{35-36}$. This necessitates blending PP with another polymer with more suitable mechanical properties such as polyolefin elastomers. In regards to NC systems with a polymer blend as the matrix, studying the crystallinity and morphology effects is of great importance, especially when the NPs have been subjected to surface modification.

In this study, silica NPs were modified with 3-aminopropyltriethoxysilane (APTES), a polar modifying agent, and compounded with PP/EOC polymer blends. The aim of surface modification was twofold: On the one hand, it is speculated that the amine functional group in APTES is prone to alter the interactions and electronic features at the silica-polymer 
interface. As a consequence, trapping properties of the interface undergoes meaningful changes, which might induce beneficial influences on the bulk insulation characteristics of the NC materials. On the other hand, by engineering the nanofiller surfaces, their nucleating ability and hence the crystallinity of the nanodielectric can be controlled. This way, an optimized modification reaction to control the trap states and the space charge phenomena could be developed. Firstly, the silica modification is evaluated using Thermogravimetry Analysis (TGA), Fourier Transform Infrared (FTIR) Spectroscopy, X-ray Photoelectron Spectroscopy (XPS) and Transmission Electron Microscopy (TEM). Subsequently, the influence of silica modification on the dielectric response of the material is investigated with Broadband Dielectric Spectroscopy (BDS). Charge trapping properties of the aforementioned NCs are also studied via Thermally Stimulated Depolarization Current (TSDC) measurements. Since the charge trapping behavior can be significantly influenced by the crystalline structure, Differential Scanning Calorimetry (DSC) was performed to study the crystalline structure in detail.

\section{Experimental Section}

\subsection{Silica Modification}

A high purity, low moisture content fumed silica grade, AEROSIL 200 (Evonik Industries, Germany) was used as the filler for this study. The silica functionalization was carried out in liquid phase (toluene, BOOM Chemicals, NL, 99\%) using 3aminopropyltriethoxysilane (APTES) as the modifying agent (Sigma Aldrich, 99\%). The reaction took place in a glass round bottom flask immersed in an oil heating bath and equipped with a mechanical stirrer, cooler and thermometer. The reaction conditions were varied in order to control the amount and morphology of the grafted silane on the silica. Temperature, time and the amount of added water were chosen as the most effective parameters. Reactions were carried out at $20^{\circ} \mathrm{C}$ and $80^{\circ} \mathrm{C}$, for 1 or 24 hours, with and without a stoichiometric amount of water for hydrolysis of the silane. The APTES content in the solution was calculated as twice the amount needed for covering the silica with a monolayer. The details of the modification reactions are depicted in Table 3.1. In general, anhydrous systems with only a trace of water and low silane concentrations are desirable 
for the preparation of smooth APTES-derived silane layers ${ }^{37}$. Silica, silane and additional water were mixed with toluene as the medium of the reaction. The purity of the toluene was $98 \%$, and it was used without further purification. With water present in the reaction, hydrolysis and subsequently condensation of APTES is very likely. Therefore, different APTES film morphologies can be expected, depending on whether or not water is involved in the reaction. Figure 3.1 represents the APTES hydrolysis and its grafting onto the silica surface with and without condensation.

a.

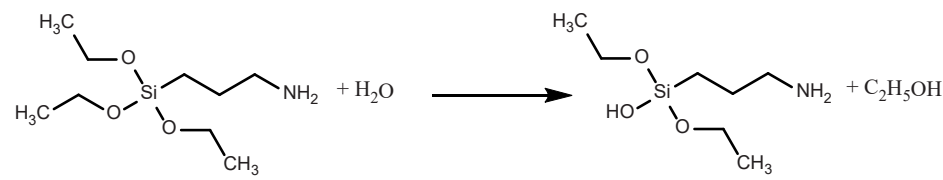

b.

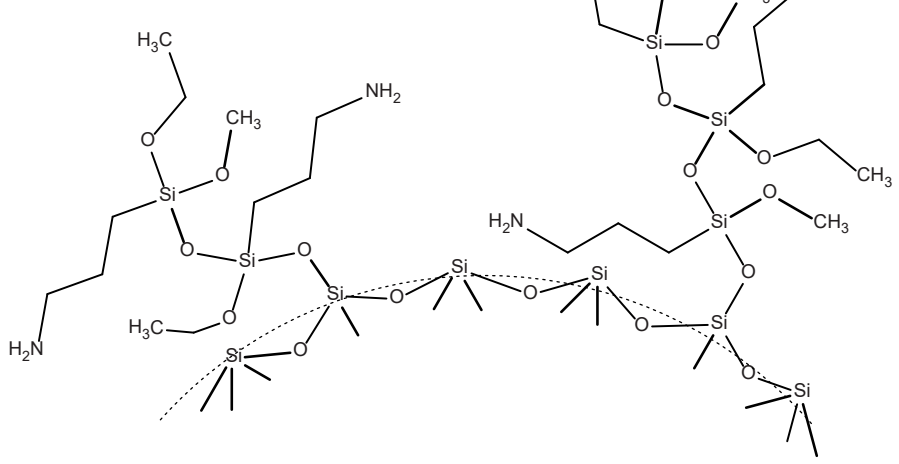

C.

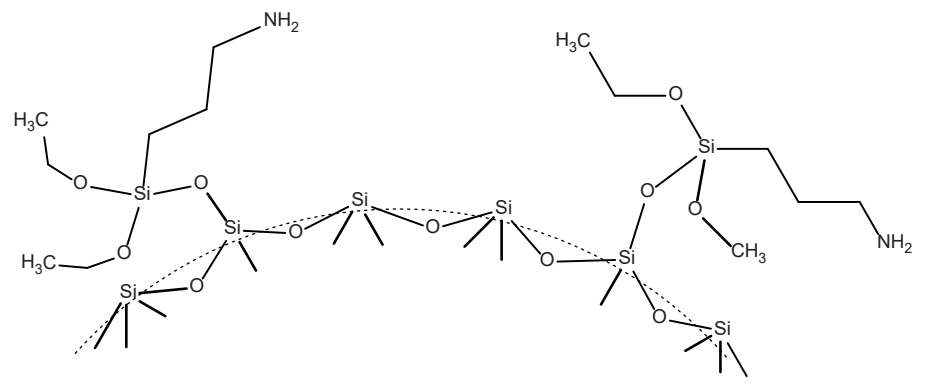

Figure 3.1. a: Hydrolysis and reaction of APTES with silica, b: with and c: without condensation 
Table 3.1. Reaction conditions utilized for modification of the silica NPs

\begin{tabular}{|c|c|c|c|c|}
\hline & $\begin{array}{l}\text { Sample } \\
\text { Number }\end{array}$ & $\begin{array}{c}\text { Temperature } \\
\left({ }^{\circ} \mathrm{C}\right)\end{array}$ & $\begin{array}{c}\text { Time } \\
\text { (hours) }\end{array}$ & $\begin{array}{c}\text { Added } \\
\text { Water } \\
\text { (grams) }\end{array}$ \\
\hline & 0 & \multicolumn{3}{|c|}{ Untreated Silica } \\
\hline \multirow{4}{*}{$\begin{array}{l}\text { W/o } \\
\text { Water }\end{array}$} & 1 & 20 & 1 & 0 \\
\hline & 2 & 80 & 1 & 0 \\
\hline & 3 & 20 & 24 & 0 \\
\hline & 4 & 80 & 24 & 0 \\
\hline \multirow{4}{*}{$\begin{array}{l}\text { W/ } \\
\text { Water }\end{array}$} & 5 & 20 & 1 & 1.8 \\
\hline & 6 & 80 & 1 & 1.8 \\
\hline & 7 & 20 & 24 & 1.8 \\
\hline & 8 & 80 & 24 & 1.8 \\
\hline
\end{tabular}

\subsection{Preparation of the Nanocomposites}

NCs based on polypropylene (PP, 3 w\% ethylene)/ethylene-octene copolymer (EOC, 17 w\% octene) blends filled with functionalized silica NPs were prepared by adding $1 \mathrm{w} \%$ of the silica as well as $0.3 \mathrm{w} \%$ of an antioxidant package to the polymer matrix in a twin-screw micro extruder (Haake MiniLab Rheomix CTW5, Thermo Fisher Scientific, Waltham, Massachusetts, USA) at $230{ }^{\circ} \mathrm{C}$ and $100 \mathrm{rpm}$, and subsequent injection of the molten compound into a square mold at $60^{\circ} \mathrm{C}$ using a Haake MiniJet Pro Piston Injection Moulding System (Thermo Fisher Scientific, Waltham, Massachusetts, USA). The mold dimensions were $26 \times 26 \times 0.5 \mathrm{~mm}$. The nanocomposite samples were numbered according to the silica sample incorporated. For instance, NC_1 nanocomposite is filled with silica sample number 1.

\subsection{Silica Characterization}

Thermogravimetric Analysis (TGA) was utilized to quantify the level of modification. The silica samples were heated from room temperature to $850^{\circ} \mathrm{C}$ in air atmosphere with a 
rate of $20^{\circ} \mathrm{C} / \mathrm{min}$ while the mass loss of the sample being measured. The mass loss between $300-850{ }^{\circ} \mathrm{C}$ can be attributed to thermal decomposition of APTES chemically attached to the silica surface ${ }^{38}$. Fourier transform infrared (FTIR) spectroscopy was utilized in diffuse reflectance mode using a Perkin Elmer Spectrum 100 with a diffuse reflectance accessory. The samples were prepared using potassium bromide $(\mathrm{KBr}$; 99+\%, FTIR grade, Harrick Scientific Corporation) as background. Spectra were recorded from $4000-400 \mathrm{~cm}^{-1}$ and averaged over 128 scans, using a resolution of $4.0 \mathrm{~cm}^{-1}$. All the tests were performed at room temperature. X-ray Photoelectron Spectroscopy (XPS) was conducted by means of a PHI Quantera Scanning X-ray Microscope (USA). This method is based on irradiating a material with a beam of X-rays, while simultaneously measuring the kinetic energy and number of electrons that escape from the surface (up to $10 \mathrm{~nm}$ in depth) of the material being analyzed. This way, it is possible to determine the atomic composition of the deposited film on the silica. Transmission Electron Microscopy (TEM) including EnergyFiltering mode (EFTEM) was done on the modified silica NPs, using a Philips CM300ST-FEG Transmission Electron Microscope in order to take a closer look at the modified silica surface.

\subsection{Thermally Stimulated Depolarization Current (TSDC)}

Thermally Stimulated Depolarization Current (TSDC) was utilized to analyze the charge trapping and transport phenomena and space charge formation in the NCs under a direct current (DC) field. The samples were prepared by depositing circular gold (Au) electrodes (100 nm in thickness) on both sides of each NC specimen by e-beam evaporation under high vacuum. Subsequently, the samples were short circuited and kept in a vacuum desiccator overnight prior to the measurements to remove any stored charge during evaporation. The TSDC tests were performed by rapidly heating up the NCs to the poling temperature of $70{ }^{\circ} \mathrm{C}$, and then a $3 \mathrm{kV} / \mathrm{mm}$ DC electric field was applied for 20 minutes under isothermal conditions. After the polarization step, the samples were cooled down to $-50{ }^{\circ} \mathrm{C}$ while the electric field was still on. This would force the polarized species and injected charges to remain in the specimen. The samples were then short-circuited and linearly 
heated up to $140{ }^{\circ} \mathrm{C}$ at $3{ }^{\circ} \mathrm{C} / \mathrm{min}$, while the depolarization current being measured by an electrometer 6517B (Keithley Instruments, USA).

\subsection{Broadband Dielectric Spectroscopy (BDS)}

The real and imaginary parts of complex permittivity (as in Equation 3-1) were measured after the TSDC test, using a Novocontrol Alpha-A dielectric analyzer (Germany) within the frequency range of $10^{-2}-10^{6} \mathrm{~Hz}$ and under an applied voltage of $1 \mathrm{~V}$. All the measurements were conducted at room temperature.

$$
\varepsilon_{r}^{*}=\varepsilon_{r}^{\prime}+i \varepsilon_{r}^{\prime \prime}
$$

(Equation 3-1)

\subsection{Morphology and Crystallinity Analysis}

Scanning Electron Microscopy (SEM) was performed on the nanodielectric composites by means of a Zeiss MERLIN HR-SEM (Germany) to study the filler dispersion and polymer blend morphology. The silica size distribution was analyzed using open-source ImageJ software with Trainable Weka Segmentation plugin ${ }^{39}$. Differential Scanning Calorimetry (DSC) was performed by means of a Netzsch DSC 214 Polyma. Specimens were subjected to two heating/cooling cycles from $-50^{\circ} \mathrm{C}$ to $200^{\circ} \mathrm{C}$. The rate of heating/cooling was set at $3{ }^{\circ} \mathrm{C} / \mathrm{min}$, to be comparable to the heating rate in TSDC measurements. Degree of crystallization was calculated using the enthalpy of melting for the $100 \%$ crystalline PP/EOC blend, $249 \mathrm{~J} / \mathrm{g}$. This value was calculated using the $100 \%$ crystalline enthalpy of melting for each component and implementing the law of mixtures.

\section{Results and Discussion}

\subsection{Characterization of Nanoparticles}

The TGA graphs depicted in Figures 3.2a and 3.2c show a higher mass loss for the modified silica samples compared to the unmodified sample, indicating the successful treatment of the silica surface. In order to compare the amount of grafted silane for different samples, the TGA mass loss data was normalized against the value at $300^{\circ} \mathrm{C}$ (Figures $3.2 \mathrm{~b}$ and $3.2 \mathrm{~d}$ ). The mass loss below $300{ }^{\circ} \mathrm{C}$ is related to the removal of nonchemically bonded compounds and volatiles. On the one hand, it can be seen that the mass 
loss ranges between $3.2 \%$ to $4.2 \%$ when the reaction takes place with no additional water in the system. This indicates that the silanization reaction can take place even in the absence of excess water ${ }^{40}$ because of the self-catalyzing nature of APTES due to hydrogen bond interactions of the amine moiety with the silanol groups on silica surface ${ }^{41}$. It also seems, in this case, that the grafting of the APTES is not significantly affected by change in reaction time or temperature. In the presence of excess water, on the other hand, a higher value of weight loss, $6.4 \%$, is achieved for the samples modified for 24 hours (compared to $1 \mathrm{~h}$ ). However, with the reaction time kept constant, no difference is observed in the values of mass loss upon increasing the temperature. The values of silane grafting density in $\mathrm{mmol} / \mathrm{g}$ silica, presented in Figure 3.3, can better demonstrate this observation. These values were calculated based on the TGA results, using Equation 3-2:

$$
\text { APTES Grafting Density }\left(\frac{m m o l}{g \text { silica }}\right)=\frac{\Delta W \times 1000}{M_{w} \times(100-\Delta W)}
$$

Where $\Delta W$ is the mass loss between $300-850^{\circ} \mathrm{C}$ and $M_{w}$ is the molecular weight of APTES, $221.37 \mathrm{~g} / \mathrm{mol}$. 

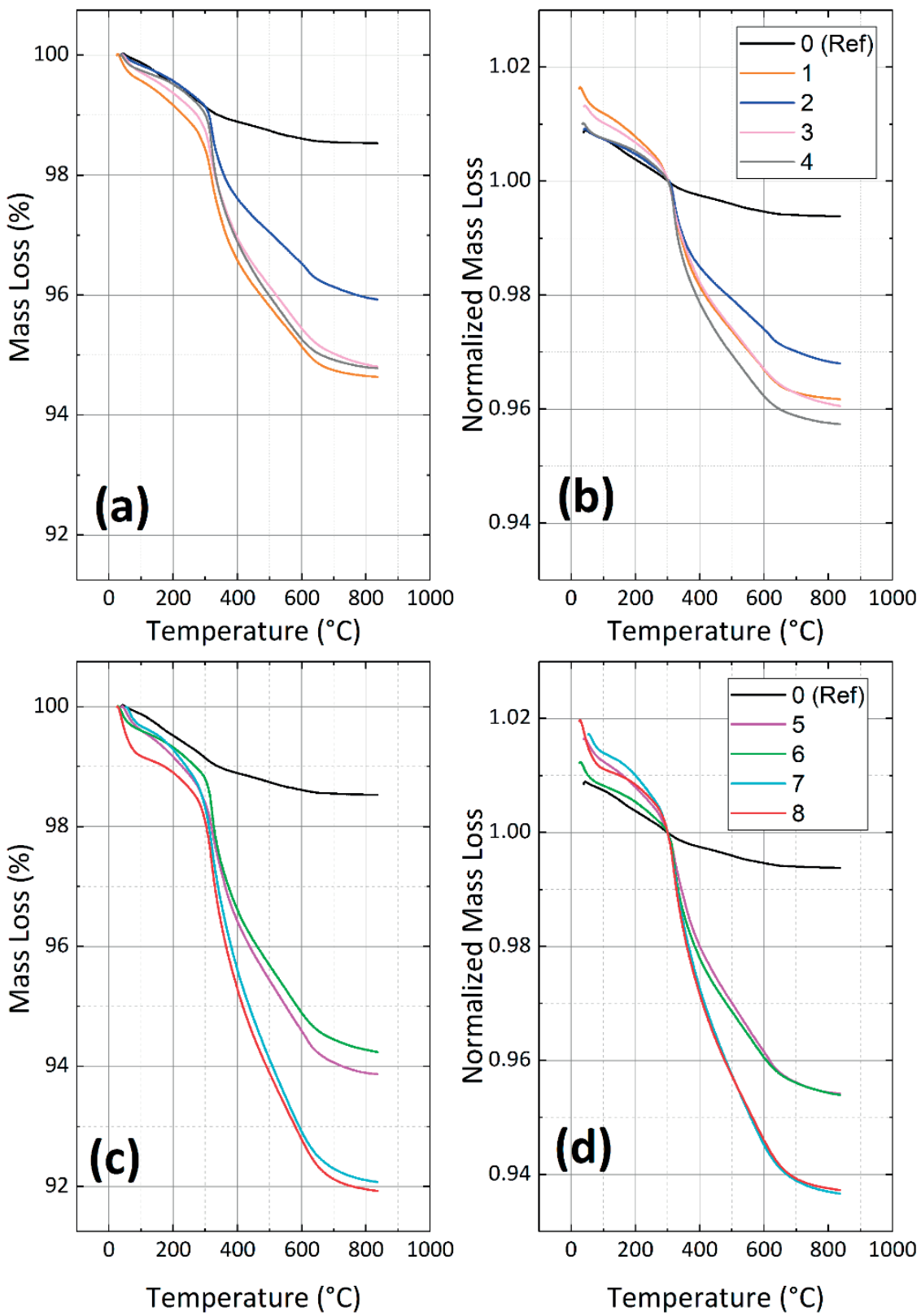

Figure 3.2. a, c: TGA graphs for the modified silica samples compared to the untreated silica; b, d: TGA mass loss normalized to the mass loss at $300^{\circ} \mathrm{C}$ 

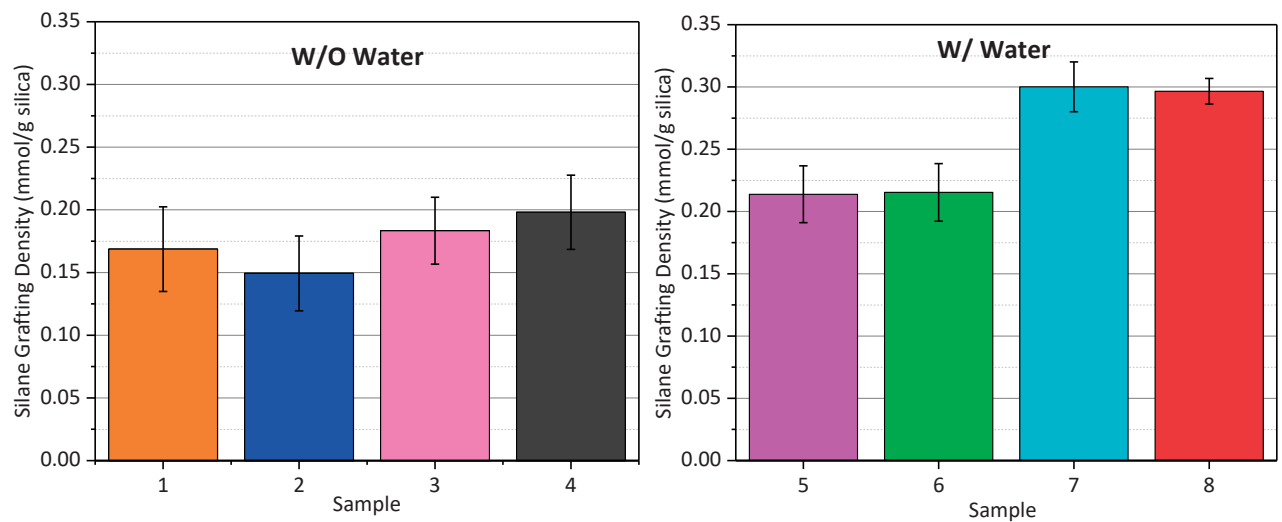

Figure 3.3. Values of silane grafting density for different modified silica samples

FTIR spectra of the modified silica NPs also confirm the chemical attachment of the APTES to the silica surface. The spectra for all the studied samples are presented in Figure 3.4. The bands at $3300 \mathrm{~cm}^{-1}, 2850 \mathrm{~cm}^{-1}$ and $1600 \mathrm{~cm}^{-1}$ are corresponding to $\mathrm{N}-\mathrm{H}$ stretching, $\mathrm{C}-\mathrm{H}$ stretching and $\mathrm{N}-\mathrm{H}$ bending, respectively. This proves the presence of chemically bonded APTES on the silica surface. The sharp band at $3700 \mathrm{~cm}^{-1}$ (isolated $-\mathrm{OH}$ ) and the broad band at $3500 \mathrm{~cm}^{-1}$ representing the free and intermolecular hydroxyl groups almost completely vanished upon modification. This significant difference can indicate that the surface of the modified silica is less hydrophilic and well covered by the chemically attached silane modifying agent. 

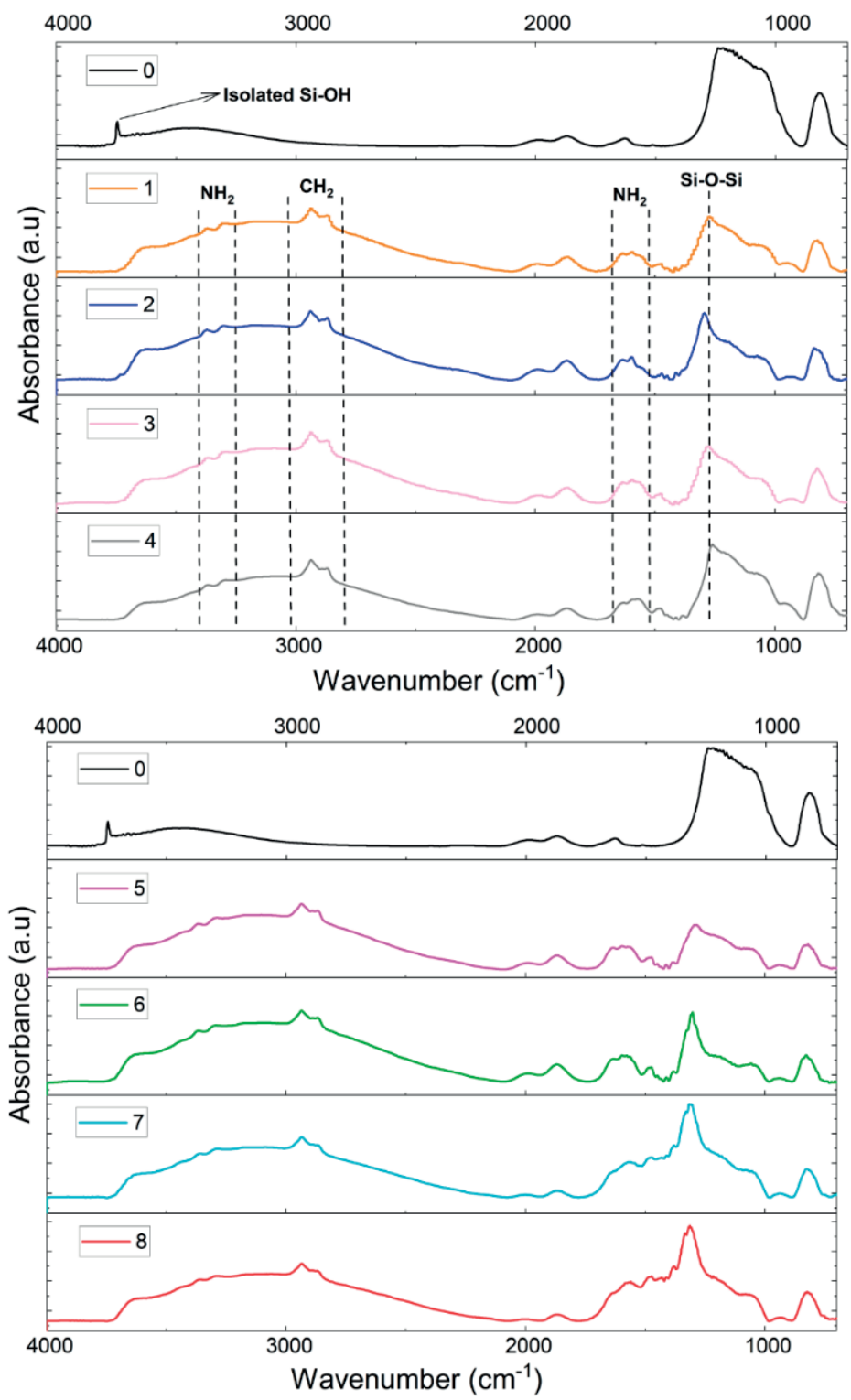

Figure 3.4. FTIR spectra of the untreated and treated silica samples

The XPS spectra of Sample 8 is presented in Figure 3.5a along with the spectra from the untreated silica for comparison. The emergence of the peaks corresponding to $\mathrm{C} 1 \mathrm{~s}$ and N1s in Sample 8 confirms the presence of APTES on silica surface. The small C1s peak in the reference sample is present due to contaminations or traces of carbon dioxide in the equipment chamber. Elemental fine scans in Figure 3.5b indicate the atomic environment 
and are dependent on the chemical bonds in the film. As expected, the N1s fine scan reveals that there are two peaks at $398.2 \mathrm{eV}$ and $401.3 \mathrm{eV}$, indicative of nitrogen bonded with carbon and hydrogen, respectively. The C1s scan also exhibits two peaks at $284.8 \mathrm{eV}$ and $286.0 \mathrm{eV}$. These peaks represent two types of carbon bonds in the APTES film, namely C-C and C-N. Therefore, the XPS data also confirm successful deposition of chemically bonded APTES on silica NPs.

a.

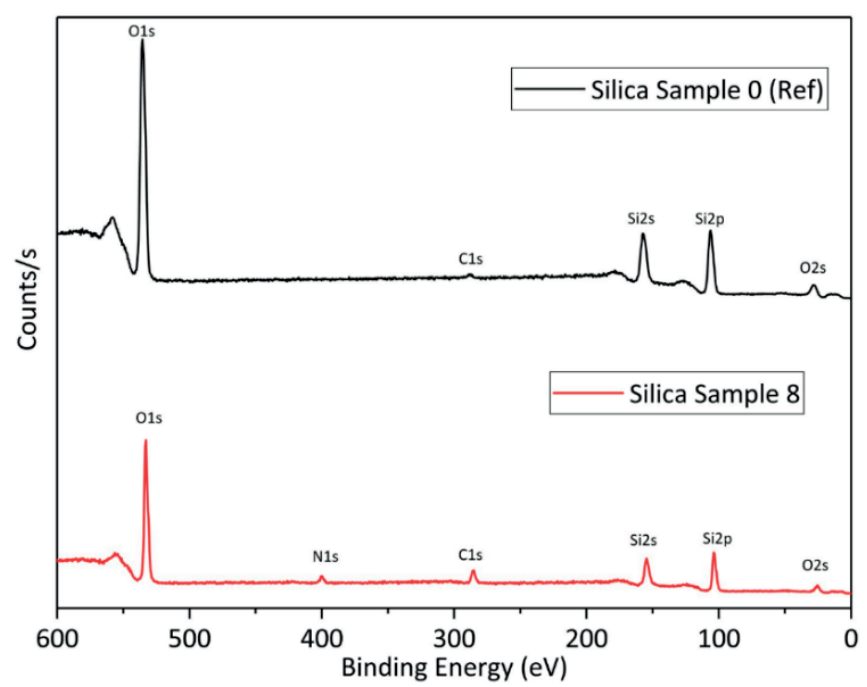

b.
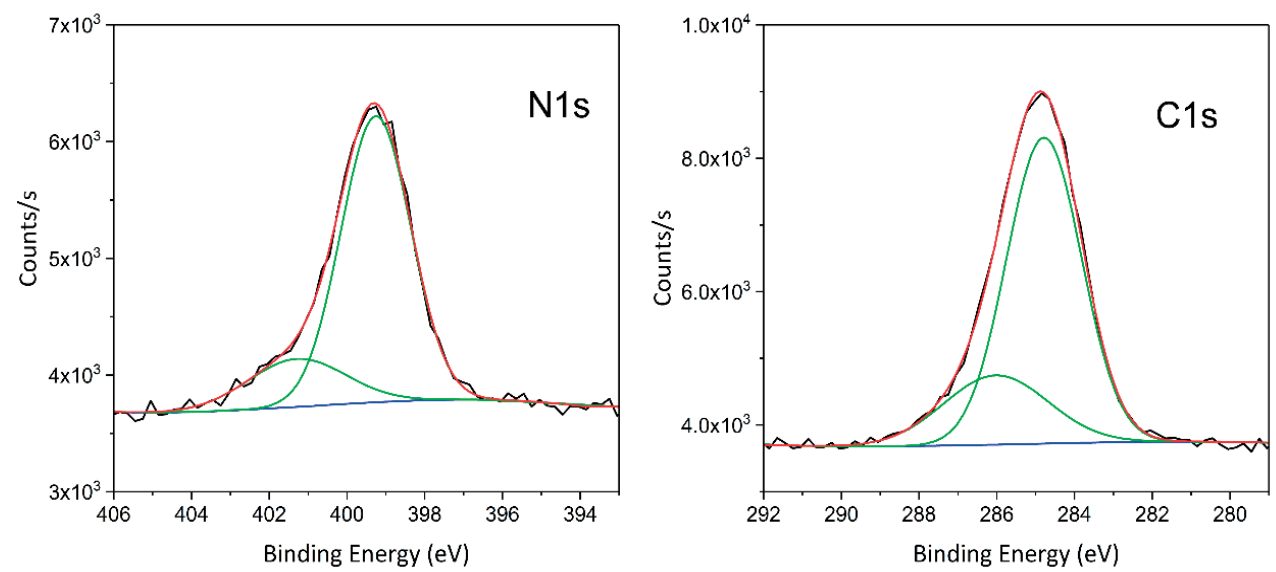

Figure 3.5. a: XPS full spectra of the modified and unmodified silica samples; b: N1s and C1s fine scans of silica Sample 8. 
Energy-filtered transmission electron microscopy (EFTEM) was performed on the silica samples to analyze the silica surface structure after modification via elemental mapping. Carbon mapping of the modified silica in Figure 3.6 shows the APTES propyl chain distributed along the surface, and is indicative of the spatial distribution of the modification layer over the silica cluster.

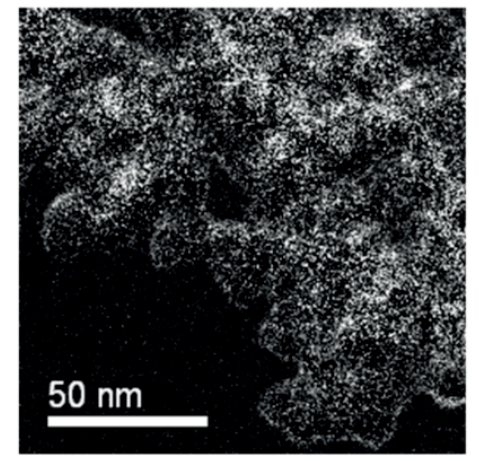

Silica Sample 7

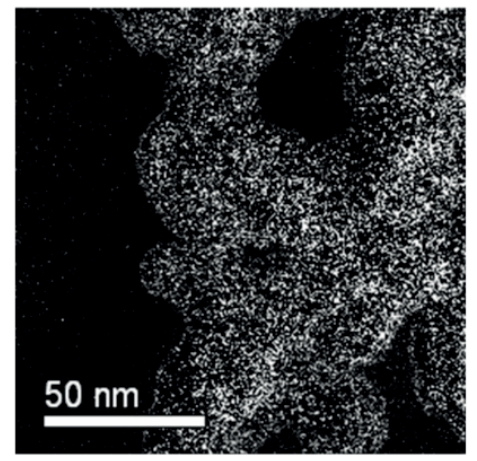

Silica Sample 8

Figure 3.6. EFTEM carbon mapping for silica Samples 7 and 8

\subsection{Characterization of PP/EOC/Silica Nanocomposites}

Treating the silica with APTES can result in less hydrophilic NPs. So, varying the grafting density of APTES can affect the silica dispersion in the polymer matrix. Therefore, filler dispersion in the nanocomposites with low (NC_1 and NC_2) and high (NC_7 and NC_8) levels of APTES grafting density was compared to NC_O (with unmodified silica) using SEM. The corresponding images are depicted in Figure 3.7. It is observed that the polymer matrix exhibits a well distributed two phase morphology: a well dispersed sea-island structure with EOC elongated in the direction of the flow. This sort of structure is usually observed in immiscible injection molded polymer blends. When the molten material enters the cavity in a parabolic profile, the material in the front, undergoing considerable stretching, is deposited on the wall as an immobile frozen layer. Subsequently, as the rest of the molten material flows through this envelope, the dispersed phase is elongated in the direction of the flow ${ }^{42-43}$. Despite the immiscibility, the blends of PP with ethylene-based copolymers have been shown to have superior dielectric breakdown strength compared to the each base material ${ }^{44}$. Therefore, the phase separated morphology in our studied 
nanocomposites is presumed to have no deteriorating effect on the dielectric properties of the system. The dispersion histograms in Figure 3.7 also shows that the mean silica cluster size reduces from ca. $300 \mathrm{~nm}$ to $120 \mathrm{~nm}$ upon modification of the NPs. This indicates that modifying the silica with APTES, results in relatively better dispersion of the NPs. Moreover, to the extent SEM can detect, different grafting densities do not significantly influence the dispersion quality of the silica. This can be a side effect of the polarity of the silane modifying agent. The silica clusters are well distributed throughout the matrix, mostly located in the PP phase. This phenomenon is also detected in the DSC results which will be discussed in the following sections.

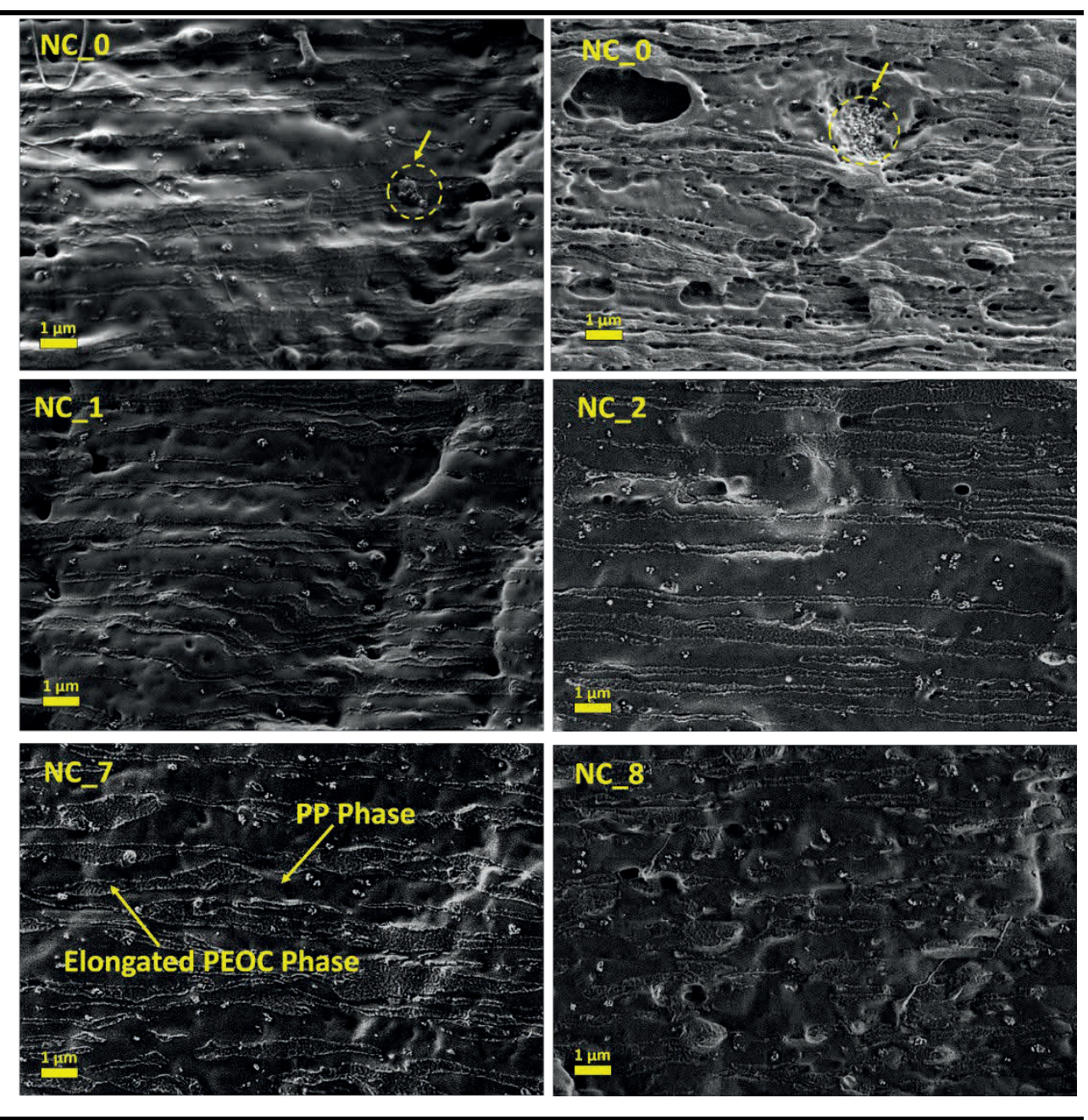




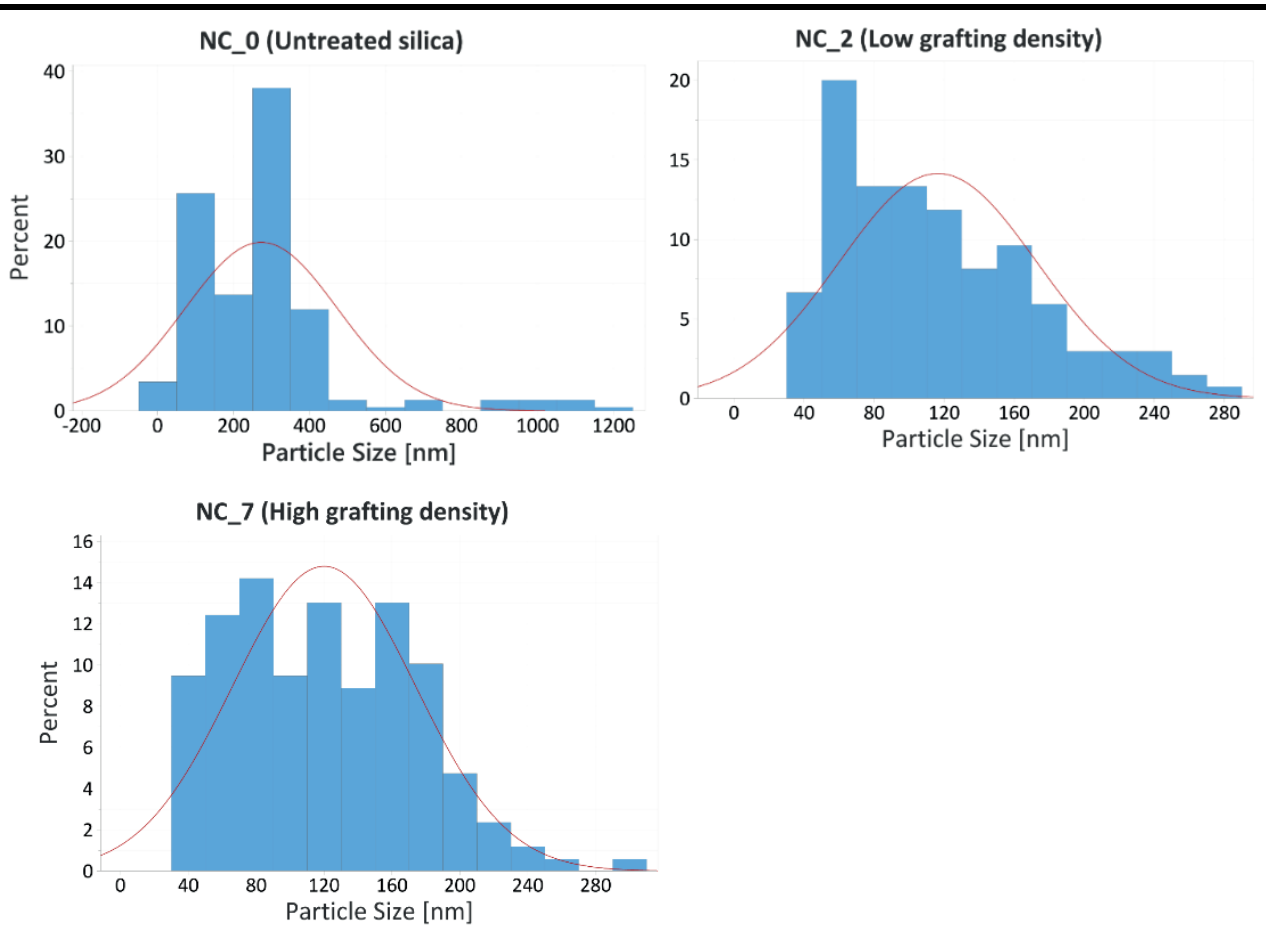

Figure 3.7. SEM images of nanocomposites with untreated silica (NC_0), low (NC_1 and NC_2) and high (NC_7 and NC_8) APTES grafting densities (top). Silica cluster size distribution histograms (bottom). A wide range of particle sizes are observed for NC_0 (mean: $297.7 \mathrm{~nm}$ ), whereas the modified samples exhibit a narrow distribution (mean: $115 \mathrm{~nm}$ ).

Dielectric properties in nanocomposite insulating materials are highly influenced by the dynamics of interfacial interactions in the system. These interactions can affect measurable macroscopic properties such as permittivity and dielectric loss. Therefore, BDS was applied to study the underlying physics of the dielectric phenomena in our studied nanocomposites. Dielectric spectroscopy results are depicted in Figure 3.8 (a,b and c), where real $\left(\varepsilon_{r}{ }^{\prime}\right)$ and imaginary $\left(\varepsilon_{r}^{\prime \prime}\right)$ parts of the complex permittivity as well as the loss tangent $(\tan \delta)$ are plotted against the frequencies of the applied field. Also, in order to further realize the relaxation processes in the nanocomposites, a Cole-Cole plot is presented in Figure 3.8d. In general, the variations in $\varepsilon_{r}{ }^{\prime}, \varepsilon_{r}{ }^{\prime \prime}$ and tan $\delta$ with respect to the frequency are determined by the dipolar molecular relaxations of the polymer chains, filler or any other species in the system, as well as the interfacial polarizations (related to space charge 
relaxations), depending on the frequency range. The dipolar relaxations are mainly predominant at higher frequencies, whereas the interfacial polarizations can be characterized in the low frequency range (usually below $1 \mathrm{~Hz}$ ). Therefore, a broad range of frequencies, $10^{-2}-10^{6} \mathrm{~Hz}$, was applied for the permittivity measurements.

In Figure 3.8a, it is observed that the introduction of untreated silica (NC_0) increases the permittivity at frequencies higher than $1 \mathrm{~Hz}$ compared to the unfilled polymer (UP). This can be a result of the adsorbed moisture on the silica, hydrogen bonded to the silanol groups. A low grafting density of APTES decreases this effect, resulting in a lower permittivity for NC_2 compared to NC_0. However, increasing the APTES grafting density (NC_7) leads to permittivity values even higher than the nanocomposite with unmodified silica. This is due to the presence of the grafted APTES oligomers on the silica, contributing to the dipolar relaxations of the material. A frequency dependent behavior is observed in the real permittivity characteristics, manifested as a steep increase at low frequencies. This increase can be either due to polarization of relatively bigger dipolar species (e.g. contaminations, by-products of filler modification etc.), or occurrence of Maxwell-Wagner or space charge polarizations ${ }^{45}$. Another indicator of space charge polarizations, in addition to the steep increase in $\varepsilon_{r}{ }^{\prime}$, is the emergence of relaxation peaks in $\varepsilon_{r}{ }^{\prime \prime}$ and $\tan \delta$ characteristics of the material ${ }^{13}$. Since these measurements were done at room temperature, no distinct relaxation peak is observed in $\varepsilon_{\mathrm{r}}{ }^{\prime \prime}$ or $\tan \delta$ (Figures $8 \mathrm{~b}$ and $8 \mathrm{c}$ ), as they would be likely to appear at frequencies lower than the measurement range. Nevertheless, an enhancement is observed in the imaginary permittivity and the loss factor at low frequencies. These combined trends of $\varepsilon_{r}{ }^{\prime}, \varepsilon_{r}{ }^{\prime \prime}$ and tan $\delta$, suggest that there can be an effect of DC conductivity and the occurrence of space charge polarization in the studied systems. Interestingly, the slope of these enhancements at low frequencies, tends to be lower for the nanocomposites compared to the base polymer. The enhancement slope in $\varepsilon_{r}^{\prime}, \varepsilon_{r}^{\prime \prime}$ and tan $\delta$ for the nanocomposites tends to decrease further by increasing the APTES grafting density, to the point where only a marginal increase is observed in case of NC_7 with high grafting density. This suggests that the addition of NPs and the introduction of the amine functionality result in either a reduction in the mobility of free polar species in the matrix and/or a suppression in the interfacial polarizations in the nanocomposites. The 
same trend can also be observed in the Cole-Cole plot presented in Figure 3.8d. The lowering of the slope in the low frequency region, in case of the nanocomposites, can be an indication of a lower contribution of DC conductivity in these systems ${ }^{46}$, which is more pronounced in case of nanocomposites with APTES modified silica.
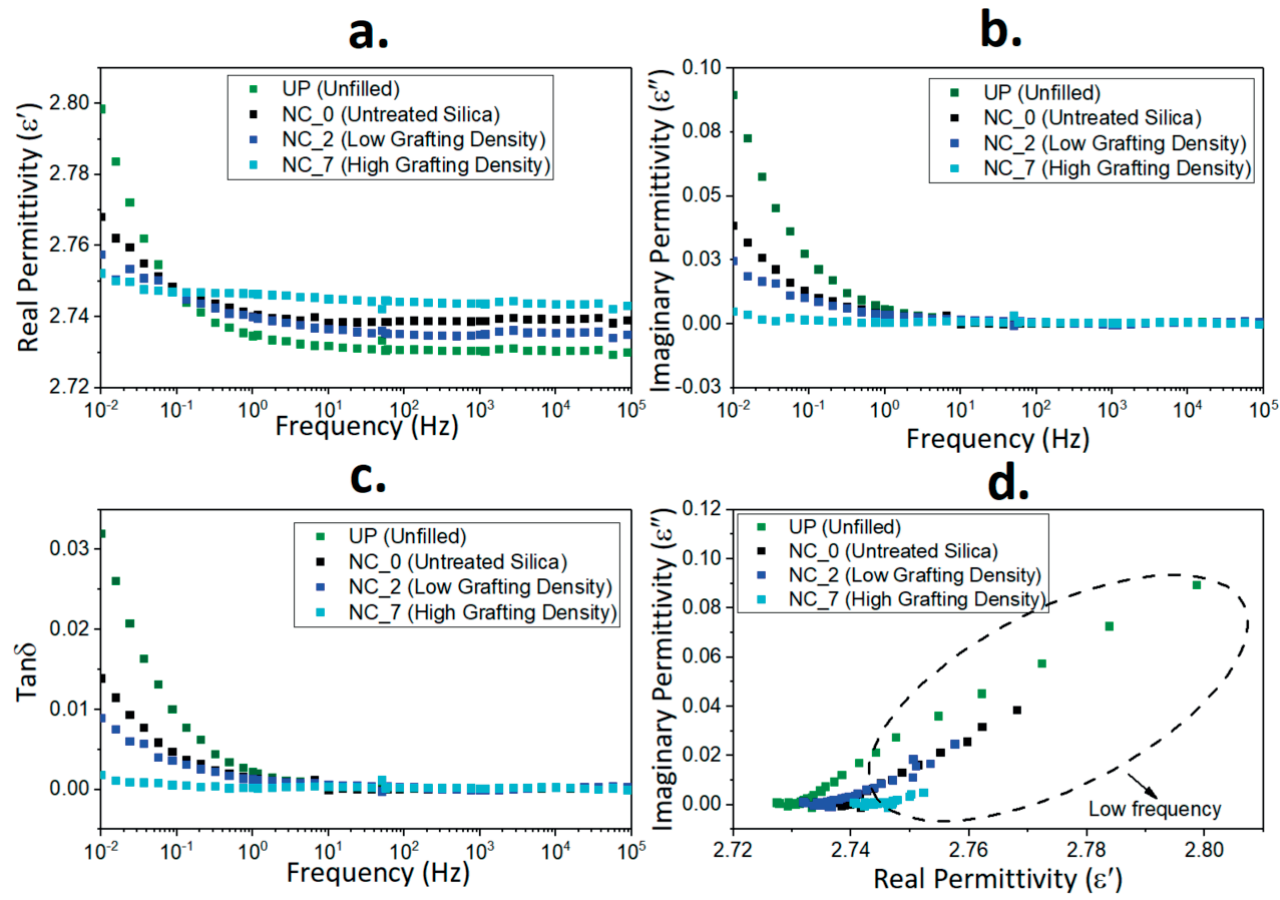

Figure 3.8. a, b, and c: real and imaginary parts of permittivity as well as the loss tangent $(\tan \delta)$ plotted with respect to the frequencies; $d$ : representative Cole-Cole plots for the studied dielectric systems

Figures 3.9a and 3.9b present the TSDC plots for the silica filled NCs as well as of the unfilled polymer (UP) as a reference along with trap level/density distribution calculated using a numerical method presented by Tian et al. ${ }^{47}$. 
a.

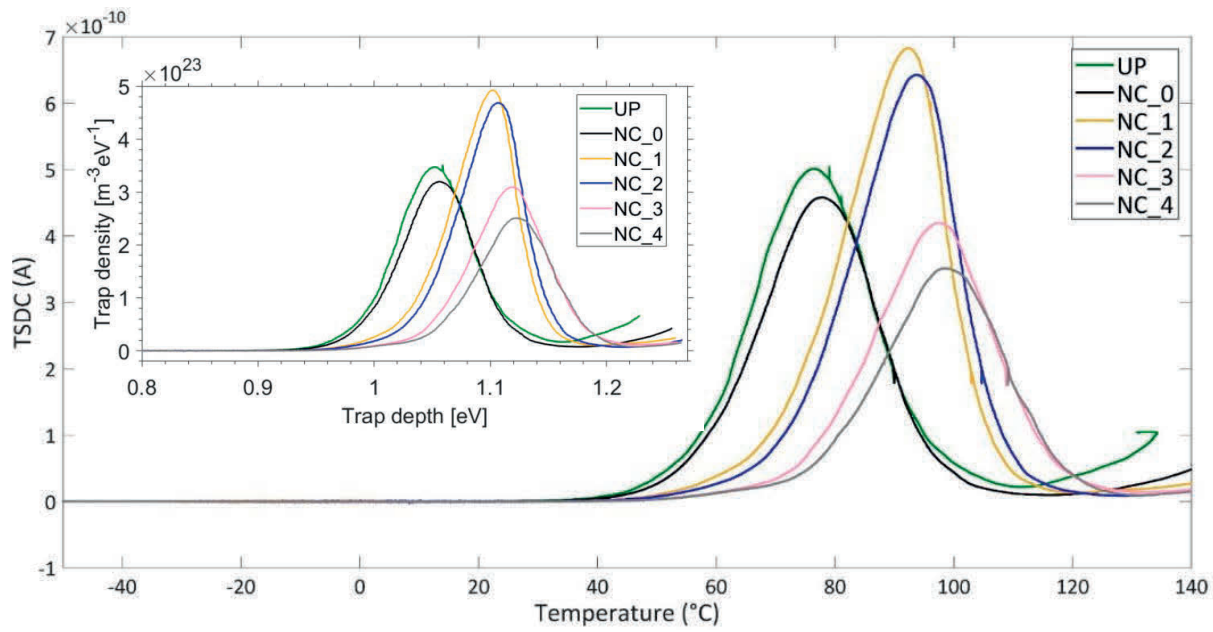

b.

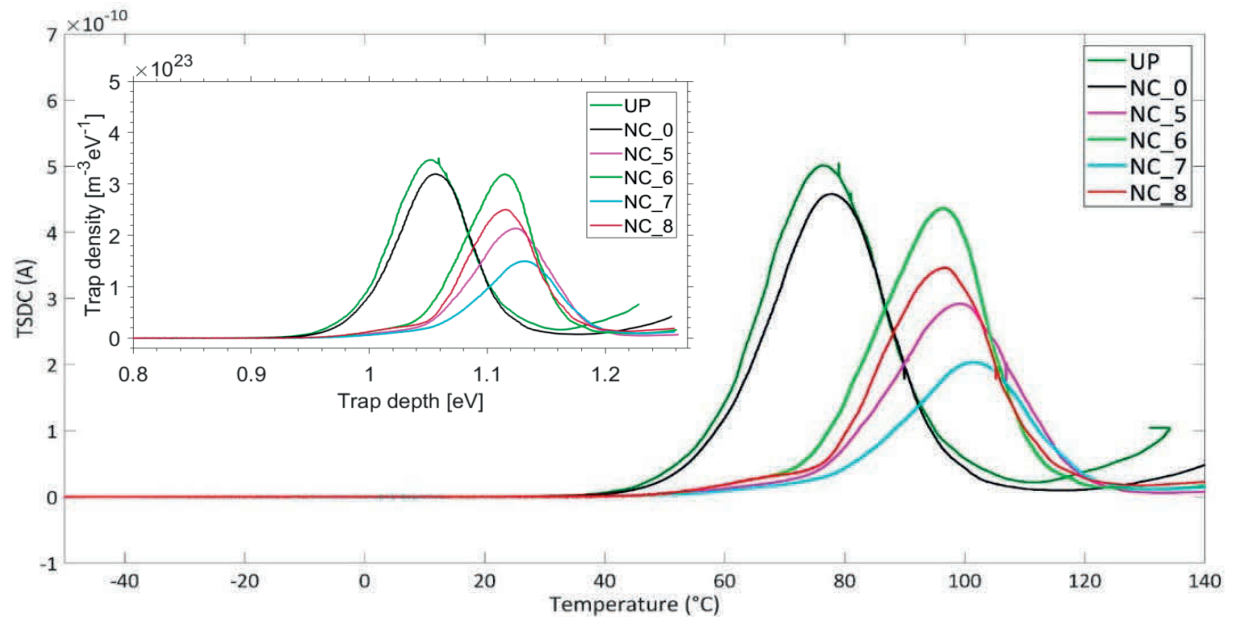


C.

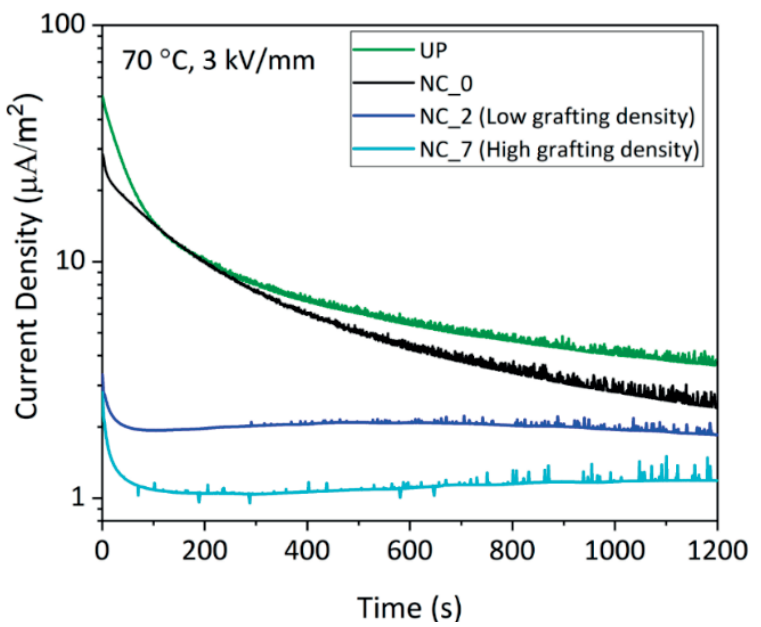

Figure 3.9. a, b: TSDC spectra and MTSDC trap level distribution of injection molded PP/EOC/silica nanocomposites as well as the unfilled reference (UP); c: Isothermal charging current density during the polarization phase

Principally, the TSDC spectra represent the relaxation of the polarized species as well as the accumulated space charge as a result of the electric field. The peaks emerging at temperatures above the glass transition of the semi-crystalline polymers are mainly attributable to the relaxation of the space charge trapped at the interfaces. Upon softening/melting transitions, the trapped charges can be stimulated, and as a result, a peak appears in the current measured by the ammeter. To a first approximation, the temperature and intensity of a TSDC peak can be associated with the depth and density of charge traps, respectively.

The spectra depicted in Figure 3.9 ( $a$ and $b$ ) indicate that by incorporating the treated silica, the TSDC behavior becomes more complex. It is observed that the main TSDC peak shifts towards higher temperatures, indicating that the trap level becomes deeper. In general, in case of samples with the amine functionality on the silica surface, another TSDC peak emerges around $93-97^{\circ} \mathrm{C}$, being indicative of induction of a new deep trap, while a small shoulder is observed around the same temperature $\left(\sim 78^{\circ} \mathrm{C}\right)$ as the main TSDC peaks of the reference samples (UP and NC_0). Comparing NC_2 and NC_7 with the lowest and the highest APTES grafting density on silica, respectively, it can be observed that in case of NC_7, the peak temperature of the TSDC peak is shifted to higher temperatures (deeper 
trap level). This can result in a lower charge mobility in the composites. Moreover, the intensity of the TSDC peak is seen to vary significantly depending on the APTES grafting density, with some of the samples exhibiting reduction in the main TSDC peak intensity; this may be due to (i) the density of the trap states being reduced or (ii) the space charge distribution becoming more uniform in the nanocomposites. Figure 3.9c presents the charging current behavior during the isothermal poling phase for high and low grafting density samples, compared to the references. While it is clear that the polarization step was too short to reach steady-state DC conduction in the reference samples, the transient current densities demonstrate significant differences between the samples. The nanocomposites containing modified NPs show significantly lower current densities, and hence lower apparent conductivity at the end of the poling phase $\left(1.26 \times 10^{-12} \mathrm{~S} / \mathrm{m}, 8 \times 10^{-}\right.$ ${ }^{13} \mathrm{~S} / \mathrm{m}, 6.2 \times 10^{-13} \mathrm{~S} / \mathrm{m}$ and $3.9 \times 10^{-13} \mathrm{~S} / \mathrm{m}$ for UP, NC_0, NC_2 and NC_7, respectively). Accordingly, as it was also suggested by the BDS results, a lower effect of DC conductivity can be another reason for the reduction of the main TSDC peak intensity for the modified samples.

It seems that the apparent depth and density of the traps are not solely influenced by the amount of APTES on the silica surface. This is firstly observed in the samples NC_1 NC_4, which contain silica particles with more or less similar APTES grafting densities. The suppressed TSDC peak for NC_3 and NC_4 can be due to the longer silica modification time, during which condensation of the APTES species is more susceptible. NC_7 and NC_8 also behave differently in terms of TSDC, even though they contain silica samples with similar APTES grafting densities. In case of NC_7, the deep trap TSDC peak intensity is lower compared to NC_8. The same can be observed for samples NC_5 and NC_6. What differs in the above mentioned samples is the time and the temperature of the modification reaction, which affects the morphology and conformity of the deposited APTES layer. A possible explanation for this is that the depth and density of the traps are not only a function of the thickness of the silane layer, but also of its morphology. On the one hand, differences in the deposited APTES layer morphology can solely affect the trap depth and density. On the other hand, changes in the filler surface morphology can affect physical properties of the compounds e.g. crystallization, which can further affect charge trapping behavior of the 
system. Therefore, DSC measurements were performed to analyze and compare melting and crystallization processes in depth.

Melting and crystallization curves for the nanocomposites as well as the unfilled blend are depicted in Figure 3.10. While no noticeable difference is observed in the melting curves (Figure 3.10a), the crystallization curves (Figure 3.10b) show some significant changes upon incorporation of the NPs and changing the modification conditions. Surprisingly, it can be seen that despite the expected nucleating effect of the NPs ${ }^{48}$, the onset of crystallization shifts to a lower temperature after addition of the untreated silica (NC_0). This could be due to adsorption of the polar antioxidants on the silica surface resulting in fewer nucleating sites in the polymer matrix. This also results in less restriction for the crystallites to grow, leading to a higher crystal content compared to UP (see Table 3.2). After the silica surface is treated, the onset of crystallization increases by $4-7^{\circ} \mathrm{C}$ indicating that the deposited APTES layer facilitates nucleation. This increase in the onset of crystallization, however, is not to the same extent for all the samples. Table 3.2 presents the DSC parameters for the nanocomposites NC_5 to NC_8, samples with different APTES layer morphologies. It can be seen that NC_5 and NC_7 exhibit higher crystallization onsets compared to NC_6 and NC_8. This can be explained as follows:

Silica samples 5 and 7 are modified at room temperature, whereas samples 6 and 8 are treated at $80^{\circ} \mathrm{C}$. At lower reaction temperatures, the excess water in the reaction tends to be adsorbed onto the silica surface due to its hydrophilicity. Therefore, the condensation of the APTES and the silanization reaction takes place at the silica surface. This would result in formation of "island-like" structures of APTES on the silica. While the presence of these APTES islands can further contribute to the induction of deep traps ${ }^{49}$, they can act as individual nuclei, inducing interfacial crystallization ${ }^{50-51}$. The broadening that is observed in the NC_5 and NC_7 crystallization curves can also be explained as nucleation and transcrystallization at the silica/polymer interface emerging from the island-like nuclei. While this phenomenon contributes to increasing filler-polymer interactions ${ }^{52}$, the resulting interfacial areas further decrease the space charge injection due to their exposure to the amine functionality. 
At elevated reaction temperatures, however, the water in the system tends to desorb from the silica surface and enters the solution medium ${ }^{53}$. In this situation, the condensation of the silane is more likely to happen before its attachment to the silica. This would result in grafting of longer APTES chains to the silica which leads to a smoother silica surface morphology exhibiting lower nucleation potential. It is also noteworthy that these differences are only observed for the PP phase crystallization curve, suggesting that the NPs are mostly located in this phase rather than in EOC. This observation is also in-line with the SEM images presented in Figure 3.7.

a.
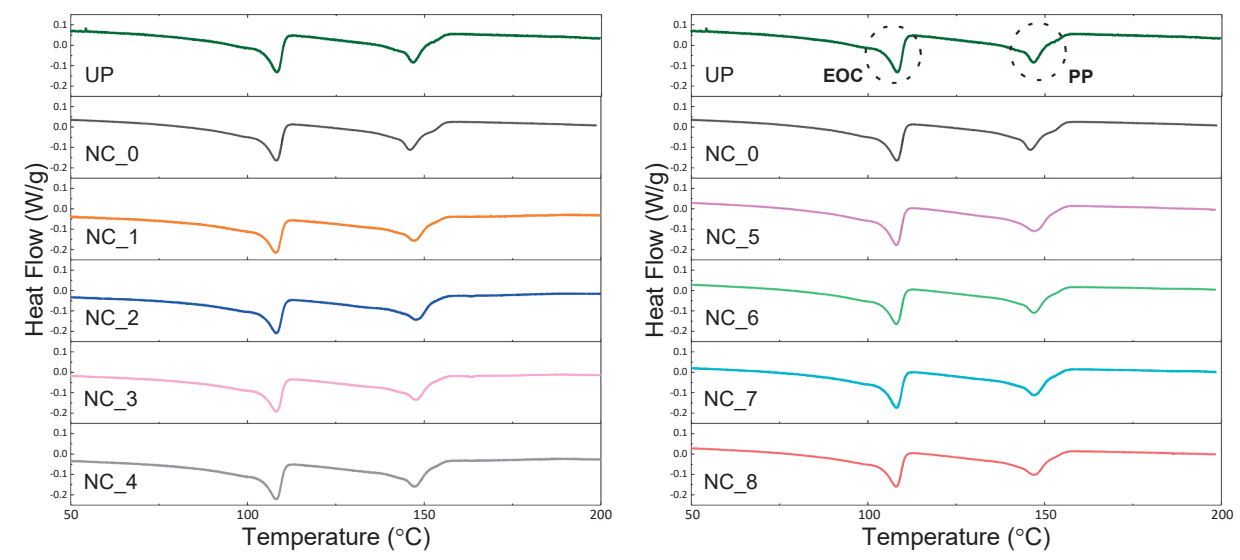

b.
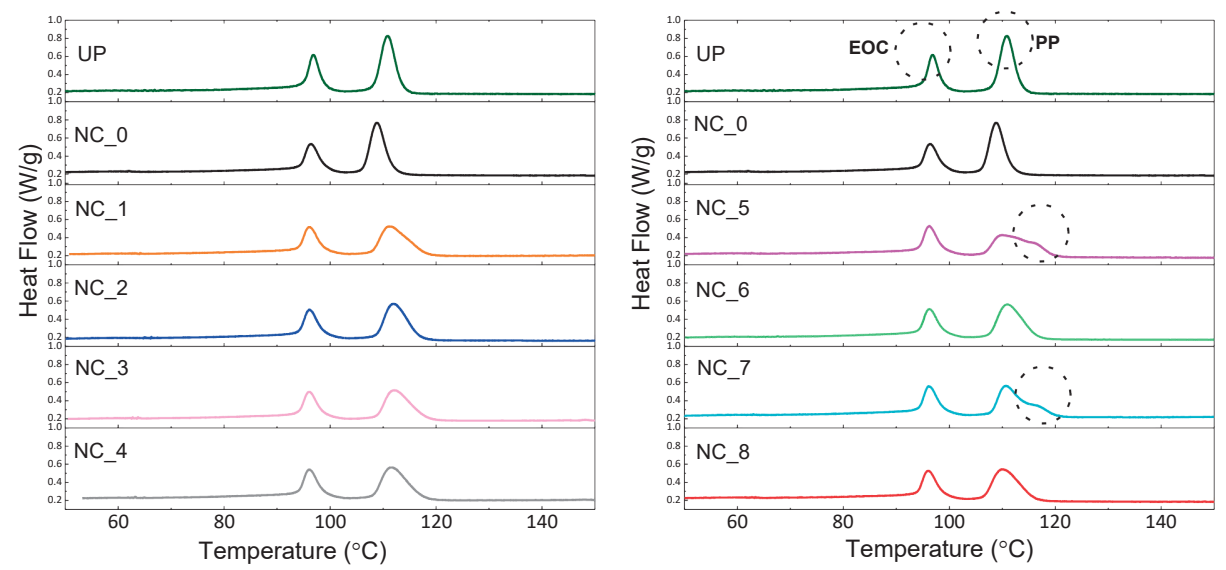

Figure 3.10. DSC measurements; a: melting b: crystallization curves of the nanocomposites 
Table 3.2. DSC parameters of selected nanocomposites

\begin{tabular}{|c|c|c|c|c|c|c|}
\hline Sample & $\begin{array}{c}T_{m} \text { Onset } \\
\left({ }^{\circ} \mathrm{C}\right)\end{array}$ & $\begin{array}{c}\mathrm{T}_{\mathrm{c}} \text { Onset } \\
\left({ }^{\circ} \mathrm{C}\right)\end{array}$ & $\begin{array}{l}P P T_{C} \\
\left({ }^{\circ} \mathrm{C}\right)\end{array}$ & $\begin{array}{c}\mathrm{EOC}_{\mathrm{C}} \\
\left({ }^{\circ} \mathrm{C}\right)\end{array}$ & $\begin{array}{l}\text { Enthalpy of } \\
\text { Melting }(\mathrm{J} / \mathrm{g})\end{array}$ & $\begin{array}{c}\% \\
\text { Crystallinity }\end{array}$ \\
\hline UP & 102.5 & 115 & 110.9 & 96.9 & 87.4 & 35 \\
\hline NC_O & 102.1 & 112.2 & 108.7 & 96.7 & 97.8 & 39 \\
\hline NC_5 & 100.5 & 119.7 & 110.1 & 96.5 & 89.9 & 36 \\
\hline NC_6 & 101.4 & 117.5 & 111 & 96.3 & 79.3 & 31 \\
\hline NC_7 & 101.3 & 120.3 & 110.8 & 96.1 & 91.4 & 36 \\
\hline NC_8 & 101.7 & 116.5 & 110.1 & 95.9 & 87.3 & 35 \\
\hline
\end{tabular}

\section{Conclusions}

Successful deposition of APTES on fumed silica nanoparticles was confirmed by TGA, FTIR and XPS test results. Different modification conditions resulted in different levels of grafting density as well as different APTES film morphologies. From TSDC data, a substantial shift to higher energy levels and a significant reduction in intensity was observed for the space charge relaxation peak. These observations, in conjunction with BDS results, suggest that the presence of the amine moiety at the filler-polymer interface induces deep trap states. In this case, the deep trapped charges hinder further injection of space charges. The space charge injection, however, is not only affected by the amount of deposited silane, but also by its morphology. It was suggested that upon deposition of APTES on silica, an "islandlike" morphology can be formed on the surface at lower modification temperatures when water is present in the system. These "islands" can facilitate nucleation by acting as individual nuclei and inducing transcrystallization at the filler-polymer interface. Moreover, the presence of "island-like" structures on the silica surface would further contribute to the induction of deep traps at the filler-polymer interface which results in reduction of space charge injection. 


\section{References}

[1] Ma, D.; Treese, A. H.; Siegel, R.; Anna, C.; Eva, M.; Carina, Ö.; Linda, S. S., Influence of nanoparticle surface modification on the electrical behaviour of polyethylene nanocomposites. Nanotechnology, 16 (6), 2005, p 724.

[2] Huang, X.; Jiang, P.; Yin, Y., Nanoparticle surface modification induced space charge suppression in linear low density polyethylene. Applied Physics Letters, 95 (24), 2009, p 242905.

[3] Huang, X.; Zheng, Y.; Jiang, P.; Yin, Y., Influence of nanoparticle surface treatment on the electrical properties of cycloaliphatic epoxy nanocomposites. IEEE Transactions on Dielectrics and Electrical Insulation, 17 (2), 2010, pp 635-643.

[4] Montanari, G. C.; Seri, P.; Paajanen, M.; Lahti, K.; Rytöluoto, I.; Ritamäki, M.; Blume, A.; Dierkes, W.; Mahtabani, A.; He, X. Potentiality of nanofilled thermoplastic insulation for DC cables and capacitors, 2018 IEEE 2nd International Conference on Dielectrics (ICD), 2018; pp 1-4.

[5] Tian, F.; Yao, J.; Li, P.; Wang, Y.; Wu, M.; Lei, Q., Stepwise electric field induced charging current and its correlation with space charge formation in LDPE/ZnO nanocomposite. IEEE Transactions on Dielectrics and Electrical Insulation, 22 (2), 2015, pp 1232-1239.

[6] Zhou, Y.; Hu, J.; Dang, B.; He, J., Titanium oxide nanoparticle increases shallow traps to suppress space charge accumulation in polypropylene dielectrics. RSC Advances, 6 (54), 2016, pp 48720-48727.

[7] Zhou, Y.; He, J.; Hu, J.; Dang, B., Surface-modified MgO nanoparticle enhances the mechanical and direct-current electrical characteristics of polypropylene/polyolefin elastomer nanodielectrics. Journal of Applied Polymer Science, 133 (1), 2016.

[8] Zhang, L.; Khani, M. M.; Krentz, T. M.; Huang, Y.; Zhou, Y.; Benicewicz, B. C.; Nelson, J. K.; Schadler, L. S., Suppression of space charge in crosslinked polyethylene filled with poly(stearyl methacrylate)-grafted SiO2 nanoparticles. Applied Physics Letters, 110 (13), 2017, p 132903.

[9] Smith, R. C.; Liang, C.; Landry, M.; Nelson, J. K.; Schadler, L. S., The mechanisms leading to the useful electrical properties of polymer nanodielectrics. IEEE Transactions on Dielectrics and Electrical Insulation, 15 (1), 2008, pp 187-196.

[10] Lewis, T. J., Nanometric dielectrics. IEEE Transactions on Dielectrics and Electrical Insulation, 1 (5), 1994, pp 812-825.

[11] Fabiani, D.; Montanari, G. C.; Testa, L.; Schifani, R.; Guastavino, F.; Bellucci, F.; Deorsola, F. Effect of water adsorption on the dielectric properties of polymer nanocomposites, 2008 International Symposium on Electrical Insulating Materials (ISEIM 2008), 2008; pp 510-513.

[12] Montanari, G.; Cavallini, A.; Guastavino, F.; Coletti, G.; Schifani, R.; del Casale, M. d. L.; Camino, G.; Deorsola, F. Microscopic and nanoscopic EVA composite investigation: electrical properties and effect of purification treatment, The 17th Annual Meeting of the IEEE Lasers and Electro-Optics Society, 2004. LEOS 2004., IEEE: 2004; pp 318-321.

[13] Nelson, J. K., Dielectric polymer nanocomposites Springer: 2010;

[14] Xiao, Z.; Li, Y.; Ma, D.; Schadler, L. S.; Akpalu, Y. A., Probing the use of small-angle light scattering for characterizing structure of titanium dioxide/low-density polyethylene 
nanocomposites. Journal of Polymer Science Part B: Polymer Physics, 44 (7), 2006, pp 10841095.

[15] Brown, D.; Mélé, P.; Marceau, S.; Albérola, N. D., A Molecular Dynamics Study of a Model Nanoparticle Embedded in a Polymer Matrix. Macromolecules, 36 (4), 2003, pp 13951406.

[16] Sternstein, S. S.; Zhu, A.-J., Reinforcement Mechanism of Nanofilled Polymer Melts As Elucidated by Nonlinear Viscoelastic Behavior. Macromolecules, 35 (19), 2002, pp $7262-$ 7273.

[17] Mahtabani, A.; Alimardani, M.; Razzaghi-Kashani, M., Further evidence of fillerfiller mechanical engagement in rubber compounds filled with silica treated by long-chain silane. Rubber Chemistry and Technology, 90 (3), 2017, pp 508-520.

[18] He, X.; Rytöluoto, I.; Anyszka, R.; Mahtabani, A.; Saarimäki, E.; Lahti, K.; Paajanen, M.; Dierkes, W.; Blume, A., Surface Modification of Fumed Silica by Plasma Polymerization of Acetylene for PP/POE Blends Dielectric Nanocomposites. Polymers, 11 (12), 2019, p 1957. [19] Kasperovich, V.; Wong, K.; Tikhonov, G.; Kresin, V. V., Electron Capture by the Image Charge of a Metal Nanoparticle. Physical Review Letters, 85 (13), 2000, pp 2729-2732. [20] Khalil, M. S., The role of BaTiO/sub 3/ in modifying the dc breakdown strength of LDPE. IEEE Transactions on Dielectrics and Electrical Insulation, 7 (2), 2000, pp 261-268.

[21] Alimardani, M.; Razzaghi-Kashani, M.; Karimi, R.; Mahtabani, A., Contribution Of Mechanical Engagement And Energetic Interaction In Reinforcement Of SBR-Silane Treated Silica Composites. Rubber Chemistry and Technology, 89 (2), 2016, pp 292-305.

[22] Natarajan, B.; Li, Y.; Deng, H.; Brinson, L. C.; Schadler, L. S., Effect of Interfacial Energetics on Dispersion and Glass Transition Temperature in Polymer Nanocomposites. Macromolecules, 46 (7), 2013, pp 2833-2841.

[23] Mathabani, A.; Rytöluoto, I.; He, X.; Saarimäki, E.; Lahti, K.; Paajanen, M.; Anyszka, R.; Dierkes, W.; Blume, A. Solution Modified Fumed Silica and Its Effect on Charge Trapping Behavior of PP/POE/Silica Nanodielectrics, Proceedings of the Nordic Insulation Symposium, 2019; pp 129-133.

[24] He, X.; Mahtabani, A.; Rytöluoto, I.; Saarimäki, E.; Lahti, K.; Paajanen, M.; Anyszka, R.; Dierkes, W.; Blume, A. Surface Modification of Fumed Silica by Dry Silanization for PPbased Dielectric Nanocomposites, 2019 2nd International Conference on Electrical Materials and Power Equipment (ICEMPE), IEEE: 2019; pp 254-259.

[25] Siddabattuni, S.; Schuman, T. P.; Dogan, F., Dielectric Properties of PolymerParticle Nanocomposites Influenced by Electronic Nature of Filler Surfaces. ACS Applied Materials \& Interfaces, 5 (6), 2013, pp 1917-1927.

[26] Dongling, M.; Treese, A. H.; Richard, W. S.; Anna, C.; Eva, M.; Carina, Ö.; Linda, S. S., Influence of nanoparticle surface modification on the electrical behaviour of polyethylene nanocomposites. Nanotechnology, 16 (6), 2005, p 724.

[27] Lau, K. Y.; Vaughan, A. S.; Chen, G.; Hosier, I. L. Space charge dynamics in silicabased polyethylene nanocomposites, 2013 IEEE International Conference on Solid Dielectrics (ICSD), 2013; pp 880-883.

[28] Yamano, Y.; Endoh, H., Increase in breakdown strength of PE film by additives of azocompounds. IEEE Transactions on Dielectrics and Electrical Insulation, 5 (2), 1998, pp 270-275. 
[29] Huang, Y.; Wu, K.; Bell, M.; Oakes, A.; Ratcliff, T.; Lanzillo, N. A.; Breneman, C.; Benicewicz, B. C.; Schadler, L. S., The effects of nanoparticles and organic additives with controlled dispersion on dielectric properties of polymers: Charge trapping and impact excitation. Journal of Applied Physics, 120 (5), 2016, p 055102.

[30] Huang, Y.; Schadler, L. S., On the nature of high field charge transport in reinforced silicone dielectrics: Experiment and simulation. Journal of Applied Physics, 120 (5), 2016, p 055101.

[31] Zhang, C.; Mizutani, T.; Kaneko, K.; Mori, T.; Ishioka, M., Space charge behaviors of low-density polyethylene blended with polypropylene copolymer. Polymer, 43 (8), 2002, pp 2261-2266.

[32] Wang, X.; Lv, Z.; Wu, K.; Chen, X.; Tu, D.; Dissado, L. A., Study of the factors that suppress space charge accumulation in LDPE nanocomposites. IEEE Transactions on Dielectrics and Electrical Insulation, 21 (4), 2014, pp 1670-1679.

[33] Xu, X.; Li, B.; Lu, H.; Zhang, Z.; Wang, H., The effect of the interface structure of different surface-modified nano-SiO2 on the mechanical properties of nylon 66 composites. Journal of Applied Polymer Science, 107 (3), 2008, pp 2007-2014.

[34] Fukuyama, Y.; Kawai, T.; Kuroda, S.-i.; Toyonaga, M.; Taniike, T.; Terano, M., The effect of the addition of polypropylene grafted $\mathrm{SiO} 2$ nanoparticle on the crystallization behavior of isotactic polypropylene. Journal of Thermal Analysis and Calorimetry, 113 (3), 2013, pp 1511-1519.

[35] Dang, B.; He, J.; Hu, J.; Zhou, Y., Large improvement in trap level and space charge distribution of polypropylene by enhancing the crystalline - amorphous interface effect in blends. Polymer International, 65 (4), 2016, pp 371-379.

[36] Chi, X.; Cheng, L.; Liu, W.; Zhang, X.; Li, S., Characterization of Polypropylene Modified by Blending Elastomer and Nano-Silica. Materials, 11 (8), 2018, p 1321.

[37] Pasternack, R. M.; Rivillon Amy, S.; Chabal, Y. J., Attachment of 3(Aminopropyl)triethoxysilane on Silicon Oxide Surfaces: Dependence on Solution Temperature. Langmuir, 24 (22), 2008, pp 12963-12971.

[38] Qiao, B.; Wang, T.-J.; Gao, H.; Jin, Y., High density silanization of nano-silica particles using $\gamma$-aminopropyltriethoxysilane (APTES). Applied Surface Science, 351, 2015, pp 646-654.

[39] Ignacio, A.-C.; Verena, K.; Curtis, R.; Kevin W, E.; Johannes, S.; Cardona, A.; Seung, H. S., Trainable Weka Segmentation: a machine learning tool for microscopy pixel classification. Bioinformatics, 33 (15), 2017, pp 2424-2426.

[40] Blume, A.; Jin, J.; Mahtabani, A.; He, X.; Kim, S.; Andrzejewska, Z. New Structure Proposal for Silane Modified Silica, International Rubber Conference, IRC 2019, London, 2019.

[41] Vansant, E. F.; Van Der Voort, P.; Vrancken, K. C., Characterization and chemical modification of the silica surface Elsevier: 1995; Vol. 93.

[42] Zhong, G. J.; Li, Z. M., Injection molding-induced morphology of thermoplastic polymer blends. Polymer Engineering \& Science, 45 (12), 2005, pp 1655-1665.

[43] Ying, J.; Xie, X.; Peng, S.; Zhou, H.; Li, D., Morphology and rheology of PP/POE blends in high shear stress field. Journal of Thermoplastic Composite Materials, 31 (9), 2017, pp 1263-1280. 
[44] Andritsch, T.; Vaughan, A.; Stevens, G. C., Novel insulation materials for high voltage cable systems. IEEE Electrical Insulation Magazine, 33 (4), 2017, pp 27-33.

[45] Nelson, J. K.; Fothergill, J. C., Internal charge behaviour of nanocomposites. Nanotechnology, 15 (5), 2004, p 586.

[46] Kao, K. C., Dielectric phenomena in solids Elsevier: 2004;

[47] Tian, F.; Bu, W.; Shi, L.; Yang, C.; Wang, Y.; Lei, Q., Theory of modified thermally stimulated current and direct determination of trap level distribution. Journal of Electrostatics, 69 (1), 2011, pp 7-10.

[48] Abbasi-Shirsavar, M.; Baghani, M.; Taghavimehr, M.; Golzar, M.; Nikzad, M.; Ansari, M.; George, D., An experimental-numerical study on shape memory behavior of $\mathrm{PU} / \mathrm{PCL} / \mathrm{ZnO}$ ternary blend. Journal of Intelligent Material Systems and Structures, 30 (1), 2018, pp 116-126.

[49] Rychkov, A. A.; Trifonov, S. A.; Kuznetsov, A. E.; Sosnov, E. A.; Rychkov, D. A.; Malygin, A. A., Influence of chemical modification of the surface of low-density polyethylene on its electret properties. Russian Journal of Applied Chemistry, 80 (3), 2007, pp 461-465.

[50] Lin, C. W.; Ding, S. Y.; Hwang, Y. W., Interfacial crystallization of isotactic polypropylene molded against the copper surface with various surface roughnesses prepared by an electrochemical process. Journal of Materials Science, 36 (20), 2001, pp 4943-4948.

[51] Abdou, J. P.; Reynolds, K. J.; Pfau, M. R.; van Staden, J.; Braggin, G. A.; Tajaddod, N.; Minus, M.; Reguero, V.; Vilatela, J. J.; Zhang, S., Interfacial crystallization of isotactic polypropylene surrounding macroscopic carbon nanotube and graphene fibers. Polymer, 91, 2016, pp 136-145.

[52] Ning, N.; Fu, S.; Zhang, W.; Chen, F.; Wang, K.; Deng, H.; Zhang, Q.; Fu, Q., Realizing the enhancement of interfacial interaction in semicrystalline polymer/filler composites via interfacial crystallization. Progress in Polymer Science, 37 (10), 2012, pp 1425-1455.

[53] Howarter, J. A.; Youngblood, J. P., Optimization of Silica Silanization by 3Aminopropyltriethoxysilane. Langmuir, 22 (26), 2006, pp 11142-11147. 


\section{CHAPTER 4}

Ureido and Methacrylate Functionalized Silica Nanoparticles in PolypropyleneBased Nanodielectrics 


\begin{abstract}
$^{1}$
Surface modification of nanoparticles is a promising way to tailor the interfacial properties in nanocomposites. In case of dielectric nanocomposites, introducing different functional groups to the nanoparticles' surface may induce localized states (traps) that can enhance the dielectric performance of the material depending on their density and energy levels. Furthermore, surface modification of the filler can affect the morphology and crystallization of the nanocomposites which can also alter the dielectric response of the material. In this study, we firstly demonstrate the successful functionalization of silica nanoparticles using 3-(trimethoxysilyl)propyl methacrylate (TMPM) and 1-[3(trimethoxysilyl)propyl]urea (TMPU) as modifying agents. Subsequently, the effect of such modifications on the crystallization behavior and dispersion quality of the nanoparticles is studied in nanocomposites based on polypropylene (PP)/ethylene-octene-copolymer (EOC) blends at $1 \%$ and $5 \%$ of filler concentrations. The studied nanocomposites are then subjected to dielectric measurements. Broadband dielectric spectroscopy (BDS) and thermally stimulated depolarization current (TSDC) tests are performed to analyze the alterations in charge trapping and transport as well as space charge formation in the aforementioned systems, upon introducing methacrylate and ureido functional groups to the filler-polymer interfaces. Our results show that both ureido and methacrylate functional groups introduce localized states, but with different energy levels. Nitrogen containing ureido groups in TMPU tend to induce deeper traps to the filler-polymer interfaces, compared to the methacrylate silane modification. This resulted in a suppression of space charge formation at the interfaces under a high electric field.
\end{abstract}

1 The results of this chapter are published in IEEE Access, vol. 9, pp. 130340-130352, 2021. (10.1109/ACCESS.2021.3112849) 


\section{Introduction}

In the previous chapter, it was shown that by grafting amino-functional groups to the surface of silica nanoparticles (NPs), deep traps are introduced to the filler-polymer interfaces. These deep traps, when occupied, could suppress the formation of space charge in the nanocomposites (NCs) under a DC electric field. These observations imply that the presence of nitrogen-containing functional groups at the surface of NPs can induce such effect. Therefore, in order to further examine the above hypothesis, it is of interest to compare its effect with that of another type of modification. Yet, nitrogen-containing functional groups can be detrimental to the dispersion quality of the NPs in the polymer matrix due to their strong tendency to form hydrogen bonds with the surrounding silanol groups on silica ${ }^{1}$.

The addition of NPs results in electrical defects in the dielectric NCs ${ }^{2}$, therefore, improving their dispersion in the polymer matrix can lead to improvements of the dielectric properties such as breakdown strength ${ }^{3}$. Several studies have reported the significance of filler dispersion in determining the properties of nanodielectrics ${ }^{4-7}$. Surface treatment of nanofillers is a promising way to improve dispersion qualities in NCs, and benefit from their large interfacial areas ${ }^{8}$. Accordingly, NPs dispersion can be influenced by the type of functional groups in the structure of the modifying agent. Moreover, altering the chemistry of the NPs surface can affect the morphological properties of the NCs, particularly in case of semi-crystalline polymers ${ }^{9-10}$. Therefore, studying the morphological aspects of nanodielectrics is of great importance for tailoring their macroscopic dielectric properties.

It has been shown that tailoring the chemical composition of the NPs surface can result in improvement of the dielectric properties of the NCs, not only by influencing their morphological aspects, but also by introducing electrically active functional groups to the filler-polymer interfaces ${ }^{3,11}$. Filler functionalization can introduce new localized states into the nanodielectric, and consequently affect the charge trapping and transport as well as space charge phenomena in the system. 
In this chapter, we firstly demonstrate the successful functionalization of silica NPs using 3-(trimethoxysilyl)propyl methacrylate (TMPM) and 1-[3-(trimethoxysilyl)propyl]urea (TMPU) as modifying agents (see Figure 4.1), with the latter having a nitrogen-containing ureido group in its structure. Subsequently, the effect of such modifications on the crystallization behavior and dispersion quality of the NPs is studied in NCs based on polypropylene (PP)/ethylene-octene-copolymer (EOC) blends. The studied NCs are then subjected to dielectric measurements. Broadband dielectric spectroscopy (BDS) and thermally stimulated depolarization current (TSDC) tests are performed to analyze the alterations in charge trapping and transport as well as space charge formation in the aforementioned NCs, upon introducing methacrylate and ureido functional groups to the filler-polymer interfaces. Since the effective grafting density of the silanes is limited by their reactivity and the specific surface area of the NPs, higher concentrations of these functional groups in the system are only possible by increasing the filler content. Therefore, two different filler concentrations are studied for the modified samples, in order to further investigate the effect of these functional groups on the dielectric properties of the NCs.

a.<smiles>C=C(C)C(=O)OCCC[Si](C)(OC)OC</smiles><smiles>CO[Si](C)(CCCNC(N)=O)OC</smiles>

Figure 4.1. Chemical structures of a: 3-(trimethoxysilyl)propyl methacrylate (TMPM), and b: 1-[3(trimethoxysilyl)propyl]urea (TMPU).

\section{Experimental Work}

\subsection{Silica Modification}

AEROSIL 200 silica NPs were provided by Evonik Industries, Germany. The silica NPs were treated in liquid phase in a glass round bottom flask immersed in an oil heating bath equipped with a mechanical stirrer, cooler and thermometer. 3-(trimethoxysilyl)propyl methacrylate (TMPM) and 1-[3-(trimethoxysilyl)propyl]urea (TMPU) were purchased from 
Sigma-Aldrich, Germany, and used as the modifying agent without further purification. Toluene (Boom Chemicals, the Netherlands) was used as the reaction medium, and a stoichiometric amount of water was added to the reactor to hydrolize the added amount of silane. The modification reaction took place at $80^{\circ} \mathrm{C}$ for $24 \mathrm{~h}$. The silica NPs were stored in an oven at $120^{\circ} \mathrm{C}$ overnight prior to modification, and cleaned after modification via Soxhlet extraction using ethanol as the solvent. The latter was done in order to remove any unreacted precursor and reaction by-product from the silica samples.

\subsection{Silica Characterization}

Thermogravimetric Analysis (TGA) and Diffuse Reflectance Fourier Transform Infrared Spectroscopy (DRIFTS) were performed according to what was described in Chapter 3.

\subsection{Preparation of the Nanocomposites}

Nanocomposites based on polypropylene/ethylene-octene copolymer (PP/EOC) blends were prepared by the addition $1 \mathrm{w} \%$ and $5 \mathrm{w} \%$ of the silica NPs as well as an antioxidant package $(0.3 \mathrm{w} \%)$ to the polymer matrix using equipment described in Chapter 3.

\subsection{Morphology and Crystallinity Analysis}

Scanning Electron Microscopy (SEM) was performed on the dielectric NCs using a Jeol JSM-6400 (Japan) to study the NP dispersion and polymer blend morphology. Sample preparation was done both with and without gold sputtering in order to visualize the particles and the polymer phases in more detail. The silica cluster size distribution in the studied NCs was analyzed using open-source ImageJ software with Trainable Weka Segmentation plugin ${ }^{12}$. Differential Scanning Calorimetry (DSC) was utilized according to the description in Chapter 3.

\subsection{Thermally Stimulated Depolarization Current (TSDC)}

Thermally Stimulated Depolarization Current (TSDC) measurements were performed in accordance with the description in Chapter 3. 


\subsection{Broadband Dielectric Spectroscopy (BDS)}

These measurements were performed according to what was described in Chapter 3.

\section{Results and Discussions}

Figure 4.2 presents the TGA thermograms of both modified samples along with the untreated silica. The $1.5 \%$ mass loss of the untreated silica is due to the release of physisorbed and chemisorbed water. The $\sim 1.2 \%$ of mass loss occurring above $150{ }^{\circ} \mathrm{C}$ can be attributed to the siloxanation of silanol groups on the silica surface, and accounts for a density of $4 \mathrm{SiOH} / \mathrm{nm}^{213}$. Regarding the modified silica, one can calculate the silane grafting density from the TGA mass loss according to Equation 4-1.

$$
\text { Silane Grafting Density }\left(\frac{\mathrm{mmol}}{\mathrm{g} \text { silica }}\right)=\frac{\Delta W \times 1000}{M_{w} \times(100-\Delta W)}
$$

Where $\Delta W$ is the main mass loss of the thermogram and $M_{w}$ is the molecular weight of the silane in the temperature ranges of $270{ }^{\circ} \mathrm{C}$ to $850{ }^{\circ} \mathrm{C}$, and $200{ }^{\circ} \mathrm{C}$ to $850{ }^{\circ} \mathrm{C}$ for the TMPM and TMPU-modified silica, respectively.

TMPM and TMPU-modified silicas exhibit mass losses of $5.8 \%$ and $15 \%$, which account for grafting densities of $0.25 \frac{\mathrm{mmol}}{\mathrm{g} \mathrm{silica}}$ and $0.79 \frac{\mathrm{mmol}}{\mathrm{g} \mathrm{silica}}$, respectively. Due to the hydrogen bond interactions between both silanes and the silanol groups on the silica surface, both reactions are expected to proceed in a self-catalyzing manner ${ }^{14}$. The Si-O-H $\cdots \mathrm{N}$ hydrogen bonds in the case of TMPU, however, are generally stronger than those of the $\mathrm{Si}-\mathrm{O}-\mathrm{H} \cdots \mathrm{O}$ for TMPM, due to the higher electronegativity of nitrogen ${ }^{15}$. This may be a reason for the higher grafting density of TMPU on silica compared to that of TMPM. Furthermore, the stronger hydrogen bond interactions between the silane molecules in TMPU may lead to oligomerization of this silane and formation of longer chains. This can manifest itself as 
multiple steps in the TGA thermograms. The second mass loss step in case of TMPUmodified silica starting from $\sim 300{ }^{\circ} \mathrm{C}$ may be due to this reason.

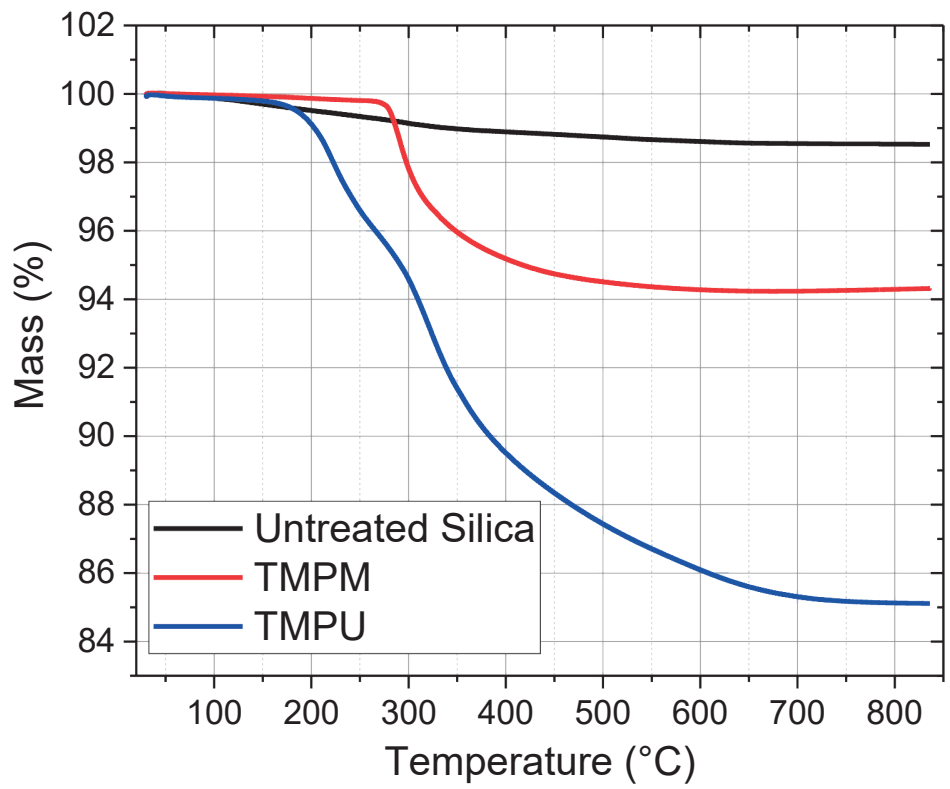

Figure 4.2. TGA thermograms of the unmodified and modified silica NPs. The main mass loss step is related to the release of the grafted precursor

The DRIFTS spectra in Figure 4.3 represent the chemistry of the surface before and after modification, and confirm the successful deposition of each silane onto the nanoparticles. It is clear that the band at $3750 \mathrm{~cm}^{-1}$ corresponding to the isolated silanol groups disappears upon modification of the silica. This is indicative of effective coverage of nanoparticles upon deposition of the silane. The TMPM-modified silica exhibits the characteristic bands of methacrylate groups, $C=C$ and $C=O$ at $1640 \mathrm{~cm}^{-1}$ and $1690 \mathrm{~cm}^{-1}$, respectively ${ }^{16}$. The $\mathrm{C}-\mathrm{H}$ stretching vibration bands, from $2800 \mathrm{~cm}^{-1}$ to $3000 \mathrm{~cm}^{-1}$, appear in both TMPM and TMPU spectra, which are mostly attributable to the propyl spacer chain in both silanes. The TMPU spectrum contains the characteristic bands related to the ureido groups, $\mathrm{C}=\mathrm{N}, \mathrm{C}=\mathrm{O}$ and $\mathrm{N}-\mathrm{H}$, appearing at $1560 \mathrm{~cm}^{-1}, 1690 \mathrm{~cm}^{-1}$ and $3350 \mathrm{~cm}^{-1}$, respectively ${ }^{17}$. 


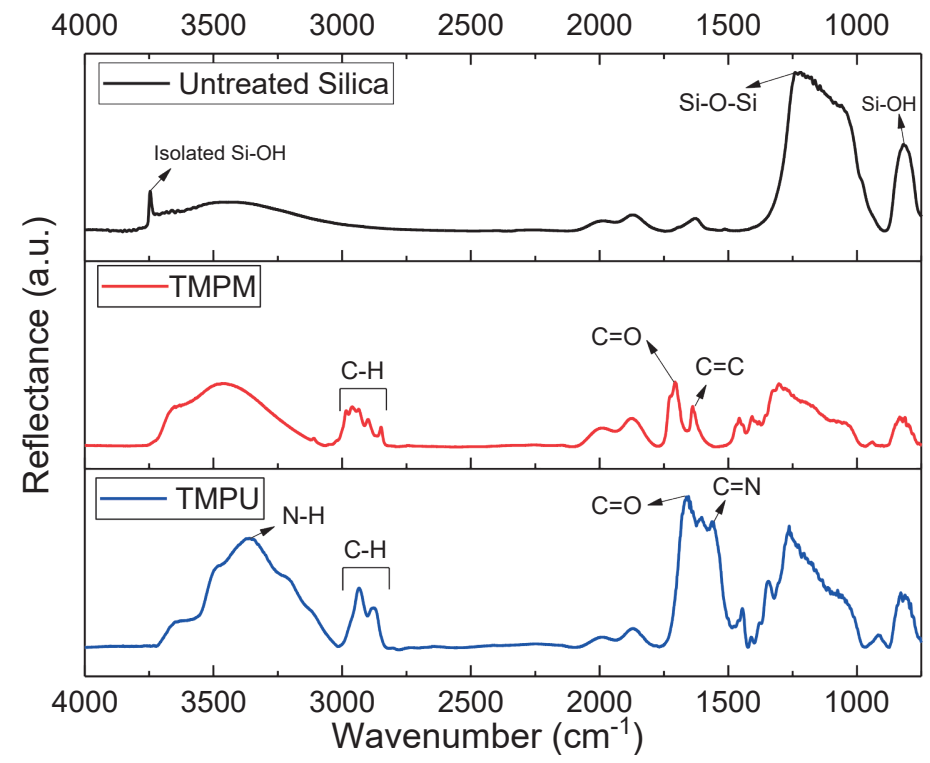

Figure 4.3. DRIFTS spectra for the modified and unmodified NPS

In order to analyze the filler dispersion in the NCs, SEM images along with histograms of particle size distributions are presented in Figure 4.4. The mean silica cluster size for untreated silica in the PP/EOC matrix (Sample 0) is approximately $300 \mathrm{~nm}$. In case of $1 \%$ silica concentration, a mean cluster size around $160 \mathrm{~nm}$ is achieved with the TMPM modification, indicating a relatively better NPs dispersion with this type of treatment. Whereas, TMPU-modified silica exhibits a mean cluster size of $300 \mathrm{~nm}$. This can be due to the nitrogen containing ureido groups deposited on the NPs surface interacting strongly via hydrogen bonds resulting in relatively larger clusters compared to TMPM. Another reason can be the oligomerization of the ureido silane, binding the particles by strong covalent bonds. The much higher grafting density of TMPU shown in Figure 4.2 is an evidence for this oligomerization. Increasing the filler content to $5 \%$ results in relatively bigger clusters for both types of modification as TMPM_5 and TMPU_5 exhibit mean aggregate sizes around $200 \mathrm{~nm}$ and $400 \mathrm{~nm}$, respectively. The polymer blend exhibits a well dispersed two-phase morphology, with the EOC domains elongated along the direction of the flow in the mold (see TMPM_1 and TMPU_5). This is not visible in the TMPM_5 and TMPU_5 images since these samples were gold sputtered to achieve higher resolutions. 

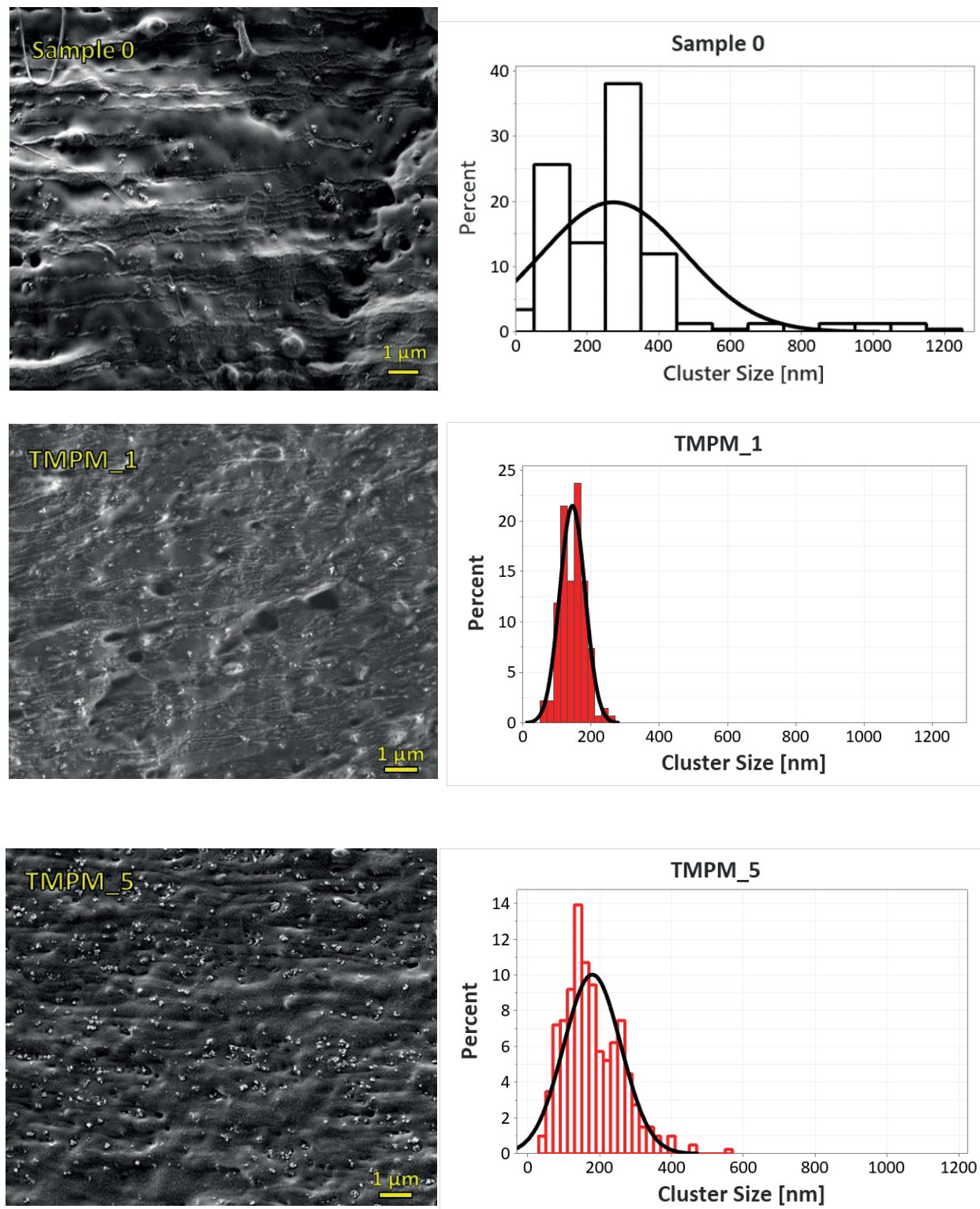

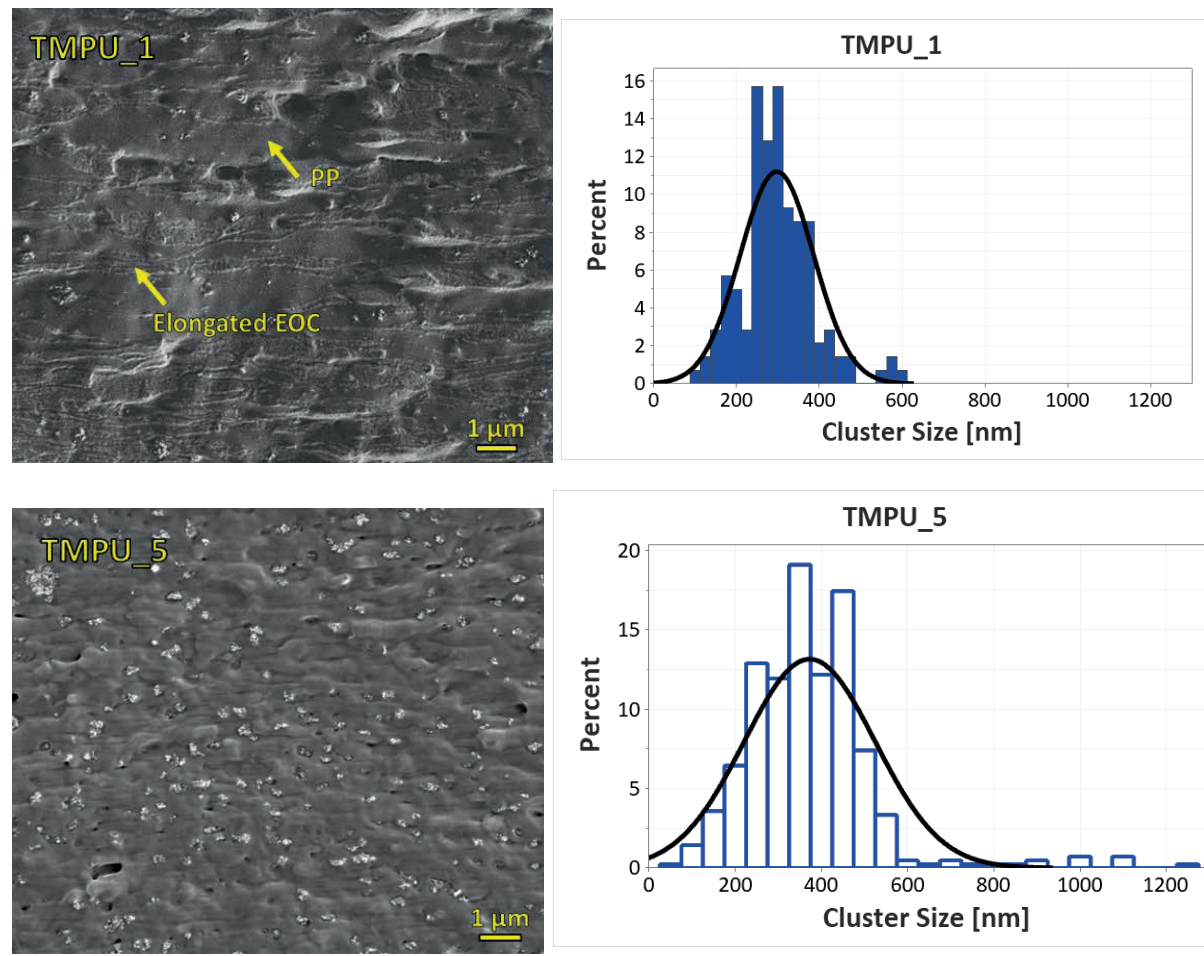

Figure 4.4. SEM images of the NCs' cross section to visualize the silica dispersion and blend morphology, samples TMPM_ 5 and TMPU_ 5 were treated by gold sputtering for a better distinction of the NPs, while Sample 0, TMPM_1 and TMPU_1 were measured without gold sputtering to visualize the polymer phases in the blend.

The melting and crystallization behavior of the studied NCs, characterized by DSC, is presented in Figure 4.5. The melting spectra exhibit two distinct peaks at $108^{\circ} \mathrm{C}$ and $147^{\circ} \mathrm{C}$, corresponding to melting temperatures of EOC and PP components of the blend, respectively. While there is no significant differences between the melting curves in all samples (Figure 4.5a), the non-isothermal crystallization of the NCs undergoes meaningful changes upon incorporation of the NPs and their modification (Figure 4.5b). Two crystallization peaks are observed in the unfilled blend spectrum, at $110{ }^{\circ} \mathrm{C}$ and $97{ }^{\circ} \mathrm{C}$, related to the PP and EOC crystalline domains. Table 4.1 presents the DSC parameters calculated from the plots. It is evident that upon incorporation of the unmodified NPs, the onset of crystallization decreases which is despite the expected nucleating effect of NPs ${ }^{18-}$ 
19. This is likely due to the absorption of the polar antioxidant particles onto the silica surface, resulting in fewer nucleating sites in the matrix ${ }^{20-21}$. For the same reason, the degree of crystallinity is not affected by the addition of the untreated silica. Nevertheless, the modification of NPs results in a significant increase of the onset of crystallization by 4 to $7^{\circ} \mathrm{C}$, as well as the degree of crystallization, which indicates the higher nucleating effect of the silica upon modification. The degree of supercooling $(\Delta T)$ can be defined as the difference between the melting and crystallization peak temperatures at a given cooling rate. This is calculated for the PP domains and presented in Table 4.1. It is evident that the modification of silica results in lower $\Delta T$ which is another indication of a more efficient nucleation in the modified NCs ${ }^{22}$. Moreover, increasing the filler content from $1 \%$ to $5 \%$ in both TMPM and TMPU cases, results in a decrease in the degree of crystallization by $2 \%$. This can be due to the slight increase in cluster size when increasing the filler content, which can result in a lower degree of crystallization ${ }^{18}$. 
a.

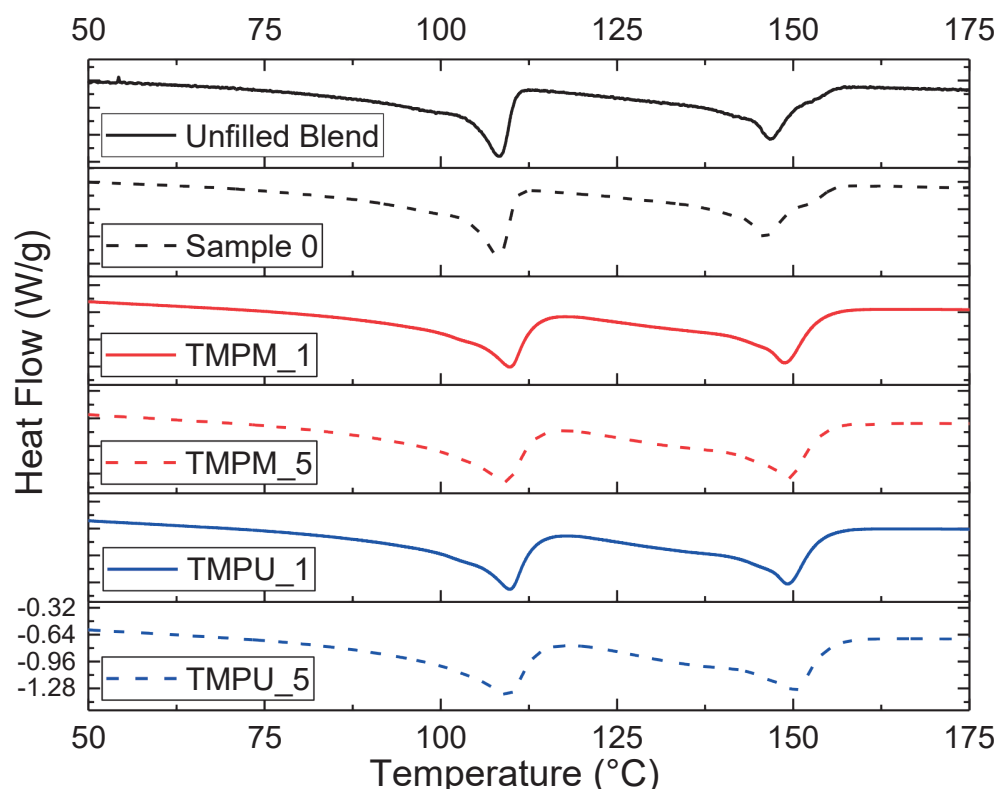

b.

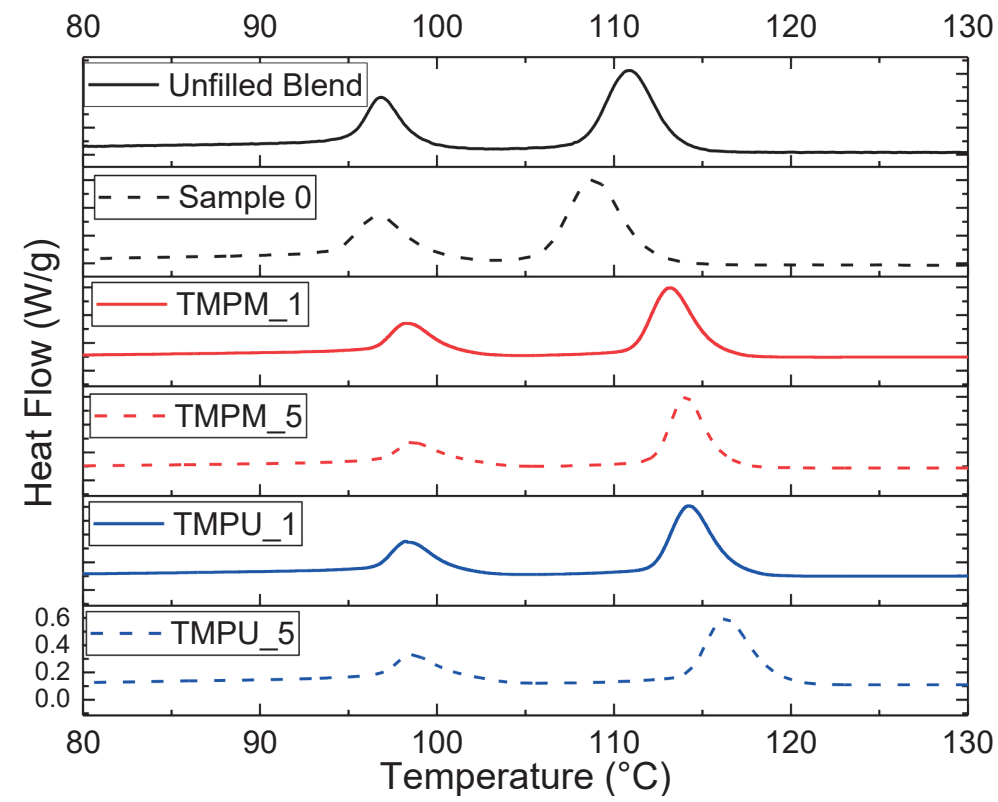

Figure 4.5. a: Melting and b: crystallization spectra of the studied NCs. 
Table 4.1. DSC parameters calculated for different NC samples

\begin{tabular}{rcccc} 
& $\begin{array}{c}\text { Onset of } \\
\text { Crystallization } \\
\left({ }^{\circ} \mathrm{C}\right)\end{array}$ & $\begin{array}{c}\text { Enthalpy of } \\
\text { Melting } \\
(\mathrm{J} / \mathrm{g})\end{array}$ & $\begin{array}{c}\text { Percentage of } \\
\text { Crystallinity } \\
(\%)\end{array}$ & $\begin{array}{c}\text { Degree of } \\
\text { Supercooling } \\
\Delta \mathbf{T}\left({ }^{\circ} \mathrm{C}\right)\end{array}$ \\
\hline Unfilled Blend & 114 & 77.3 & 31 & 36.2 \\
Sample 0 & 112 & 74.9 & 30 & 37.4 \\
TMPM_1 & 116 & 109.7 & 44 & 35.9 \\
TMPM_5 & 117 & 104.1 & 42 & 34.9 \\
TMPU_1 & 117 & 110.9 & 44 & 34.7 \\
TMPU_5 & 119 & 104.3 & 42 & 33.8
\end{tabular}

Broadband dielectric spectroscopy (BDS) is a powerful method to study the permittivity and frequency-dependent relaxations in a nanodielectric, and can provide information about interfacial polarizations and space charge formation in the material. Figures $4.6 \mathrm{a}$ and $4.6 \mathrm{~b}$ present the variations in the real part of permittivity for the TMPM and TMPU-modified NCs compared to the reference samples. In the frequency range above $1 \mathrm{~Hz}$, it is clear that with $1 \%$ of filler concentration the TMPM modification exhibits lower permittivity values compared to the TMPU-modified NCs and the reference samples. This can be due to the higher grafting density of the TMPU silane on the NPs which results in a higher number of polarizable species in the system, and hence, higher permittivity. Increasing the filler content to $5 \%$, on the one hand, decreases the permittivity of the NCs, compared to their $1 \%$ counterparts. On the other hand, TMPU_5 appears at lower levels of real permittivity compared to TMPM_5. The ureido groups in TMPU can interact strongly with each other and with other polar species in the system (e.g. antioxidant particles) via hydrogen bonds, and when incorporated at higher concentrations, can hinder the polarization mechanisms in the NC.

At the lower range of frequencies (below $1 \mathrm{~Hz}$ ), a significant rise is observed in both parts of permittivity for the unfilled blend and Sample 0 . The low frequency variations are related to either polarization of large dipolar species, or the occurrence of Maxwell-Wagner 
(space charge) relaxations ${ }^{23}$. It is clear that the introduction of either modifying agent to the NPs results in a decrease in this low frequency rise, suggesting lower susceptibility to space charge formation at their interface with the polymer matrix. This effect is pronounced when increasing the filler concentration to $5 \%$, when only a marginal increase is observed in the low frequency range for both modified samples. It can be noted that the TMPU modification results in lower interfacial polarizations compared to the TMPM. This is likely due to two reasons: firstly, the higher grafting density of TMPU results in a higher number of functional groups at the filler-polymer interface, which induces a pronounced effect in suppressing the space charge relaxations. Secondly, it has been shown that the nitrogen containing functional groups are prone to suppress space charge formation in NCs ${ }^{24-25}$. Therefore, it can be assumed that the ureido groups in the TMPU structure can induce the same effect. 
a.

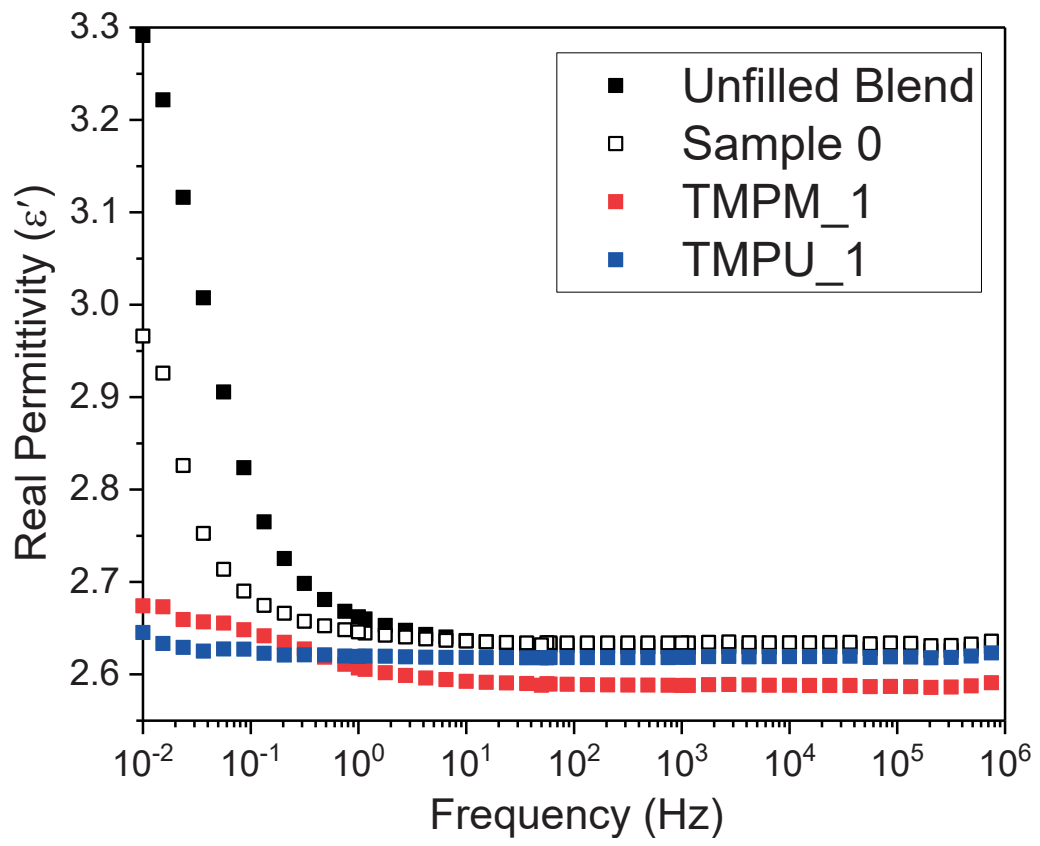

b.

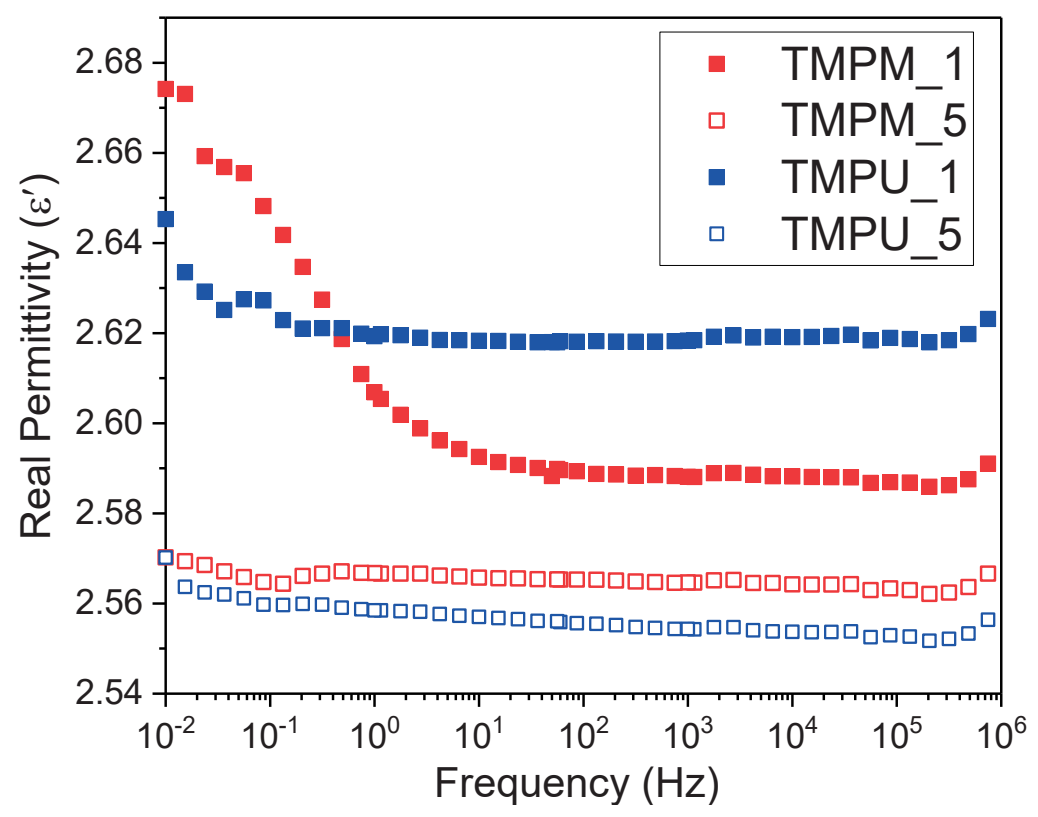


c.

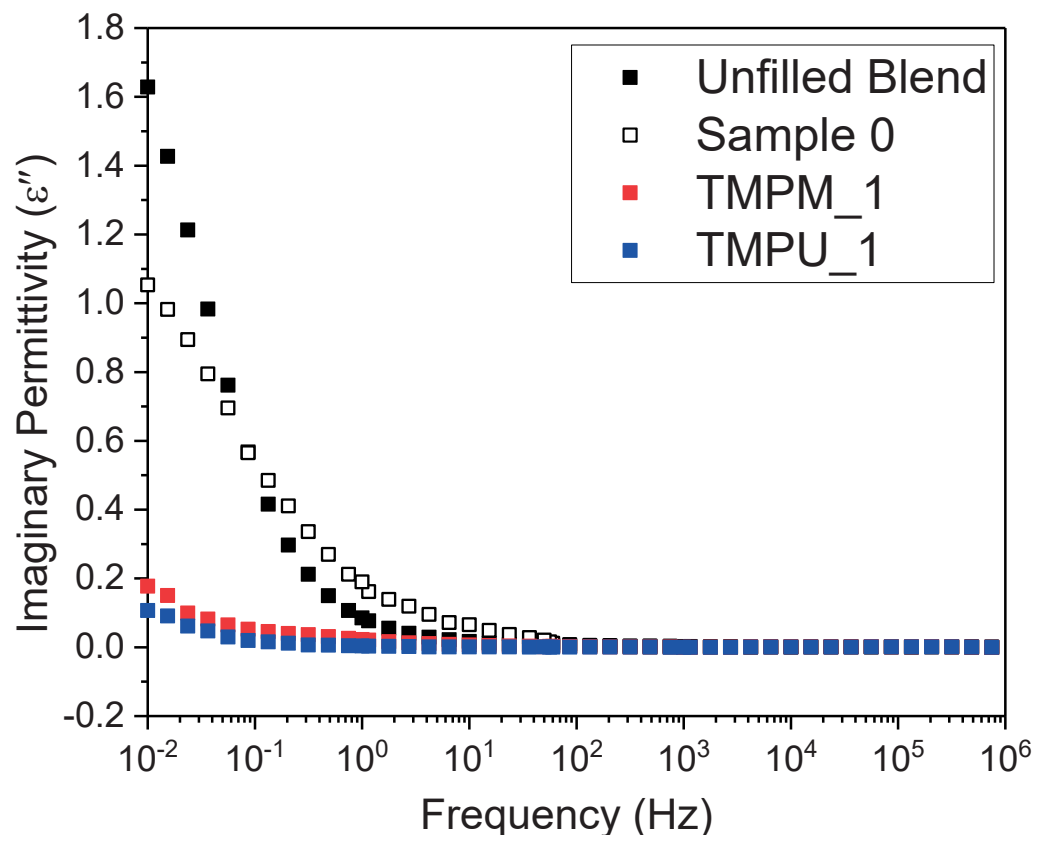

d.

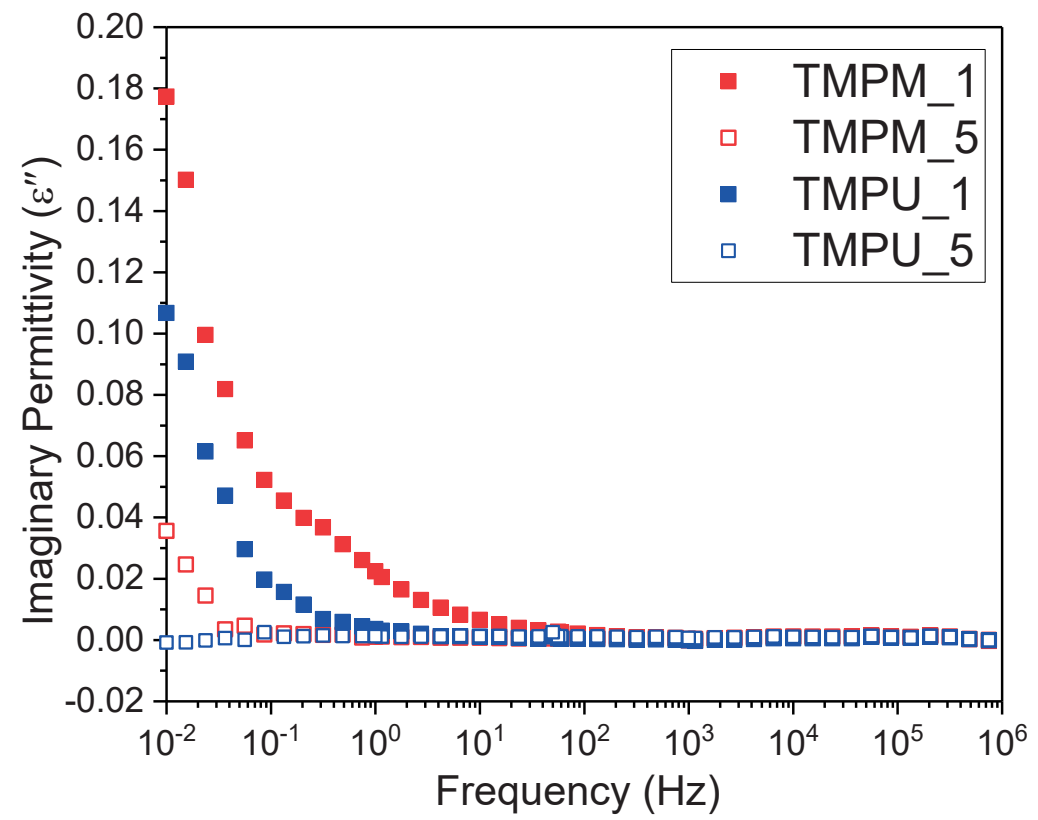

Figure 4.6. $a$ and b: real and c and d: imaginary parts of permittivity for the modified samples compared to the unfilled and untreated references 
The TSDC spectra of all studied NCs are presented in Figure 4.7a. In principle, TSDC is able to detect low-frequency motions in the material by thermal stimulation of a charged sample and monitoring the discharge current ${ }^{26}$. The discharge current at elevated temperatures, generally above the glass transition temperature of the polymer, can be attributed to the relaxation of space charge accumulated at the interfaces within the NC. Accordingly, the temperature of the peak and the corresponding current can be correlated to the depth and the density of charge traps in the NC using the theory of modified TSDC (MTSDC) reported by Tian et. al ${ }^{27}$. This is presented in Figure $4.7 \mathrm{~b}$. It can be seen that the unfilled blend exhibits a space charge relaxation peak at $75^{\circ} \mathrm{C}$ corresponding to a trap depth distribution around $0.98 \mathrm{eV}$. These are the localized states resulting from the structural defects in the polymer matrix as well as the physical defects related to antioxidant particles. The addition of untreated silica (Sample 0) slightly shifts the traps to higher energy levels indicating new localized states existing in the NPs lattice structure and at the filler-polymer interfaces. With $1 \%$ of filler concentration, TMPM-modified silica introduces shallower traps compared to the untreated NPs. Evidently, the methacrylate groups on the NP surface induce shallow trapping states, whereas the ureido functionality in TMPU-modified silica creates deep traps with energies around $1.05 \mathrm{eV}$. The deepening of the trapping states in case of TMPU is very similar to the effect of amino functionalized NPs in observed in the previous chapter ${ }^{24-25}$. This can lead to a similar conclusion that these nitrogen containing functional groups can induce deep traps at the filler-polymer interfaces. Moreover, a lower density of occupied traps is observed for the TMPU-modified silica compared to TMPM. This indicates that with the presence of ureido groups at the interfaces, the amount of charge injected to the NC is reduced which is better visualized in Figure $4.7 \mathrm{~d}$. This is also in-line with our previous observations and indicates the hampering of space charge formation in the presence of nitrogen containing functional groups at the filler-polymer interfaces. This suggests that the space charge phenomena in nanodielectrics can significantly be affected by the change in the electronic structure of the filler surface. This would enable tailoring the filler-polymer interfaces in the nanodielectric without inducing chemical bonds between the filler and the polymer. Increasing the filler concentration to $5 \%$ results in a reduction of trap densities compared to the $1 \% \mathrm{NCs}$. This reduction is most significant in 
case of TMPU_5, where the trap density is three orders of magnitude smaller compared to TMPU_1 (Figure 4.7b). As was shown in the SEM analysis, the TMPM modification resulted in a better dispersion of the NPs compared to TMPU. Nevertheless, the TMPU-modified NCS exhibit less susceptibility for space charge formation under electric fields. This suggests that the charge transport and space charge phenomena in these systems are more significantly affected by the chemical composition of the NPs' surface, rather than their dispersion quality. Figure 4.7c presents the current densities measured for all the studied samples during the poling step of the TSDC test. In general, increasing the filler concentration results in an increased number of conduction pathways due to the interference of the interfacial areas ${ }^{28}$ which would potentially result in an increased current density. However, in our case, due to the surface functionalization and the introduction of relatively deep localized states at the filler-polymer interfaces, the current density is reduced upon increasing the filler content. This suggests that at higher filler concentrations, even though more conduction pathways are accessible to the charge carriers, the trapping processes prevail. This results in a lower mobility of the charge carriers and a reduced current density in the NCs, as can be seen in Figure 4.7c. This effect is more clearly observed in comparing TMPM_5 and TMPU_5 NCs. The reduction in current density compared to their $1 \%$ counterparts is more pronounced in case of TMPU modification. This is due to the presence of deeper localized states which results in a lower mobility of charge carriers, and hence, lower current density for TMPU compared to TMPM. 
a.

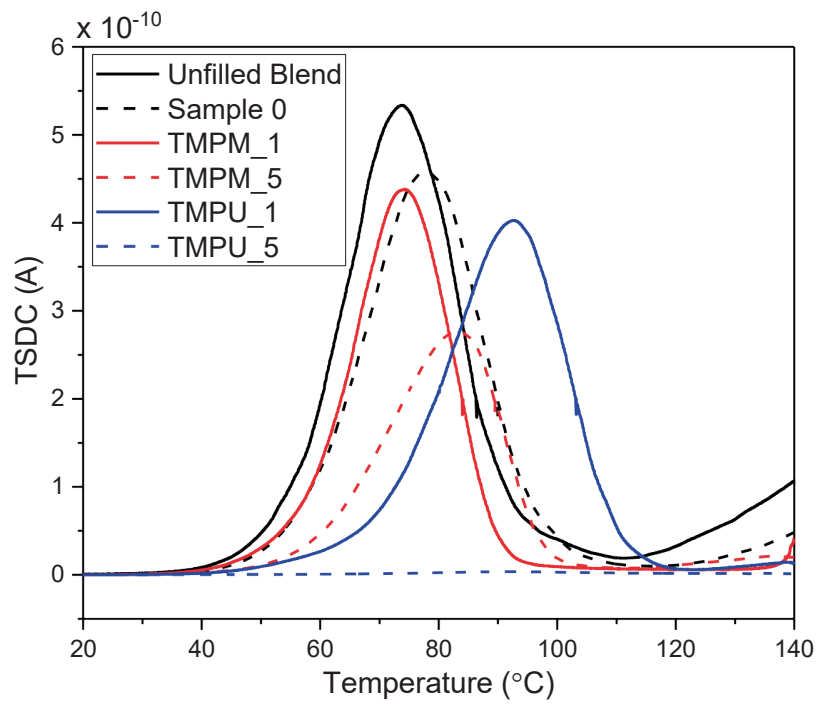

b.

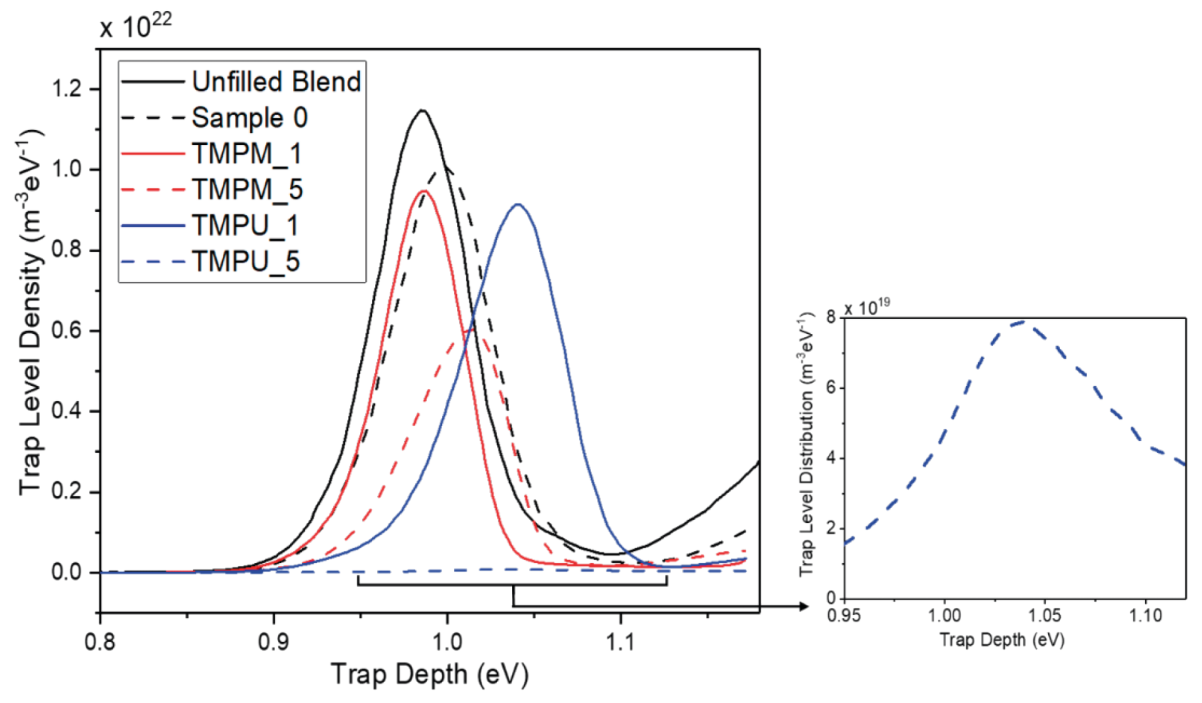


C.

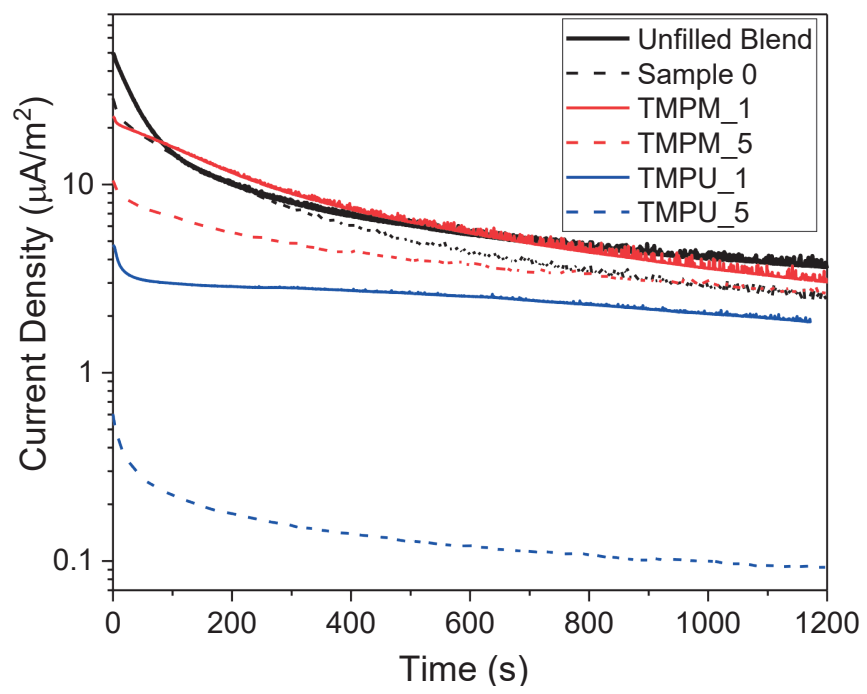

d.

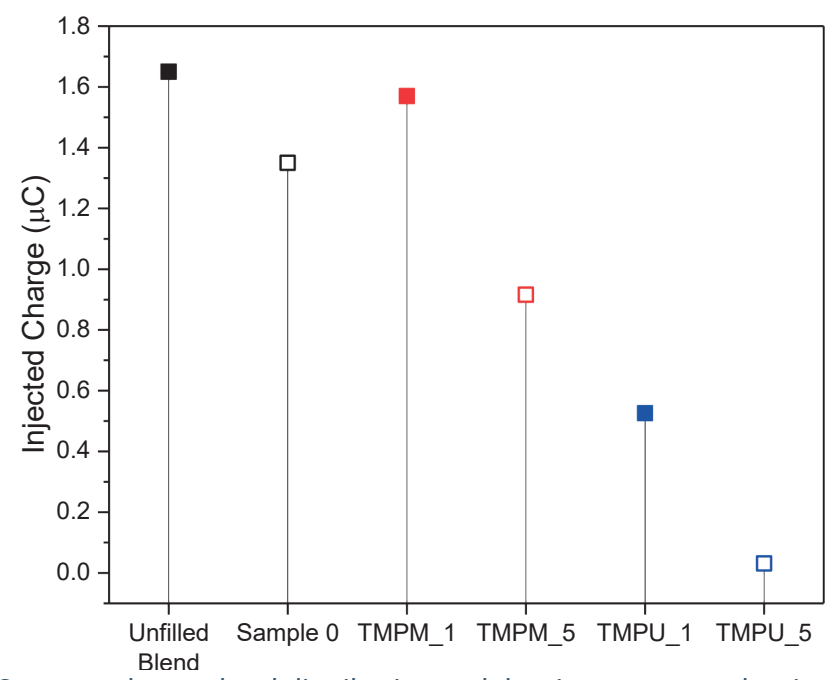

Figure 4.7. a: TSDC spectra, b: trap level distribution and density, c: current density during poling and $\mathrm{d}$ : amount of injected charge during poling for all NCs and the unfilled reference.

\section{Conclusions}

We demonstrated the successful modification of silica NPs using 3(trimethoxysilyl)propyl methacrylate (TMPM) and 1-[3-(trimethoxysilyl)propyl]urea (TMPU) as modifying agents. Subsequently, nanodielectrics based on polypropylene (PP)/ethyleneoctene-copolymer (EOC) blends were analyzed. A two-phase morphology, typical for this polymer blend, was observed. Filler dispersion was shown to be improved when TMPM- 
modified NPs were incorporated into the polymer matrix. The ureido and methacrylate functional groups introduced localized states with different energy levels. Nitrogen containing ureido groups in TMPU tend to induce deeper traps to the filler-polymer interfaces with a lower density, compared to the methacrylate silane modification. Furthermore, the TMPU modification hindered the formation of space charge at the interfaces more effectively than TMPM, even though the latter resulted in a relatively better dispersion of NPs. Increasing the filler concentration resulted in further reduction of trap densities, and hence a reduced space charge formation for both types of modification.

\section{References}

[1] Vansant, E. F.; Van Der Voort, P.; Vrancken, K. C., Characterization and chemical modification of the silica surface Elsevier: 1995; Vol. 93.

[2] Kasperovich, V.; Wong, K.; Tikhonov, G.; Kresin, V. V., Electron Capture by the Image Charge of a Metal Nanoparticle. Physical Review Letters, 85 (13), 2000, pp 2729-2732. [3] Ma, D.; Hugener, T. A.; Siegel, R. W.; Christerson, A.; Mårtensson, E.; Önneby, C.; Schadler, L. S., Influence of nanoparticle surface modification on the electrical behaviour of polyethylene nanocomposites. Nanotechnology, 16 (6), 2005, pp 724-731.

[4] Singha, S.; Thomas, M. J., Influence of filler loading on dielectric properties of epoxy-ZnO nanocomposites. IEEE Transactions on Dielectrics and Electrical Insulation, 16 (2), 2009, pp 531-542.

[5] Kozako, M.; Yamano, S.; Kido, R.; Ohki, Y.; Kohtoh, M.; Okabe, S.; Tanaka, T. Preparation and preliminary characteristic evaluation of epoxy/alumina nanocomposites, Proceedings of 2005 International Symposium on Electrical Insulating Materials, 2005.(ISEIM 2005). IEEE: 2005; pp 231-234.

[6] Calebrese, C.; Hui, L.; Schadler, L. S.; Nelson, J. K., A review on the importance of nanocomposite processing to enhance electrical insulation. IEEE Transactions on Dielectrics and Electrical Insulation, 18 (4), 2011, pp 938-945.

[7] Tan, D.; Tuncer, E.; Cao, Y.; Irwin, P. Nanofiller dispersion in polymer dielectrics, 2012 Annual Report Conference on Electrical Insulation and Dielectric Phenomena, 2012; pp 916-918.

[8] Tanaka, T.; Montanari, G. C.; Mulhaupt, R., Polymer nanocomposites as dielectrics and electrical insulation-perspectives for processing technologies, material characterization and future applications. IEEE Transactions on Dielectrics and Electrical Insulation, 11 (5), 2004, pp 763-784.

[9] Malec, D.; Truong, V.; Essolbi, R.; Hoang, T., Carrier mobility in LDPE at high temperature and pressure. IEEE Transactions on Dielectrics and Electrical Insulation, 5 (2), 1998, pp 301-303.

[10] Ma, D.; Akpalu, Y. A.; Li, Y.; Siegel, R. W.; Schadler, L. S., Effect of titania nanoparticles on the morphology of low density polyethylene. Journal of Polymer Science Part B: Polymer Physics, 43 (5), 2005, pp 488-497. 
[11] Roy, M.; Nelson, J. K.; MacCrone, R. K.; Schadler, L. S., Candidate mechanisms controlling the electrical characteristics of silica/XLPE nanodielectrics. Journal of Materials Science, 42 (11), 2007, pp 3789-3799.

[12] Ignacio, A.-C.; Verena, K.; Curtis, R.; Kevin W, E.; Johannes, S.; Cardona, A.; Seung, H. S., Trainable Weka Segmentation: a machine learning tool for microscopy pixel classification. Bioinformatics, 33 (15), 2017, pp 2424-2426.

[13] Mahtabani, A.; La Zara, D.; Anyszka, R.; He, X.; Paajanen, M.; van Ommen, J. R.; Dierkes, W.; Blume, A., Gas Phase Modification of Silica Nanoparticles in a Fluidized Bed: Tailored Deposition of Aminopropylsiloxane. Langmuir, 37 (15), 2021, pp 4481-4492.

[14] Brochier Salon, M.-C.; Bayle, P.-A.; Abdelmouleh, M.; Boufi, S.; Belgacem, M. N., Kinetics of hydrolysis and self condensation reactions of silanes by NMR spectroscopy. Colloids and Surfaces A: Physicochemical and Engineering Aspects, 312 (2), 2008, pp 83-91.

[15] Grabowski, S. J., Ab Initio Calculations on Conventional and Unconventional Hydrogen BondsStudy of the Hydrogen Bond Strength. The Journal of Physical Chemistry A, 105 (47), 2001, pp 10739-10746.

[16] Halvorson, R. H.; Erickson, R. L.; Davidson, C. L., The effect of filler and silane content on conversion of resin-based composite. Dental Materials, 19 (4), 2003, pp 327 333.

[17] Xie, C.; Zeng, X.; Fang, W.; Lai, X.; Li, H., Effect of alkyl-disubstituted ureido silanes with different alkyl chain structures on tracking resistance property of addition-cure liquid silicone rubber. Polymer Degradation and Stability, 142, 2017, pp 263-272.

[18] Saujanya, C.; Radhakrishnan, S., Structure development and crystallization behaviour of PP/nanoparticulate composite. Polymer, 42 (16), 2001, pp 6723-6731.

[19] Vladimirov, V.; Betchev, C.; Vassiliou, A.; Papageorgiou, G.; Bikiaris, D., Dynamic mechanical and morphological studies of isotactic polypropylene/fumed silica nanocomposites with enhanced gas barrier properties. Composites Science and Technology, 66 (15), 2006, pp 2935-2944.

[20] Mahtabani, A.; He, X.; Rytöluoto, I.; Lahti, K.; Paajanen, M.; Saarimäki, E.; Anyszka, R.; Dierkes, W.; Blume, A. Effect of silica modification on charge trapping behavior of PP blend/silica nanocomposites, 2019 2nd International Conference on Electrical Materials and Power Equipment (ICEMPE), IEEE: 2019; pp 241-245.

[21] Binsbergen, F. L., Heterogeneous nucleation in the crystallization of polyolefins: Part 1. Chemical and physical nature of nucleating agents. Polymer, 11 (5), 1970, pp 253267.

[22] Jain, S.; Goossens, H.; van Duin, M.; Lemstra, P., Effect of in situ prepared silica nano-particles on non-isothermal crystallization of polypropylene. Polymer, 46 (20), 2005, pp 8805-8818.

[23] Nelson, J. K.; Fothergill, J. C., Internal charge behaviour of nanocomposites. Nanotechnology, 15 (5), 2004, p 586.

[24] Mahtabani, A.; Rytöluoto, I.; Anyszka, R.; He, X.; Saarimäki, E.; Lahti, K.; Paajanen, M.; Dierkes, W.; Blume, A., On the Silica Surface Modification and Its Effect on Charge Trapping and Transport in PP-Based Dielectric Nanocomposites. ACS Applied Polymer Materials, 2 (8), 2020, pp 3148-3160.

[25] He, X.; Rytöluoto, I.; Anyszka, R.; Mahtabani, A.; Saarimäki, E.; Lahti, K.; Paajanen, M.; Dierkes, W.; Blume, A., Silica Surface-Modification for Tailoring the Charge Trapping 
Properties of PP/POE Based Dielectric Nanocomposites for HVDC Cable Application. IEEE Access, 8, 2020, pp 87719-87734.

[26] Turnhout, J. v., Thermally Stimulated Discharge of Polymer Electrets. Polymer Journal, 2 (2), 1971, pp 173-191.

[27] Tian, F.; Bu, W.; Shi, L.; Yang, C.; Wang, Y.; Lei, Q., Theory of modified thermally stimulated current and direct determination of trap level distribution. Journal of Electrostatics, 69 (1), 2011, pp 7-10.

[28] Yang, X.; Hu, J.; Chen, S.; He, J., Understanding the Percolation Characteristics of Nonlinear Composite Dielectrics. Scientific Reports, 6 (1), 2016, p 30597. 



\section{CHAPTER 5}

Gas Phase Modification of Silica Nanoparticles in a Fluidized Bed 


\begin{abstract}
$^{1}$
Functionalized nanoparticles have various applications, for which grafting of a chemical moiety onto the surface to induce/improve certain properties is needed. When incorporated in polymeric matrices, for instance, the modified nanoparticles can alter the interfacial characteristics leading to improvements over the macroscopic properties of the nanocomposites. The extent of these improvements is highly dependent on the thickness, morphology and conformity of the grafted layer. However, the common liquid-phase modification methods provide limited control over these factors. A novel gas-phase modification process was utilized, with 3-aminopropyltriethoxysilane (APTES) as precursor, to chemically deposit amino-terminated organic layers on fumed silica nanoparticles in a fluidized bed. A self-limiting surface saturation was achieved when the reaction was done at $200{ }^{\circ} \mathrm{C}$. With this self-limiting feature, we were able to graft multiple layers of aminopropylsiloxane (APS) onto the silica nanoparticles using water as the co-reactant. The feasibility of this process was analyzed using Thermogravimetric Analysis (TGA), Diffuse Reflectance IR Fourier Transform Spectroscopy (DRIFTS), X-Ray Photoelectron Spectroscopy (XPS) and Elemental Analysis (EA). By altering the number of APTES/water cycles, it was possible to control the thickness and conformity of the deposited aminopropylsiloxane layer. This novel approach allows to engineer the surface of nanoparticles, by introducing versatile functionalized layers in a controlled manner.
\end{abstract}

1 The results of this chapter are published in Langmuir, 37, 15, pp. 4481-4492, 2021 (https://doi.org/10.1021/acs.langmuir.0c03647) 


\section{Introduction}

For various industrial and scientific applications, there is a need to functionalize the surfaces of nanoparticles in order to achieve a variety of properties. Among different types of nanoparticles, silica is widely used in many applications as filler ${ }^{1-3}$, catalyst carrier ${ }^{4}$, and biological and medical materials ${ }^{5}$. In order to improve the application performance, their surfaces usually need to be modified by functional chemical groups 6-7 . 3aminopropyltriethoxysilane (APTES) is a well-studied and commonly used modifying agent because of its versatility and wide application range. Amino-terminated silica particles can be used as filler in rubber and plastic matrices to improve mechanical properties by enhancing the filler-matrix interactions ${ }^{8}$. Amino-terminated silica particles also have a large adsorption capacity and good selectivity for metal ions, such as $\mathrm{Cu}^{2+}, \mathrm{Pb}^{2+}, \mathrm{Hg}^{2+}$, thus they can be used as chromatography stationary phase or adsorbent ${ }^{9-11}$. Since amino groups can react with proteins and DNA, the functionalized particles have important applications in the separation of biomaterials, enzyme immobilization and targeted medicine ${ }^{12-13}$.

In Chapter 3, it was shown that treating silica nanoparticles with APTES results in a significant reduction of space charge injection in nanodielectrics under DC fields. It was also discussed that the amine functional group can induce deep localized states that can trap the mobile charges when the DC field is applied ${ }^{14}$. Also, substituting the silanol groups with amine functional groups on the silica can reduce the surface polarity, minimize the dispersion challenges and reduce moisture uptake ${ }^{15}$. Liu et al. ${ }^{16-17}$ have shown that the mass of adsorbed moisture and medium sized species, such as byproducts from filler modification and polymer crosslinking, is proportional to the hydroxide concentration on the nanoparticle surface. It has also been shown that the long-term deterioration of insulation properties of polymeric nanocomposites can be due to uncontrolled moisture uptake ${ }^{18}$. Therefore, depositing conformal aminopropylsiloxane (APS) layers from APTES on the silica nanoparticles in a controlled manner appears to be a promising way to achieve and maintain good insulation properties.

The preparative method of the functionalized nanoparticles has a remarkable effect on the coating morphology, i.e. layer thickness, surface density, orientation of the surface 
molecules, and the type of interactions between the surface groups and the precursor molecules ${ }^{19}$. Functionalization of nanoparticles is usually done in an aqueous or nonaqueous liquid phase, with organic solvents, e.g. toluene, hexane or ethanol. This method, while proven to be effective ${ }^{20}$, brings about serious issues such as solvent recovery, long operation times, high costs, severe pollution, and low efficiency ${ }^{21}$. Moreover, the presence of water has a great influence on the mechanism of layer formation and further on the structure of the deposited layer ${ }^{22}$. In aqueous conditions, multilayers are formed by oligomerization of bi- and trifunctional alkoxysilanes due to hydrogen-bonding and hydrolysis of precursor molecules before deposition onto the surface via covalent bonds ${ }^{19}$. This usually occurs inevitably and in spite of careful dehydration treatments ${ }^{23}$, hence making it difficult to control the thickness and morphology of the deposited layers. Elimination of the physisorbed water molecules and deposition of self-saturating molecular layers of alkoxysilanes can be most easily performed in the gas phase. Different silane layers have been deposited onto silica powders in the gas phase by means of carrier particles containing a known quantity of modifying agent ${ }^{21,24}$. However, this approach does not provide control over the thickness and morphology of the deposited layer. Aminosilane layers have been deposited onto silicon dioxide substrates in gas phase via atomic layer deposition (ALD) ${ }^{25}$. Hydrolysis of aminosilylated silica, that is hydrolyzing the remaining unreacted alkoxy groups with water, followed by a silanization step resulted in the controlled formation of a high-density amino-terminated siloxane network. When consecutive aminopropyltrialkoxysilane and water cycles have been performed, an increased content of amino groups on porous silica is observed. To our knowledge, this approach has not yet been applied on nanoparticles in a fluidized bed.

In this chapter, fumed silica nanoparticles were treated in the gas phase in a fluidized bed with 3-aminopropyltriethoxysilane (APTES). APTES reacts with the isolated and geminal silanol groups on the silica in a self-catalyzing manner due to hydrogen bond interactions ${ }^{26}$. Therefore, the first layer can be chemically deposited onto the nanoparticles' surface with no need for hydrolysis. Figure 5.1a depicts a schematic presentation of this reaction. Different reaction temperatures were applied in order to verify self-limiting surface saturation. Subsequently, multiple layers were deposited onto the nanoparticles via 
sequential APTES/water cycles (Figure 5.1b). This process is similar to that of atomic layer deposition (ALD), where layers are formed on a reactive surface by sequential pulses of two precursors in the gas phase. In this way, deposition of APTES can be controlled, layer by layer, by increasing the number of APTES/water cycles. The feasibility of this approach was analyzed using Thermogravimetric Analysis (TGA), Diffuse Reflectance Infrared Fourier Transform Spectroscopy (DRIFTS), X-Ray Photoelectron Spectroscopy (XPS) and Elemental Analysis (EA).
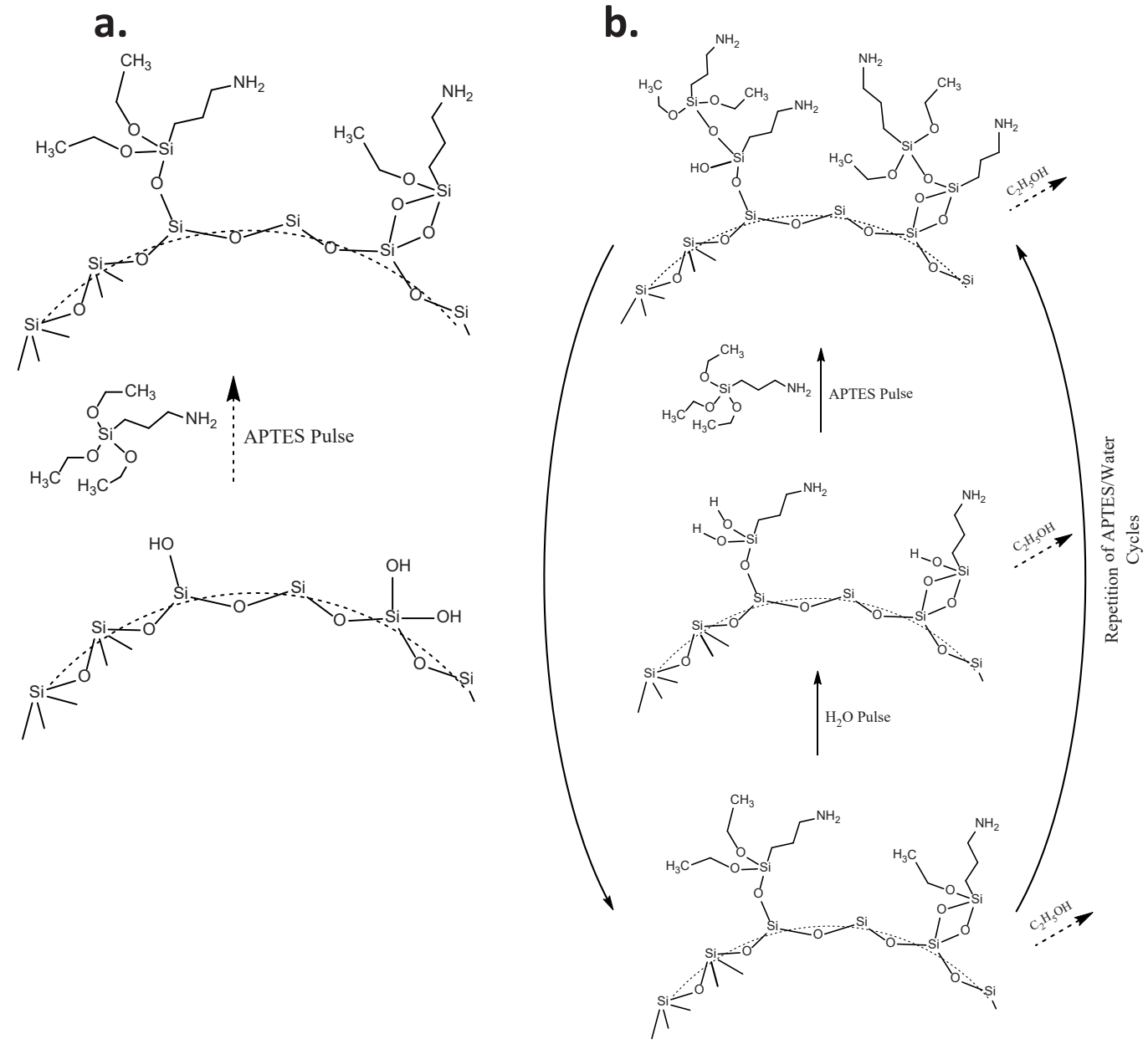

Figure 1. a: Deposition mechanism of aminopropylsiloxane onto the silica nanoparticles, b:

Repetition of the APTES/water cycles to achieve conformal coating of the surface. 


\section{Experimental Work}

\subsection{Materials}

A fumed silica grade, AEROSIL 200 (primary particle size of 7-50 nm) with high purity and low moisture content, was received from Evonik Industries (Germany). 3aminopropyltriethoxysilane (APTES) with $99 \%$ purity was purchased from Sigma Aldrich (Germany) and used without any further purification. In order to dry the silica and rule out the effect of air humidity, nanoparticles were kept in an oven at $120^{\circ} \mathrm{C}$ for $24 \mathrm{~h}$ prior to the modification process.

\subsection{Gas Phase Deposition of the Silane}

The experiments were carried out in a fluidized bed reactor operated at atmospheric pressure: the particles are suspended ("fluidized") in an upward gas flow. The reactor consists of a glass column $(2.6 \mathrm{~cm}$ in internal diameter and $50 \mathrm{~cm}$ in height), placed on top of a double-motor vibration table (Paja PTL 40/40-24, Netherlands) to assist the fluidization process. Two stainless-steel distributor plates with pore size of $37 \mu \mathrm{m}$ were placed at the bottom and top of the column to obtain a homogeneous distribution of the gas inside the column and to prevent particles from leaving the reactor. APTES was used as the modifying agent and was heated up to $110^{\circ} \mathrm{C}$ in a stainless-steel bubbler. In some further experiments, in order to investigate the controllability of the silane layer deposition on the fluidized silica nanoparticles, a second bubbler was added to the setup containing water at room temperature. The bubbler temperature was chosen based on the vapor pressure of the contained substance. The silica nanoparticles were fluidized and pre-treated in the glass column with nitrogen $\left(\mathrm{N}_{2}\right)$ flow for $1 \mathrm{~h}$ at the desired reaction temperature in order to break larger agglomerates of nanoparticles and remove physisorbed water. After the pretreatment, the fluidized powder bed consisted of two zones: a bottom zone with large agglomerates (up to $5 \mathrm{~mm}$ in diameter) and a top zone of fine powder, which fluidized smoothly. Subsequently, the fluidized silica was exposed to the precursors carried by nitrogen gas passing through bubblers at different pulse times. After the treatment, the fluidized powder was purged by nitrogen gas to remove by-products and physisorbed 
precursor, and the fine powder and agglomerated particles were collected and characterized separately. Since the fine powders are more effectively mixed in the bed, they better represent the properties of the modified nanoparticles with this method, and therefore, are subjected to more detailed characterizations. The details of each experiment are depicted in Table 5.1.

Table 5.1. Details of experiments performed in the fluidized bed

\begin{tabular}{|c|c|c|c|c|}
\hline $\begin{array}{c}\mathrm{T} \text { bubbler } \\
\left({ }^{\circ} \mathrm{C}\right)\end{array}$ & $\begin{array}{l}\text { tpre-treatment } \\
\quad(\min )\end{array}$ & $\begin{array}{c}T_{\text {reaction }} \\
\left({ }^{\circ} \mathrm{C}\right)\end{array}$ & $\begin{array}{c}t_{\text {reaction }} \\
\text { (min) }\end{array}$ & $\begin{array}{l}t_{\text {purging }} \\
\text { (min) }\end{array}$ \\
\hline \multirow{13}{*}{110} & \multirow{13}{*}{60} & \multirow{4}{*}{150} & 1 & \multirow{13}{*}{60} \\
\hline & & & 3 & \\
\hline & & & 5 & \\
\hline & & & 10 & \\
\hline & & & 1 & \\
\hline & & & 3 & \\
\hline & & 200 & & \\
\hline & & & 5 & \\
\hline & & & 10 & \\
\hline & & \multirow{4}{*}{250} & 1 & \\
\hline & & & 3 & \\
\hline & & & 5 & \\
\hline & & & 10 & \\
\hline
\end{tabular}

It is assumed that upon reaching a surface saturation, there are neither silanol groups on the silica nor hydrolyzed ethoxy groups of already attached silane molecules accessible for the silane to react with. A water pulse through the bed can hydrolyze the remaining ethoxy groups, and result in the growth of the deposited layer upon the next silane pulse. Therefore, it is expected that consecutive APTES/water pulses through the fluidized silica nanoparticles would result in the growth of the deposited layer upon each cycle. Each cycle 
starts with an APTES pulse for 5 minutes to deposit a sub-mono layer of the silane onto the fluidized silica nanoparticles. This step is followed by 10 minutes of nitrogen purging in order to remove the by-products and the physisorbed APTES. Subsequently, water is sent through the column for 3 minutes so that the exposed ethoxy groups become hydrolyzed and ready to react with the silane pulsed in the next cycle. Finally, the fluidized silica is purged again with nitrogen for 10 minutes.

\subsection{Characterization of the Nanoparticles}

\subsubsection{Thermogravimetric Analysis (TGA)}

In order to estimate the total number of silanol groups on the surface of the nanoparticles, Thermogravimetric Analysis (TGA 550, TA Instruments, USA) was performed on the untreated Aerosil 200. The method consists of heating up the silica powder from room temperature to $850{ }^{\circ} \mathrm{C}$ with a rate of $20^{\circ} \mathrm{C} / \mathrm{min}$ under air while measuring the mass loss of the sample. It is assumed that all the physisorbed water is released from the sample up to $150{ }^{\circ} \mathrm{C}^{27-29}$. Therefore, the total number of silanol groups for $1 \mathrm{~g}$ of silica can be calculated from the mass loss above $150{ }^{\circ} \mathrm{C}$ and using Equation 5-1:

$$
n_{\mathrm{SiOH}}\left(\frac{\mathrm{SiOH}}{n m^{2}}\right)=2 \frac{\Delta m \cdot N_{A}}{M_{\mathrm{H}_{2} \mathrm{O}} \cdot S_{A}}
$$

$n_{\mathrm{SiOH}}$ : total number of silanol groups

$\Delta m$ : mass loss in the temperature range: $150^{\circ} \mathrm{C}<\mathrm{T}<850^{\circ} \mathrm{C}$

$N_{A}$ : Avogadro's constant

$M_{\mathrm{H}_{2} \mathrm{O}}$ : molecular mass of water $(18 \mathrm{~g} / \mathrm{mol})$

$S_{A}$ : Surface area of the nanoparticles $\left(200 \mathrm{~m}^{2} / \mathrm{g}\right)$

The removal of physisorbed water from the nanoparticles is crucial to reach ALD surface saturation. However, the released water from siloxanation of the vicinal silanol groups during the process can hydrolyze the alkoxy groups of the silane, initiate oligomerization of the APTES species and disturb the self-limiting growth of the film. The 
number of siloxanating silanol groups was estimated at each reaction temperature using TGA: the untreated silica nanoparticles were rapidly $\left(50^{\circ} \mathrm{C} / \mathrm{min}\right)$ heated up to the desired temperature, and held isothermally for $1 \mathrm{~h}$. It is assumed that the heating rate during the ramp does not affect the total mass loss up to the isothermal step. The number of silanol groups siloxanating at each temperature can then be calculated from Equation 5-2:

$n_{\text {SiOH }}^{T^{*}}\left(\frac{\mathrm{SiOH}}{n m^{2}}\right)=2 \frac{\Delta m_{T} \cdot N_{A}}{M_{\mathrm{H}_{2} \mathrm{O}} \cdot S_{A}}$

(Equation 5-2)

$n_{\text {SiOH }}^{T^{*}}$ : number of silanol groups siloxanating at temperature T

$\Delta m_{T}:$ mass loss during the isothermal step

The multiplier two in Equations 1 and 2, is related to the assumption that siloxanation of two adjacent silanol groups results in release of one water molecule ${ }^{29}$. Having calculated the total number of silanol groups as well as the number of silanol groups siloxanating at each reaction temperature, one can calculate the grafting density (GD) of the APTES upon surface saturation using Equation 5-3:

$$
G D_{\text {cal }}^{\text {sat }}\left(\frac{\text { mmol APTES }}{g \text { Silica }}\right)=\frac{n_{\text {SiOH }}^{T} \cdot S_{A}}{N_{A} \times 10^{-3}}
$$

where $n_{\mathrm{SiOH}}^{T}=n_{\mathrm{SiOH}}-n_{\mathrm{SiOH}}^{T^{*}}$ is number of remaining silanol groups on the silica at temperature T, and $G D_{\text {cal }}^{\text {sat }}$ is the calculated grafting density of APTES upon saturation. Here, it is assumed that surface saturation takes place in the absence of water, and that in this case, each $\mathrm{SiOH}$ reacts only with one APTES molecule, i.e. a monodentate structure ${ }^{20}$.

TGA was also performed on the modified silica in order to quantify the level of modification. Each sample was heated up from room temperature to $850{ }^{\circ} \mathrm{C}$ with a rate of $20^{\circ} \mathrm{C} / \mathrm{min}$. The mass loss above $300^{\circ} \mathrm{C}$ was attributed to the thermal decomposition of the aminopropyl groups grafted on the silica surface ${ }^{20}$. The GD can then be calculated from the thermograms according to Equation 5-4: 
$G D_{\exp }^{\text {sat }}\left(\frac{m \text { mol APTES }}{g \text { Silica }}\right)=\frac{10^{3}}{M_{W}}\left(\frac{\Delta W}{1-\Delta W}\right)$

(Equation 5-4)

$G D_{\text {exp }}^{\text {sat }}$ : saturated grafting density of APTES from the experiment

$\Delta W\left(\frac{\text { g APS }}{\text { g sample }}\right):$ TGA mass loss between $300^{\circ} \mathrm{C}$ and $850^{\circ} \mathrm{C}$

1- $\Delta W\left(\frac{g \text { silica }}{\text { g sample }}\right)$ : Mass fraction of the pure silica in the modified sample

$M_{w}$ : Molecular mass of the aminopropyl moiety $(58 \mathrm{~g} / \mathrm{mol})$

\subsubsection{DRIFTS Measurements}

Diffuse Reflectance IR Fourier Transform Spectroscopy (DRIFTS) was utilized to further evaluate the deposition of APTES. Analyses were conducted using a Nicolet 8700 spectrometer (Thermo Fisher Scientific) equipped with a DTGS detector. The samples were prepared using $\mathrm{KBr}$ (99+\%, FTIR grade, Harrick Scientific Corporation) as background. Spectra were recorded from $4000-400 \mathrm{~cm}^{-1}$ and averaged over 128 scans, using a resolution of $4.0 \mathrm{~cm}^{-1}$. The data is plotted in the wavenumber range of $4000-1500 \mathrm{~cm}^{-1}$ as this is the range of interest in this case. All the tests were performed at room temperature.

\subsubsection{X-Ray Photoelectron Spectroscopy (XPS)}

X-ray Photoelectron Spectroscopy (XPS) was conducted as to the description in Chapter 3.

\subsubsection{Elemental Analysis}

Elemental analysis was done using a Thermo Scientific Flash 2000 organic elemental analyzer. Small amounts of the powder were put into tin containers and placed inside the equipment chambers. Subsequently, the sample was heated up till $1000^{\circ} \mathrm{C}$, and the weight percent of present elements was measured. 


\section{Results and Discussions}

\subsection{Quantification of the Silanol Groups}

Figure 5.2 shows the thermogram of the untreated silica heated from room temperature to $850{ }^{\circ} \mathrm{C}$. Distinct steps in the mass loss of silica can be observed from the derivative curve. The two steps, reaching their maximum rate first before $100{ }^{\circ} \mathrm{C}$ and then around $150{ }^{\circ} \mathrm{C}$, are mainly due to the release of the physisorbed water. This observation validates the assumption used in Equation 5-1, and enables one to calculate the total number of silanol groups on the silica. The mass loss of $1.2 \%$ above $150{ }^{\circ} \mathrm{C}$, accounts for $4 \mathrm{SiOH} / \mathrm{nm}^{2}$ according to Equation $5-1$, which is in accordance with what is measured by other methods ${ }^{29}$. According to the mass loss derivative graph in Figure 5.2, the release of silanol groups in form of water reaches its maximum rate first around $150{ }^{\circ} \mathrm{C}$ and then again around $300{ }^{\circ} \mathrm{C}$. Therefore, in order to avoid APTES oligomerization and reach surface saturation, it is crucial to perform the modification reaction in the $150-300{ }^{\circ} \mathrm{C}$ temperature range. The high mass loss rate above $150{ }^{\circ} \mathrm{C}$ is mainly due to the release of chemisorbed water. This is the water released as a result of siloxanation of surface silanol groups ${ }^{29}$. The high loss rate at $300{ }^{\circ} \mathrm{C}$ is, therefore, related to further siloxanation of reactive silanol groups, which would substantially decrease the chemical deposition of APS. Accordingly, this reaction temperature can be ruled out from the modification experiments. It is also noteworthy here that, according to ${ }^{30}$, no temperature effect on the surface density of amino groups on silica has been observed at reaction temperatures of $120-150{ }^{\circ} \mathrm{C}$, when the deposition of aminosilanes was performed in the gas phase. Therefore, temperatures lower than $150{ }^{\circ} \mathrm{C}$ are not necessary to study for this process. 


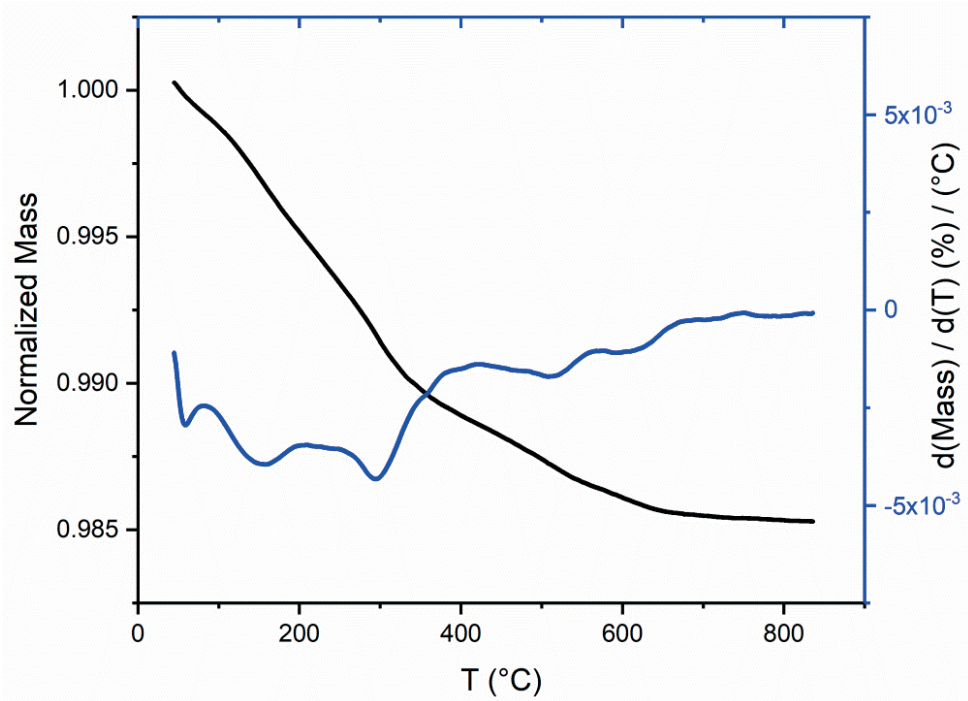

Figure 5.2. Thermogram of the untreated silica heated from room temperature to $850^{\circ} \mathrm{C}$. Since the mass decreases over time, the derivative curve has negative values

Figure 5.3 shows the thermograms of untreated silica samples when kept isothermally at each reaction temperature for $1 \mathrm{~h}$. The objective of the isothermal TGA was to determine the lowest temperature range at which the ALD-like saturation is more likely to occur. During the rapid heating, before the stabilization of the temperature, the powder samples lose water; the higher the intended isothermal temperature, the higher the silica loses its physisorbed and chemisorbed water. This can be observed in Figure 5.3: at $200{ }^{\circ} \mathrm{C}$ and $250{ }^{\circ} \mathrm{C}$, less water remains in the sample, hence, the thermograms show lower overall mass loss during the isothermal step compared to $150{ }^{\circ} \mathrm{C}$. This shows that the silica contains relatively more water at $150{ }^{\circ} \mathrm{C}$, and hence, the oligomerization of APTES species during the modification is more likely at this reaction temperature. More so, the mass loss seems not to stabilize throughout the isothermal step, implying that there is still release of water from the silica after being kept at $150{ }^{\circ} \mathrm{C}$ for $1 \mathrm{~h}$. Whereas at $200{ }^{\circ} \mathrm{C}$ and $250{ }^{\circ} \mathrm{C}$, less water is available on the silica, and the mass becomes stable during the isothermal step, indicating more likeliness for surface saturation during the treatment at these reaction temperatures. 


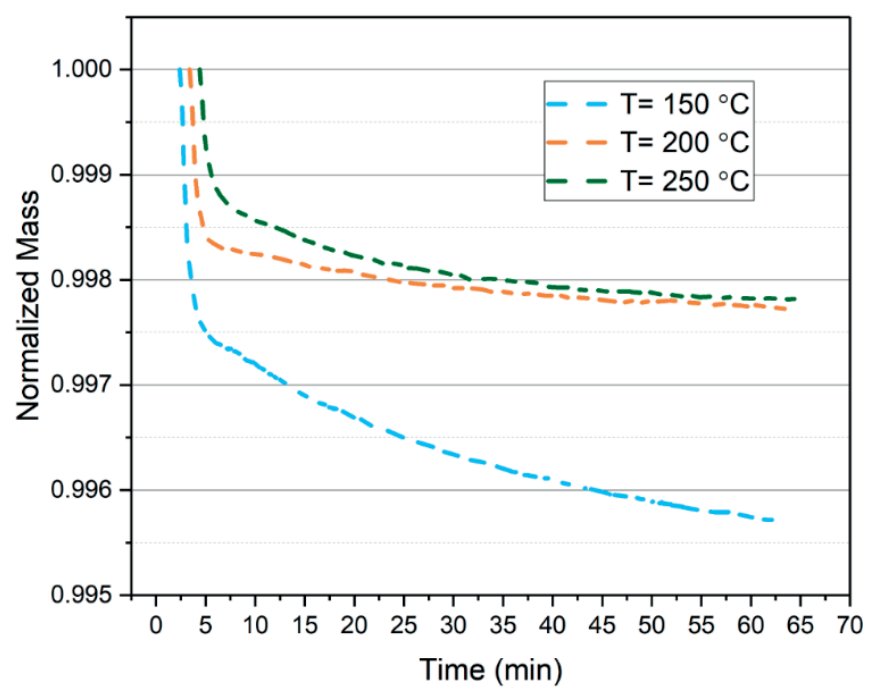

Figure 5.3. Isothermal thermograms of the untreated silica at three reaction temperatures; the mass loss at $150^{\circ} \mathrm{C}$ is significantly higher than the other two temperatures, mainly due to the continuous loss of water from the surface

\subsection{Gas-Phase Deposition of the Amino-Silane}

Having gained some perspective on the silica, the reaction of vaporized APTES with fumed silica nanoparticles was studied in a fluidized bed using the reaction conditions depicted in Table 5.1. Three reaction temperatures: $150^{\circ} \mathrm{C}, 200^{\circ} \mathrm{C}$ and $250{ }^{\circ} \mathrm{C}$, were utilized for the process in order to find the temperature at which the reaction is surface-limiting and where conformal self-saturating layers can be formed on the silica surface. This is one of the main features of an ALD process ${ }^{31-32}$, which in this case would enable for controlled deposition of aminopropylsiloxane (APS) on the silica surface. The DRIFTS spectra of all the treated samples compared to the bare silica are presented in Figure 5.4. All spectra were normalized to the $\mathrm{SiO}_{2}$ vibrational band at $1860 \mathrm{~cm}^{-1}$ as the underlying bulk ${ }^{33}$. The isolated $\mathrm{Si}-\mathrm{OH}$ band at $3750 \mathrm{~cm}^{-1}$, disappearing at pulse times longer than $1 \mathrm{~min}$, regardless of the reaction temperature, indicates the rapid consumption of the isolated silanol groups. This suggests that, after this point, it is mostly the siloxanation of geminal and vicinal silanol groups, that determines the reaction mechanisms. The intensity of the $\mathrm{C}-\mathrm{H}$ stretching bands at $2870-2970 \mathrm{~cm}^{-1}$ increases upon increasing APTES pulse time at $150{ }^{\circ} \mathrm{C}$ (Figure $5.4 \mathrm{a}$ ), which is not observed in case of $200{ }^{\circ} \mathrm{C}$ and $250{ }^{\circ} \mathrm{C}$. This is an indication of the non-saturated 
deposition at $150{ }^{\circ} \mathrm{C}$ as opposed to the obtained saturation at higher temperatures. The same increasing trend at $150{ }^{\circ} \mathrm{C}$ is observed for the $\mathrm{N}-\mathrm{H}$ bending band at $1590 \mathrm{~cm}^{-1}$.

a.

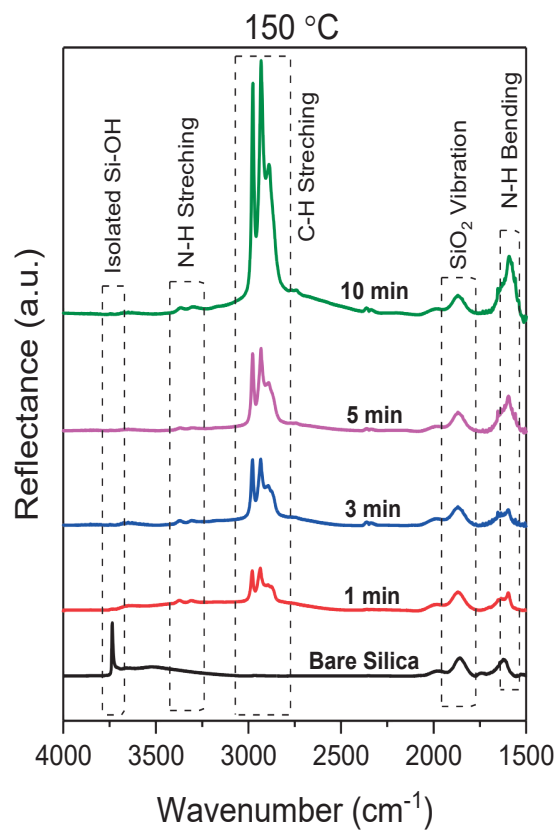

c)

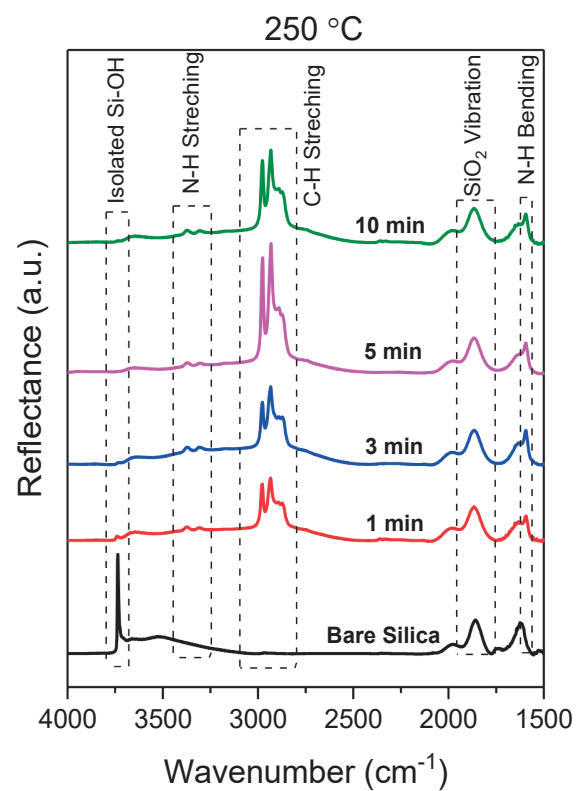

b.

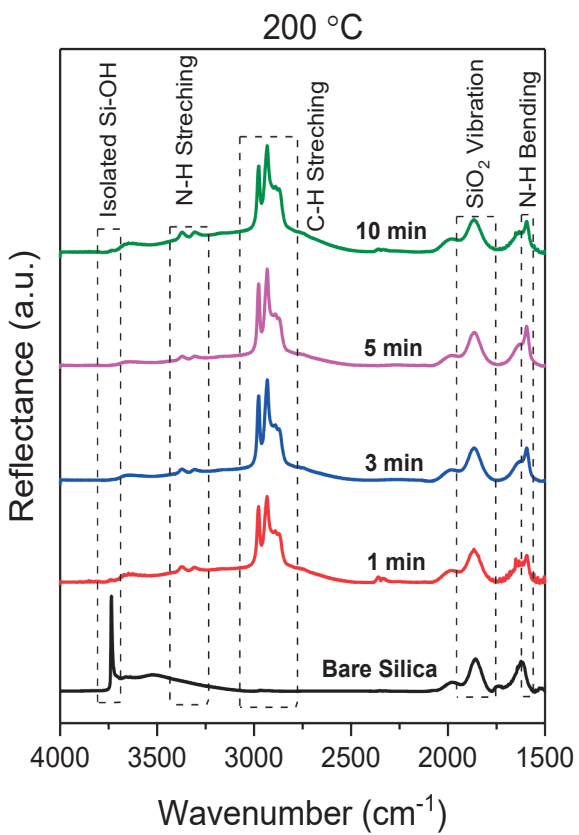

Figure 5.4 . DRIFTS spectra for all silica samples treated at different temperatures 
The APS grafting densities after 1, 3, 5 and 10 min of precursor pulse time, at three selected temperatures, were calculated based on the TGA results and using Equation 5-4. The results are plotted in Figure 5.5a. It can be seen that at $150^{\circ} \mathrm{C}$, the silanization reaction is not surface-limiting; the deposited layer keeps growing as the APTES pulse time increases. However, at $200^{\circ} \mathrm{C}$ and $250{ }^{\circ} \mathrm{C}$, the grafting density of the silane reaches saturation around $0.5 \mathrm{mmol} / \mathrm{g}$ silica approximately after 5 minutes of pulse time. This observation indicates, firstly, that at $150{ }^{\circ} \mathrm{C}$, the amount of physically and chemically adsorbed water on the silica enables for hydrolyzation of the APTES species and increasing the grafting density with time. This would cause oligomerization of the precursor, and prevents conformal deposition of the APS layer. Instead, at $200{ }^{\circ} \mathrm{C}$ and $250^{\circ} \mathrm{C}$, the absence of the physisorbed water and the lower number of remaining silanol groups prevents APTES oligomerization, resulting in surface saturation after 5 minutes. This can be an evidence that the APTES precursor has formed a sub-monolayer of APS on the silica surface preventing any further silanization. The trend for the carbon and nitrogen content is similar to that of the grafting densities estimated from TGA (Figures 5.5b and 5.5c). This confirms that the self-limiting character of this process is valid down to the atomic scale, which is another key feature of an ALD process. 
a.

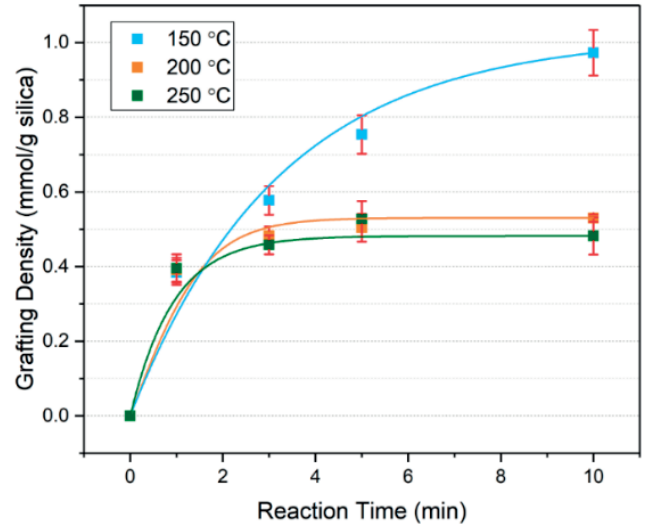

b.

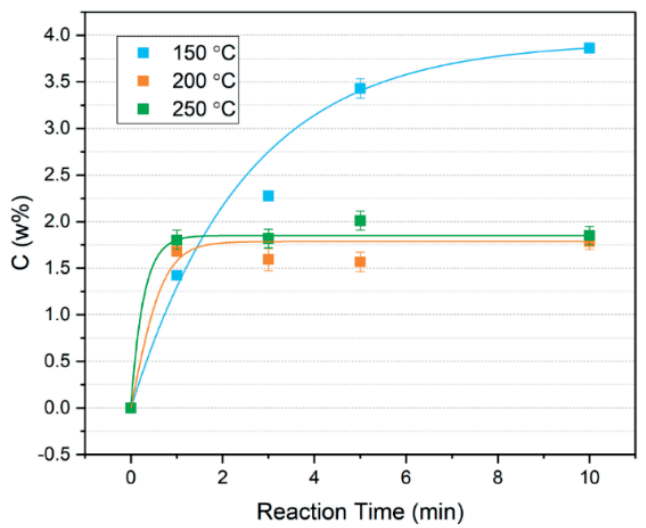

C.

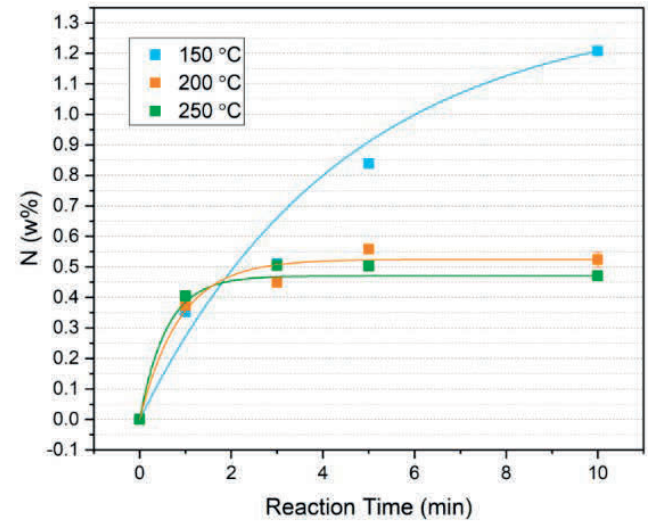

Figure 5.5. a: APS grafting densities after 1, 3, 5 and $10 \mathrm{~min}$ of precursor pulse time, at three selected temperatures, $\mathrm{b}$ : and c: carbon and nitrogen content from elemental analysis; the fitted curves are to guide the eye 
Considering an average of $4 \mathrm{SiOH} / \mathrm{nm}^{2}$ on the surface of AEROSIL 200 (Equation 5-1), and the number of silanol groups siloxanating at each reaction temperature (Equation 5-2), the saturation grafting density of APS can be estimated ( $G D_{\text {cal }}^{\text {sat }}$ in Equation 5-3) and compared to the experimental value at each reaction temperature $\left(G D_{\text {exp }}^{\text {sat }}\right.$ in Equation 5-4). The results are presented in Table 5.2. The number of reactive silanol groups decreases by increasing the reaction temperature, what is expected as it was observed in form of mass loss seen in Figure 5.2. The calculated GD upon saturation is close to the experimental value when the reaction was performed at $200{ }^{\circ} \mathrm{C}$. An average grafting of $1.54 \frac{\mathrm{APS}}{\mathrm{nm}^{2}}$, compared to $1.79 \frac{\mathrm{SiOH}}{\mathrm{nm}^{2}}$ available on the silica nanoparticles (see Table 5.2), implies the formation of an APS sub-monolayer at $200^{\circ} \mathrm{C}$ after 5 minutes. This also implies that the APTES monodentate structure is more likely to occur at $200^{\circ} \mathrm{C}$, whereas the presence of water at $150{ }^{\circ} \mathrm{C}$, and the promoted condensation at $250{ }^{\circ} \mathrm{C}$ may result more in bidentate and tridentate structures ${ }^{34}$. Accordingly, one can estimate that $86 \%$ of the silanol groups on the silica have reacted with the silane at $200{ }^{\circ} \mathrm{C}$. This is a significant coverage e.g. compared to the $25 \%$ of reacted silanols in case of silanization in silica-filled rubber compounds ${ }^{26}$. A maximum of 2.92 APS molecules are attached per $\mathrm{nm}^{2}$ of silica at $150{ }^{\circ} \mathrm{C}$, which is on average higher than the number of available $\mathrm{SiOH}$ groups on the surface at this temperature. This estimation shows that precursor has been oligomerized at this reaction temperature. Interestingly, according to these calculations, it seems that the same phenomenon has occurred in case of the reaction at $250{ }^{\circ} \mathrm{C}$. An average of $1.57 \frac{\mathrm{APS}}{\mathrm{nm}^{2}}$ is deposited after 5 minutes of exposure at this temperature, which is higher than $1.05 \frac{\mathrm{SiOH}}{\mathrm{nm}^{2}}$ available on the surface (see Table 5.2). This can be due to condensation and crosslinking of APTES species which was shown to be promoted at high reaction temperatures ${ }^{34}$. This effect would result in an increased number of APS grafted onto a unit surface area, compared to the expected value calculated from Equation 5-3. Unlike the reaction at $150{ }^{\circ} \mathrm{C}$, the APS growth mechanism at $250{ }^{\circ} \mathrm{C}$ is more affected by horizontal oligomerization of APTES. Nevertheless, despite the condensation effect, the deposition at $250{ }^{\circ} \mathrm{C}$ reaches saturation, as the number of silanol groups on the silica is relatively low at such a high temperature. 
Table 5.2. Number of silanol groups and the calculated and experimental values of APS grafting density at three reaction temperatures

\begin{tabular}{cccccc}
\hline $\begin{array}{c}\text { Reaction } \\
\text { Temperature }\end{array}$ & $n_{\text {SiOH }}^{T}\left(\frac{\text { SiOH }}{n m^{2}}\right)$ & $G D_{\text {cal }}^{\text {sat }}\left(\frac{m \text { mol APS }}{\text { g Silica }}\right)$ & $\begin{array}{c}\text { TGA Mass } \\
\text { Loss }(\%)\end{array}$ & $G D_{\text {exp }}^{\text {sat }}\left(\frac{m m o l \text { APS }}{g \text { Silica }}\right)$ & $G D_{\text {exp }}^{\text {sat }}\left(\frac{A P S}{n m^{2}}\right)$ \\
$150{ }^{\circ} \mathrm{C}$ & $2.56 \pm 0.07$ & $0.85 \pm 0.02$ & $5.2 \pm 0.8$ & $0.97 \pm 0.03$ & $2.92 \pm 0.09^{*}$ \\
$200{ }^{\circ} \mathrm{C}$ & $1.79 \pm 0.03$ & $0.59 \pm 0.01$ & $3.6 \pm 0.2$ & $0.51 \pm 0.02$ & $1.54 \pm 0.06$ \\
$250{ }^{\circ} \mathrm{C}$ & $1.05 \pm 0.03$ & $0.35 \pm 0.01$ & $3.8 \pm 0.3$ & $0.52 \pm 0.02$ & $1.57 \pm 0.06$
\end{tabular}

${ }^{*}$ Since saturation was not achieved at $150{ }^{\circ} \mathrm{C}$, the maximum achieved GD (10 min pulse time) is reported here.

Due to the strong van der Waals forces between silica nanoparticles, agglomeration in a fluidized bed is inevitable ${ }^{35}$. In fact, in contrast to particles in the high micrometer range, nanoparticles are not fluidized individually but as agglomerates: very dilute clusters in the order of $100 \mu \mathrm{m}$ consisting of billions of primary particles. However, the high van der Waals forces can cause the nanoparticles to form larger agglomerates at the bottom of the fluidized bed column. Grillo et al. ${ }^{36}$ demonstrated that despite the agglomerated structure of nanoparticles, surface saturation is feasible even at ambient conditions, which indicates the effective diffusion of the precursor into agglomerates in fluidized bed ALD processes. In our case, large agglomerates, up to $5 \mathrm{~mm}$ in diameter, were observed at the bottom of the reactor in all the experiments. Accordingly, it is fair to ask the following question: Is the APS deposition conformal, both in the fine fluidized powder and the agglomerates at the bottom of the reactor? Figure 5.6 shows the TGA graphs for fine and agglomerated powders of all the samples treated at different temperatures and times. In case of reaction temperatures of $200^{\circ} \mathrm{C}$ and $250{ }^{\circ} \mathrm{C}$, the graphs of the fine and agglomerated silica are nearly identical. This indicates that the gas phase deposition of APS is fairly conformal at the temperatures at which surface-limiting behavior takes place. This is another key factor of an ALD process, in which any exposed particle surface with the ability to bond functional groups can be coated, including internal surfaces of porous substrates or agglomerates of nanoparticles ${ }^{37}$. At $150{ }^{\circ} \mathrm{C}$, there is a significant difference between the fine powders and the large agglomerates indicating a non-conformal deposition of APS at this temperature. The fine powder, where primary particles are fully exposed to the gaseous precursors, show higher 
APTES loadings at this temperature. Whereas, very large non-fluidized agglomerates may suffer from diffusion limitations of the gaseous precursors, thereby preventing a uniform and conformal deposition on each primary particle ${ }^{31}$. It can be seen that this difference tends to be even greater as the reaction time increases, implying an increased inhomogeneity in the deposited layer with time.

$T_{\text {reaction }}=150^{\circ} \mathrm{C}$
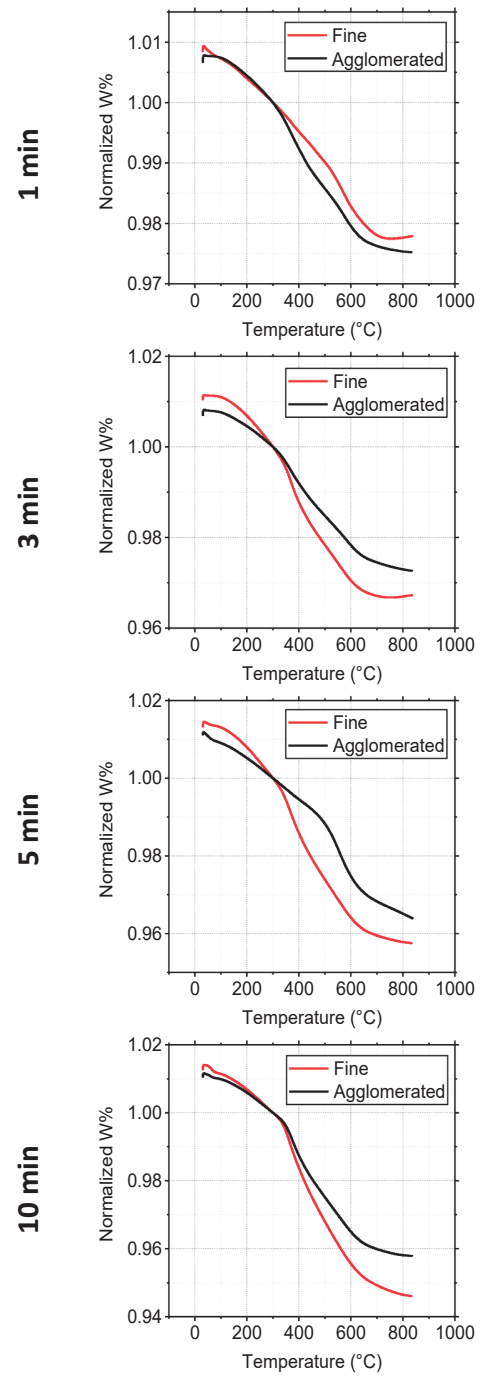

$\mathrm{T}_{\text {reaction }}=200^{\circ} \mathrm{C}$
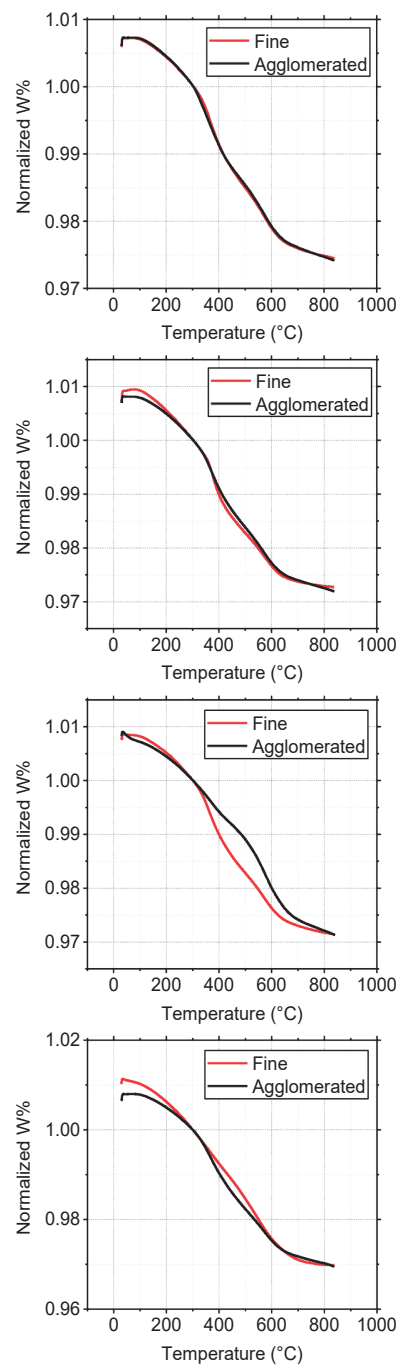

$T_{\text {reaction }}=250^{\circ} \mathrm{C}$
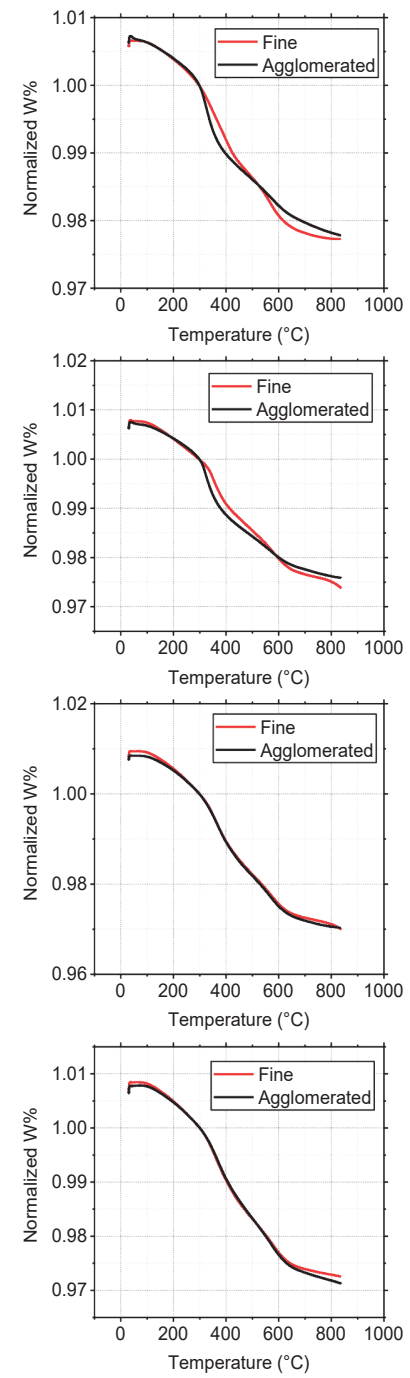

Figure 5.6. TGA graphs for fine and agglomerated powders of all treated silica samples at different temperatures and reaction times 


\subsection{Repetition of Amino-Silane/Water Cycles}

The above results showed that self-limiting APS layers can be deposited onto fluidized silica nanoparticles at $200{ }^{\circ} \mathrm{C}$, reaching a grafting density of ca. $0.5 \mathrm{mmol} / \mathrm{g}$ silica after 5 minutes. The saturation occurs inasmuch as there are no hydrolyzed ethoxy groups accessible anymore at a certain point for the unreacted silane to engage with. Therefore, it is expected that sequential APTES/water pulses through the fluidized silica nanoparticles would result in further growth of the deposited layer upon each cycle.

The APTES grafting density is calculated from TGA data and plotted against the number of APTES/water cycles in Figure 5.7a. It is clear that increasing the number of cycles results in higher grafting densities of APTES. This confirms that the mechanism shown in Figure $5.1 \mathrm{~b}$ is valid for the controlled deposition of the silane: by choosing the appropriate number of cycles, it is possible to reach the desired grafting densities. The above trend is also observed at atomic level as the elemental analysis results show in Figures 5.7b and 5.7c: It is perfectly clear that the $\mathrm{C}$ and $\mathrm{N}$ content follow the same trend as the grafting density does. The $\mathrm{C}: \mathrm{N}$ ratio decreases by increasing the number of cycles, as it is evident in Figure 5.7d. The C:N ratio for the unreacted APTES is 9, whereas ideal APS films can be characterized by a C:N ratio of 3, indicative of complete hydrolysis of APTES molecules, and replacement of all ethoxy groups with siloxane bonds. The C:N approaching 3 upon increasing cycles is indicative of a more complete hydrolysis of the precursor. 
a.

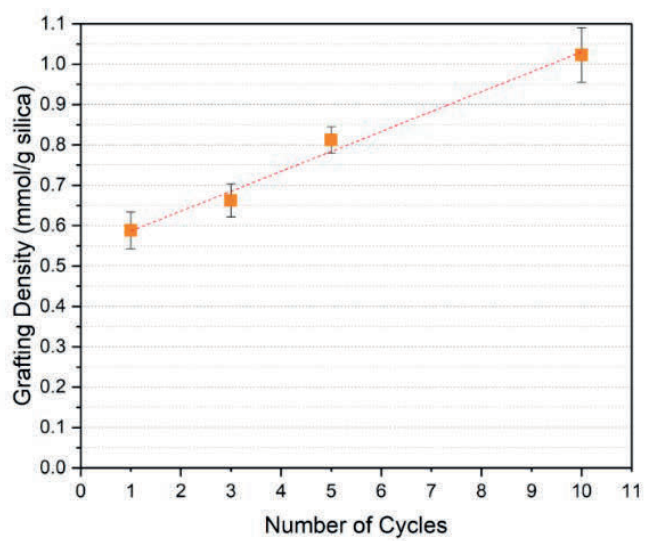

c.

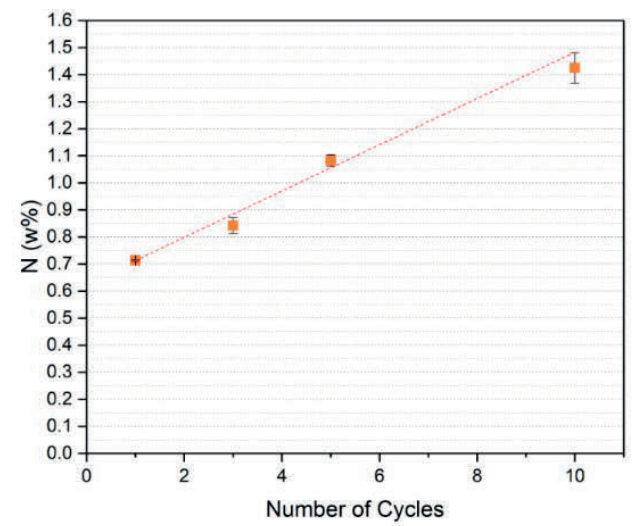

b.

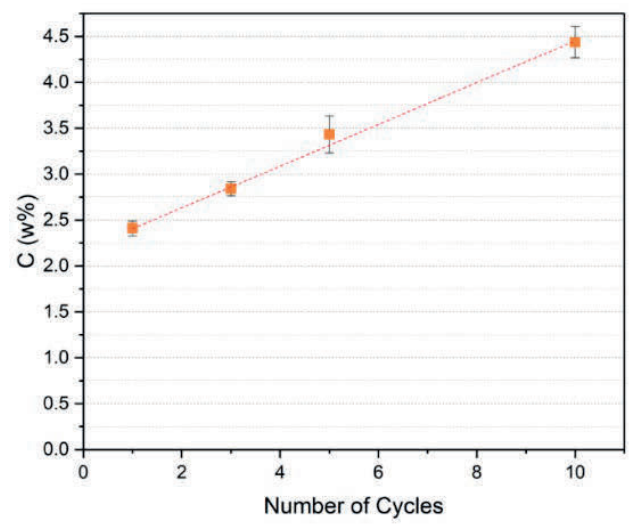

d.

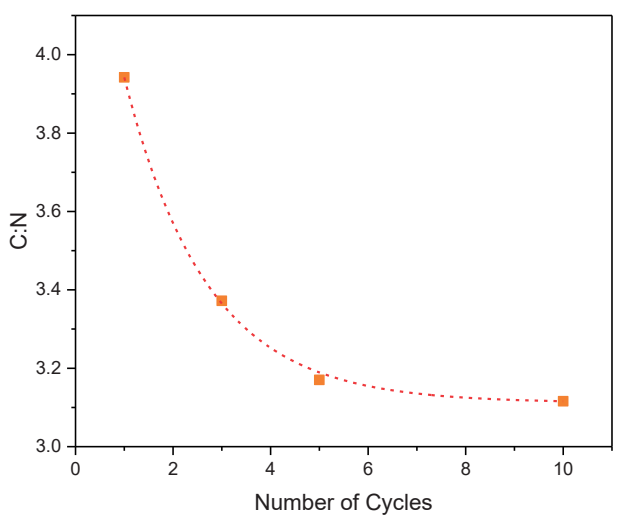

Figure 5.7. a: APS grafting densities after $1,3,5$ and 10 APTES/water cycles, at $200^{\circ} \mathrm{C}$, b: carbon, c: nitrogen content and $\mathrm{d}$ : $\mathrm{C}: \mathrm{N}$ ratio from elemental analysis

The XPS spectra of silica samples modified by 10 APTES/water cycles are presented in Figure 5.8a along with the one of the untreated silica for comparison. The emergence of the peaks corresponding to $\mathrm{C} 1 \mathrm{~s}$ and $\mathrm{N} 1 \mathrm{~s}$ in the modified silica confirms the presence of APTES on the surface. The deconvoluted elemental fine scans in Figures 5.8b and 5.8c indicate the atomic environment and are dependent on the chemical bonds in the film. As expected, the $\mathrm{N} 1 \mathrm{~s}$ fine scan reveals that there are two peaks at $399.6 \mathrm{eV}$ and $400.7 \mathrm{eV}$, indicative of C-N and $\mathrm{N}-\mathrm{H}$ bonds, respectively ${ }^{38-39}$. The $\mathrm{C} 1 \mathrm{~s}$ scan also exhibits two peaks, one at $285 \mathrm{eV}$ representing $\mathrm{C}-\mathrm{C}$ and $\mathrm{C}-\mathrm{H}$ and at $286.1 \mathrm{eV}$ corresponding to $\mathrm{C}-\mathrm{N}$ bonds 38,40 . 
a.

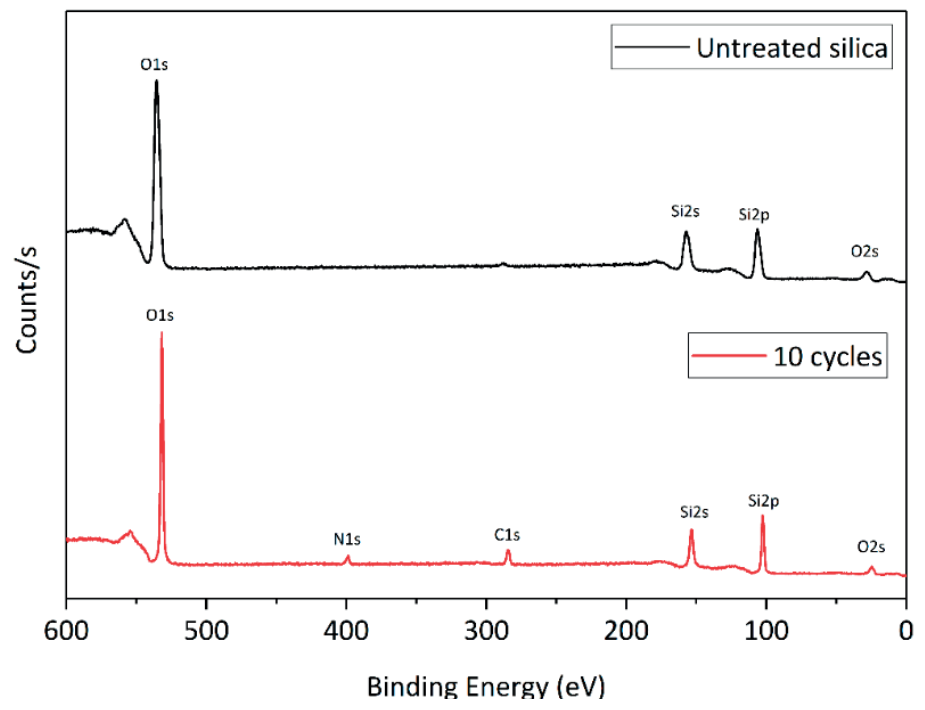

b.

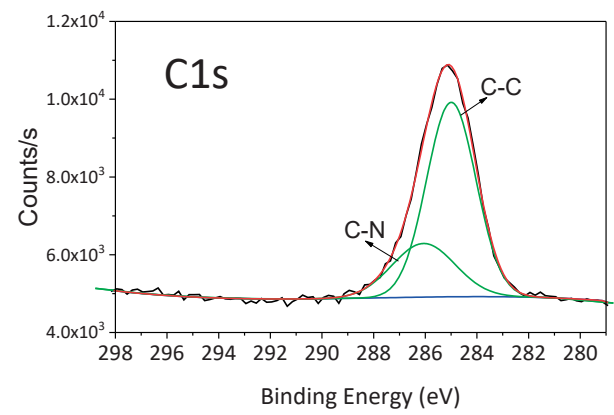

C.

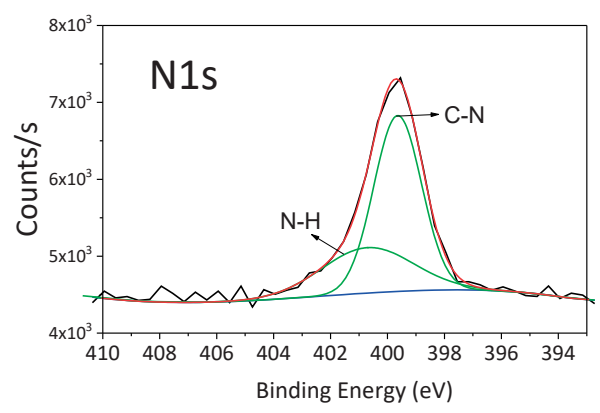

Figure 5.8. a: XPS full spectra of the silica sample treated in 10 cycles compared to the untreated reference, $b$ and $c$ : the deconvoluted $\mathrm{C} 1 \mathrm{~s}$ and $\mathrm{N} 1 \mathrm{~s}$ elemental fine scans for the silica sample treated in 10 cycles

The atomic percentages of $\mathrm{C}$ and $\mathrm{N}$ as well as the $\mathrm{C}: \mathrm{N}$ ratio of the silica modified in cycles are presented in Table 5.3, along with the modified silica reaching saturation after 5 minutes of APTES pulse at $200{ }^{\circ} \mathrm{C}$. It is clear that both, the carbon and the nitrogen atom content, increases on the surface of the nanoparticles upon increasing the number of cycles. While this is expected according to the previous test results, the increase in the carbon content should be less significant due to the hydrolysis and release of the ethoxy groups in 
form of ethanol. This effect is manifested in the decrease in the $\mathrm{C}: \mathrm{N}$ ratios which was also observed in Figure 5.7d. The fact that the C:N ratio is closing down to 3 upon increasing the number of cycles (Figure 5.9), indicates that a conformal APS layer has been formed on the silica nanoparticles, and crosslinked via horizontal siloxanation.

Table 5.3. Atomic percentages of $\mathrm{C}$ and $\mathrm{N}$ from XPS, along with $\mathrm{C}: \mathrm{N}$ ratios

\begin{tabular}{cccc}
\hline Silica Sample & C (at\%) & N (at\%) & C:N \\
\hline Saturated w/o hydrolyzation & 5.35 & 1.32 & 4.05 \\
1 Cycle & 7.63 & 1.94 & 3.93 \\
3 Cycles & 8.33 & 2.24 & 3.72 \\
5 Cycles & 9.13 & 2.51 & 3.54 \\
10 Cycles & 11.34 & 3.43 & 3.31
\end{tabular}

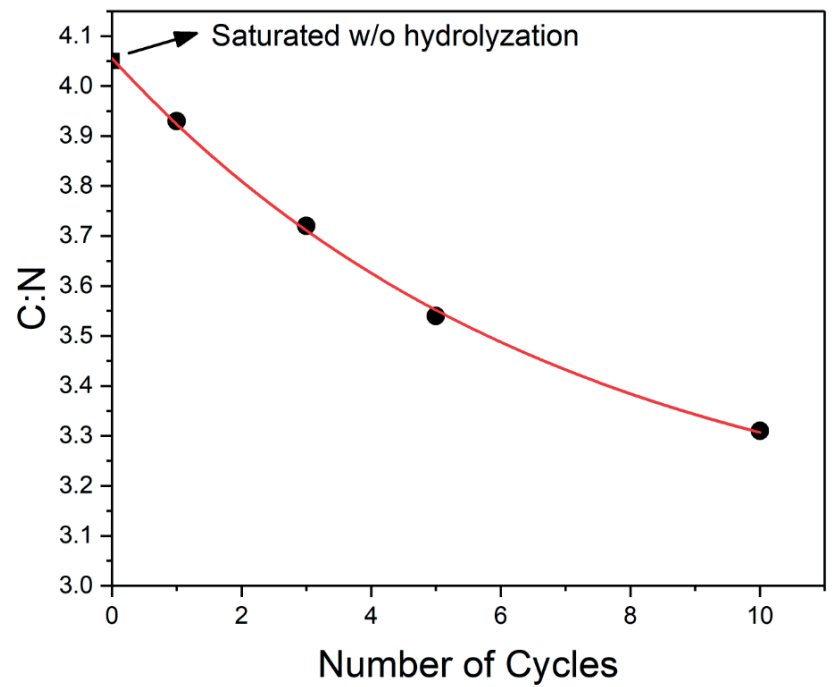

Figure 5.9. C:N ratio of the silica modified in cycles 


\section{Conclusions}

Controlled deposition of APTES was performed on fumed silica nanoparticles in a fluidized bed. A self-limiting surface saturation, similar to an ALD process, was achieved when the reaction took place at $200{ }^{\circ} \mathrm{C}$. The surface saturation occurring at this temperature was due to the absence of released water from the silica during the process, thus preventing oligomerization of the precursor. With this self-limiting feature, and using water as coreactant, it was possible to hydrolyze the unreacted ethoxy groups in the deposited layer, and hence to chemically graft multiple layers of aminopropylsiloxane onto silica when sequential APTES/water cycles were performed on the fluidized nanoparticles. In this way, it was possible to reach a more complete coating. Increasing the number of cycles resulted in increasing the thickness of the deposited layer enabling for a controlled engineering of the surface of the functionalized nanoparticles.

\section{References}

[1] Mahtabani, A.; Alimardani, M.; Razzaghi-Kashani, M., Further evidence of fillerfiller mechanical engagement in rubber compounds filled with silica treated by long-chain silane. Rubber Chemistry and Technology, 90 (3), 2017, pp 508-520.

[2] He, X.; Rytöluoto, I.; Anyszka, R.; Mahtabani, A.; Saarimäki, E.; Lahti, K.; Paajanen, M.; Dierkes, W.; Blume, A., Surface Modification of Fumed Silica by Plasma Polymerization of Acetylene for PP/POE Blends Dielectric Nanocomposites. Polymers, 11 (12), 2019, p 1957. [3] Mathabani, A.; Rytöluoto, I.; He, X.; Saarimäki, E.; Lahti, K.; Paajanen, M.; Anyszka, R.; Dierkes, W.; Blume, A. Solution Modified Fumed Silica and Its Effect on Charge Trapping Behavior of PP/POE/Silica Nanodielectrics, Proceedings of the Nordic Insulation Symposium, 2019; pp 129-133.

[4] Wang, X.; Lin, K. S. K.; Chan, J. C. C.; Cheng, S., Direct Synthesis and Catalytic Applications of Ordered Large Pore Aminopropyl-Functionalized SBA-15 Mesoporous Materials. The Journal of Physical Chemistry B, 109 (5), 2005, pp 1763-1769.

[5] Li, Y.; Li, N.; Pan, W.; Yu, Z.; Yang, L.; Tang, B., Hollow Mesoporous Silica Nanoparticles with Tunable Structures for Controlled Drug Delivery. ACS Applied Materials \& Interfaces, 9 (3), 2017, pp 2123-2129.

[6] Protsak, I. S.; Morozov, Y. M.; Dong, W.; Le, Z.; Zhang, D.; Henderson, I. M., A 29Si, $1 \mathrm{H}$, and $13 \mathrm{C}$ Solid-State NMR Study on the Surface Species of Various Depolymerized Organosiloxanes at Silica Surface. Nanoscale Research Letters, 14 (1), 2019, p 160.

[7] Protsak, I.; Henderson, I. M.; Tertykh, V.; Dong, W.; Le, Z.-C., Cleavage of Organosiloxanes with Dimethyl Carbonate: A Mild Approach To Graft-to-Surface Modification. Langmuir, 34 (33), 2018, pp 9719-9730. 
[8] Zhang, L.; Xiong, Y.; Ou, E.; Chen, Z.; Xiong, Y.; Xu, W., Preparation and properties of nylon 6/carboxylic silica nanocomposites via in situ polymerization. Journal of Applied Polymer Science, 122 (2), 2011, pp 1316-1324.

[9] Oh, S.; Kang, T.; Kim, H.; Moon, J.; Hong, S.; Yi, J., Preparation of novel ceramic membranes modified by mesoporous silica with 3-aminopropyltriethoxysilane (APTES) and its application to $\mathrm{Cu} 2+$ separation in the aqueous phase. Journal of Membrane Science, 301 (1), 2007, pp 118-125.

[10] Kłonkowski, A. M.; Grobelna, B.; Widernik, T.; Jankowska-Frydel, A.; Mozgawapar, W., Coordination state of copper(II) complexes anchored and grafted onto the surface of organically modified silicates. Langmuir, 15 (18), 1999, pp 5814-5819.

[11] Jal, P. K.; Patel, S.; Mishra, B. K., Chemical modification of silica surface by immobilization of functional groups for extractive concentration of metal ions. Talanta, 62 (5), 2004, pp 1005-1028.

[12] Kim, J.; Cho, J.; Seidler, P. M.; Kurland, N. E.; Yadavalli, V. K., Investigations of Chemical Modifications of Amino-Terminated Organic Films on Silicon Substrates and Controlled Protein Immobilization. Langmuir, 26 (4), 2010, pp 2599-2608.

[13] Briand, E.; Humblot, V.; Landoulsi, J.; Petronis, S.; Pradier, C.-M.; Kasemo, B.; Svedhem, S., Chemical Modifications of $\mathrm{Au} / \mathrm{SiO} 2$ Template Substrates for Patterned Biofunctional Surfaces. Langmuir, 27 (2), 2011, pp 678-685.

[14] Mahtabani, A.; Rytöluoto, I.; Anyszka, R.; He, X.; Saarimäki, E.; Lahti, K.; Paajanen, M.; Dierkes, W.; Blume, A., On the Silica Surface Modification and Its Effect on Charge Trapping and Transport in PP-Based Dielectric Nanocomposites. ACS Applied Polymer Materials, 2 (8), 2020, pp 3148-3160.

[15] Chang, K.-C.; Lin, C.-Y.; Lin, H.-F.; Chiou, S.-C.; Huang, W.-C.; Yeh, J.-M.; Yang, J.-C., Thermally and mechanically enhanced epoxy resin-silica hybrid materials containing primary amine-modified silica nanoparticles. Journal of Applied Polymer Science, 108 (3), 2008, pp 1629-1635.

[16] Liu, D.; Hoang, A. T.; Pourrahimi, A. M.; Pallon, L. K. H.; Nilsson, F.; Gubanski, S. M.; Olsson, R. T.; Hedenqvist, M. S.; Gedde, U. W., Influence of nanoparticle surface coating on electrical conductivity of LDPE/AI2O3 nanocomposites for HVDC cable insulations. IEEE Transactions on Dielectrics and Electrical Insulation, 24 (3), 2017, pp 1396-1404.

[17] Liu, D.; Pourrahimi, A. M.; Pallon, L. K.; Sánchez, C. C.; Olsson, R. T.; Hedenqvist, M. S.; Fogelström, L.; Malmström, E.; Gedde, U. W., Interactions between a phenolic antioxidant, moisture, peroxide and crosslinking by-products with metal oxide nanoparticles in branched polyethylene. Polymer Degradation and Stability, 125, 2016, pp 21-32.

[18] Nilsson, F.; Karlsson, M.; Pallon, L.; Giacinti, M.; Olsson, R. T.; Venturi, D.; Gedde, U. W.; Hedenqvist, M. S., Influence of water uptake on the electrical DC-conductivity of insulating LDPE/MgO nanocomposites. Composites Science and Technology, 152, 2017, pp 11-19.

[19] Vansant, E. F.; Van Der Voort, P.; Vrancken, K. C., Characterization and chemical modification of the silica surface Elsevier: 1995; Vol. 93.

[20] Qiao, B.; Wang, T.-J.; Gao, H.; Jin, Y., High density silanization of nano-silica particles using $\gamma$-aminopropyltriethoxysilane (APTES). Applied Surface Science, 351, 2015, pp 646-654. 
[21] Lazghab, M.; Saleh, K.; Guigon, P., Functionalisation of porous silica powders in a fluidised-bed reactor with glycidoxypropyltrimethoxysilane (GPTMS) and aminopropyltriethoxysilane (APTES). Chemical engineering research and design, 88 (5-6), 2010, pp 686-692.

[22] Krasnoslobodtsev, A. V.; Smirnov, S. N., Effect of water on silanization of silica by trimethoxysilanes. Langmuir, 18 (8), 2002, pp 3181-3184.

[23] Moon, J. H.; Shin, J. W.; Kim, S. Y.; Park, J. W., Formation of uniform aminosilane thin layers: an imine formation to measure relative surface density of the amine group. Langmuir, 12 (20), 1996, pp 4621-4624.

[24] Lazghab, M.; Saleh, K.; Guigon, P., A new solventless process to hydrophobize silica powders in fluidized beds. AlChE journal, 54 (4), 2008, pp 897-908.

[25] Ek, S.; liskola, E. I.; Niinistö, L.; Vaittinen, J.; Pakkanen, T. T.; Keränen, J.; Auroux, A., Atomic layer deposition of a high-density aminopropylsiloxane network on silica through sequential reactions of $\gamma$-aminopropyltrialkoxysilanes and water. Langmuir, 19 (25), 2003, pp 10601-10609.

[26] Blume, A.; Jin, J.; Mahtabani, A.; He, X.; Kim, S.; Andrzejewska, Z. New Structure Proposal for Silane Modified Silica, International Rubber Conference, IRC 2019, London, 2019.

[27] Ek, S.; Root, A.; Peussa, M.; Niinistö, L., Determination of the hydroxyl group content in silica by thermogravimetry and a comparison with $1 \mathrm{H}$ MAS NMR results. Thermochimica Acta, 379 (1), 2001, pp 201-212.

[28] Morrow, B. A.; McFarlan, A. J., Infrared and gravimetric study of an aerosil and a precipitated silica using chemical and hydrogen/deuterium exchange probes. Langmuir, 7 (8), 1991, pp 1695-1701.

[29] Mueller, R.; Kammler, H. K.; Wegner, K.; Pratsinis, S. E., OH Surface Density of SiO2 and TiO2 by Thermogravimetric Analysis. Langmuir, 19 (1), 2003, pp 160-165.

[30] Wikström, P.; Mandenius, C. F.; Larsson, P.-o., Gas phase silylation, a rapid method for preparation of high-performance liquid chromatography supports. Journal of Chromatography A, 455, 1988, pp 105-117.

[31] Zhang, D.; La Zara, D.; Quayle, M. J.; Petersson, G.; van Ommen, J. R.; Folestad, S., Nanoengineering of crystal and amorphous surfaces of pharmaceutical particles for biomedical applications. ACS Applied Bio Materials, 2 (4), 2019, pp 1518-1530.

[32] Grillo, F.; La Zara, D.; Mulder, P.; Kreutzer, M. T.; Ruud van Ommen, J., Oriented Attachment and Nanorod Formation in Atomic Layer Deposition of TiO2 on Graphene Nanoplatelets. The Journal of Physical Chemistry C, 122 (34), 2018, pp 19981-19991.

[33] Gorski, D.; Klemm, E.; Fink, P.; Hörhold, H.-H., Investigation of quantitative SiOH determination by the silane treatment of disperse silica. Journal of Colloid and Interface Science, 126 (2), 1988, pp 445-449.

[34] Yang, S.-q.; Yuan, P.; He, H.-p.; Qin, Z.-h.; Zhou, Q.; Zhu, J.-x.; Liu, D., Effect of reaction temperature on grafting of $\gamma$-aminopropyl triethoxysilane (APTES) onto kaolinite. Applied Clay Science, 62-63, 2012, pp 8-14.

[35] van Ommen, J. R.; Valverde, J. M.; Pfeffer, R., Fluidization of nanopowders: a review. Journal of Nanoparticle Research, 14 (3), 2012, p 737. 
[36] Grillo, F.; Kreutzer, M. T.; van Ommen, J. R., Modeling the precursor utilization in atomic layer deposition on nanostructured materials in fluidized bed reactors. Chemical Engineering Journal, 268, 2015, pp 384-398.

[37] King, D. M.; Liang, X.; Weimer, A. W., Functionalization of fine particles using atomic and molecular layer deposition. Powder Technology, 221, 2012, pp 13-25.

[38] Yan, X.; Xu, T.; Chen, G.; Yang, S.; Liu, H.; Xue, Q., Preparation and characterization of electrochemically deposited carbon nitride films on silicon substrate. Journal of Physics D: Applied Physics, 37 (6), 2004, pp 907-913.

[39] Dementjev, A. P.; de Graaf, A.; van de Sanden, M. C. M.; Maslakov, K. I.; Naumkin, A. V.; Serov, A. A., X-Ray photoelectron spectroscopy reference data for identification of the C3N4 phase in carbon-nitrogen films. Diamond and Related Materials, 9 (11), 2000, pp 1904-1907.

[40] Endo, K.; Maeda, S.; Aida, M., Simulation of C1s Spectra of C- and O-Containing Polymers in XPS by ab initio MO Calculations Using Model Oligomers. Polymer Journal, 29 (2), 1997, pp 171-181. 



\section{CHAPTER 6}

Gas-Phase Modified Silica Nanoparticles in Polypropylene-Based Nanodielectrics 


\begin{abstract}
Dielectric nanocomposites have shown considerable potential for high performance insulation applications. Tailoring the large interfacial areas induced by the nanoparticles is of great importance when developing insulating nanodielectrics. The introduction of electrically active functional groups to the surface of nanoparticles can create localized states at the interface, and alter the charge carrier transport and space charge formation within the nanocomposite (NC). Therefore, implementing changes in the electronic structure of the filler surface upon its functionalization can provide information about the alterations in macroscopic dielectric properties of the NC. In the present study, the electronic structure of silica nanoparticles modified with 3-aminopropyltriethoxysilane (APTES) is demonstrated using density functional theory (DFT) calculations. In order to confirm the DFT predictions, fumed silica nanoparticles are modified via a self-limiting process, similar to atomic layer deposition (ALD). Subsequently, the dielectric properties of NCs based on the modified silica and a polypropylene (PP) / ethylene-octene-copolymer (EOC) blend are investigated, and the effect of the amino functional groups on charge trapping and transport through the NCs is discussed. With a good correlation between DFT calculations and experimental data, we demonstrate that the APTES modified silica exhibits new localized states, a large density of valence states and a certain electron/hole distribution that can suppress the formation space charge at the filler-polymer interfaces.
\end{abstract}




\section{Introduction}

So far, it has been shown that the presence of deep carrier traps at the interfaces can be beneficial for insulating nanocomposites (NCs), as they can suppress the formation of space charge under DC electric fields. Furthermore, deep traps can capture mobile carriers ultimately responsible for breakdown ${ }^{1}$ and alter the distribution of space charge ${ }^{2}$. By introducing electrically active moieties to the nanofiller surface, it is possible to engineer the filler-polymer interfaces, and alter charge carrier trapping and transport under high electric fields ${ }^{3-5}$. In Chapter 3, 3-aminopropyltriethoxysilane (APTES) was used to modify the surface of silica NPs in solution. The presence of the amine functional groups induced deep trap states (with energy levels generally above $1 \mathrm{eV}$ ) that suppressed the injection of space charge and reduced the overall charge carrier mobility in the studied polypropylene (PP) based nanodielectrics ${ }^{6}$. These observations, and similar observations in other studies ${ }^{7-}$ ${ }^{9}$, have led to the hypothesis that the amine functional groups may alter the electronic structure of the silica, resulting in such behavior. In order to understand the role of this polar functionality in alteration of charge trapping and space charge formation in NCs, it is crucial to have a closer look at the electronic properties of the silica surface, before and after modification. Density Functional Theory (DFT) is an accurate, efficient fundamental method for numerical computation of electronic, atomic and molecular structure of matter, by solvation of the time-independent Schrödinger's equation ${ }^{10}$. With the use of DFT, it is possible to calculate not only the ground state geometry of the studied model, but also the density of states (DOS) in the vicinity of the band-gap, related to the highest occupied molecular orbitals (HOMO), lowest unoccupied molecular orbitals (LUMO) and electron/hole trapping states in that structure. Nevertheless, to confidently confirm calculation results with experimental data, an accurate repeatable method for NP modification is needed.

Atomic layer deposition (ALD) is a promising method to deposit conformal homogenous thin films onto organic and inorganic substrates, including NPs ${ }^{11-14}$. ALD, relying on gas-solid surface reactions, can potentially address the common shortcomings of conventional solution methods, and enables outstanding control over the amount of 
deposited material on the NPs ${ }^{15-16}$. ALD offers self-limiting layer-by-layer growth of the deposited material, as opposed to other thin film techniques, such as chemical vapor deposition (CVD), which relies on simultaneous reaction of two precursors with the reactive surface ${ }^{17}$. In the previous chapter, we demonstrated successful deposition of aminopropylsiloxane (APS) layers on fluidized silica NPs through sequential pulses of APTES and water as precursors. The self-limiting reaction observed in this process was similar to ALD processes, and allowed to deposit APS films on NPs in a controlled layer-by-layer fashion.

In this chapter, firstly we demonstrate the electronic structure of APTES modified silica using density functional theory (DFT) results. The ground state geometry of the APTES-silica structure is presented, and its band structure is compared to the unmodified silica under an external electric field. To confirm DFT results experimentally, NCs based on a polypropylene (PP)/ethylene-octene-copolymer (EOC) blend are prepared, containing unmodified and APTES-modified silica NPs. The effect of the amine functional group on charge trapping and transport in the aforementioned NCs is investigated using thermally stimulated depolarization current (TSDC) measurements. In addition, to further analyze the dielectric response of the NCs with respect to the frequency of the applied field, broadband dielectric spectroscopy (BDS) is utilized. The morphological and crystalline structure of the studied NCs measured by differential scanning calorimetry (DSC) and scanning electron microscopy (SEM) techniques are discussed.

\section{Experimental Work}

\subsection{Silica Modification and Characterization}

Silica (AEROSIL 200, Evonik Ind., Germany) treatment was carried out in a fluidized bed reactor according to what was described in Chapter 5 . We demonstrated that after 5 minutes of APTES pulse time with a reaction temperature of $200{ }^{\circ} \mathrm{C}$, the surface of silica NPS would be covered by a sub-monolayer of grafted APTES, with no reactive $-\mathrm{OH}$ groups accessible to the precursor, and thus, leading to surface saturation. Using water as the second precursor, it was possible to hydrolyze the unreacted ethoxy groups in the film, and 
deposit consecutive layers of aminopropylsiloxane (APS) by pulsing 1, 3, 5 and 10 APTES $/ \mathrm{H}_{2} \mathrm{O}$ cycles through the fluidized bed, in separate experiments. The NPs were also treated at $150{ }^{\circ} \mathrm{C}$ in a non-self-limiting manner with only one pulse of APTES for 5 and 10 minutes. This was done to evaluate the effect of this self-limiting deposition on the properties of the polymer/filler interface, and its influence on the dielectric properties of NCs. Thermogravimetric Analysis (TGA) was performed on the NPs using a TGA 550 (TA Instruments, USA). The measurements were done by heating 2-3 mg of the fine powder from room temperature to 850 with a rate of $20{ }^{\circ} \mathrm{C} / \mathrm{min}$ under air atmosphere, while monitoring the mass of the sample.

Diffuse Reflectance IR Fourier Transform Spectroscopy (DRIFTS) was performed as to the description given in Chapter $3 .$.

\subsection{Preparation of the Nanocomposites}

NC samples were prepared according to the procedure and equipment described in Chapter 3.

\subsection{Morphology and Crystallinity Analysis}

Scanning Electron Microscopy (SEM) and the subsequent image analysis were performed as described in Chapter 4. No gold sputtering was utilized for SEM sample preparation. Differential Scanning Calorimetry (DSC) was performed in accordance to the description in Chapter 3.

\subsection{Thermally Stimulated Depolarization Current (TSDC)}

Details of the Thermally Stimulated Depolarization Current (TSDC) measurements were given in Chapter 3.

\subsection{Conductivity}

The DC conductivity tests were performed on selected NCs. Injection molded NC samples were pressed using a Wickert compression molding machine (Germany) at $160^{\circ} \mathrm{C}$ for 5 minutes to obtain films with thickness of $\sim 120 \mu \mathrm{m}$. Measurements were done under an electric field of $3 \mathrm{kV} / \mathrm{mm}$ applied at $70{ }^{\circ} \mathrm{C}$ with polarization time of 70 hours. The 
equipment comprised of a high voltage DC source (FUG HCN 35 - 35000, Germany), a Keysight B2980A (USA) electrometer and a custom-built measurement cell.

\subsection{Broadband Dielectric Spectroscopy (BDS)}

These measurements were performed according to what was described in Chapter 3.

\subsection{Computational Details}

Density Functional Theory (DFT) calculations were carried out on three model structures (silica, APTES and APTES-grafted silica). The models were prepared using GaussView 6, and the quantum mechanical calculations were done by the Gaussian 09W package ${ }^{18}$. The ground state geometry of each model at its minimum potential energy, depicted in Figure 6.1, was calculated utilizing the hybrid Becke three-parameter exchange correlation functional (B3LYP), with a split-valence double zeta basis set $6-31+G(d)$ to include polarization functions and long range interactions ${ }^{19-21}$. All calculations were performed considering room temperature, and in the presence of an external electric field of 0.0001 au ( $51.4 \mathrm{kV} / \mathrm{mm}$ ). Total density of states (TDOS) and the band structure were calculated for each model using the multifunctional wavefunction analyzer Multiwfn ${ }^{22}$.

The optimized $\mathrm{SiO}_{2}$ structure (Figure 6.1a), is a silica protoparticle with $\mathrm{Si}-\mathrm{O}$ bond lengths ranging between $1.58 \AA$ and $1.74 \AA$, which is in accordance with other studies ${ }^{23-25}$. Protoparticles are molecular clusters of silicon dioxide containing less than 10 to several thousands of silicon atoms, which then form primary particles through hydroxylation ${ }^{26}$. It should be noted that silica protoparticles have non-uniform structures, with a wide distribution of structural and electronic parameters that may differ from those of the particles. However, it has been shown ${ }^{27}$ that the distribution of such parameters for a set of simulated protoparticles lies within statistical range and is comparable to real particles. This would allow to translate their properties to the real situation. It is also noteworthy that the reaction of a geminal $\mathrm{Si}-\mathrm{OH}$ to the silane is generally less likely than that of an isolated one ${ }^{28}$. Nevertheless, a geminal silanol group is considered for this model so that the effect 
of an unreacted silanol group is also taken into consideration regarding the electronic states.

a.

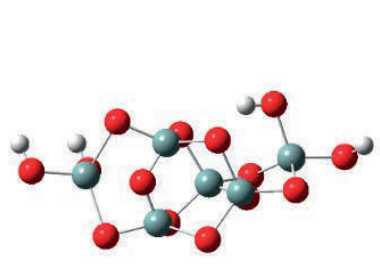

b.

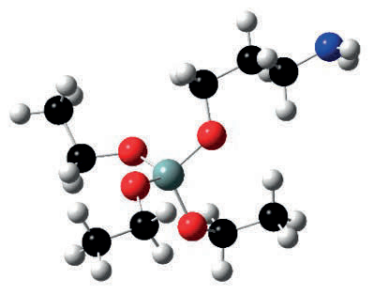

C.

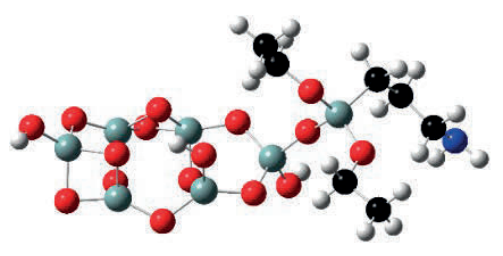

Figure 6.1. Three model structures considered for the quantum mechanical calculations: a: silica with silanol groups on both sides, b: APTES and c: APTES grafted silica with a germinal silanol group. (gray: silicon, red: oxygen, black: carbon, blue: nitrogen and white: hydrogen)

\section{Results and Discussions}

Disorders in the silica lattice structure can induce defect points with localized energy states. These states, depending on their energy relative to the band gap, contribute to the density of electron or hole traps in the silica lattice. This can be clearly seen in the TDOS curve of the unmodified silica in Figure 6.2. A rather large density of hole traps is observed at the tail of the valence band, inside the band gap. The TDOS also extends over $1.5 \mathrm{eV}$ below the LUMO level. This indicates that the mere introduction of silica NPs to the dielectric material may introduce trapping states which can hinder the electrons and holes to move freely to the conduction and valence bands, respectively. The valence and conduction band offsets ( $\Delta E_{v}$ and $\Delta E_{c}$, respectively) between silica and APTES are rather large $(\sim 3.3 \mathrm{eV})$, and have the same sign. Grafting these two structures would create a situation where electrons accumulate on the lower energy side (silica), and holes on the other one (APTES) ${ }^{29}$. Considering the LUMO and HOMO levels as the shallowest electron and hole traps, respectively, visualizing these MOs can indicate the distribution of electrons and holes in this structure (Figure 6.3). It is clear that the unoccupied MOs are located in the silica lattice, whereas the occupied MOs are observed near the silane modifying agent. In the presence of an external electric field, the induced potential would drive the electrons and holes to recombine, and create trapping induced conduction. The APTES molecule with 
a large density of valence states close to the band gap can introduce new electronic states (hole traps) with energies within the band gap of the silica. The presence of these states around the APTES modifying agent can be seen in Figures $6.3 \mathrm{c}$ and $6.3 \mathrm{~d}$. Therefore, grafting APTES to the silica surface can further decrease the mobility of charges and contribute to the enhancement of the insulating properties of the system. The TDOS of APTES modified silica clearly indicates the existence of such states at the top range of the valence band, with the highest density of valence states residing $3.8 \mathrm{eV}$ below the band gap. In addition, the electron traps at the tail of the conduction band in the APTES modified silica are slightly $(0.6 \mathrm{eV})$ deeper than those of the untreated silica. This suggests that the electron traps are slightly shifted towards lower energies (deeper traps), when APTES is present on the surface. Since the band gap of the modified silica is significantly narrower than that of the untreated silica, the presence of these deep traps is crucial to maintain low carrier mobility under high electric fields.

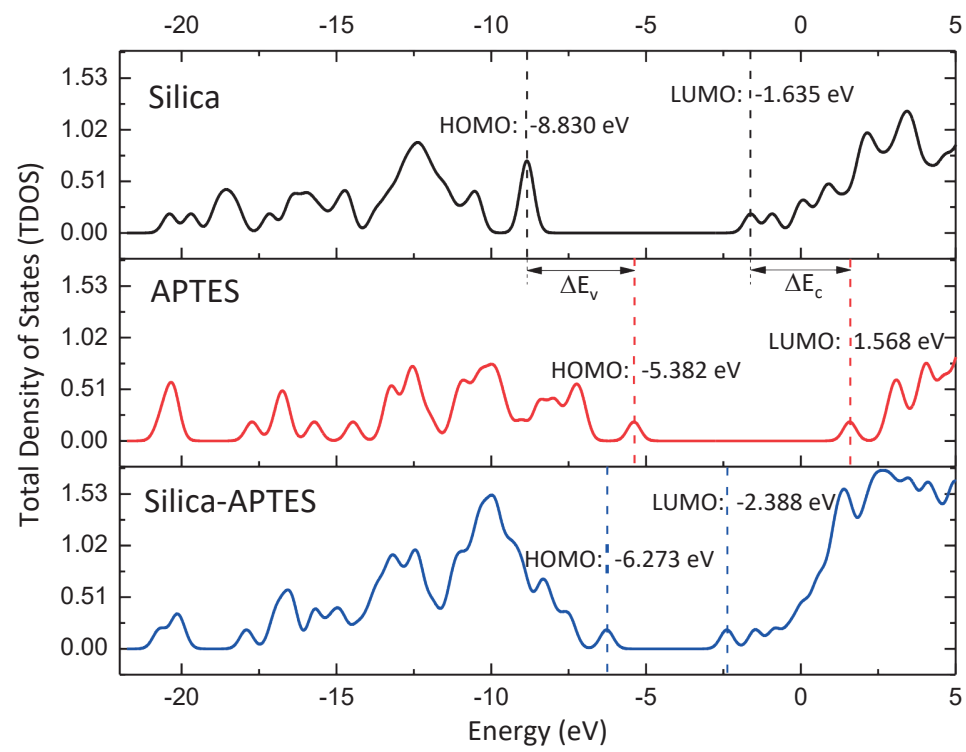

Figure 6.2. TDOS of the three studied model materials. $\Delta E_{v}$ and $\Delta E_{c}$ are band offsets of the two components: the difference between the valence and conduction band extrema in the band structures of silica and APTES. The lines matching each curve's color represent the HOMO and LUMO levels for their respective models. 
a.

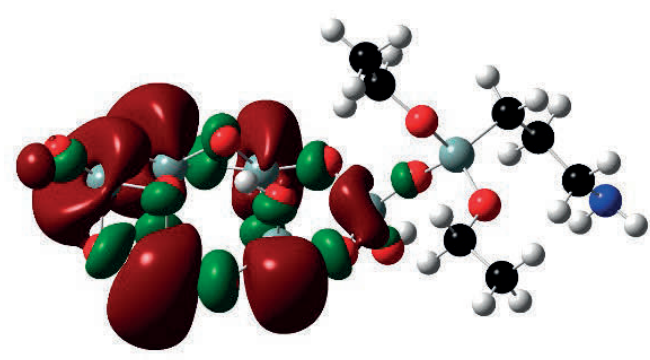

C.

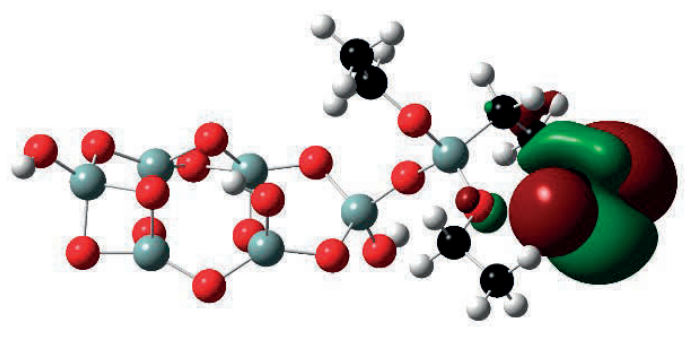

b.

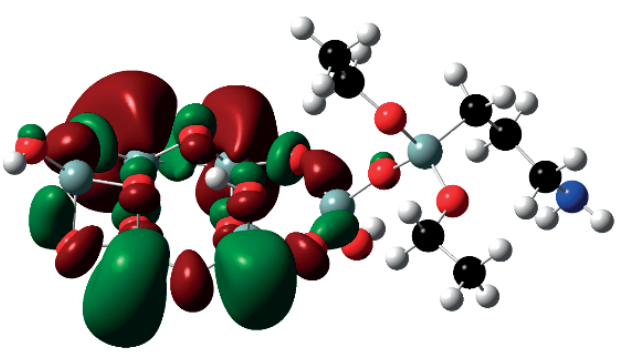

d.

Figure 6.3. a: Lowest unoccupied molecular orbitals (LUMO) ; b: LUMO+1; c: highest occupied molecular orbitals (HOMO) and d: $\mathrm{HOMO}+1$ visualized for the APTES grafted silica; (gray: silicon, light-red: oxygen, black: carbon, blue: nitrogen and white: hydrogen; the dark-red and green color of the MOs are related to the positive and negative wavefunctions, respectively)

So far, based on the DFT results, we have established that the presence of APTES on the silica surface can induce new electronic states beneficial for the insulating properties of their corresponding NCs. To demonstrate this experimentally, a process similar to atomic layer deposition (ALD) is utilized to deposit layers of APTES on the silica NPs in a controlled fashion by sequential dosing of gaseous APTES and water into a fluidized bed. Figure 6.4 
presents the aminopropylsiloxane (APS) grafting density on silica NPs at different numbers of APTES $/ \mathrm{H}_{2} \mathrm{O}$ cycles.

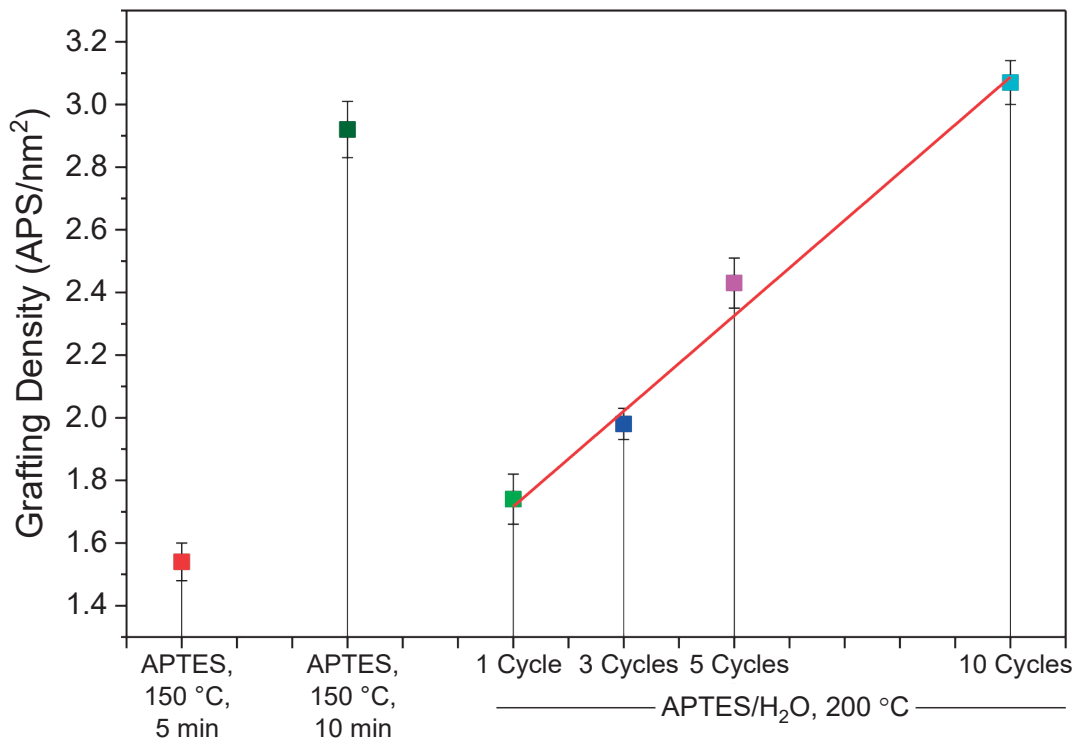

Figure 6.4. APS grafting density on silica NPs for different silica samples. The linear growth of the deposited film resembles the typical characteristic of an ALD process.

The linear growth of the homogenous APTES film can be clearly observed. After 10 APTES/ $\mathrm{H}_{2} \mathrm{O}$ cycles, the NPs surface is covered with a density of over $3 \mathrm{APS} / \mathrm{nm}^{2}$. The lower reaction temperature in these cases results in oligomerization of APTES, likely due to the presence of water on the NPs surface upon ambient exposure prior to the deposition. Therefore, APTES at $150{ }^{\circ} \mathrm{C}$ does not deposit in a self-limiting manner, likely leading to less control over the thickness and homogeneity, as opposed to the APTES $/ \mathrm{H}_{2} \mathrm{O}$ films which exhibit a saturating behavior, thus tending to be more homogeneously deposited over the reactive surface area of the NPs. The APTES depositions at $150^{\circ} \mathrm{C}$ for 5 and 10 min lead to comparable grafting densities to APTES/ $\mathrm{H}_{2} \mathrm{O}$ depositions at $200{ }^{\circ} \mathrm{C}$ after 1 and 10 cycles, respectively. This is also evident by the intensities of $\mathrm{C}-\mathrm{H}$ and $\mathrm{N}-\mathrm{H}$ bands in Figure 6.5a which are the highest in case of the samples with higher grafting densities. Despite the similar grafting densities, the deposition tends to be different when the APTES/ $\mathrm{H}_{2} \mathrm{O}$ cycles are applied. This is manifested in the C-O stretching band at $1150-1250 \mathrm{~cm}^{-1}$. In the case of deposition at $150{ }^{\circ} \mathrm{C}$, the higher intensity of the $\mathrm{C}-\mathrm{O}$ band suggests that non-hydrolyzed 
ethoxy groups from APTES are present in the deposited film. This is evidence of the higher number of dangling un-reacted ethoxy groups on the NP surface in this case compared to the APTES $/ \mathrm{H}_{2} \mathrm{O}$ depositions. In case of the deposition at $150{ }^{\circ} \mathrm{C}$, increasing the reaction time has resulted in further consumption of the phisysorbed and chemisorbed water on the silica surface after which more un-reacted ethoxy groups remain in the film. This is likely the reason for the higher intensity of the $\mathrm{C}-\mathrm{O}$ band for the film deposited in $10 \mathrm{~min}$. These observations indicate that the self-limiting deposition results in a more complete hydrolysis of the precursor, and reduces the number of dangling ethoxygroups. Furthermore, the growth of the film upon consecutive APTES/ $\mathrm{H}_{2} \mathrm{O}$ cycles is clearly observed in Figure $6.5 \mathrm{~b}$ as the gradual increase in the intensities of $\mathrm{C}-\mathrm{H}$ and $\mathrm{N}-\mathrm{H}$ bands.

\section{a.}

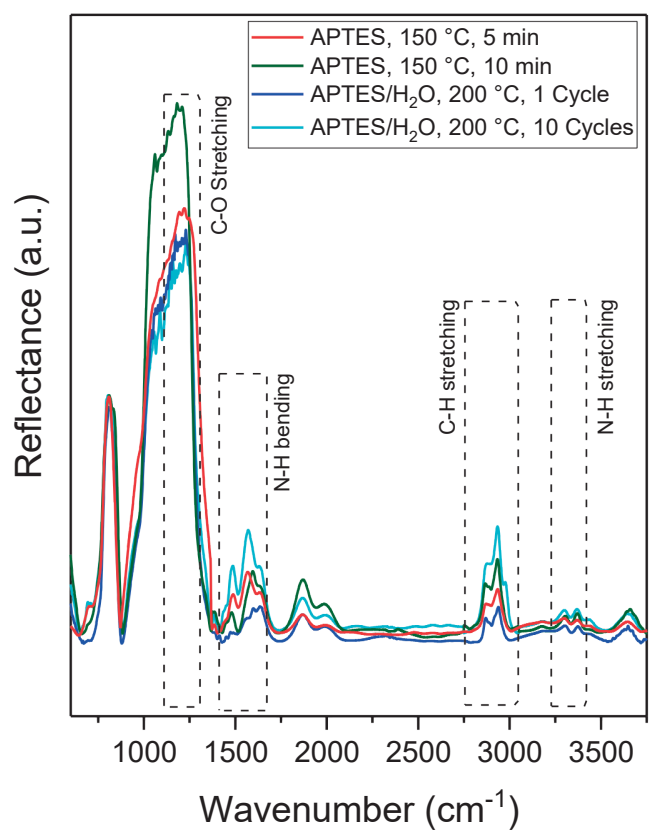

b.

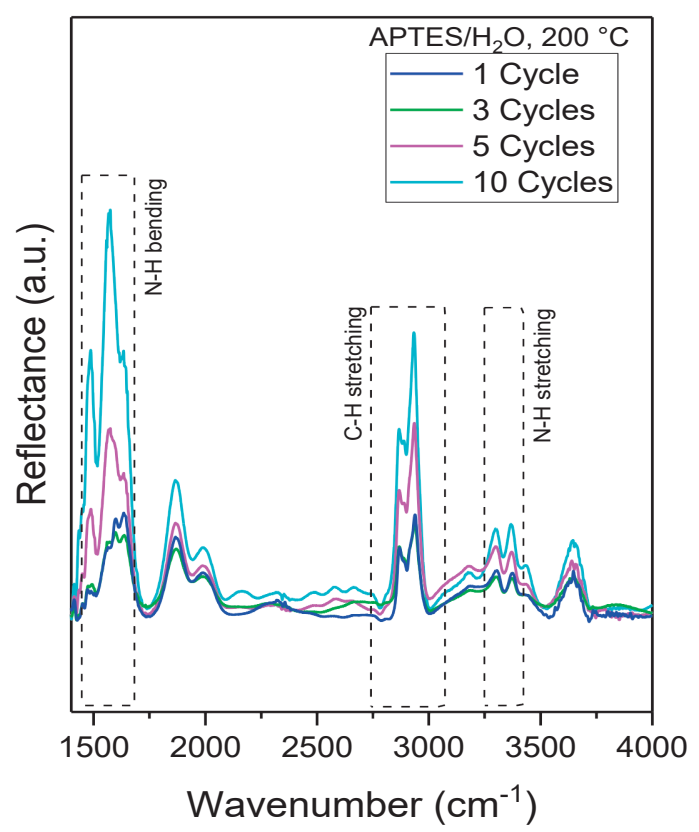

Figure 6.5. a: DRIFTS spectra of the NPs modified with and without the ALD-like cyclic approach, along with $b$ : the spectra of the APTES/ $\mathrm{H}_{2} \mathrm{O}$ modified NPs in different number of cycles. The DRIFTS spectrum of the untreated silica was presented in Chapter 5 
Since the surface properties of the NPs directly affect their nucleating ability in the polymer matrix, differential scanning calorimetry (DSC) was performed to study the effect of such surface functionalization on the crystallization behavior of our studied NCs. While no significant difference is observed in the melting curves (Figure 6.6a), the crystallization behavior undergoes meaningful changes upon incorporation of silica and its functionalization (Figure 6.6b). Despite the expected nucleating effect of the NPs ${ }^{30}$, the onset of crystallization slightly shifts to a lower temperature after addition of the untreated silica (Sample 0). It has been shown that the addition of a small concentration of $\mathrm{CaCO}_{3} \mathrm{NPs}$ to PP does in fact decrease the onset of crystallization ${ }^{31}$. So, there may be a minimum amount of NPs needed to effectively nucleate PP crystals, which may have not been achieved in our case with $1 \%$ of untreated silica. Nevertheless, the onset of crystallization increases by 5 to $10{ }^{\circ} \mathrm{C}$ upon functionalization of the NPs (see Table 6.1), indicating that despite the low concentration of the filler, the deposited APTES facilitates nucleation. The NPs modified at $150{ }^{\circ} \mathrm{C}$ have resulted in a higher onset of crystallization compared to the APTES/ $\mathrm{H}_{2} \mathrm{O}$ modified ones. This is likely due to the irregularities from the non-hydrolyzed ethoxy groups which resulted in an enhanced nucleation in these NCs. It is noteworthy that these changes are only observed in the crystallization peak of the PP phase, suggesting that the NPs are residing in the PP domains rather than in the EOC. All the APTES/ $\mathrm{H}_{2} \mathrm{O}$ modified NCs exhibit very similar crystallization curves suggesting that the self-limiting deposition of aminopropylsiloxane networks on the NPs has resulted in similar nucleation processes in these NCs. 
a.

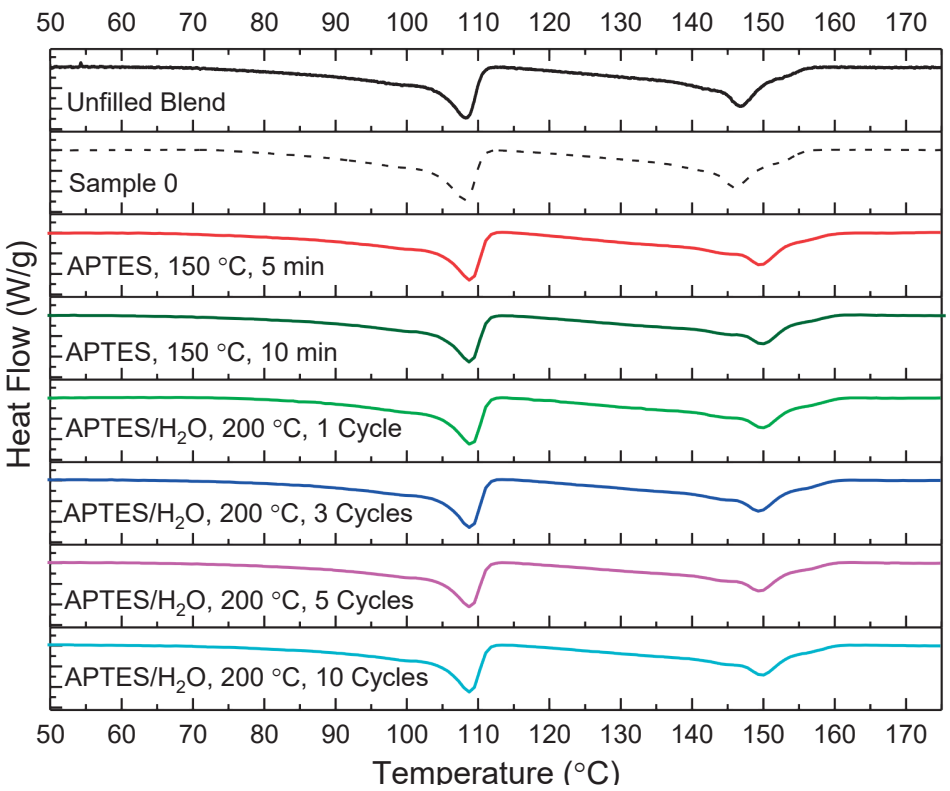

b.

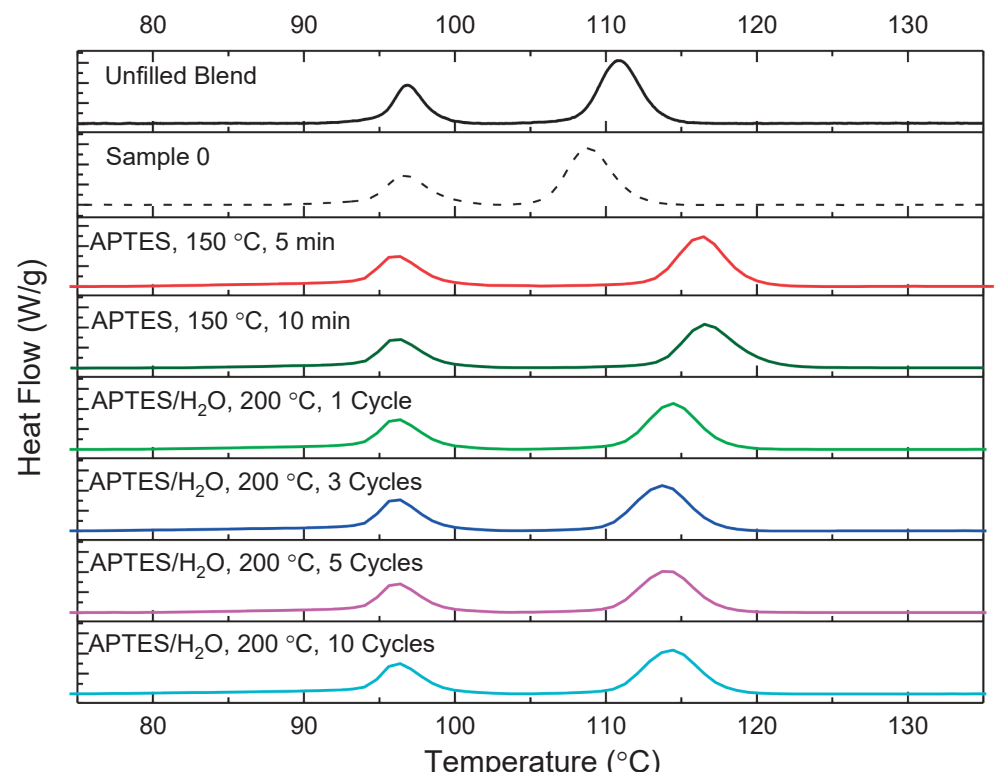

Figure 6.6. a: Melting curves ( $2^{\text {nd }}$ melting) of all the studied samples. Two melting peaks are observed around $109{ }^{\circ} \mathrm{C}$ and $150{ }^{\circ} \mathrm{C}$, which correspond to melting of the EOC and PP domains, respectively. b: Crystallization curves of the studied systems exhibiting two peaks. The EOC crystallization peak occurs at around $97^{\circ} \mathrm{C}$, and remains unchanged upon addition of the NPs and their surface functionalization. 
Table 6.1. DSC characteristic parameters

\begin{tabular}{lccc} 
& $\begin{array}{c}\text { Onset of } \\
\text { Crystallization }\left({ }^{\circ} \mathrm{C}\right)\end{array}$ & $\begin{array}{c}\text { Enthalpy of Melting } \\
(\mathbf{J} / \mathbf{g})\end{array}$ & $\begin{array}{c}\text { Percentage of } \\
\text { Crystallinity (\%) }\end{array}$ \\
\hline Unfilled Blend & 114 & 77.3 & 31 \\
Sample 0 & 112 & 74.9 & 30 \\
APTES, $150{ }^{\circ} \mathrm{C}, 5 \mathrm{~min}$ & 120 & 73.2 & 29 \\
APTES, $150{ }^{\circ} \mathrm{C}, 10 \mathrm{~min}$ & 122 & 78.1 & 32 \\
APTES $/ \mathrm{H}_{2} \mathrm{O}, 200^{\circ} \mathrm{C}, 1 \mathrm{Cycle}$ & 118 & 68.5 & 29 \\
APTES $/ \mathrm{H}_{2} \mathrm{O}, 200^{\circ} \mathrm{C}, 3$ Cycles & 117 & 70.1 & 30 \\
APTES $/ \mathrm{H}_{2} \mathrm{O}, 200^{\circ} \mathrm{C}, 5$ Cycles & 118 & 68.8 & 29 \\
APTES $/ \mathrm{H}_{2} \mathrm{O}, 200^{\circ} \mathrm{C}, 10$ Cycles & 117 & 69.2 & 29
\end{tabular}

In order to compare the dispersion of NPs in the polymer matrix, SEM was performed on the NCs. It is clear in Figure 6.7 that the PP/EOC blend exhibits a two-phase morphology, with the EOC domains elongated in the direction of the flow in the mold while passing through the solidified envelope at the walls of the mold ${ }^{6}$. It was also reported that the treatment of silica NPs with APTES results in the reduction of the mean NP aggregate size when treated in liquid phase. The same behavior is observed for the gas phase modified silica. A mean aggregate size of $160 \mathrm{~nm}$ is achieved with high grafting densities of APTES on silica (APTES_10 and 10 Cycles), whereas larger agglomerates were observed for low grafting density samples (APTES_5 and 1 Cycle). There is no significant difference between the dispersion of the APTES-modified silica at $150{ }^{\circ} \mathrm{C}$ and the APTES $/ \mathrm{H}_{2} \mathrm{O}$ modified one at $200{ }^{\circ} \mathrm{C}$ with the same grafting density. Moreover, as suggested by the DSC results, it is evident that the silica agglomerates are mostly located in the PP phase rather than EOC. This is likely due to the difference in the melt viscosities of the two phases, leading the silica to reside in the PP phase with lower melt viscosity ${ }^{32} 33$. 

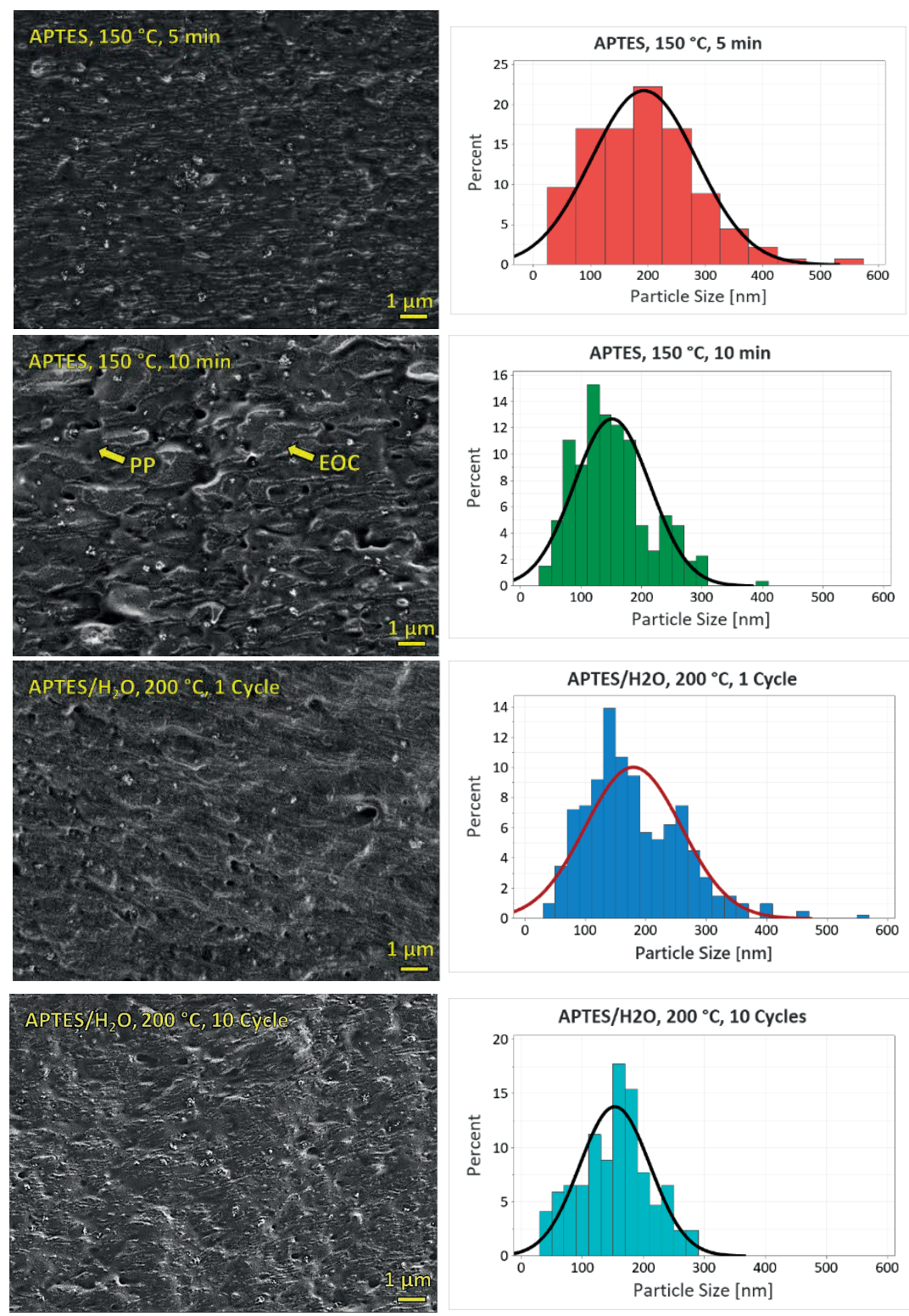

Figure 6.7. SEM images of NCs containing silica samples with self-limiting ( 1 and 10 cycles) and nonself-limiting modifications, along with the particle size histograms obtained from image processing software ImageJ with trainable Weka segmentation plugin ${ }^{34}$. The two polymer domains can be distinguished in the images. 
The TSDC spectra of NCs filled with these silica samples, in comparison with the spectra of the unfilled blend and the NC with untreated silica, are depicted in Figure 6.8a. The trap level and density distribution are numerically calculated from the TSDC data, using a method reported by Tian et al. ${ }^{35}$, and presented in Figure $6.8 \mathrm{~b}$. The TSDC spectrum of the unfilled polymer blend exhibits a relaxation peak at $74{ }^{\circ} \mathrm{C}$, which indicates the existence of traps with a distribution of depths around $0.98 \mathrm{eV}$ (see Figure 6.8b). These are likely charges released from the interfacial regions between the two polymer domains, or the crystalline/amorphous interfaces within either polymer phase. Upon incorporation of the silica (Sample 0), the peak trap density appears at a slightly deeper level compared to the unfilled blend. Evidently, introducing the amino-functionalized NPs tends to create even deeper states compared to the reference Sample 0, which is also well in-line with the DFT prediction. In addition, increasing the number of APTES/ $\mathrm{H}_{2} \mathrm{O}$ cycles results not only in a shift of the trap energies to higher (deeper) levels, but also a suppression of the occupied trap densities. The shifting of the traps to deeper levels upon NP modification in 10 cycles has also resulted in a reduced DC conductivity $\left(5.83 \times 10^{-16} \mathrm{~S} / \mathrm{m}\right)$, compared to the Sample 0 $\left(3.38 \times 10^{-15} \mathrm{~S} / \mathrm{m}\right)$ and the unfilled blend $\left(1.5 \times 10^{-15} \mathrm{~S} / \mathrm{m}\right)$. 

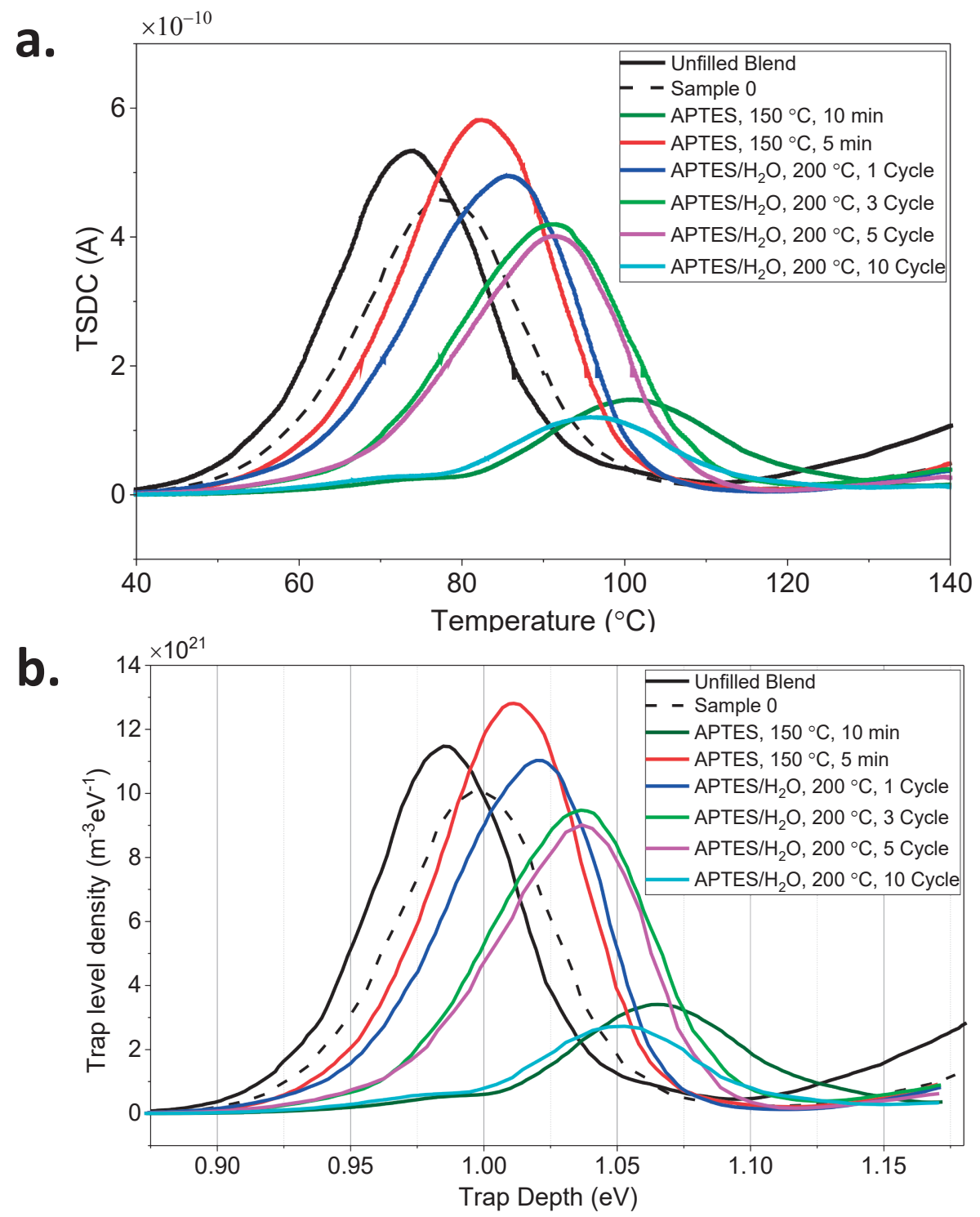

Figure 6.8. a: TSDC spectra and b: trap level distribution and density for all NCs and the unfilled reference. 

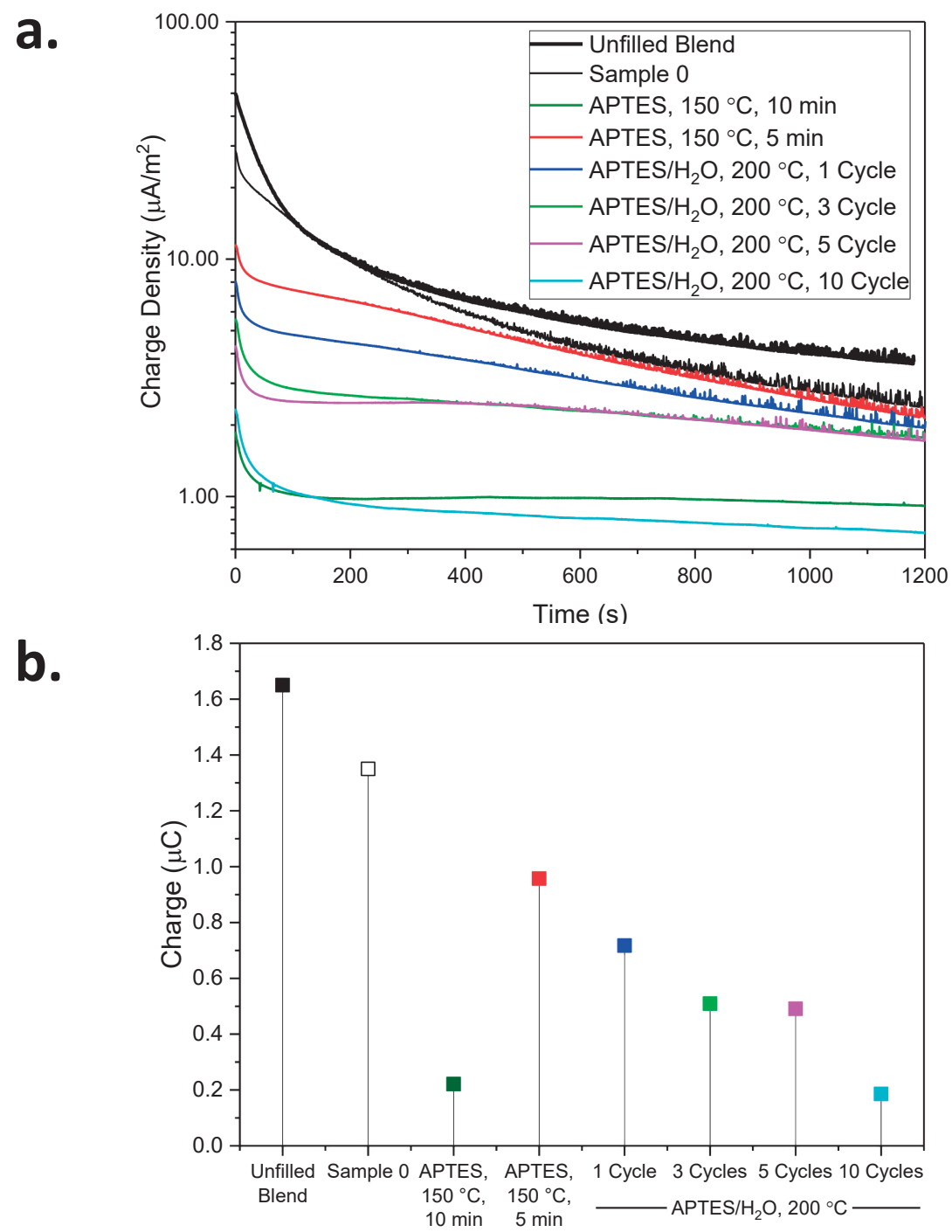

Figure 6.9. a: current density and b: amount of injected charge during the poling step for all NCs and the unfilled reference.

As the water pulse at the end of each APTES/ $\mathrm{H}_{2} \mathrm{O}$ cycle hydrolyzes the remaining ethoxy groups, adjacent $\mathrm{Si}-\mathrm{OH}$ moieties in the film can react and form siloxane bridges. The non-self-limiting deposition at $150^{\circ} \mathrm{C}$ on NPs may result in defect points with a broad range of energy levels at the filler-polymer interface. Comparing APTES depositions at $150{ }^{\circ} \mathrm{C}$ with APTES/ $\mathrm{H}_{2} \mathrm{O}$ ones at $200{ }^{\circ} \mathrm{C}$ with similar grafting densities, it is evident that the self-limiting deposition of APTES has resulted in a lower apparent density of the occupied traps at the 
filler-polymer interfaces. Moreover, deposition of APTES onto the silica NPs has resulted in lower current densities during the poling step of the TSDC test (Figure 6.9a). In the case of higher grafting densities of APTES, a quasi-steady state is achieved after about $150 \mathrm{~s}$, whereas the reference samples exhibit a decreasing current density throughout the whole polarization step. This is indicative of a rapid generation and recombination of charge at the beginning of the poling when the amine functional group is present at the filler-polymer interface. This is likely due to the created potential at the silica-APTES heterojunction, as discussed earlier. For the same reason, lower space charge is expected to accumulate at the interfaces in the modified samples. Figure $6.9 \mathrm{~b}$ represents the amount of injected charge during the poling step for all the samples. The decrease of injected charge upon increasing the number of APTES/ $\mathrm{H}_{2} \mathrm{O}$ cycles is clearly seen. Since the self-limiting deposition has resulted in a lower density of occupied traps, a relatively lower injection of charge is observed compared to the non-self-limiting cases with similar grafting densities. This may also be related to the presence of even deeper traps in the system which capture the charge and prevent further injection of the charges. It is worthwhile to mention that even though the presence of deep trapping states and lower space charge accumulation seems counterintuitive, a low density of deep traps, when occupied, can evidently hinder injection of electrons to the sample and reduce the formation of space charge.

Dielectric spectroscopy is a powerful tool to obtain information about material responses to an alternating electric field. The variations in real and imaginary parts of permittivity with respect to the frequency of the applied field can then be attributed to different polarization processes within the material. Considering Figures 6.10a and 6.10b, it is clear that at frequencies higher than $1 \mathrm{~Hz}$, little difference is observed in the real and imaginary permittivity of the unfilled blend and the NCs. In the low frequency range, however, the unfilled blend and Sample 0 exhibit a steep rise in both parts of permittivity. In general, this is attributable to either the relaxation of big dipolar species with relatively long relaxation times, or the presence of Maxwell-Wagner (space charge) relaxations ${ }^{36}$. The former is not likely to occur for we are dealing with a non-polar polymer matrix. Since the BDS data were taken after the TSDC, these low frequency variations can be related to the relaxation of space charge in the material accumulated during the TSDC test. It is clear that 
upon introduction of the silica, both bare and functionalized, this low frequency rise is suppressed to the point where only a marginal increase is observed for the 10 cycles sample. This behavior was also observed in the TSDC space charge relaxation peak (Figure 6.8a): charge injection was mitigated due to the presence of deep traps, thus the space charge related permittivity increase at low frequencies is not observed. Comparing different modified samples in Figure 6.10c, it is evident that increasing the number of APTES/ $\mathrm{H}_{2} \mathrm{O}$ cycles has resulted, in general, in higher values of real permittivity. APTES depositions at $150{ }^{\circ} \mathrm{C}$ exhibit lower real permittivities compared to the APTES/ $\mathrm{H}_{2} \mathrm{O}$ depositions at $200{ }^{\circ} \mathrm{C}$, despite similar grafting densities. Real permittivity is directly related to the number of polarizable species in the system. In case of depositions at $150{ }^{\circ} \mathrm{C}$, APTES may have formed larger oligomers due to the uncontrolled release of water, resulting in an overall lower number of polarizable species in the material. The APTES deposition at $150{ }^{\circ} \mathrm{C}$ also exhibits a wider range of APTES chain lengths compared to the APTES $/ \mathrm{H}_{2} \mathrm{O}$ depositions at $200{ }^{\circ} \mathrm{C}$ as observed in the Cole-Cole plot $\left(\varepsilon^{\prime \prime}\right.$ vs $\left.\varepsilon^{\prime}\right)$ presented in Figure 6.10e. For a dielectric with a single relaxation time, the Cole-Cole plot would exhibit a semi-circle, indicating a Debye-like relaxation ${ }^{37}$. Generally, polymer blends do not follow Debye's relaxation function, except at very high frequencies where the relaxation rate can be considered constant ${ }^{38}$. Expectedly, a deviation from such behavior is observed in the Cole-Cole spectra of our studied NCs at low frequencies. The extent of this deviation, however, is not the same for all the cases. With 10 APTES $/ \mathrm{H}_{2} \mathrm{O}$ cycles at $200{ }^{\circ} \mathrm{C}$, this deviation tends to be less pronounced compared to the deposition at $150^{\circ} \mathrm{C}$ for $10 \mathrm{~min}$ with a similar grafting density. This suggests that a narrower range of APTES chain lengths are grafted onto the silica in case of APTES/ $\mathrm{H}_{2} \mathrm{O}$ deposition. Another reason for this low frequency variation, as mentioned before, is the presence of space charge relaxations which is also observed in the imaginary permittivity in Figure $6.10 \mathrm{~d}$ at frequencies lower than $1 \mathrm{~Hz}$. The self-limiting deposition tends to decrease the space charge polarizations at the filler-polymer interfaces. This is more clearly seen comparing the APTES deposition at $150{ }^{\circ} \mathrm{C}$ for $10 \mathrm{~min}$ and the $10 \mathrm{cycle}$ APTES $/ \mathrm{H}_{2} \mathrm{O}$ one at $200{ }^{\circ} \mathrm{C}$. Also, the higher the number of APTES $/ \mathrm{H}_{2} \mathrm{O}$ cycles, the lower the low frequency rise of the imaginary permittivity, and therefore, lower susceptibility for space charge formation. 
a.

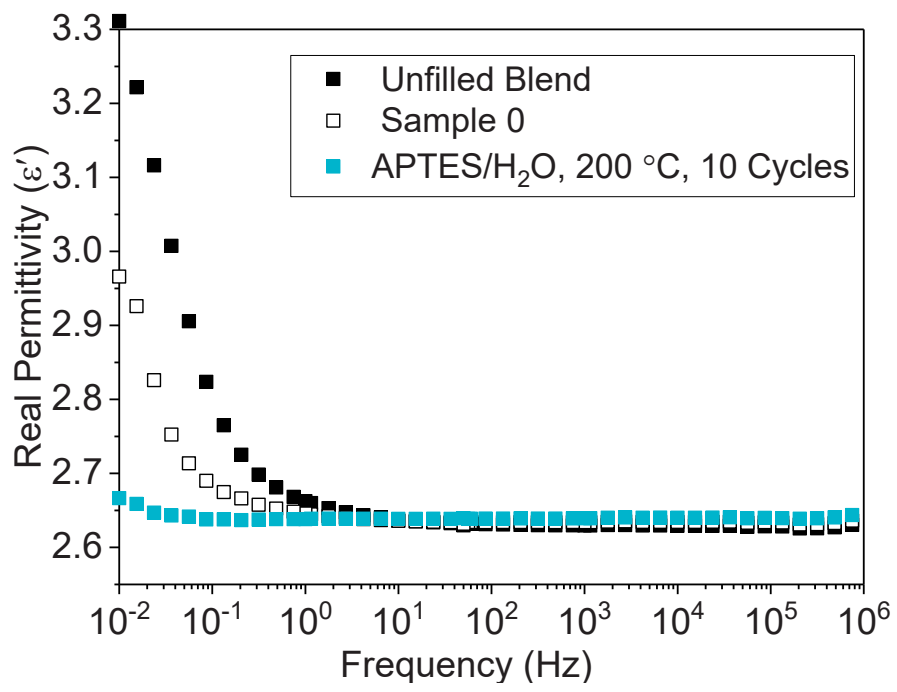

b.

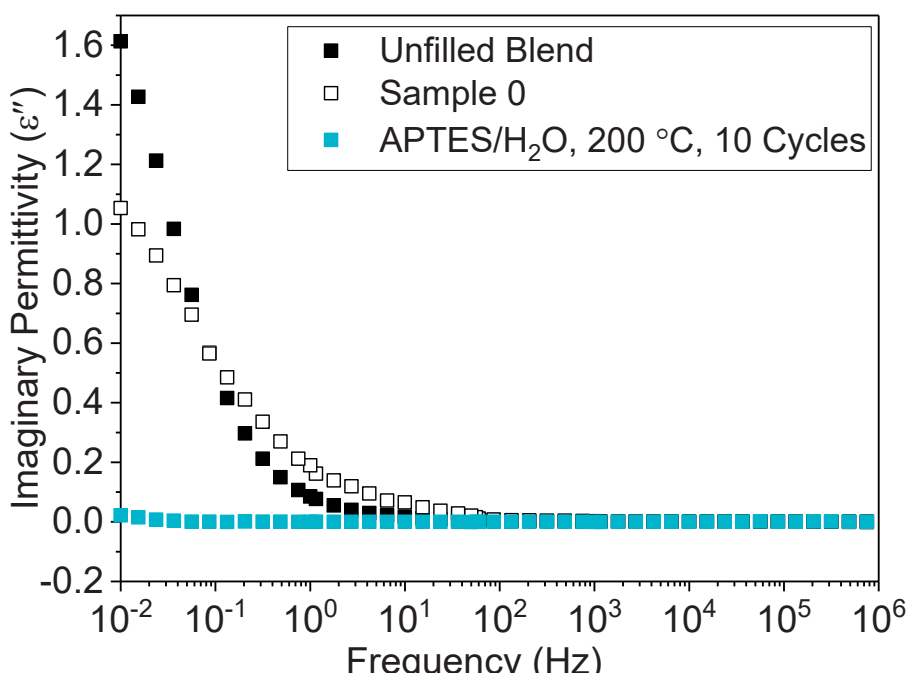


c.

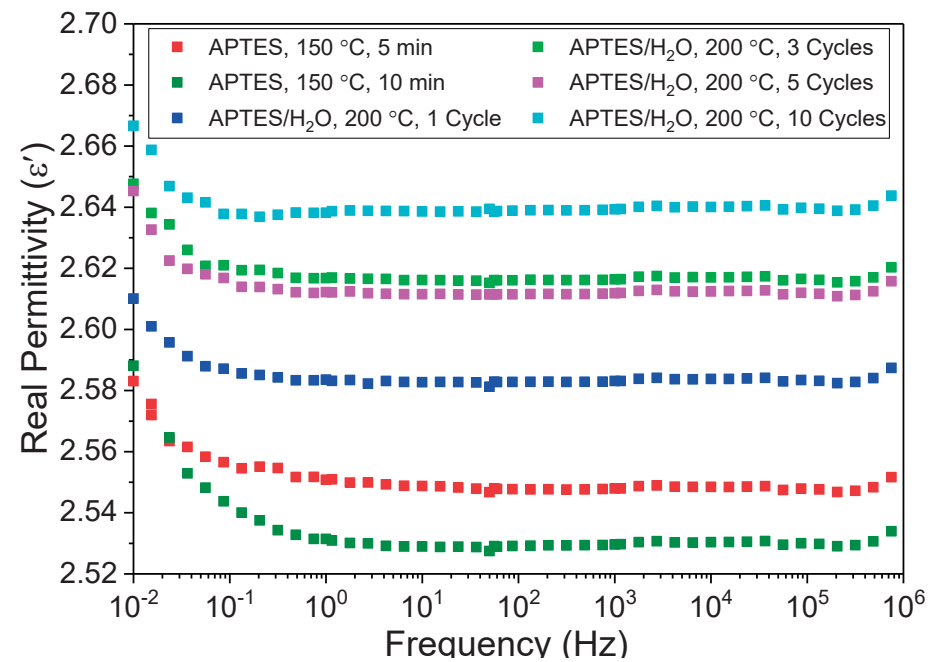

d.

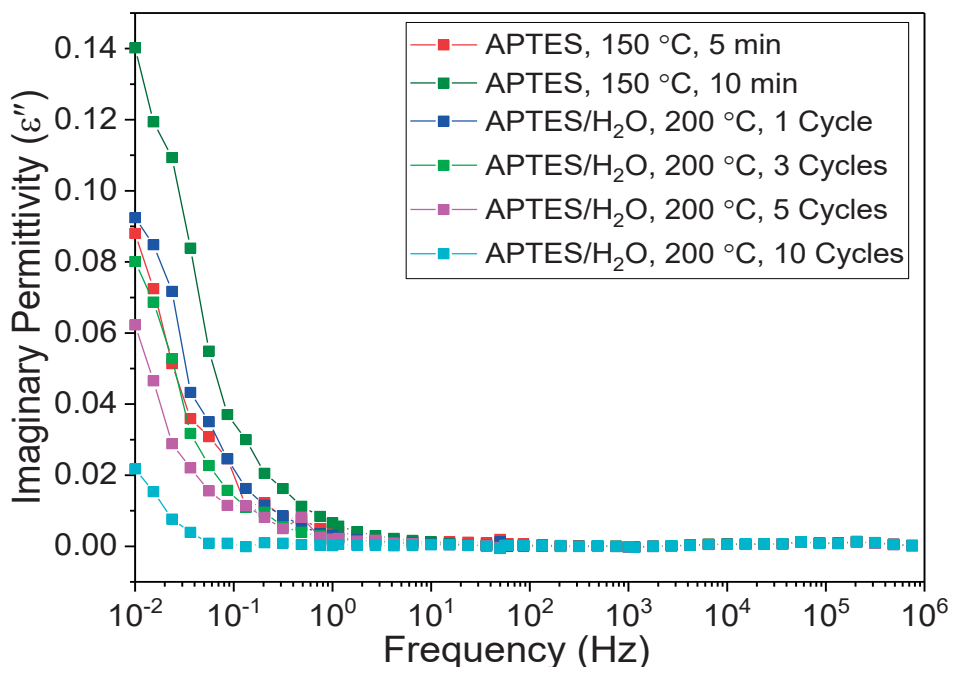




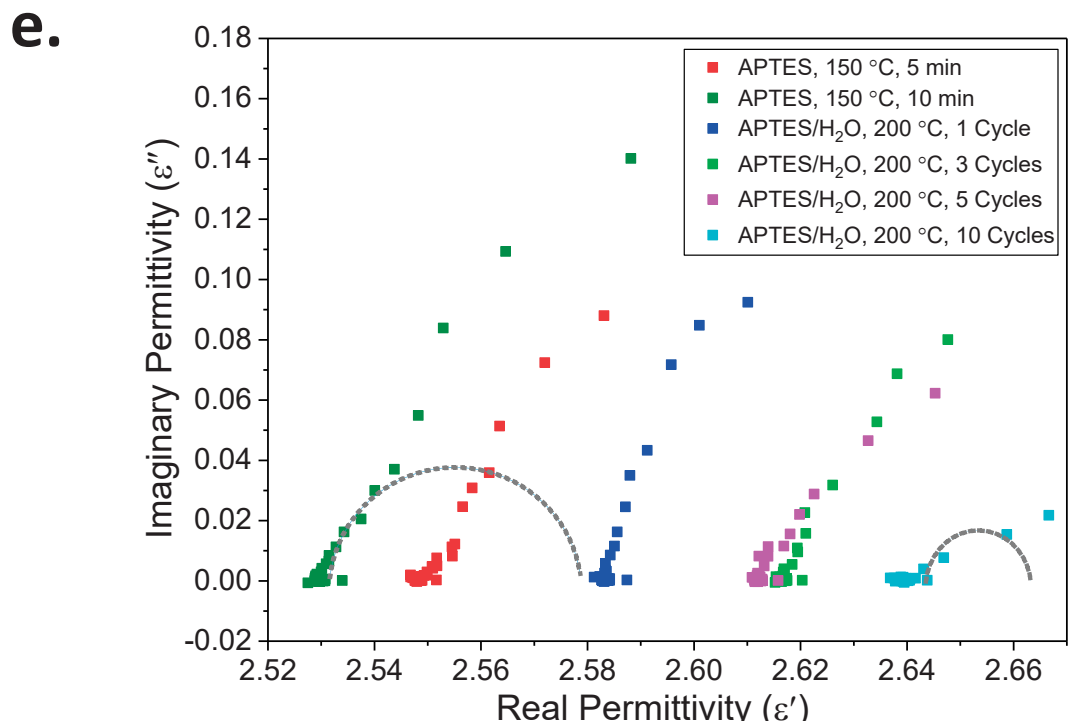

Figure 6.10. a, b: Real and imaginary parts of permittivity for the unfilled blend compared to NC's with modified and non-modified silica. c, d: Real and imaginary parts of permittivity for the different samples with modified silica. e: Cole-Cole plot of different modified samples

Complementing the DFT results, BDS and TSDC provide further evidence that the amino functionalized silica NPs induce deep trapping states that when occupied can suppress the further injection of charge and formation of space charge in the presence of the electric field. According to the Lewis's formulation of the interfacial interactions in nanodielectrics, space charge and conductivity are largely affected by the electric double layer that is superimposed near the filler-polymer interfaces ${ }^{39}$. The electric double layer contains mobile charge carriers that are partly under the influence of the NPs' surface charge. Therefore, the improvements observed above can be attributed, partly to the alterations of the surface charge and electronic states of the NPs, and partly to the morphological differences between the NCs.

The deposition process evaluated in this study offers outstanding control over the characteristics of the film, and is able to tailor the interfacial properties in insulating dielectric NCs. Furthermore, the scalability of this process allows for industrial scale production of NCs with superior dielectric performance ${ }^{40}$. 


\section{Conclusions}

Using density functional theory (DFT) calculations, we demonstrated the alterations in the electronic structure of the silica when modified with an electrically active amino functional group. The silica/APTES heterojunction exhibited a large density of valence states and a unique electron/hole distribution; when subjected to an external electric field, it can suppress the formation of space charge in the NCs. To confirm the DFT results experimentally, APTES was grafted onto silica NPs via a process analogous to atomic layer deposition (ALD) in a controllable layer-by-layer fashion. Thermally stimulated depolarization current (TSDC) and broadband dielectric spectroscopy (BDS) measurements were utilized to investigate the charge trapping and transport phenomena in the studied NCs. The DFT predictions were in good accordance with the experimental results. A low density of deep trapping states was introduced upon modification of the NPs with APTES which resulted in a lower current density and DC conductivity. Moreover, APTES/ $\mathrm{H}_{2} \mathrm{O}$ cyclic deposition at $200{ }^{\circ} \mathrm{C}$, which resulted in a self-limiting growth, delivered a narrower distribution of relaxation times and a reduced density of carrier traps on the silica surface. Due to the reduced density of traps and the lower space charge relaxations, these NC systems can be beneficial for designing long-lasting high voltage insulation systems.

\section{References}

[1] Fujita, S.; Ruike, M.; Baba, M. Treeing breakdown voltage and TSC of alumina filled epoxy resin, Proceedings of Conference on Electrical Insulation and Dielectric PhenomenaCEIDP'96, IEEE: 1996; pp 738-741.

[2] Khalil, M. S.; Henk, P. O.; Henriksen, M. The influence of titanium dioxide additive on the short-term DC breakdown strength of polyethylene, IEEE International Symposium on Electrical Insulation. Conference Record, Institute of Electrical and Electronics Engineers: 1990; pp 268-271.

[3] Ma, D.; Treese, A. H.; Siegel, R.; Anna, C.; Eva, M.; Carina, Ö.; Linda, S. S., Influence of nanoparticle surface modification on the electrical behaviour of polyethylene nanocomposites. Nanotechnology, 16 (6), 2005, p 724.

[4] Jiang, M.-J.; Dang, Z.-M.; Xu, H.-P., Enhanced electrical conductivity in chemically modified carbon nanotube/methylvinyl silicone rubber nanocomposite. European Polymer Journal, 43 (12), 2007, pp 4924-4930.

[5] Mathabani, A.; Rytöluoto, I.; He, X.; Saarimäki, E.; Lahti, K.; Paajanen, M.; Anyszka, R.; Dierkes, W.; Blume, A. Solution modified fumed silica and its effect on charge trapping 
behavior of PP/POE/silica nanodielectrics, Proceedings of the Nordic Insulation Symposium, 2019; pp 129-133.

[6] Mahtabani, A.; Rytöluoto, I.; Anyszka, R.; He, X.; Saarimäki, E.; Lahti, K.; Paajanen, M.; Dierkes, W.; Blume, A., On the Silica Surface Modification and Its Effect on Charge Trapping and Transport in PP-Based Dielectric Nanocomposites. ACS Applied Polymer Materials, 2 (8), 2020, pp 3148-3160.

[7] Dongling, M.; Treese, A. H.; Richard, W. S.; Anna, C.; Eva, M.; Carina, Ö.; Linda, S. S., Influence of nanoparticle surface modification on the electrical behaviour of polyethylene nanocomposites. Nanotechnology, 16 (6), 2005, p 724.

[8] Suzuoki, Y.; Muto, H.; Cai, G.-X.; Mizutani, T.; leda, M., Effects of Polar Groups on Electrical Conduction in Polyethylene. Japanese journal of applied physics, 23 (Part 1, No. 1), 1984, pp 91-92.

[9] He, X.; Mahtabani, A.; Rytöluoto, I.; Saarimäki, E.; Lahti, K.; Paajanen, M.; Anyszka, R.; Dierkes, W.; Blume, A. Surface Modification of Fumed Silica by Dry Silanization for PPbased Dielectric Nanocomposites, 2019 2nd International Conference on Electrical Materials and Power Equipment (ICEMPE), IEEE: 2019; pp 254-259.

[10] Parr, R. G. Density Functional Theory of Atoms and Molecules, Horizons of Quantum Chemistry, Springer Netherlands: Dordrecht, 1980; pp 5-15.

[11] Shahmohammadi, M.; Pensa, E.; Bhatia, H.; Yang, B.; Jursich, G.; Takoudis, C. G., Enhancing the surface properties and functionalization of polymethyl methacrylate with atomic layer-deposited titanium(IV) oxide. Journal of Materials Science, 55 (36), 2020, pp 17151-17169.

[12] King, D. M.; Liang, X.; Weimer, A. W., Functionalization of fine particles using atomic and molecular layer deposition. Powder Technology, 221, 2012, pp 13-25.

[13] Nee, A. Y., Handbook of manufacturing engineering and technology Springer: 2015; [14] Oviroh, P. O.; Akbarzadeh, R.; Pan, D.; Coetzee, R. A. M.; Jen, T.-C., New development of atomic layer deposition: processes, methods and applications. Science and technology of advanced materials, 20 (1), 2019, pp 465-496.

[15] Van Bui, H.; Grillo, F.; Van Ommen, J., Atomic and molecular layer deposition: off the beaten track. Chemical Communications, 53 (1), 2017, pp 45-71.

[16] George, S. M., Atomic layer deposition: an overview. Chemical Reviews, 110 (1), 2010, pp 111-131.

[17] Powell, Q. H.; Fotou, G. P.; Kodas, T. T.; Anderson, B. M.; Guo, Y., Gas-phase coating of TiO 2 with SiO 2 in a continuous flow hot-wall aerosol reactor. Journal of Materials Research, 12 (2), 1997, pp 552-559.

[18] M. J. Frisch, G. W. T., H. B. Schlegel, G. E. Scuseria, M. A. Robb, J. R. Cheeseman, G. Scalmani, V. Barone, G. A. Petersson, H. Nakatsuji, X. Li, M. Caricato, A. Marenich, J. Bloino, B. G. Janesko, R. Gomperts, B. Mennucci, H. P. Hratchian, J. V. Ortiz, A. F. Izmaylov, J. L. Sonnenberg, D. Williams-Young, F. Ding, F. Lipparini, F. Egidi, J. Goings, B. Peng, A. Petrone, T. Henderson, D. Ranasinghe, V. G. Zakrzewski, J. Gao, N. Rega, G. Zheng, W. Liang, M. Hada, M. Ehara, K. Toyota, R. Fukuda, J. Hasegawa, M. Ishida, T. Nakajima, Y. Honda, O. Kitao, H. Nakai, T. Vreven, K. Throssell, J. A. Montgomery, Jr., J. E. Peralta, F. Ogliaro, M. Bearpark, J. J. Heyd, E. Brothers, K. N. Kudin, V. N. Staroverov, T. Keith, R. Kobayashi, J. Normand, K. Raghavachari, A. Rendell, J. C. Burant, S. S. Iyengar, J. Tomasi, M. Cossi, J. M. Millam, M. 
Klene, C. Adamo, R. Cammi, J. W. Ochterski, R. L. Martin, K. Morokuma, O. Farkas, J. B. Foresman, and D. J. Fox, Gaussian 09, Revision A.02. Gaussian, Inc., 2016.

[19] Wu, C.; Wang, J.; Chang, P.; Cheng, H.; Yu, Y.; Wu, Z.; Dong, D.; Zhao, F., Polyureas from diamines and carbon dioxide: synthesis, structures and properties. Physical Chemistry Chemical Physics, 14 (2), 2012, pp 464-468.

[20] Becke, A. D., A new mixing of Hartree-Fock and local density-functional theories. The Journal of chemical physics, 98 (2), 1993, pp 1372-1377.

[21] Pritchard, B. P.; Altarawy, D.; Didier, B.; Gibson, T. D.; Windus, T. L., New Basis Set Exchange: An Open, Up-to-Date Resource for the Molecular Sciences Community. Journal of Chemical Information and Modeling, 59 (11), 2019, pp 4814-4820.

[22] Lu, T.; Chen, F., Multiwfn: A multifunctional wavefunction analyzer. Journal of Computational Chemistry, 33 (5), 2012, pp 580-592.

[23] Khavryuchenko, V. D.; Khavryuchenko, O. V.; Lisnyak, V. V., Formation of pyrogenic silica: spectroscopic and quantum chemical insight. Critical Reviews in Solid State and Materials Sciences, 36 (2), 2011, pp 47-65.

[24] Liebau, F., Structural chemistry of silicates: structure, bonding, and classification Springer Science \& Business Media: 2012;

[25] Revesz, A.; Hughes, H., The structural aspects of non-crystalline SiO2 films on silicon: a review. Journal of Non-Crystalline Solids, 328 (1-3), 2003, pp 48-63.

[26] Khavryutchenko, V.; Barthel, H.; Nikitina, E. Fumed silica synthesis: from molecules, protoparticles and primary particles to aggregates and agglomerates, Macromolecular Symposia, Wiley Online Library: 2001; pp 7-18.

[27] Ulrich, G. D.; Rieh, J. W., Aggregation and growth of submicron oxide particles in flames. Journal of Colloid and Interface Science, 87 (1), 1982, pp 257-265.

[28] Blume, A.; Jin, J.; Mahtabani, A.; He, X.; Kim, S.; Andrzejewska, Z. J. New Structure Proposal for Silane Modified Silica, International Rubber Conference, IRC 2019, 2019.

[29] Vasko, F. T.; Kuznetsov, A. V., Electronic states and optical transitions in semiconductor heterostructures Springer Science \& Business Media: 2012;

[30] Abbasi-Shirsavar, M.; Baghani, M.; Taghavimehr, M.; Golzar, M.; Nikzad, M.; Ansari, M.; George, D., An experimental-numerical study on shape memory behavior of PU/PCL/ZnO ternary blend. Journal of Intelligent Material Systems and Structures, 30 (1), 2018, pp 116-126.

[31] Avella, M.; Cosco, S.; Di Lorenzo, M. L.; Di Pace, E.; Errico, M. E.; Gentile, G., Nucleation activity of nanosized $\mathrm{CaCO} 3$ on crystallization of isotactic polypropylene, in dependence on crystal modification, particle shape, and coating. European Polymer Journal, 42 (7), 2006, pp 1548-1557.

[32] He, X.; Seri, P.; Rytöluoto, I.; Anyszka, R.; Mahtabani, A.; Naderiallaf, H.; Niittymäki, M.; Saarimäki, E.; Mazel, C.; Perego, G.; Lahti, K.; Paajanen, M.; Dierkes, W.; Blume, A., Dielectric Performance of Silica-Filled Nanocomposites Based on Miscible (PP/PP-HI) and Immiscible (PP/EOC) Polymer Blends. IEEE Access, 9, 2021, pp 15847-15859.

[33] Spikes, H. A., A Thermodynamic Approach to Viscosity. Tribology Transactions, 33 (1), 1990, pp 140-148.

[34] Ignacio, A.-C.; Verena, K.; Curtis, R.; Kevin W, E.; Johannes, S.; Cardona, A.; Seung, H. S., Trainable Weka Segmentation: a machine learning tool for microscopy pixel classification. Bioinformatics, 33 (15), 2017, pp 2424-2426. 
[35] Tian, F.; Bu, W.; Shi, L.; Yang, C.; Wang, Y.; Lei, Q., Theory of modified thermally stimulated current and direct determination of trap level distribution. Journal of Electrostatics, 69 (1), 2011, pp 7-10.

[36] Nelson, J. K.; Fothergill, J. C., Internal charge behaviour of nanocomposites. Nanotechnology, 15 (5), 2004, p 586.

[37] Powles, J. G., Dielectric Relaxation and the Internal Field. The Journal of chemical physics, 21 (4), 1953, pp 633-637.

[38] Isayev, A. I., Encyclopedia of Polymer Blends, Volume 3: Structure John Wiley \& Sons: 2016;

[39] Lewis, T., Interfaces: nanometric dielectrics. Journal of Physics D: Applied Physics, 38 (2), 2005, p 202.

[40] Van Ommen, J.; Goulas, A., Atomic layer deposition on particulate materials. Materials Today Chemistry, 14, 2019, p 100183. 



\section{CHAPTER 7}

Fluidized Bed Molecular Layer

Deposition of Polyurea 


\section{Abstract}

Molecular layer deposition (MLD) is a robust method that is able to deposit organic layers onto substrates as well as nanoparticles in a layer by layer fashion. MLD utilizes selflimiting reactions of two or more precursors with an active surface, and chemically grafts the desired material by consecutive dosing of each precursor. Accordingly, the feasibility of this method is evaluated for modifying the surface of fumed silica nanoparticles in a fluidized bed reactor. Phenyldiisocyanate (PDIC) and ethylenediamine (ED) are used as precursors. After verification of the self-limiting feature, different number of MLD cycles are applied to grow polyurea films on silica in a controlled manner. This type of surface modification enables outstanding control over the chemical composition of the deposited layer, which can be beneficial for tailoring the interfacial properties in dielectric nanocomposites. 


\section{Introduction}

Functionalization of particle surfaces is commonly done via sol-gel techniques in liquid phase ${ }^{1-3}$. However, liquid phase processes result in inconsistent uncontrollable coatings. Moreover, they often tend to be relatively complex, resources and time-consuming due to the solvent/precursor waste and the need for separation processes after the reaction ${ }^{4}$. Therefore, vapor-phase processes are preferable for depositing conformal organic layers on solid surfaces in an efficient way. Chemical vapor deposition (CVD) is the most common vapor phase method for functionalizing surfaces, where reactive gaseous precursors are delivered to the reactor simultaneously ${ }^{5}$. However, CVD depositions tend to be nonconformal as this method offers no control over the thickness of the coating, yielding potential batch-to-batch variations. To overcome this issue, Yoshimura et al. introduced Molecular layer deposition (MLD) in the 1990s, as a robust film growth technique that is capable of depositing organic layers on substrates in a controlled manner ${ }^{6}$. Similarly to its inorganic counterpart, atomic layer deposition (ALD), MLD utilizes self-saturating reactions between bifunctional precursors to grow organic films on solid surfaces in a layer-by-layer fashion ${ }^{7}$. In particular, the target surfaces are coated via sequential pulses of the gaseous precursors, separated by a purging step with an inert gas, to minimize the uncontrolled CVD reactions in the reactor. Various studies have focused on depositing polymer MLD films on a variety of substrates ${ }^{8-14}$. However, the applicability of this approach has not been extensively studied on nanoparticles. Most recently, La Zara et al. reported MLD of ultrathin poly (ethylene terephthalate) (PET) films on $\mathrm{TiO}_{2}$ nanoparticles to suppress their photoactivity and enhance their dispersibility ${ }^{15}$. In another study, Gil-Font et al. demonstrated successful deposition of PET MLD films on tin nanoparticles, to improve their dispersibility in a thermal oil for an enhanced heat transfer ${ }^{16}$.

The controllability of MLD enables conformal reproducible depositions on the nanoparticles, which is of great importance in nanocomposite applications where the interface between the nanoparticle and the host polymer matrix determines the performance of the material ${ }^{17}$. It was shown in the previous chapter that a conformal coating of nanoparticles is preferred, as it reduces the irregularities and dangling bonds on 
the filler and hence the occupied trap density. Furthermore, chemical grafting of electrically active moieties as an effective approach to alter charge trapping properties in dielectric nanocomposites, is also possible through MLD. Accordingly, polyurea is a good candidate to be deposited on the nanoparticles, as its backbone contains amide and phenyl functional groups susceptible to alter the electronic structure of the filler surface in favor of the dielectric properties. While MLD was utilized to deposit polyurea films on flat silica substrates ${ }^{18-22}$, it has not yet been applied on nanoparticles in fluidized beds.

In this chapter, polyurea films are deposited on fumed silica nanoparticles using fluidized bed molecular layer deposition for the first time. The MLD process is carried out in a vibrating fluidized bed reactor to ensure efficient exposure of the reactive silanol groups on the nanoparticles (NPs) to the gaseous precursors. Phenyldiisocyanate (PDIC) and ethylenediamine (ED) are used as precursors (see Figure 7.1). Since PDIC can readily react with silanol groups on the silica ${ }^{20}$, no pre-treatment is necessary prior to the MLD process. The formation of urea segments can proceed by sequential dosing of the two precursors into the fluidized bed using nitrogen as the carrier gas. Thermogravimetric Analysis (TGA) is used to verify the self-limiting behavior of the process, and to quantify the amount of organic matter deposited on the NPs. Diffuse Reflectance Fourier Transform IR spectroscopy DRIFTS) and X-Ray Photoelectron Spectroscopy (XPS) are utilized to confirm the chemical composition of the deposited polyurea films. 


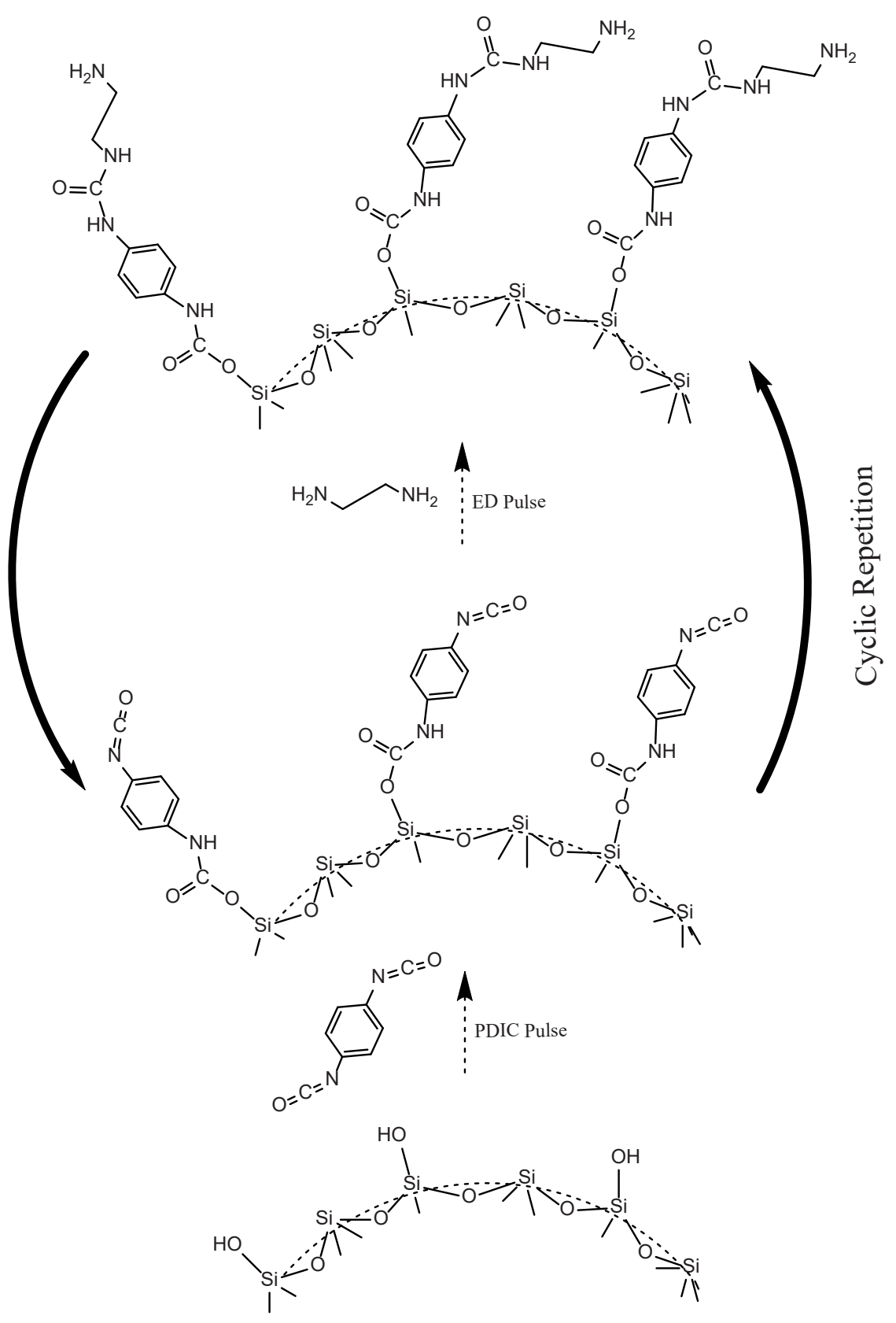

Figure 7.1. The chemical structures of PDIC and ED and the resulting urea units on the silica nanoparticles 


\section{Experimental Work}

\subsection{Materials}

AEROSIL 200 silica nanoparticles (primary particle size of $12 \mathrm{~nm}$, specific surface area of $\sim 200 \mathrm{~m}^{2} / \mathrm{g}$ ), with high purity and low moisture content, was received from Evonik Industries, Germany. The NPs were kept in an oven over night at $120^{\circ} \mathrm{C}$ to remove the physisorbed water. Phenyldiisocyanate (PDIC) and ethylenediamine (ED) (Sigma Aldrich, Germany) were used without any additional purification. Precursors were weighed and placed into stainless steel bubblers inside a glove box under inert environment. Mineral oil Kaydol (Sonneborn, USA) and sodium hydroxide (ABCR, Germany) were used in washing bubblers in the MLD setup. $\mathrm{KBr}(99+\%$, FTIR grade, Harrick Scientific Corporation, USA) was used as received, to prepare samples for DRIFTS measurements.

\subsection{MLD Experiments}

The MLD experiments were performed in a fluidized bed reactor operating at atmospheric pressure. The reactor included a glass column with an internal diameter of 2.5 $\mathrm{cm}$ and height of $25 \mathrm{~cm}$ placed on top of a Paja PTL 40/40-24 (Netherlands) vibration table, to enhance the fluidization of the silica NPs. In order to homogenize the gas flow through the column, and to avoid any powder loss from the top of the reactor, two stainless steel distributor plates were placed at the two ends of the column. The precursors were kept in separate stainless steel bubblers, under inert nitrogen atmosphere, and heated using a heating tape. PDIC is solid below $96{ }^{\circ} \mathrm{C}$; to increase the vapor pressure, the bubbler was heated to $110{ }^{\circ} \mathrm{C}$. The ED bubbler was kept at room temperature due to its high vapor pressure $\left(10 \mathrm{mmHg}\right.$ at $\left.20^{\circ} \mathrm{C}\right)$. Precursors were carried into the reactor by a nitrogen flow (99.999 $\mathrm{v} / \mathrm{v} \%$ ) passing through the bubblers. Three separate gas lines, two for precursor dosing and one for the nitrogen purge, were connected to the reactor. This arrangement ensures pure dosing of each precursor, and eliminates unwanted CVD reactions inside the lines. The lines were heated to and kept at $140{ }^{\circ} \mathrm{C}$ to prevent condensation and underdelivery of gaseous precursors. The reaction temperature in the column was set at $150{ }^{\circ} \mathrm{C}$ by means of a feedback-controlled infrared lamp, mounted parallel to the column. The bed 
temperature was monitored using a type-K thermocouple inserted into the bottom of the column. The exiting gas was sent from the top of the reactor through a series of washing bubblers, containing mineral oil (Kaydol) and sodium hydroxide $(\mathrm{NaOH})$ to capture unreacted precursors and process by-products. All the reactor constituents were contained inside a closed cabinet. In each experiment, $2 \mathrm{~g}$ of $\mathrm{SiO}_{2}$ powder was placed in the column. Each MLD cycle started with a PDIC pulse, followed by an ED pulse through the fluidized bed, with nitrogen purging in between and at the end. The pulse times were first optimized to reach surface saturation. The details of the optimization experiments are depicted in Table 7.1. After verifying the MLD saturation behavior, the growth of the deposited film was analyzed after 2, 5, 10 and 25 cycles using the optimal pulse times.

Table 7.1. Reaction conditions to verify the MLD growth of polyurea on silica nanoparticles

\begin{tabular}{|c|c|c|c|c|c|c|}
\hline Experiment & $\begin{array}{l}\text { PDIC pulse } \\
\text { time (min) }\end{array}$ & $\begin{array}{l}\mathrm{N}_{2} \text { purging } \\
\text { time (min) }\end{array}$ & $\begin{array}{c}\text { ED } \\
\text { pulse time } \\
\text { (min) }\end{array}$ & $\mathrm{T}_{\text {bubbler }}{ }^{\text {PDIC }}\left({ }^{\circ} \mathrm{C}\right)$ & $\mathrm{T}_{\text {bubbler }}{ }^{\mathrm{ED}}\left({ }^{\circ} \mathrm{C}\right)$ & $\mathrm{T}_{\text {reaction }}\left({ }^{\circ} \mathrm{C}\right)$ \\
\hline 1 & 0.5 & \multirow{10}{*}{5} & \multirow{5}{*}{2} & \multirow{10}{*}{110} & \multirow{10}{*}{30} & \multirow{10}{*}{150} \\
\hline 2 & 1 & & & & & \\
\hline 3 & 2 & & & & & \\
\hline 4 & 3 & & & & & \\
\hline 5 & 5 & & & & & \\
\hline 6 & \multirow{5}{*}{1} & & 0.5 & & & \\
\hline 7 & & & 1 & & & \\
\hline 8 & & & 2 & & & \\
\hline 9 & & & 3 & & & \\
\hline 10 & & & 5 & & & \\
\hline
\end{tabular}




\subsection{Thermogravimetric Analysis}

In order to verify the MLD growth and quantify the polyurea coating, Thermogravimetric Analysis (TGA) was performed using a TGA/SF1100 STARe system (Mettler Toledo, USA). The measurements were done by heating 2-3 mg of the fine powder from room temperature to $850{ }^{\circ} \mathrm{C}$ with a rate of $20^{\circ} \mathrm{C} / \mathrm{min}$ under air atmosphere, while monitoring the mass of the sample. We considered the mass loss in the temperature range of $200-350{ }^{\circ} \mathrm{C}$ to be related to the removal of polyurea chains (see Figure 7.2 ). With this assumption, one can calculate the polyurea loading $(v)$, from the TGA mass loss, using Equation 7-1:

$v\left(\frac{\text { urea monomer }}{n m^{2}}\right)=\frac{10^{3} \times N_{A}}{M_{W} \times S_{A}}\left(\frac{\Delta W}{100_{\text {g modified silica }}-\Delta W}\right)$

(Equation 7-1)

$v$ : Polyurea loading as the number of urea segments deposited per unit surface area

$\Delta W:$ TGA mass loss (g) between $200-350^{\circ} \mathrm{C}$

$M_{W}$ : Molecular weight of a single urea segment from one PDIC/ED cycle $(220 \mathrm{~g} / \mathrm{mol})$

$S_{A}$ : average surface area of the nanoparticles $\left(200 \mathrm{~m}^{2} / \mathrm{g}\right)$

$N_{A}:$ Avogadro constant

\subsection{DRIFTS}

To confirm the polyurea deposition and its self-limiting growth, Diffuse Reflectance IR Fourier Transform Spectroscopy (DRIFTS) was utilized according to what was described in Chapter 3.

\subsection{XPS Measurements}

X-ray Photoelectron Spectroscopy (XPS) was conducted according to what was described in Chapter 3. 


\subsection{EFTEM}

Transmission Electron Microscopy (TEM) with Energy-Filtering mode (EFTEM) was done on the silica nanoparticles, using a Philips CM300ST-FEG Transmission Electron Microscope in order to visualize the MLD deposited polyurea films on the silica.

\subsection{Computational Details}

Density Functional Theory (DFT) calculations were carried out on two model structures: a silica protoparticle with silanol groups on both sides, and an urea segment from the reaction between PDIC and ED, grafted to a silanol group. The models were prepared using GaussView 6, and the quantum mechanical calculations were done by the Gaussian 09W package ${ }^{23}$. The ground state geometry of each model at their minimum potential energy was calculated by solvation of the time-independent Schrödinger equation, utilizing the hybrid Becke three-parameter exchange correlation functional (B3LYP), with a split-valence double zeta basis set $6-31+G(d)$ to include both polarization functions and long range interactions ${ }^{24-26}$. All calculations were performed considering room temperature and in the presence of an external electric field of 0.0001 au ( 51.4 $\mathrm{kV} / \mathrm{mm}$ ). Total density of states (TDOS) and the band structure of the models were calculated by means of the multifunctional wavefunction analyzer Multiwfn ${ }^{27}$.

\section{Results and Discussion}

The key characteristic of an MLD process is the self-limiting, layer-by-layer growth of the organic film. We evaluated the possibility of depositing polyurea, in a self-saturating fashion, on silica nanoparticles. TGA and DRIFTS were utilized to verify this self-limiting behavior and to quantify the amount of deposited organic matter on the NPs. The TGA thermograms of the nanoparticles modified with different precursor pulse times, along with that of the untreated silica, are presented in Figure 7.2. 

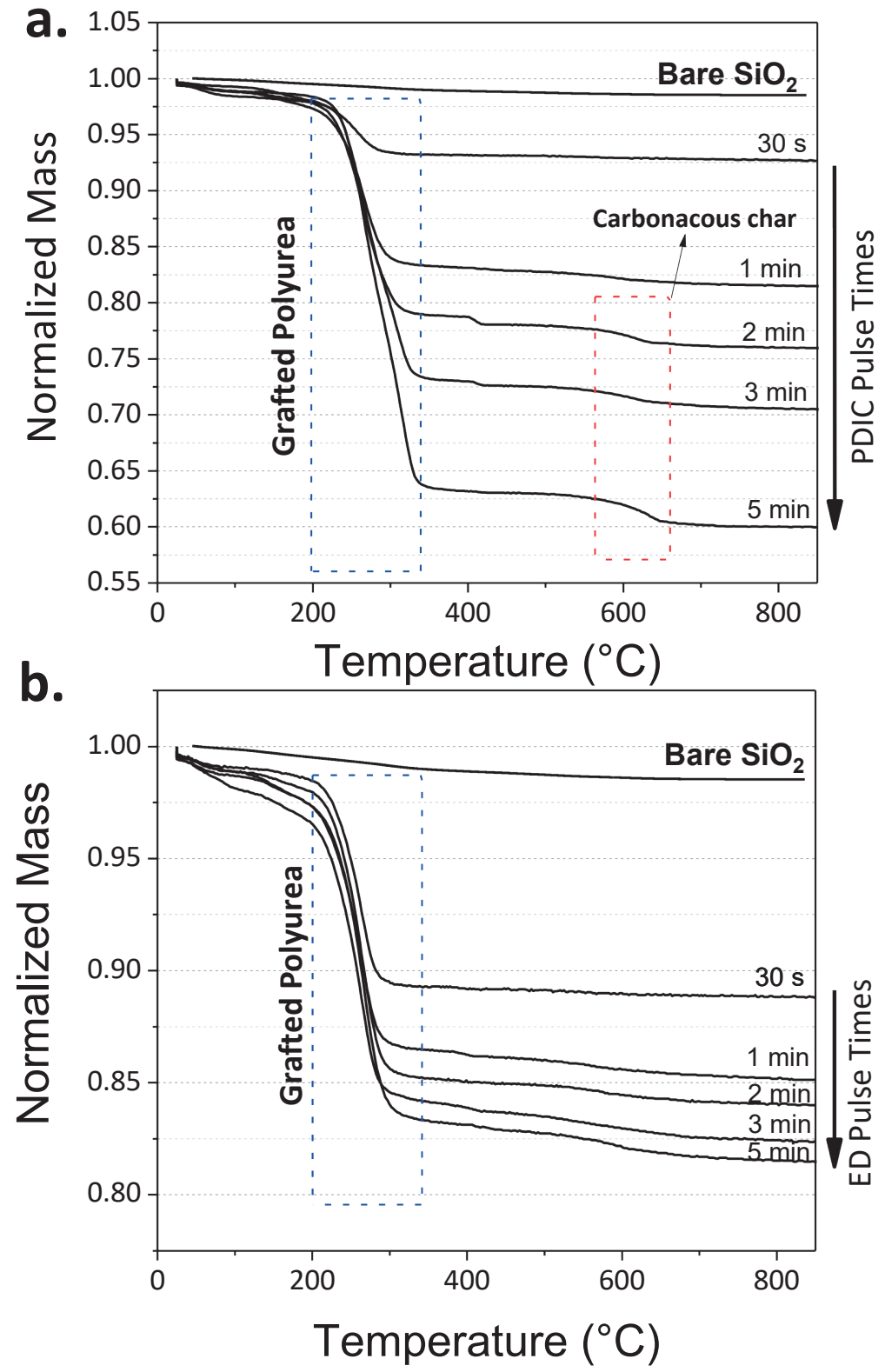

Figure 7.2. Thermograms of modified silica nanoparticles with different $a$ : PDIC and b: ED pulse times, alongside with the thermogram of the bare $\mathrm{SiO}_{2}$ nanoparticles 
The major mass loss between $200-350^{\circ} \mathrm{C}$ is related to the removal of polyurea chains from the NP surface. For PDIC pulse times longer than $1 \mathrm{~min}$, there is an additional mass loss step between $550-650^{\circ} \mathrm{C}$ related to the decomposition of carbonaceous char produced during the first decomposition step. This is more likely when there is an abundance of aromatic groups in the film due to the physisorption of PDIC, which is supported by the emergence of the isocyanate band at $2280 \mathrm{~cm}^{-1}$ in the DRIFTS spectrum after 5 minutes of PDIC pulse (Figure 7.4a). This also suggests that after $1 \mathrm{~min}$ of PDIC pulsing, the film growth may proceed via CVD reactions that prevent saturation. This is a common case for low vapor pressure "sticky" precursors such as PDIC, for which a longer purging time is required to remove the unreacted molecules from the reactor ${ }^{10,19,28}$. The deviation from saturation in Figure 7.3a can be, therefore, attributed to the physisorption of PDIC onto the NPs, affecting the mass loss measured by TGA. Nevertheless, the urea chains do exhibit a self-limiting growth with saturation time of 1 min for both precursors as seen in Figure $7.4 \mathrm{~b}$. This is confirmed by calculating the area under the amide I and amide II DRIFTS bands, characteristic to the urea units, emerging at $1650 \mathrm{~cm}^{-1}$ and $1510 \mathrm{~cm}^{-1}$, respectively. With the self-limiting behavior being confirmed, we verified the linear evolution of the polyurea MLD growth, using the dosing time of $1 \mathrm{~min}$ for both precursors. Separate experiments were done at 2, 5, 10 and 25 PDIC/ED cycles, and the results (Figure 7.3c) clearly indicate the linear growth of the polyurea films, with an approximate GPC of 0.5 segments per unit area. 
a.

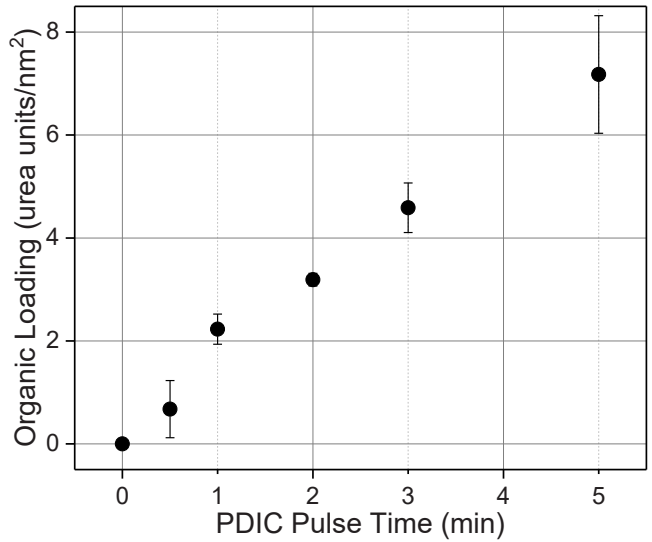

b.

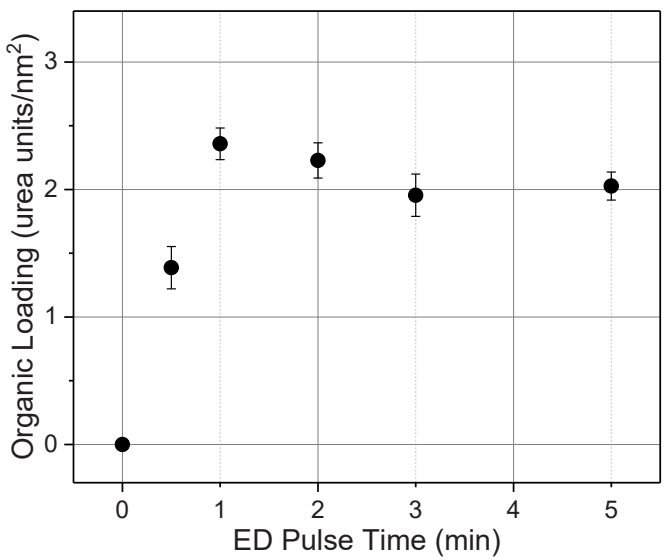

C.

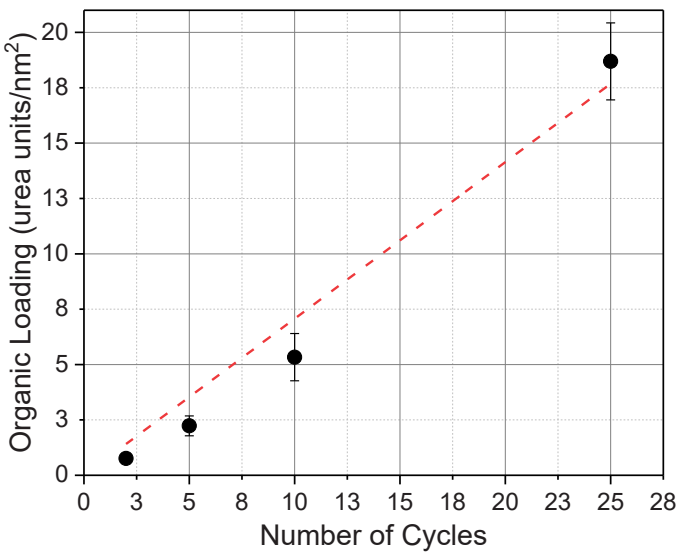

Figure 7.3. Polyurea film growth at different a: PDIC and b: ED pulse times, along with c: demonstration of the linear growth of the film at different number of cycles (The fitted line is to guide the eye). 
Figure 7.4c demonstrates the DRIFTS spectra for the polyurea-coated NPs in different cycles compared to the untreated silica and the DFT calculated spectrum. On account of computational practicality, the DFT calculations were performed on a single urea segment attached to a silanol group on silica. The amide I and amide II bands are characteristic to the urea segments, emerging at $1650 \mathrm{~cm}^{-1}$ and $1510 \mathrm{~cm}^{-1}$, respectively. All the bands related to the polyurea film, i.e. the amide I and amide II modes, the $\mathrm{N}-\mathrm{H}$ and $\mathrm{C}-\mathrm{H}$ stretch and the $\mathrm{CH}_{2}$ wagging increase significantly upon increasing the number of MLD cycles. At 25 cycles, a band emerges at $2280 \mathrm{~cm}^{-1}$ related to the isocyanate groups, and is indicative of unreacted PDIC molecules that were not purged from the reactor. This band is not observed at lower MLD cycles which implies that at least until 10 cycles, the PDIC has completely reacted to form urea segments. It is also noteworthy that the isolated silanol band at $3750 \mathrm{~cm}^{-1}$ completely vanishes after 10 MLD cycles, which is due to their chemical attachment to the urea groups. Comparing the experimental and the DFT calculated spectra, except for a few discrepancies due to the DFT model simplification, bands are in good accordance with the experimental spectra. These differences between the model and experimental spectra stem from the intramolecular interactions in the film. For instance, the $\mathrm{N}-\mathrm{H}$ stretching band observed at $3400 \mathrm{~cm}^{-1}$ in the calculated spectrum has been red-shifted and broadened in the experimental spectra. This is likely due to the different levels of hydrogen bonding between the $\mathrm{N}-\mathrm{H}$ groups and the carbonyl oxygen of the adjacent chains, which has also been reported in previous works on polyurea films ${ }^{19,29}$. 


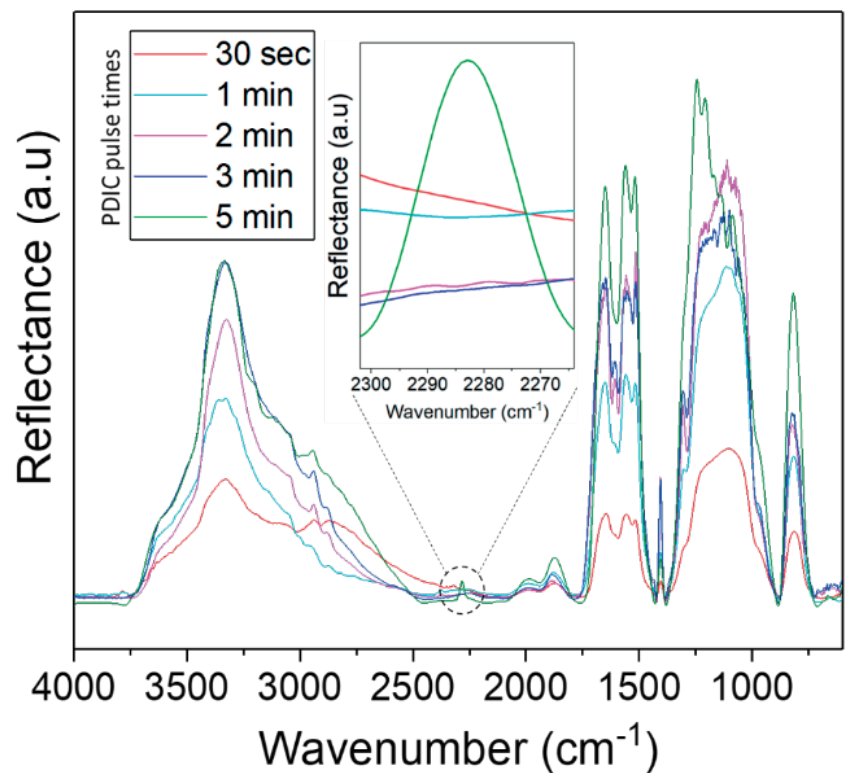

a.

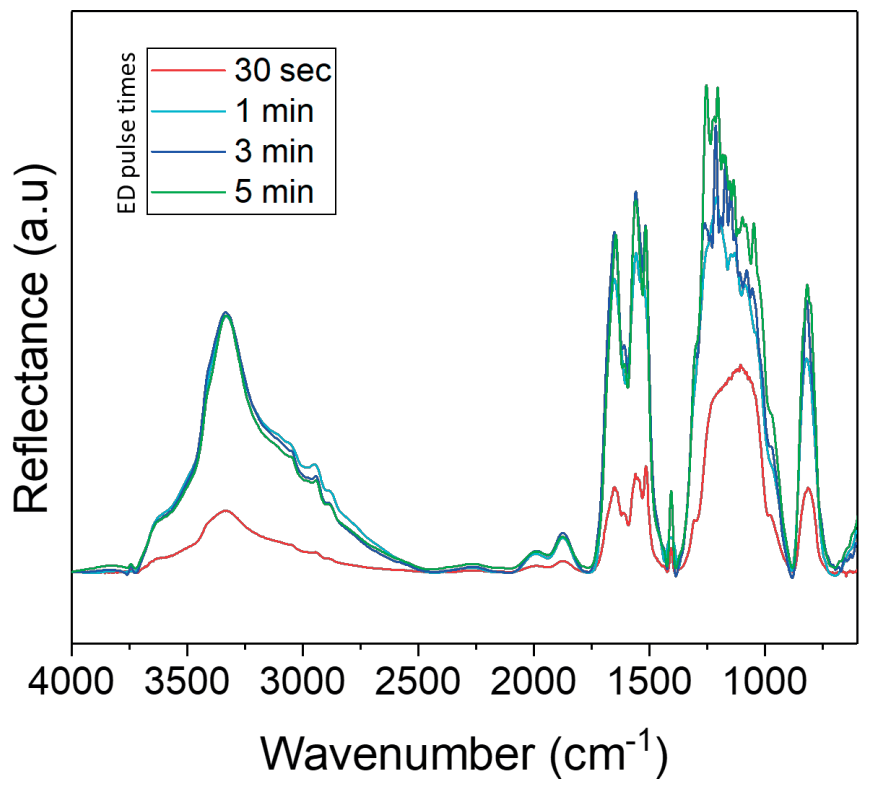


b.
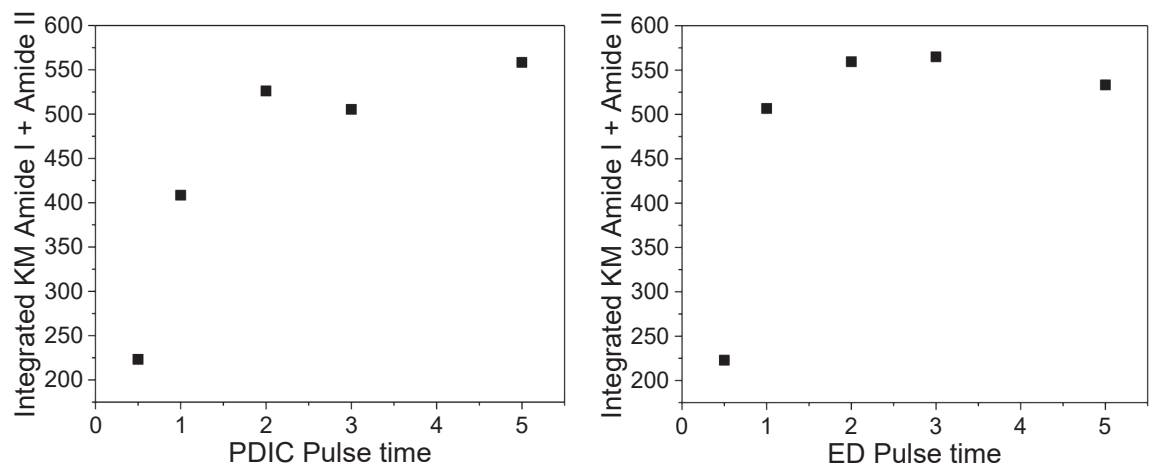

C.

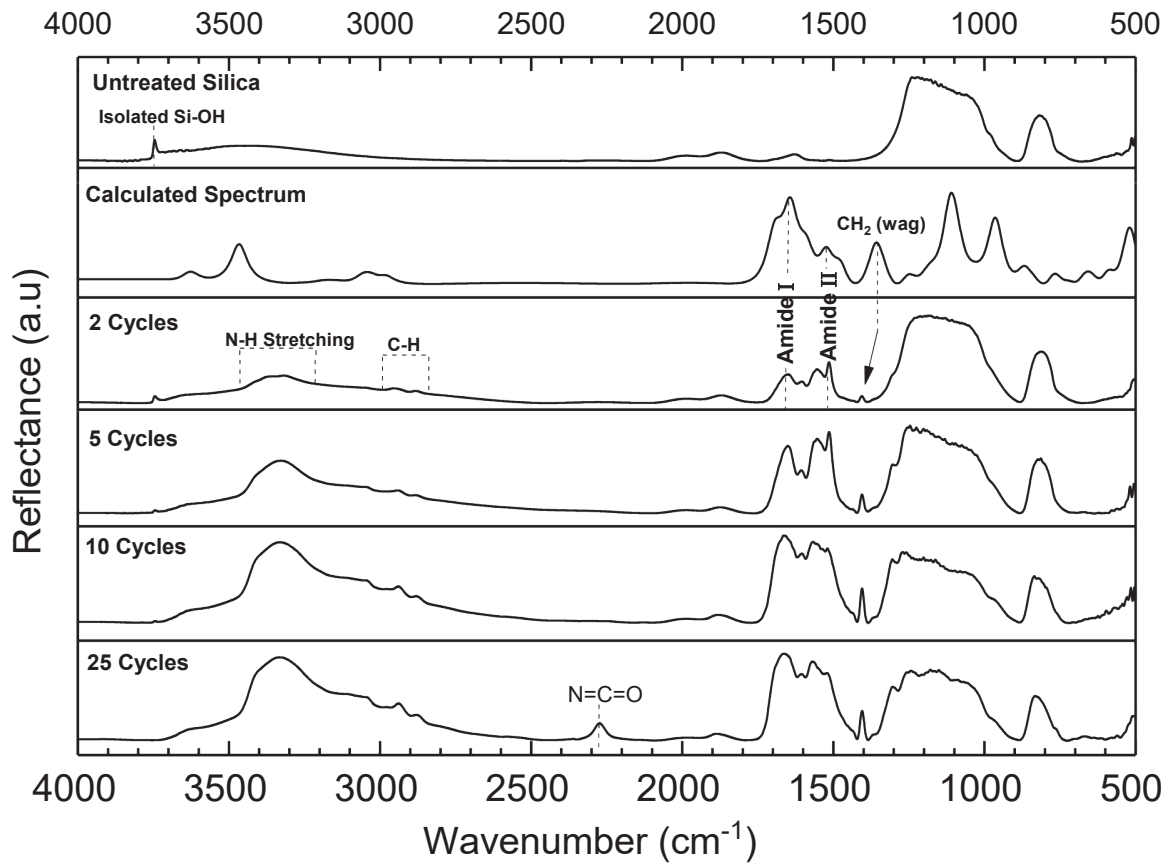

Figure 7.4. a: DRIFTS spectra for the saturation experiments with different precursor pulse times b: the integrated Kubelka-Munk (KM) amide I and amide II bands, and c: spectra of the polyurea coated NPs in different MLD cycles compared to the untreated silica and the DFT calculated spectrum

The XPS spectra of the modified silica after $10 \mathrm{MLD}$ cycles is compared to the bare $\mathrm{SiO}_{2}$ in Figure 7.5. The emergence of $\mathrm{N}(1 \mathrm{~s})$ and $\mathrm{C}(1 \mathrm{~s})$ bands for the treated nanoparticles in Figure 7.5a confirms the presence of the polyurea chains on their surface. The atomic percentages presented in Table 7.2 show the gradual increase of carbon and nitrogen contents with the number of cycles. For an ideal polyurea film from PDIC/ED, the stoichiometric C:N:O ratio 
should be 5:2:1. This ratio tends to be rich in oxygen for the MLD films with less than 10 cycles, which is due to the contribution of oxygen from the underlying $\mathrm{SiO}_{2}$.. For the 10 cycle sample, the $\mathrm{C}: \mathrm{N}: \mathrm{O}$ ratio reaches $4.7: 1.8: 1$, which signifies the close to ideal coverage of polyurea film on the nanoparticles after 10 cycles. The C:N ratio, however, is in good accordance with the theoretical ratio of 2.5 for an urea unit, regardless of the number of cycles. The elemental fine scans (Figures 7.5b, 7.5c and 7.5d) verify the chemical composition of the polyurea film. The $\mathrm{N}(1 \mathrm{~s})$ scan exhibits one single peak at $399.7 \mathrm{eV}$, representative of the $\mathrm{C}-\mathrm{N}$ bonds in the urea units. The $\mathrm{O}(1 \mathrm{~s})$ fine scan is de-convoluted into two peaks at $530.4 \mathrm{eV}$ and $532.5 \mathrm{eV}$, which are indicative of oxygen atoms in the carbonyl groups and silica, respectively. The $\mathrm{C}(1 \mathrm{~s})$ fine scan reveals three peaks: the one with the lowest binding energy at $284.4 \mathrm{eV}$ can be attributed to the carbons from the electron-rich aromatic groups. The peak at $285.7 \mathrm{eV}$ results from the alkyl carbons, and the highest binding energy peak at $288.5 \mathrm{eV}$ is indicative of carbons from the carbonyl groups. The theoretical ratio of the three types of carbon i.e. aromatic/alkyl/carbonyl in the urea segments is $3: 1: 1$, and our deposited film at 10 cycles exhibits a ratio of 2.1:1.1:1. The shortage of the aromatic carbons compared to the theoretical ratio is likely due to the growth of the urea groups from the aromatic rings ${ }^{19}$. The substituted carbons are, therefore, chemically shifted by the urea towards higher binding energies, which leads to the expected peak ratio to be $2: 2: 1$ rather than $3: 1: 1$. 
a.

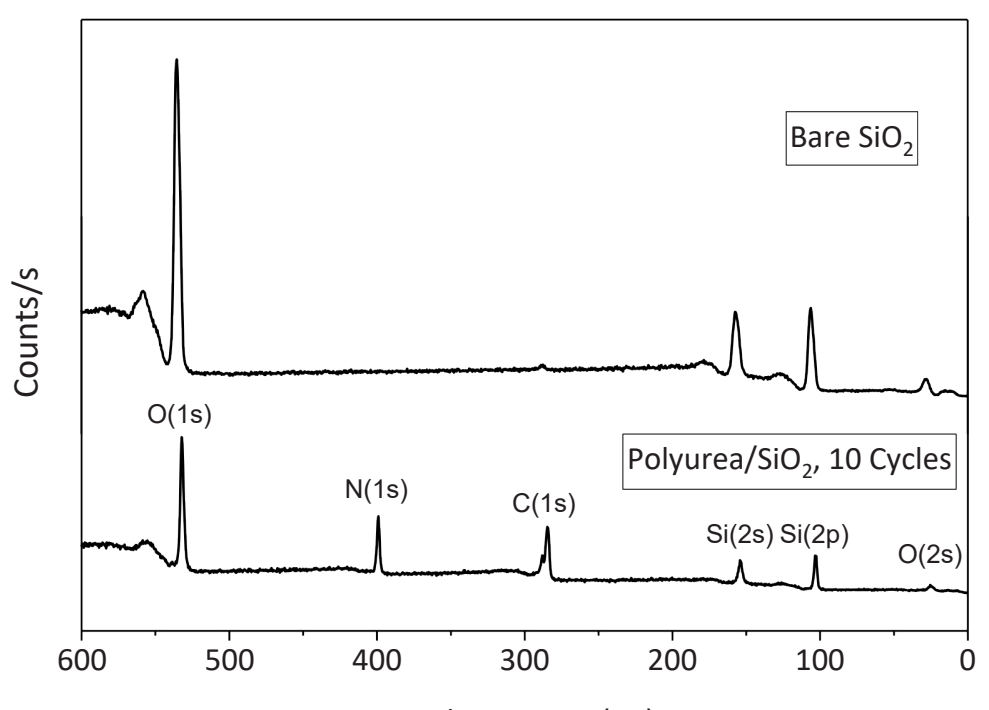

Binding Energy (eV)
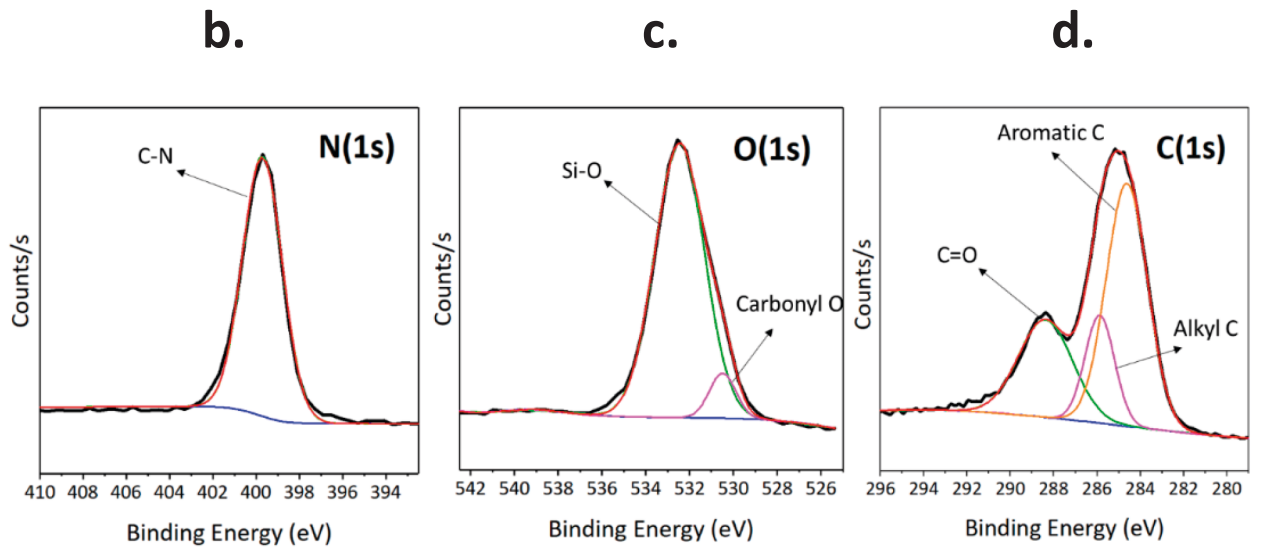

Figure 7.5. a: XPS full spectra of the NPs treated in 10 cycles compared to the untreated reference, $\mathrm{b}$ :, $\mathrm{c}$ : and $\mathrm{d}$ : the deconvoluted $\mathrm{N}(1 \mathrm{~s})$ and $\mathrm{O}(1 \mathrm{~s})$ and $\mathrm{C}(1 \mathrm{~s})$ elemental fine scans for the silica sample treated in 10 cycles 
Table 7.2. Atomic percentages (at \%) of $\mathrm{C}, \mathrm{N}, \mathrm{O}, \mathrm{C}: \mathrm{N}: \mathrm{O}$ and $\mathrm{C}: \mathrm{N}$ ratios for NPs treated at different number of cycles

\begin{tabular}{ccccccc}
\hline $\begin{array}{c}\text { Number of } \\
\text { MLD Cycles }\end{array}$ & C (at \%) & N (at \%) & O (at \%) & Si (at \%) & C:N:O & C:N \\
\hline 2 Cycles & $7.01 \pm 0.08$ & $2.81 \pm 0.23$ & $62.69 \pm 0.47$ & $27.38 \pm 0.42$ & $2.5: 1: 2.8$ & 2.49 \\
\hline 5 Cycles & $17.96 \pm 0.57$ & $7.33 \pm 0.21$ & $52.06 \pm 0.43$ & $22.61 \pm 0.03$ & $2.6: 1.07: 1$ & 2.45 \\
\hline 10 Cycles & $27.87 \pm 0.12$ & $11.71 \pm 0.82$ & $42.25 \pm 0.35$ & $18.16 \pm 0.82$ & $4.7: 1.8: 1$ & 2.38 \\
\hline 25 Cycles & $44.42 \pm 5.16$ & $17.79 \pm 2.17$ & $27.37 \pm 4.55$ & $10.41 \pm 2.8$ & $6.8: 2.7: 1$ & 2.5 \\
\hline
\end{tabular}

Figure 7.6 presents transmission electron microscopy (TEM) images visualizing the spatial distribution of the deposited MLD films on the nanoparticles. The primary particles are distinguishable in the fumed silica clusters, and the carbon mapped images show the deposited polyurea film on their surface. Increasing the number of cycles, in general, has resulted in more coverage of the surface as at 25 cycles very sharp carbon coated areas are observed on the nanoparticles. 


\section{Cycles}

a.

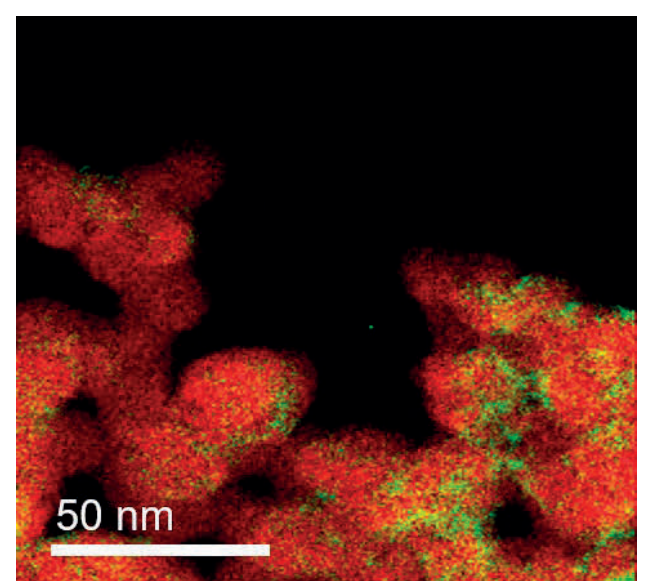

b.

\section{Cycles}
c.
d.

$50 \mathrm{~nm}$ 


\section{Cycles}

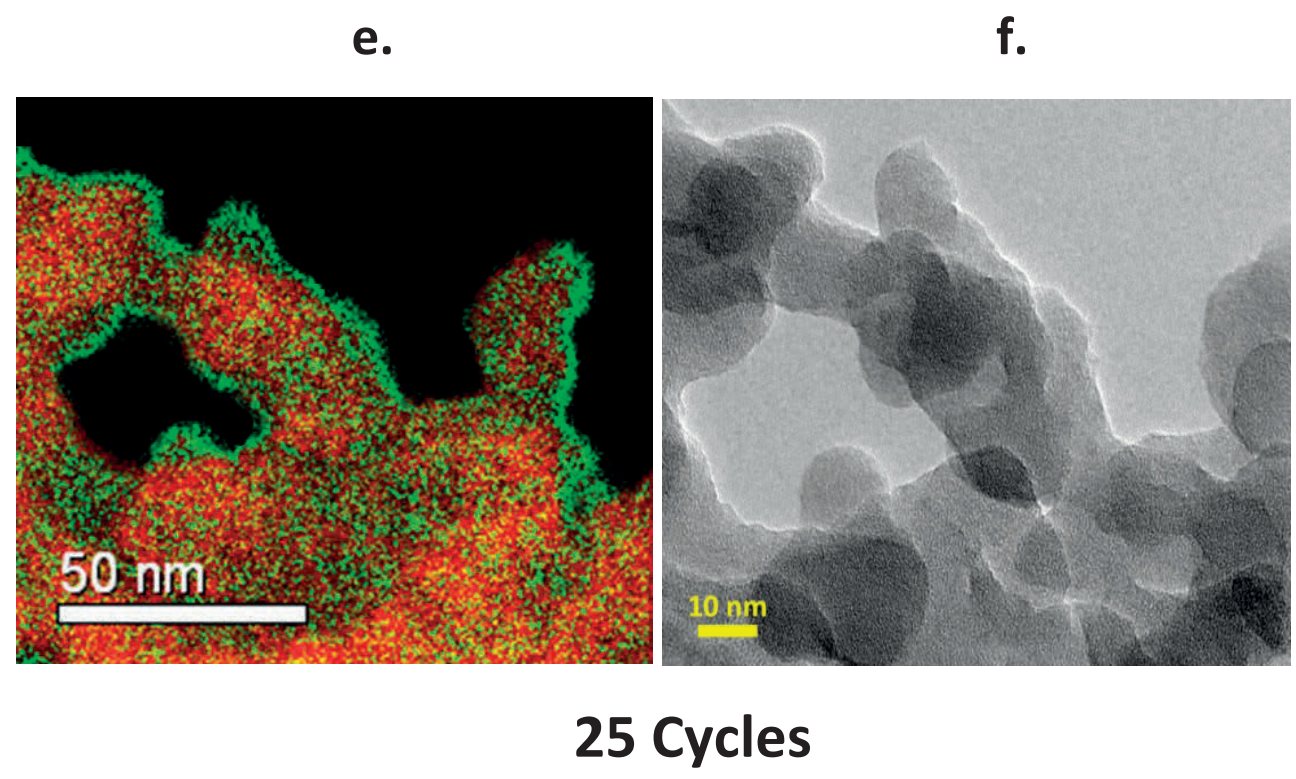

g.

h.
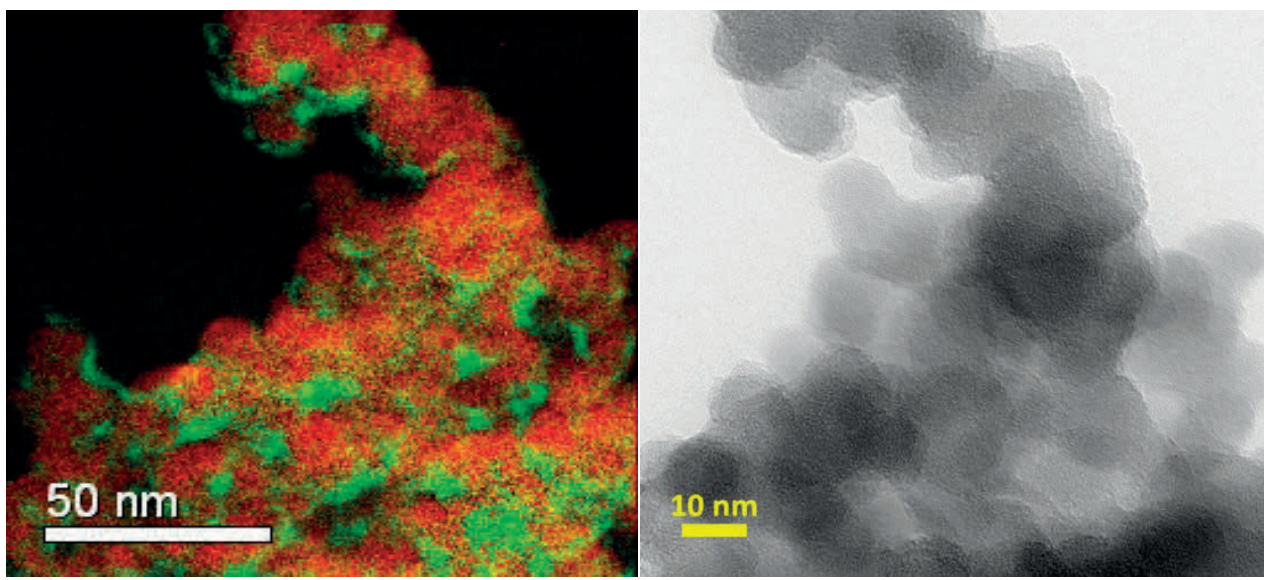

Figure 7.6. a, c, e, g: carbon and silicon mapping of the captured images (green: C, red: Si); b, d, f and h: TEM images of the NPs. 
The above results prove the feasibility of molecular layer deposition of polyurea on silica nanoparticles. The deposited urea units, containing amide and phenyl functional groups in their structure, are prone to introduce new localized states at the filler-polymer interfaces when dispersed in the nanocomposite. These localized states, depending on their depth and density can be of benefit in developing high performance insulating materials. The dielectric performance of the nanocomposites filled with urea-modified nanoparticles will be elaborated in the next chapter.

\section{Conclusions}

We have successfully demonstrated the successful deposition of polyurea films on the surface of fumed silica nanoparticles via molecular layer deposition (MLD), using phenyldiisocyanate (PDIC) and ethylenediamine (ED) as precursors. The key characteristics of an MLD process i.e. self-limiting behavior and linear film growth rate, were verified using TGA and DRIFTS. DRIFTS spectra demonstrate the characteristic bands of urea segments, well in-line with the DFT calculated spectrum, with increasing intensities with the number of MLD cycles. The atomic percentages measured by XPS show great accordance with the stoichiometric ratios, and three distinct types of carbon, namely aromatic, alkyl and carbonyl, are detected in the film, as expected. The resulted modified nanoparticles can find application in nanodielectrics as they are endowed with electrically active functional groups on their surface, and prone to introduce new localized states to the filler-polymer interfaces. 


\section{References}

[1] Brinker, C. J.; Scherer, G. W., Sol-gel science: the physics and chemistry of sol-gel processing Academic press: 2013;

[2] Alimardani, M.; Razzaghi-Kashani, M.; Karimi, R.; Mahtabani, A., Contribution Of Mechanical Engagement And Energetic Interaction In Reinforcement Of SBR-Silane Treated Silica Composites. Rubber Chemistry and Technology, 89 (2), 2016, pp 292-305.

[3] Vansant, E. F.; Van Der Voort, P.; Vrancken, K. C., Characterization and chemical modification of the silica surface Elsevier: 1995; Vol. 93.

[4] Lazghab, M.; Saleh, K.; Guigon, P., Functionalisation of porous silica powders in a fluidised-bed reactor with glycidoxypropyltrimethoxysilane (GPTMS) and aminopropyltriethoxysilane (APTES). Chemical engineering research and design, 88 (5-6), 2010, pp 686-692.

[5] Powell, Q. H.; Fotou, G. P.; Kodas, T. T.; Anderson, B. M.; Guo, Y., Gas-phase coating of TiO 2 with SiO 2 in a continuous flow hot-wall aerosol reactor. Journal of Materials Research, 12 (2), 1997, pp 552-559.

[6] Yoshimura, T.; Tatsuura, S.; Sotoyama, W., Polymer films formed with monolayer growth steps by molecular layer deposition. Applied Physics Letters, 59 (4), 1991, pp $482-$ 484.

[7] Van Bui, H.; Grillo, F.; Van Ommen, J., Atomic and molecular layer deposition: off the beaten track. Chemical Communications, 53 (1), 2017, pp 45-71.

[8] George, S. M.; Yoon, B.; Dameron, A. A., Surface Chemistry for Molecular Layer Deposition of Organic and Hybrid Organic-Inorganic Polymers. Accounts of Chemical Research, 42 (4), 2009, pp 498-508.

[9] Kao, C.-Y.; Yoo, J.-W.; Min, Y.; Epstein, A. J., Molecular Layer Deposition of an Organic-Based Magnetic Semiconducting Laminate. ACS Applied Materials \& Interfaces, 4 (1), 2012, pp 137-141.

[10] Du, Y.; George, S., Molecular layer deposition of nylon 66 films examined using in situ FTIR spectroscopy. The Journal of Physical Chemistry C, 111 (24), 2007, pp 8509-8517.

[11] Shao, H.-I.; Umemoto, S.; Kikutani, T.; Okui, N., Layer-by-layer polycondensation of nylon 66 by alternating vapour deposition polymerization. Polymer, 38 (2), 1997, pp 459462.

[12] Kubono, A.; Yuasa, N.; Shao, H.-L.; Umemoto, S.; Okui, N., In-situ study on alternating vapor deposition polymerization of alkyl polyamide with normal molecular orientation. Thin Solid Films, 289 (1-2), 1996, pp 107-111.

[13] Kim, A.; Filler, M. A.; Kim, S.; Bent, S. F., Layer-by-layer growth on Ge (100) via spontaneous urea coupling reactions. Journal of the American Chemical Society, 127 (16), 2005, pp 6123-6132.

[14] Putkonen, M.; Harjuoja, J.; Sajavaara, T.; Niinistö, L., Atomic layer deposition of polyimide thin films. Journal of Materials Chemistry, 17 (7), 2007, pp 664-669.

[15] La Zara, D.; Bailey, M. R.; Hagedoorn, P.-L.; Benz, D.; Quayle, M. J.; Folestad, S.; van Ommen, J. R., Sub-nanoscale Surface Engineering of TiO2 Nanoparticles by Molecular Layer Deposition of Poly(ethylene terephthalate) for Suppressing Photoactivity and Enhancing Dispersibility. ACS Applied Nano Materials, 2020. 
[16] Gil-Font, J.; Hatte, M.-A.; Bailey, M. R.; Navarrete, N.; Ventura-Espinosa, J.; Goulas, A.; La Zara, D.; van Ommen, J. R.; Mondragón, R.; Hernández, L., Improving heat transfer of stabilised thermal oil-based tin nanofluids using biosurfactant and molecular layer deposition. Applied Thermal Engineering, 178, 2020, p 115559.

[17] Liu, M.; Li, X.; Karuturi, S. K.; Tok, A. I. Y.; Fan, H. J., Atomic layer deposition for nanofabrication and interface engineering. Nanoscale, 4 (5), 2012, pp 1522-1528.

[18] Bergsman, D. S.; Closser, R. G.; Tassone, C. J.; Clemens, B. M.; Nordlund, D.; Bent, S. F., Effect of Backbone Chemistry on the Structure of Polyurea Films Deposited by Molecular Layer Deposition. Chemistry of Materials, 29 (3), 2017, pp 1192-1203.

[19] Loscutoff, P. W.; Zhou, H.; Clendenning, S. B.; Bent, S. F., Formation of organic nanoscale laminates and blends by molecular layer deposition. ACS Nano, 4 (1), 2010, pp 331-341.

[20] Prasittichai, C.; Zhou, H.; Bent, S. F., Area selective molecular layer deposition of polyurea films. ACS Applied Materials \& Interfaces, 5 (24), 2013, pp 13391-13396.

[21] Bergsman, D. S.; Closser, R. G.; Bent, S. F., Mechanistic Studies of Chain Termination and Monomer Absorption in Molecular Layer Deposition. Chemistry of Materials, 30 (15), 2018, pp 5087-5097.

[22] Nye, R. A.; Kelliher, A. P.; Gaskins, J. T.; Hopkins, P. E.; Parsons, G. N., Understanding Molecular Layer Deposition Growth Mechanisms in Polyurea via Picosecond Acoustics Analysis. Chemistry of Materials, 32 (4), 2020, pp 1553-1563.

[23] M. J. Frisch, G. W. T., H. B. Schlegel, G. E. Scuseria, M. A. Robb, J. R. Cheeseman, G. Scalmani, V. Barone, G. A. Petersson, H. Nakatsuji, X. Li, M. Caricato, A. Marenich, J. Bloino, B. G. Janesko, R. Gomperts, B. Mennucci, H. P. Hratchian, J. V. Ortiz, A. F. Izmaylov, J. L. Sonnenberg, D. Williams-Young, F. Ding, F. Lipparini, F. Egidi, J. Goings, B. Peng, A. Petrone, T. Henderson, D. Ranasinghe, V. G. Zakrzewski, J. Gao, N. Rega, G. Zheng, W. Liang, M. Hada, M. Ehara, K. Toyota, R. Fukuda, J. Hasegawa, M. Ishida, T. Nakajima, Y. Honda, O. Kitao, H. Nakai, T. Vreven, K. Throssell, J. A. Montgomery, Jr., J. E. Peralta, F. Ogliaro, M. Bearpark, J. J. Heyd, E. Brothers, K. N. Kudin, V. N. Staroverov, T. Keith, R. Kobayashi, J. Normand, K. Raghavachari, A. Rendell, J. C. Burant, S. S. Iyengar, J. Tomasi, M. Cossi, J. M. Millam, M. Klene, C. Adamo, R. Cammi, J. W. Ochterski, R. L. Martin, K. Morokuma, O. Farkas, J. B. Foresman, and D. J. Fox, Gaussian 09, Revision A.02. Gaussian, Inc., 2016.

[24] Wu, C.; Wang, J.; Chang, P.; Cheng, H.; Yu, Y.; Wu, Z.; Dong, D.; Zhao, F., Polyureas from diamines and carbon dioxide: synthesis, structures and properties. Physical Chemistry Chemical Physics, 14 (2), 2012, pp 464-468.

[25] Becke, A. D., A new mixing of Hartree-Fock and local density-functional theories. The Journal of chemical physics, 98 (2), 1993, pp 1372-1377.

[26] Pritchard, B. P.; Altarawy, D.; Didier, B.; Gibson, T. D.; Windus, T. L., New Basis Set Exchange: An Open, Up-to-Date Resource for the Molecular Sciences Community. Journal of Chemical Information and Modeling, 59 (11), 2019, pp 4814-4820.

[27] Lu, T.; Chen, F., Multiwfn: A multifunctional wavefunction analyzer. Journal of Computational Chemistry, 33 (5), 2012, pp 580-592.

[28] Adamczyk, N.; Dameron, A.; George, S., Molecular layer deposition of poly (pphenylene terephthalamide) films using terephthaloyl chloride and p-phenylenediamine. Langmuir, 24 (5), 2008, pp 2081-2089. 
[29] Coleman, M. M.; Sobkowiak, M.; Pehlert, G. J.; Painter, P. C.; Iqbal, T., Infrared temperature studies of a simple polyurea. Macromolecular Chemistry and Physics, 198 (1), 1997, pp 117-136. 


\section{CHAPTER 8}

Dielectric properties of Nanocomposites:

Effect of Molecular Layer Deposition of Polyurea on Silica Nanoparticles 


\begin{abstract}
Grafting electrically active functional groups to the surface of nanoparticles (NPs) in a controlled manner can yield reproducible alterations in charge trapping and transport as well as space charge phenomena in nanodielectrics. In the following study, fumed silica NPs modified with polyurea via molecular layer deposition (MLD) are incorporated into a polymer blend based on polypropylene (PP)/ ethylene-octene-copolymer (EOC), and their morphological and dielectric properties are investigated. We demonstrate the alterations in the electronic structure of silica upon depositing urea units, using density functional theory (DFT) calculations. Subsequently, the effect of urea functionalization on the dielectric properties of nanocomposites is studied using thermally stimulated depolarization current (TSDC) and broadband dielectric spectroscopy (BDS) methods. The DFT calculations reveal the contribution of both shallow and deep traps upon deposition of polyurea onto the NPs. It could be concluded that the deposition of polyurea on nanoparticles results in a bi-modal distribution of trap depths that are related to each monomer in the urea units, and can lead to a reduction of space charge formation at fillerpolymer interfaces.
\end{abstract}




\section{Introduction}

Molecular layer deposition (MLD) is a robust technique to deposit organic films on flat surfaces as well as nanoparticles (NPs) ${ }^{1-5}$. As opposed to the conventional chemical vapor deposition (CVD), MLD utilizes self-limiting reactions between bi-functional precursors to grow organic films on the solid surface in a controlled layer-by-layer fashion, by dosing sequential pulses of each precursor into the reactor ${ }^{6}$. In Chapter 7, fumed silica NPs with a primary particle size of 7-50 $\mathrm{nm}$ were modified via MLD in a fluidized bed. Phenyl diisocyanate (PDIC) and ethylene diamine (ED) were used as precursors to deposit conformal films of polyurea on gram-scale silica NPs, for the first time. Such urea units contain phenyl and amide functional groups, whose deposition can be controlled by MLD down to the molecular scale. The presence of these functional groups on the NPs is expected to create new localized states (traps) with different energy levels by altering the electronic structure of the silica/polymer interfaces. Shallow traps, with energy levels generally well below $1 \mathrm{eV}$, can significantly hamper the formation of permanent space charge in the material as they would assist the transport of charge carriers and prevent them from accumulation ${ }^{7}$. Deep traps, with energies above $1 \mathrm{eV}$, can effectively immobilize charge carriers, reduce the hopping conduction of electrons and cause the formation of homocharge near the electrodes ${ }^{8}$. Therefore, MLD of polyurea films has the potential to control the macroscopic dielectric properties of the corresponding NCs.

In this chapter, fumed silica NPs modified with polyurea via MLD are incorporated into the model polymer blend system based on polypropylene (PP)/ ethylene-octene-copolymer (EOC), and their dielectric properties are investigated. Firstly, we demonstrate the alterations in the electronic structure of silica upon depositing urea units using density functional theory (DFT) calculations. The NP dispersion quality in the polymer matrix and the crystallization behavior of the NCs are analyzed by scanning electron microscopy (SEM) and differential scanning calorimetry (DSC). Subsequently, the effect of urea functionalization on the charge trapping and transport under a direct current (DC) electric field is studied using thermally stimulated depolarization current (TSDC) method. In addition, in order to further analyze the relaxation processes and the dielectric response of 
the nanocomposites (NCs), broadband dielectric spectroscopy (BDS) is performed on the NCs, and the results are discussed.

\section{Experimental Work}

\subsection{Silica Modification by Molecular Layer Deposition}

Silica (AEROSIL 200, Evonik Ind., Germany) treatment was carried out via molecular layer deposition (MLD) in a fluidized bed reactor according to what was described in Chapter 7.

\subsection{Preparation of the Nanocomposites}

These details can be found in Chapter 3.

\subsection{Morphology and Crystallinity Analysis}

Scanning Electron Microscopy (SEM) and the subsequent image analysis were performed in accordance with the description given in Chapter 4. Differential Scanning Calorimetry (DSC) was performed by means of a DSC 2500 (TA Instruments, USA). Specimens were subjected to two heating/cooling cycles from $-70^{\circ} \mathrm{C}$ to $200^{\circ} \mathrm{C}$ in nitrogen atmosphere. The heating/cooling rate was set at $3^{\circ} \mathrm{C} / \mathrm{min}$, in order to match the heating rate in TSDC measurements.

\subsection{Thermally Stimulated Depolarization Current (TSDC)}

Details of the Thermally Stimulated Depolarization Current (TSDC) measurements were described in Chapter 3.

\subsection{Broadband Dielectric Spectroscopy (BDS)}

Details of these measurements can be found in Chapter 3.

\subsection{Computational Details}

Density Functional Theory (DFT) calculations were carried out according to the description in Chapter 7. 


\section{Results and Discussion}

Grafting electrically active functional groups onto the surface of NPs would potentially alter the electronic structure of the surface groups and create localized states at the fillerpolymer interfaces. To demonstrate these alterations upon grafting urea films to the silica surface, DFT calculations were performed on a model structure of an urea unit coupled to a silanol group (see Figure 8.1a). Figures $8.1 \mathrm{~b}$ and $8.1 \mathrm{c}$ demonstrate the distribution of the highest occupied molecular orbitals (HOMO: -7.05 eV) and lowest unoccupied molecular orbitals (LUMO: $0.86 \mathrm{eV}$ ) of this structure, respectively. It is evident that the HOMO are mostly located on the second monomer ethylene diamine (ED), whereas the LUMO are distributed over the first monomer phenyl diisocyanate (PDIC) as well as the dangling silanol groups in the model. This suggests, on the one hand, that the silanol groups on the silica surface can noticeably contribute to the density of unoccupied states, i.e. it is rather likely to find conducting electrons around them. Therefore, grafting other moieties to cover these surface groups in order to control conductivity and space charge phenomena in the final NC appears very relevant. On the other hand, between the two monomers in the urea repeating unit, PDIC is more susceptible to contribute to the conduction processes in the presence of an electric field, likely by creating shallow trapping states. Moreover, the presence of the HOMO around the ED monomer can be beneficial in hampering the formation of space charge in the presence of the DC electric field. This is similar to what we observed for amino-modified silica (Chapter 6), where the amino group's large density of occupied states hindered the formation of space charge at the silica-polymer interfaces.

The total density of states (TDOS) at different energy levels for the bare as well as the urea modified silica are depicted in Figure 8.2. For the unmodified silica, a rather large density of hole traps is observed at the tail of the valence band, inside the band gap. Furthermore, at the tail of the conduction band, there is a distribution of relatively shallow electron traps. By introducing the phenyl and amide groups to the silica, both valence and conduction band extrema shift to higher energy levels, and the band gap becomes slightly $(0.84 \mathrm{eV})$ broader. The density of electron traps is larger for the urea modified silica, implying that new localized states are created as a result of this modification. These states 
are extended over $1.4 \mathrm{eV}$ below the conduction band, suggesting that there are contributions of both shallow and deep traps from this urea unit. The density of conduction states is significantly lower for the urea modified silica, which suggests that the conducting electrons are less likely to be drifting in the conduction band, when this urea unit is present on the silica surface.

a.

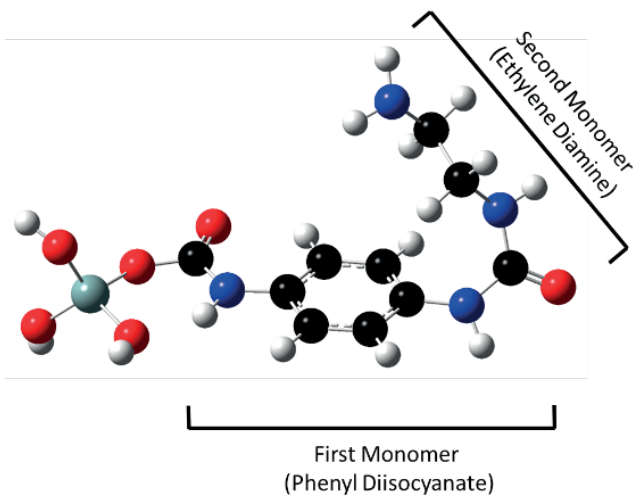

b.

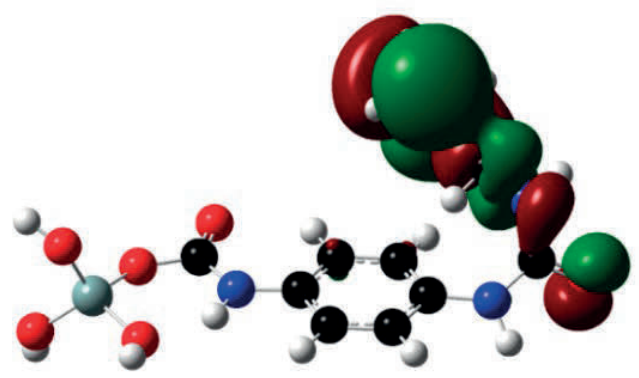

C.

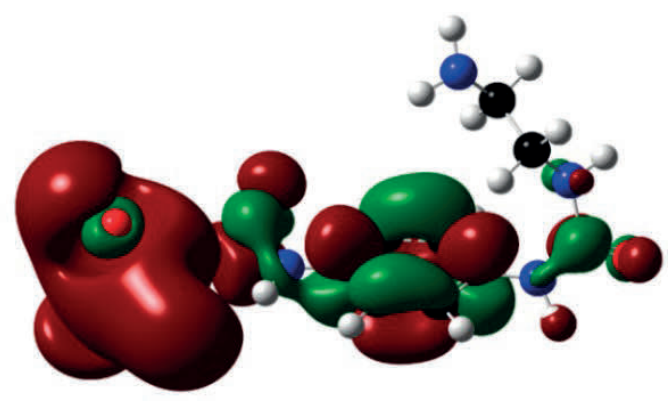

Figure 8.1. a: The optimized geometry of an urea unit made of phenyl diisocyanate and ethylene diamine grafted to a silanol group on silica; b: Highest occupied molecular orbitals (HOMO) and c: lowest unoccupied molecular orbitals (LUMO) visualized for the modified silica (gray: silicon, lightred: oxygen, black: carbon, blue: nitrogen and white: hydrogen; the dark-red and green color of the MOs are related to the positive and negative wavefunctions, respectively. 


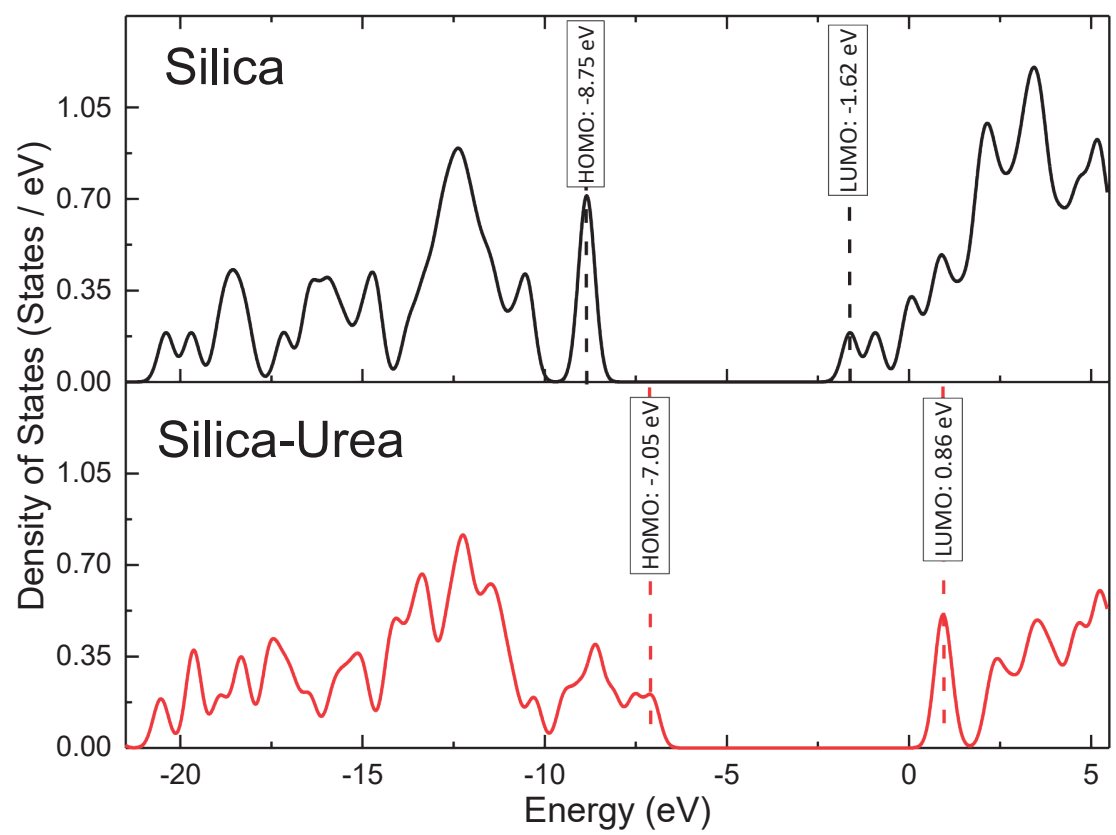

Figure 8.2. Total density of states (TDOS) for the urea-silica structure compared to the neat silica

Using DFT calculations, we have shown that the presence of urea units consisting of phenyl diisocyanate and ethylene diamine on the silica surface may introduce new localized states and alter the electronic structure of the filler surface. To demonstrate this experimentally, molecular layer deposition (MLD) is utilized to deposit homogenous polyurea films on the silica NPs in a controlled manner by cyclic pulsing of the two precursors in vapor phase into a fluidized bed. Each MLD cycle consists of 1 minute of respective PDIC and ED pulses, divided by 5 minutes of nitrogen purging to ensure selflimiting growth of the deposited film and limit CVD reactions in the powder bed. Details of the MLD processes, and the characterization of the functionalized NPs are reported Chapter 7. The polyurea films were deposited on silica NPs for 2, 5, 10 and 25 MLD cycles, in separate experiments. Figure 8.3 displays the linear growth of the polyurea loading upon increasing the number of cycles, with an approximate growth per cycle (GPC) of 0.7 urea units per $\mathrm{nm}^{2}$. 


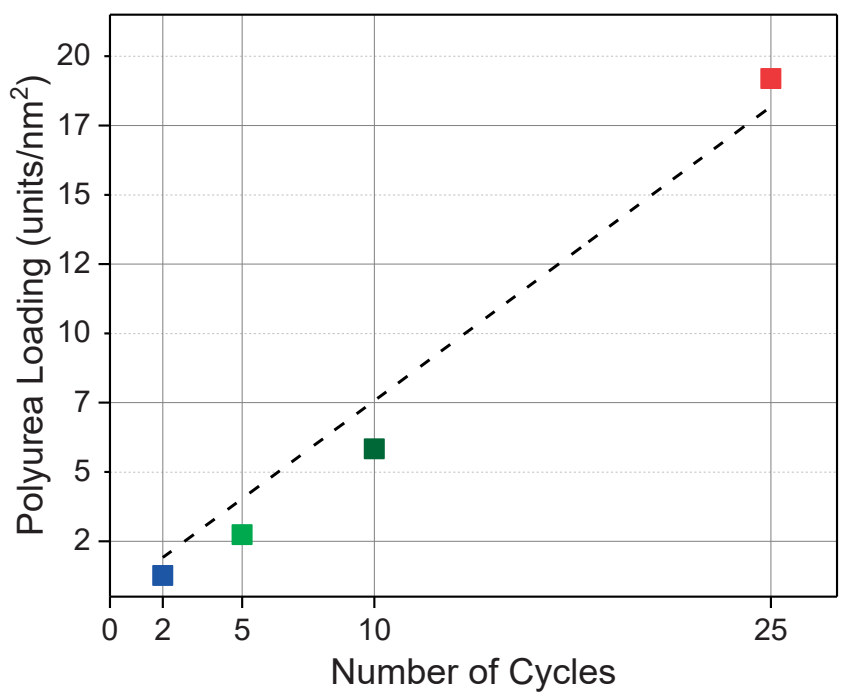

Figure 8.3. Evolution of the polyurea loading on NPs as a function of the number of MLD cycles.

Surface characteristics of the NPs can directly affect their nucleating ability in the polymer matrix, and hence the crystalline structure of the NCs. Consequently, this can influence the dielectric response of the material. Therefore, differential scanning calorimetry (DSC) was performed to analyze and compare the crystallization behavior of the NCs. Figures $8.4 \mathrm{a}$ and $8.4 \mathrm{~b}$ represent the melting and crystallization curves for the unfilled blend, and NCs with and without filler functionalization. The DSC spectra of the blend consist of four distinct peaks: two endothermic peaks $\left(108{ }^{\circ} \mathrm{C}\right.$ and $\left.148{ }^{\circ} \mathrm{C}\right)$ and two exothermic peaks $\left(97^{\circ} \mathrm{C}\right.$ and $115^{\circ} \mathrm{C}$ ) corresponding to the melting and crystallization temperatures of the EOC and PP domains, respectively. The melting curves (Figure 8.4a), on the one hand, exhibit no significant changes upon incorporation of the silica and functionalization of it. On the other hand, the onset of crystallization tends to decrease by $3{ }^{\circ} \mathrm{C}$, when the bare NPs are added to the system. This is despite the expected nucleating effect of NPs in composite systems, and is likely due to the adsorption of polar antioxidants onto the silica surface, reducing heterogeneous nucleation during the cooling step ${ }^{9-11}$. Nevertheless, the modified NCs exhibit crystallization onsets similar to that of the unfilled blend. This implies that the polyurea deposition facilitates nucleation, likely by reducing the adsorption of antioxidant particles onto the silica surface. Moreover, all the MLD modified 
samples exhibit similar crystallization curves indicating that the amount of deposited polyurea has no significant effect on the nucleation and crystal growth in the NCs. The degree of crystallization for each sample is calculated from the enthalpy of melting and presented in Table 8.1. It is evident that the addition of NPs and their modification, does not influence the overall degree of crystallization in the NCs. Accordingly, it can be assumed that any discrepancy in the dielectric response of the studied NCs would not be influenced by the differences in crystallization behavior of the materials.

a.

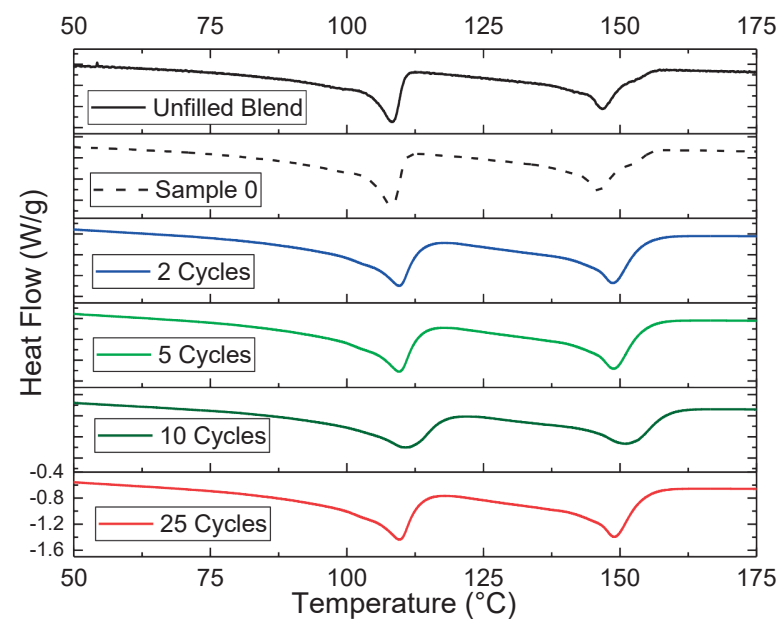

b.

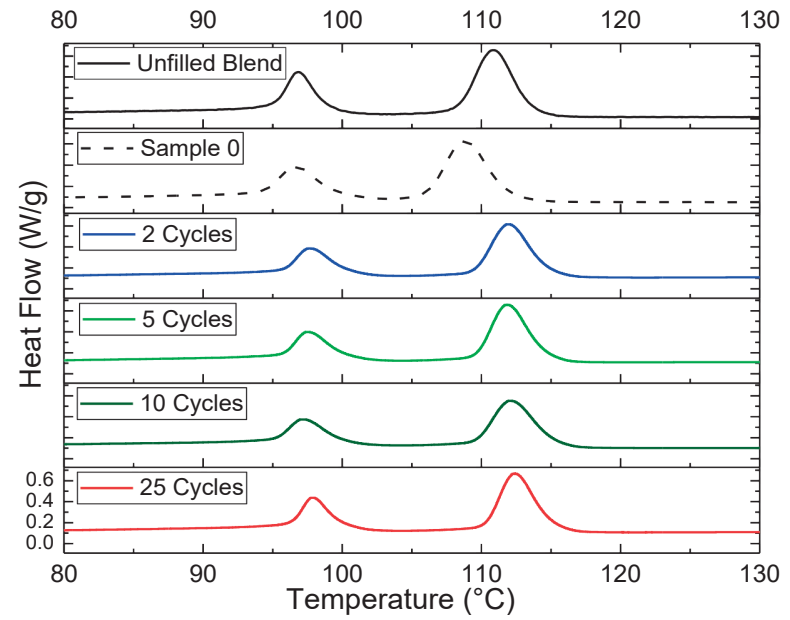

Figure 8.4. a: Melting and b: crystallization behavior of the studied NCs compared to the unfilled blend 
Table 8.1. DSC parameters of all studied samples

\begin{tabular}{cccc} 
& $\begin{array}{c}\text { Onset of } \\
\text { Crystallization }\left({ }^{\circ} \mathrm{C}\right)\end{array}$ & $\begin{array}{c}\text { Enthalpy of } \\
\text { Melting }(\mathrm{J} / \mathrm{g})\end{array}$ & $\begin{array}{c}\text { Percentage of } \\
\text { Crystallinity (\%) }\end{array}$ \\
\hline Unfilled Blend & 115 & 77.3 & 31 \\
Sample 0 & 112 & 74.9 & 30 \\
2 Cycles & 114 & 72.1 & 29 \\
5 Cycles & 115 & 75.1 & 30 \\
10 Cycles & 115 & 71.6 & 29 \\
25 Cycles & 115 & 73.2 & 29
\end{tabular}

In order to analyze the dispersion of NPs in the polymer matrix, SEM was performed on the NCs, and the results are presented in Figure 8.5. In order to achieve a higher resolution and visualize the particles more clearly, SEM was performed on these samples with and without gold sputtering. In case of the sputtered samples, however, the morphological details of the polymer matrix were masked. Therefore, we present the image of the 5 cycles sample without sputtering. It can be observed that the PP/EOC blend exhibits a two-phase morphology, with the EOC domains elongated in the direction of the flow in the mold which is typical for this type of blend ${ }^{9}$. The mean aggregate size of the untreated silica NPs was reported to be around $300 \mathrm{~nm}$ in PP/EOC blends ${ }^{9}$. Upon increasing the number of MLD cycles, the mean aggregate size in the NCs reduces, reaching $160 \mathrm{~nm}$ for samples with 10 and 25 MLD cycles. This indicates that this modification has effectively reduced the energetic interactions between silica particles, resulting in a relatively enhanced dispersion of NPs throughout the polymer matrix. 

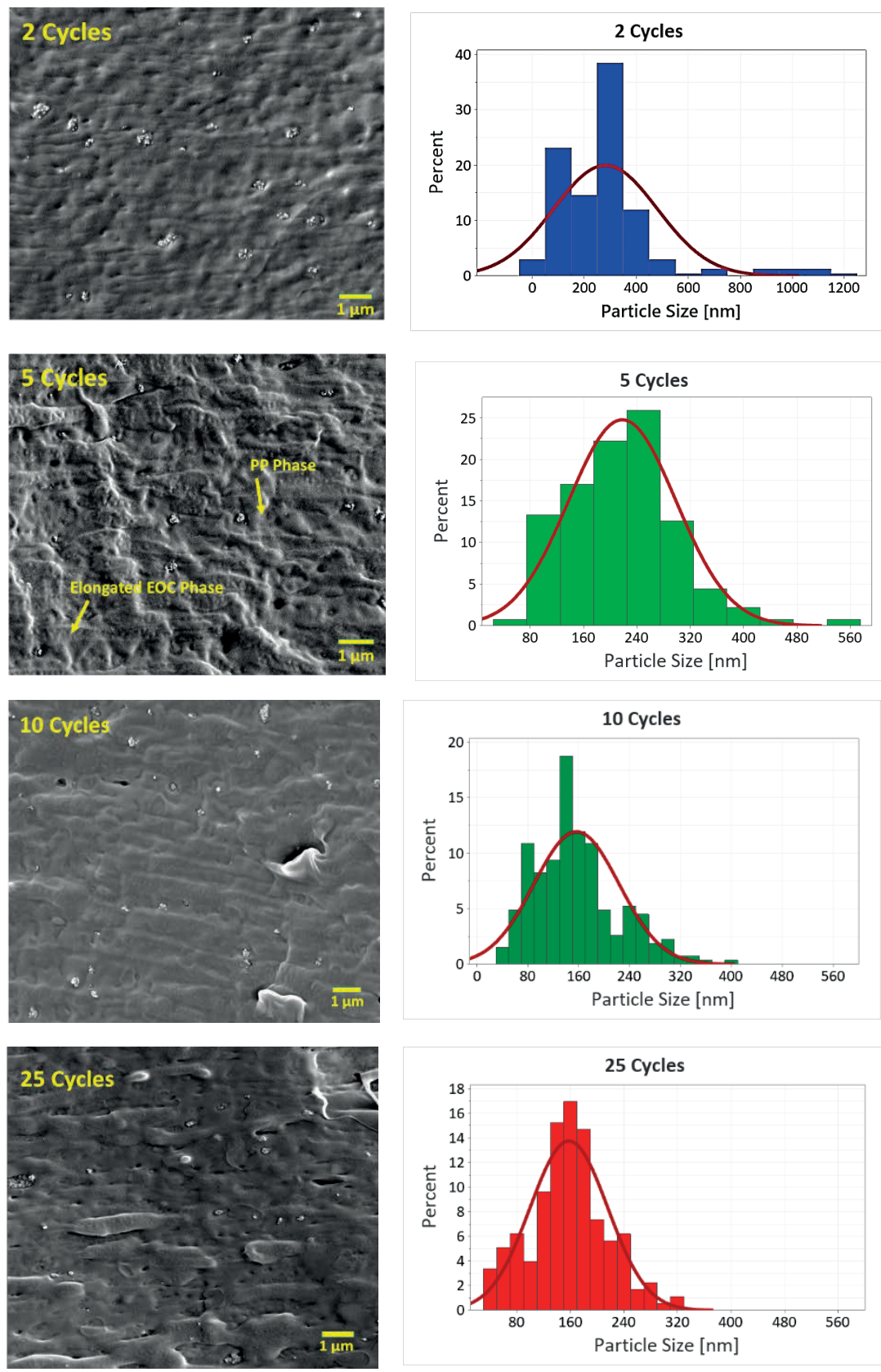

Figure 8.5. SEM images of NCs containing MLD modified silica, along with the particle size histograms; the image of the 5 cycles sample is taken without gold sputtering, in order to visualize the phase distribution of the polymer blend. 
Broadband dielectric spectroscopy (BDS) was performed in order to investigate the relaxation processes in the NC systems in the presence of an alternating electric field. These relaxation processes, depending on the frequency range, can then be attributed to dielectric phenomena such as polarization and space charge formation. Figure 8.6 presents the variations of the real $\left(\varepsilon_{r}{ }^{\prime}\right)$ and imaginary $\left(\varepsilon_{r}{ }^{\prime \prime}\right)$ parts of complex permittivity with respect to the frequency of the applied field. At frequencies higher than $1 \mathrm{~Hz}$, the unfilled blend and Sample 0 show little difference in the values of real permittivity. It is also clear that the modified NCs exhibit lower values of $\varepsilon_{r}^{\prime}$ at all frequencies, compared to the two references. Since real permittivity is related to the number of polarizable species in the system, the NC with 2-cycle-modified silica, with the lowest grafting density of polyurea, exhibits the lowest real permittivity among all MLD modified samples. $\varepsilon_{r}{ }^{\prime \prime}$ remains constant for all the samples at higher frequencies. At low frequencies (generally less than $1 \mathrm{~Hz}$ ), however, there is a steep increase in both parts of permittivity, indicating either the relaxation of large dipolar species, or the presence of Maxwell-Wagner space charge relaxations ${ }^{12-13}$. On the one hand, it is clear that these relaxations are somewhat suppressed when the NPs are incorporated into the system. On the other hand, deposition of polyurea on the NPs has resulted in further suppression of this low frequency variation. This indicates that the presence of polyurea films on the NPs has significant effects on the polarization processes and space charge formation in the corresponding NCs. This is well in-line with the DFT predictions: the reduced space charge relaxations in the modified NCs can be attributed to either the presence of shallow traps introduced by the phenyl rings in the polyurea film, or the amide groups' large density of valence states hampering the formation of space charge at the fillerpolymer interfaces. 
a.

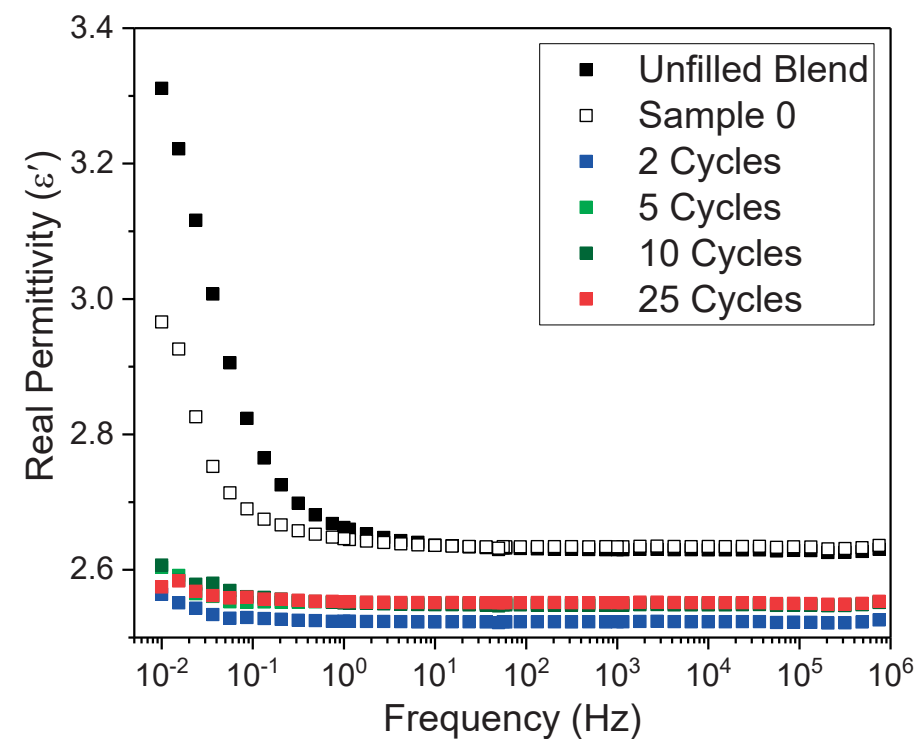

b.

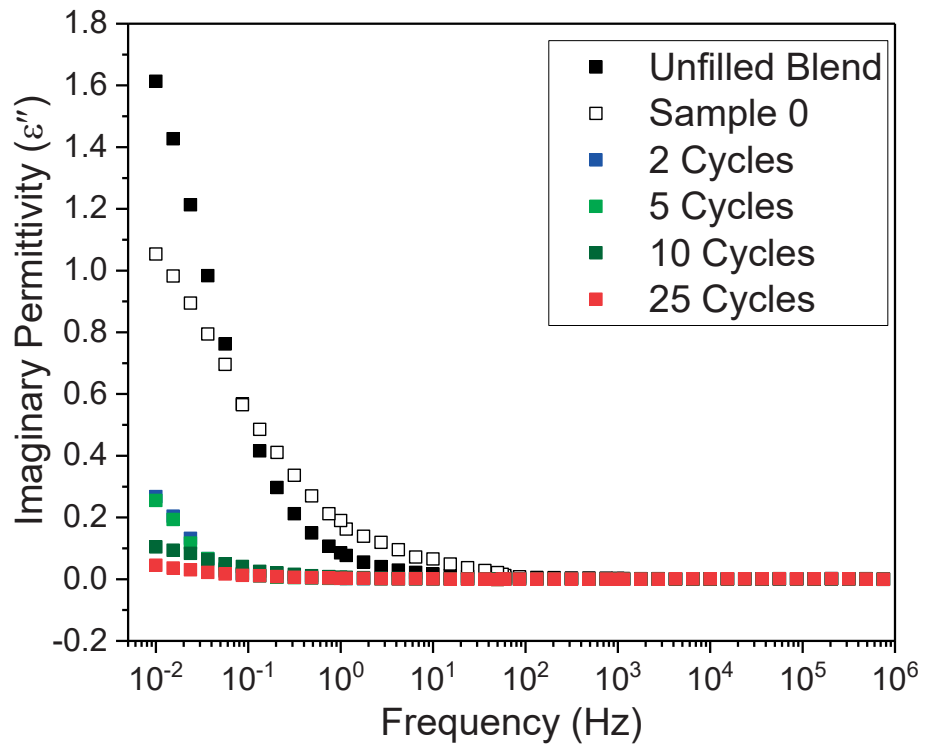

Figure 8.6. a: Real and b: imaginary parts of permittivity for the unfilled blend compared to NCs with modified and non-modified silica

The TSDC spectra of all the studied systems are presented in Figure 8.7a. The trap depth and density distributions are numerically calculated from the TSDC data, using a method reported by Tian et al. ${ }^{14}$, and presented in Figure $8.7 \mathrm{~b}$. The TSDC spectrum of the unfilled polymer blend exhibits a relaxation peak at $74{ }^{\circ} \mathrm{C}$, indicating the presence of traps with a distribution of depths around $0.98 \mathrm{eV}$ (see Figure $8.7 \mathrm{~b}$ ). These are likely charges 
released from the interfacial regions between the two polymer domains, and the crystalline/amorphous interfaces within either polymer phase. Upon incorporation of the untreated silica (Sample 0), the peak trap density appears at a slightly deeper level compared to the unfilled blend. The introduction of polyurea films to the NPs has a noticeable influence on the TSDC spectra. At 2 MLD cycles, a rather large relaxation peak appears at $63^{\circ} \mathrm{C}$, which can correspond to the existence of shallow trapping states with depths around $0.95 \mathrm{eV}^{14}$. Moreover, a smaller peak is observed at $\sim 105^{\circ} \mathrm{C}$ indicative of deeper states with energies around $1.07 \mathrm{eV}$. As the number of MLD cycles increases, the density of the deep traps increases, while the contribution of the shallow traps reduces. The DFT calculations predicted that there are contributions of both shallow and deep traps in the urea units under consideration. Also, the distribution of the molecular orbitals suggested that the phenyl rings and the amide groups are likely responsible for the shallow and deep traps, respectively. Therefore, the lower energy peak in the TSDC spectra of the modified NCs can be attributed to the relaxation of space charge trapped in the phenyl shallow states, whereas the high energy peak is due the relaxation of charges that occupy the amide deep traps. As the MLD film grows with increasing number of cycles, and the density of deep traps increases, the charges would be immobilized for longer periods of time. This would naturally result in a lower contribution of shallow traps, and consequently more space charge may be formed at the interfaces. This can clearly be seen in Figure 8.7c, where the amount of injected charge is minimum at 2 MLD cycles with the highest density of shallow traps. It is clear that the polarization step was too short to reach steady-state DC conduction in the samples; nevertheless, Figure 8.7e demonstrates that the NC with 2-cyclemodified silica exhibits the lowest apparent conductivity at the end of the poling step $\left(4.9 \times 10^{-13} \mathrm{~S} / \mathrm{m}\right)$ among all studied samples. 
a.

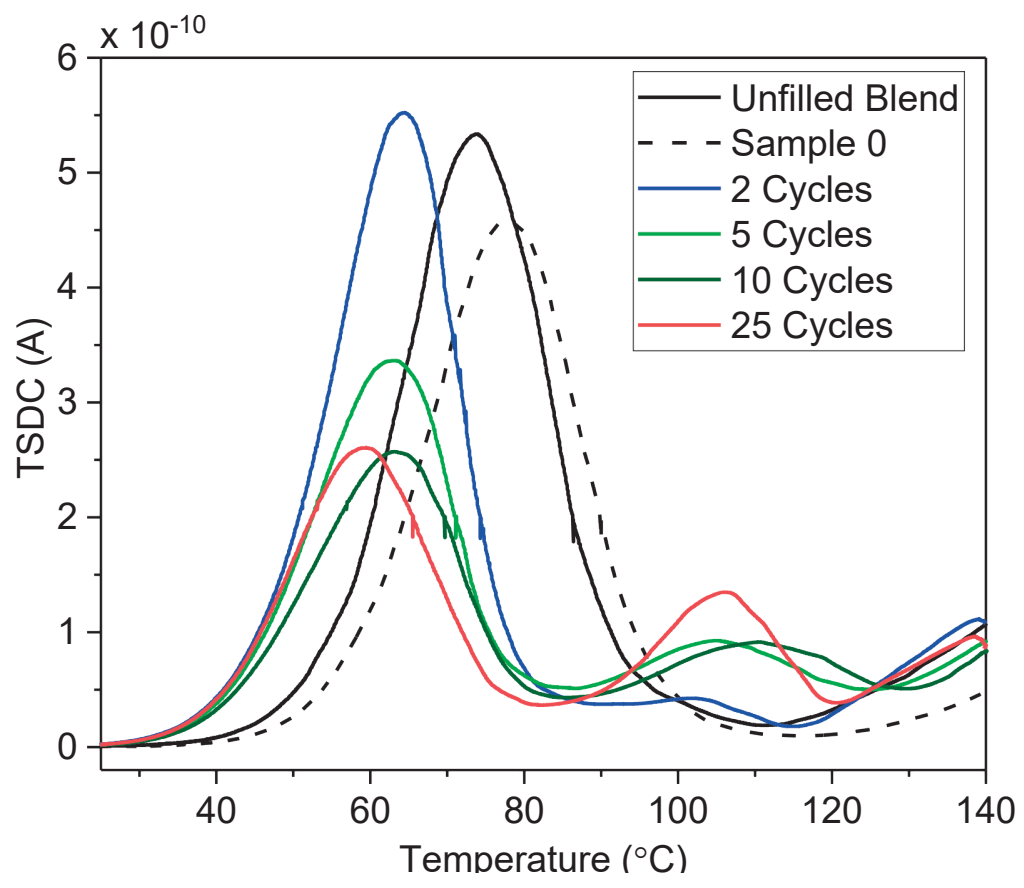

b.

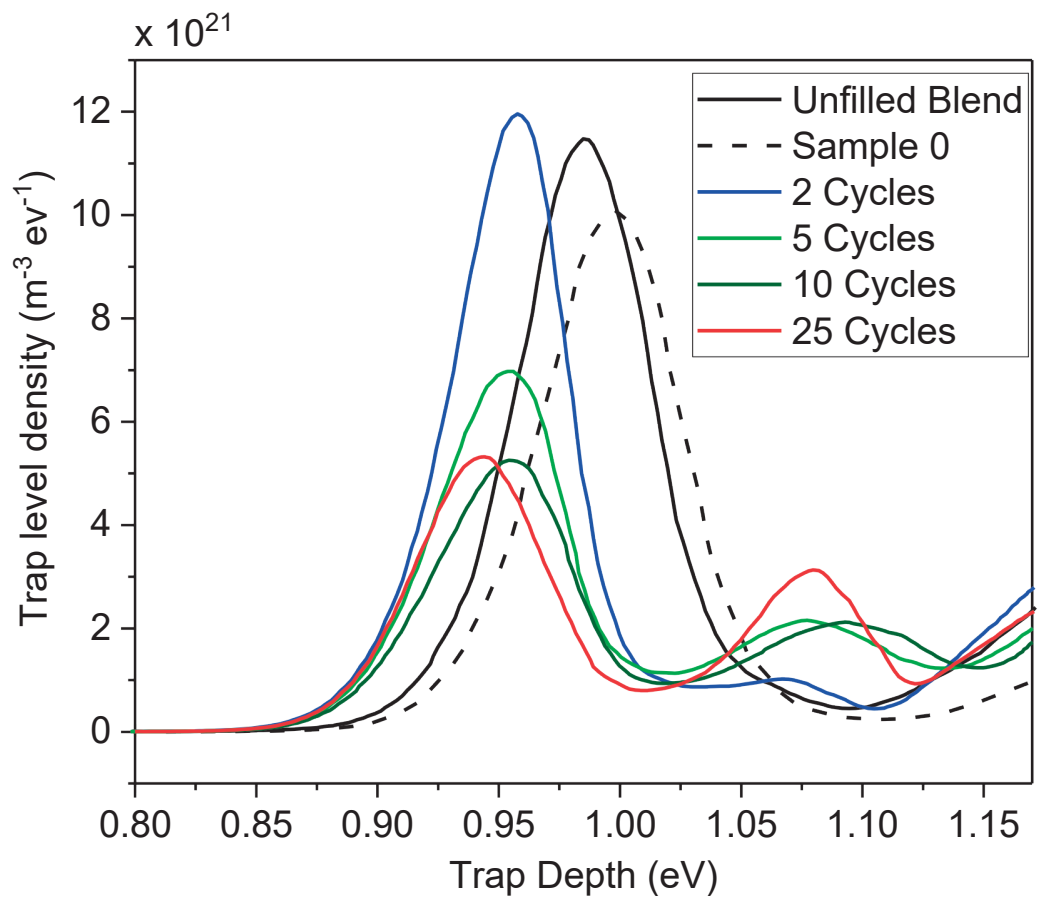


c.

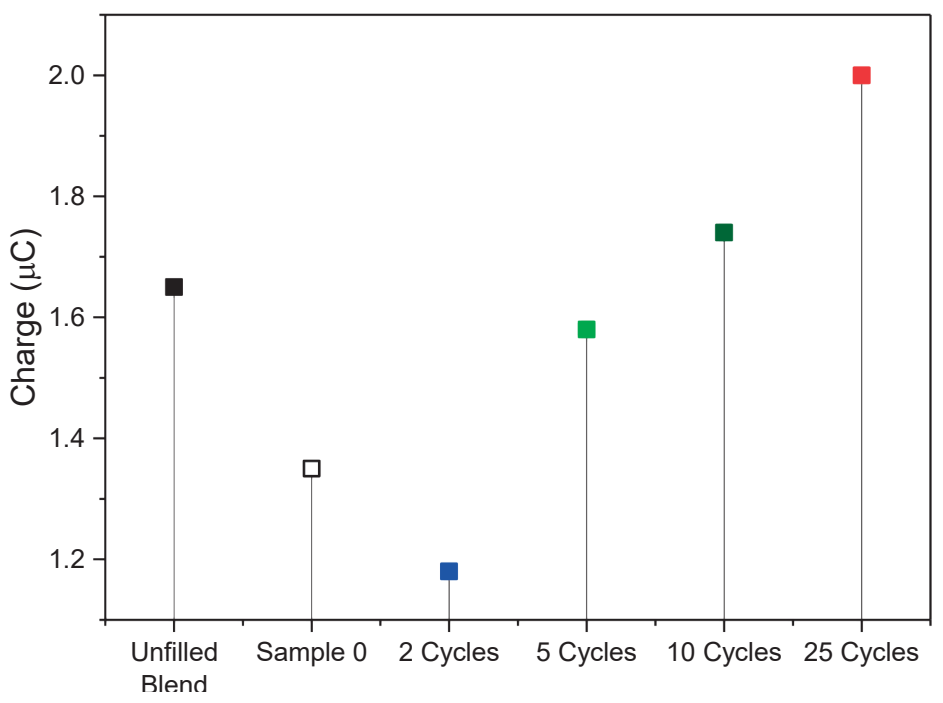

d.

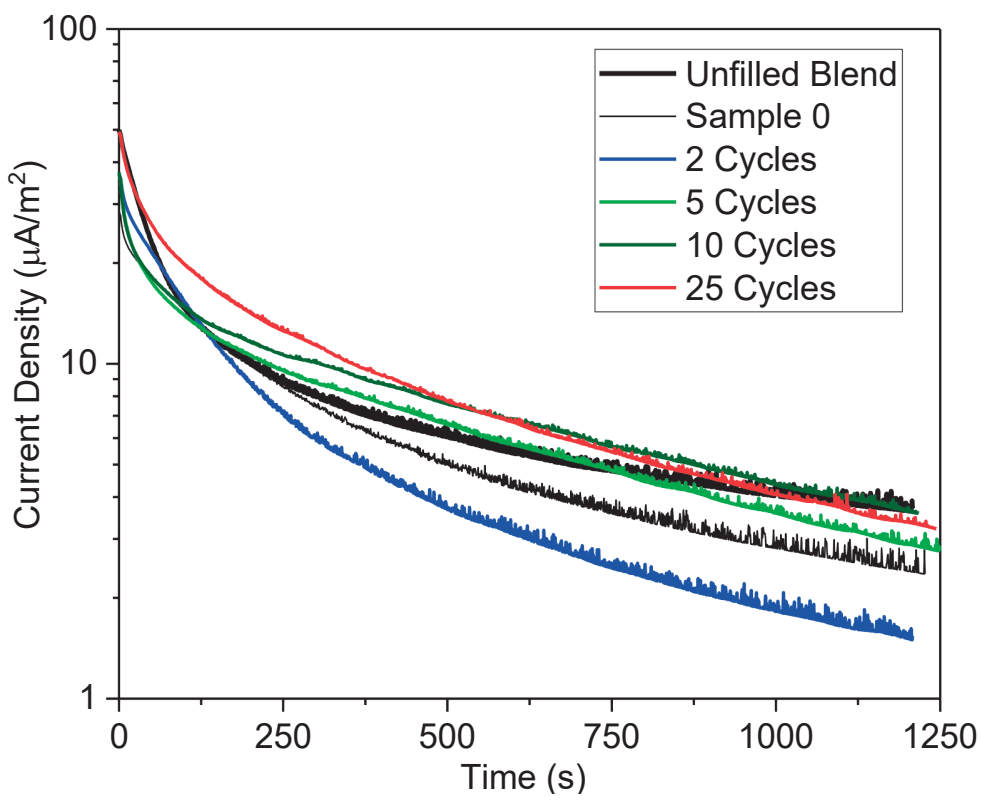


e.

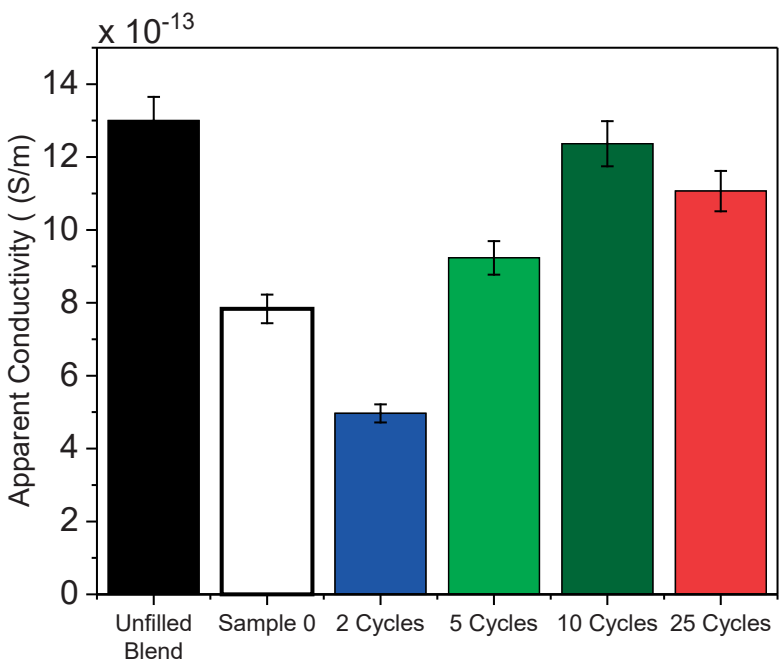

Figure 8.7. a: TSDC spectra, b: trap level distribution and density, c: amount of injected charge during poling, $\mathrm{d}$ : current density during poling and e: apparent conductivity at the end of poling for all NCs and the unfilled reference.

Evidently, by grafting a homogenous polyurea film onto the silica NPs, it is possible to create a bi-modal distribution of trap depths. The phenyl rings in the deposited film are able to introduce shallow trapping states that could reduce charge mobility, and hence hinder conduction processes. Instead, the amide groups are prone to create deeper states, which in an occupied state can hamper further formation of space charge at the filler-polymer interfaces. The introduction of deep traps by the nitrogen-containing functional groups was also observed in the case of the APTES- and ureido-modified silica, as shown in Chapters 3, 4 and 6 . The main advantage of the polyurea deposition is that it introduces both shallow and deep traps, the density of which is virtually controllable by the number of MLD cycles. Moreover, as shown in the DFT results, the second monomer in the urea unit (ED) can induce hole trap states that can further reduce the overall charge mobility in the NCs. The presence of all these newly introduced states at the filler-polymer interface makes this type of modification very suitable for developing dielectric NCs with superior insulation properties. 


\section{Conclusions}

In this study, we firstly demonstrated the electronic structure of silica upon deposition of polyurea films via molecular layer deposition (MLD). With the LUMO and HOMO levels being distributed over the phenyl diisocyanate (PDIC) and ethylene diamine (ED) monomers, respectively, the urea-modified silica exhibited a significantly different electronic structure compared to the bare NPs. This indicated that each monomer can play a different role in charge transport processes. Silica modification facilitated nucleation and crystal formation, and nanoparticle dispersion in the polymer matrix improved with higher grafting densities of polyurea. Dielectric measurements revealed that the polyurea deposition results in a bi-modal distribution of shallow and deep traps with densities dependent on the number of MLD cycles. In particular, with increasing number of cycles, the density of the deep traps increases, whereas that of shallow traps decreases. The large density of valence states related to the amino functional groups in the polyurea film hampered the formation of space charge, and the shallow trapping states corresponding to the phenyl rings resulted in a lower current density under a DC electric field. Therefore, MLD offers a reliable tool for controlling the density of shallow and deep traps in nanodielectrics, which can be of utmost importance in developing insulating materials with low space charge accumulation and conductivity.

\section{References}

[1] George, S. M.; Yoon, B.; Dameron, A. A., Surface Chemistry for Molecular Layer Deposition of Organic and Hybrid Organic-Inorganic Polymers. Accounts of Chemical Research, 42 (4), 2009, pp 498-508.

[2] Du, Y.; George, S., Molecular layer deposition of nylon 66 films examined using in situ FTIR spectroscopy. The Journal of Physical Chemistry C, 111 (24), 2007, pp 8509-8517.

[3] Kubono, A.; Yuasa, N.; Shao, H.-L.; Umemoto, S.; Okui, N., In-situ study on alternating vapor deposition polymerization of alkyl polyamide with normal molecular orientation. Thin Solid Films, 289 (1-2), 1996, pp 107-111.

[4] La Zara, D.; Bailey, M. R.; Hagedoorn, P.-L.; Benz, D.; Quayle, M. J.; Folestad, S.; van Ommen, J. R., Sub-nanoscale Surface Engineering of TiO2 Nanoparticles by Molecular Layer Deposition of Poly(ethylene terephthalate) for Suppressing Photoactivity and Enhancing Dispersibility. ACS Applied Nano Materials, 2020. 
[5] Gil-Font, J.; Hatte, M.-A.; Bailey, M. R.; Navarrete, N.; Ventura-Espinosa, J.; Goulas, A.; La Zara, D.; van Ommen, J. R.; Mondragón, R.; Hernández, L., Improving heat transfer of stabilised thermal oil-based tin nanofluids using biosurfactant and molecular layer deposition. Applied Thermal Engineering, 178, 2020, p 115559.

[6] Van Bui, H.; Grillo, F.; Van Ommen, J., Atomic and molecular layer deposition: off the beaten track. Chemical Communications, 53 (1), 2017, pp 45-71.

[7] Zhou, Y.; Hu, J.; Dang, B.; He, J., Titanium oxide nanoparticle increases shallow traps to suppress space charge accumulation in polypropylene dielectrics. RSC Advances, 6 (54), 2016, pp 48720-48727.

[8] Wang, W.; Min, D.; Li, S., Understanding the conduction and breakdown properties of polyethylene nanodielectrics: effect of deep traps. IEEE Transactions on Dielectrics and Electrical Insulation, 23 (1), 2016, pp 564-572.

[9] Mahtabani, A.; Rytöluoto, I.; Anyszka, R.; He, X.; Saarimäki, E.; Lahti, K.; Paajanen, M.; Dierkes, W.; Blume, A., On the Silica Surface Modification and Its Effect on Charge Trapping and Transport in PP-Based Dielectric Nanocomposites. ACS Applied Polymer Materials, 2 (8), 2020, pp 3148-3160.

[10] He, X.; Rytöluoto, I.; Anyszka, R.; Mahtabani, A.; Saarimäki, E.; Lahti, K.; Paajanen, M.; Dierkes, W.; Blume, A., Silica Surface-Modification for Tailoring the Charge Trapping Properties of PP/POE Based Dielectric Nanocomposites for HVDC Cable Application. IEEE Access, 8, 2020, pp 87719-87734.

[11] Binsbergen, F. L., Heterogeneous nucleation in the crystallization of polyolefins: Part 1. Chemical and physical nature of nucleating agents. Polymer, 11 (5), 1970, pp 253267.

[12] Nelson, J. K.; Fothergill, J. C., Internal charge behaviour of nanocomposites. Nanotechnology, 15 (5), 2004, p 586.

[13] Nelson, J. K., Dielectric polymer nanocomposites Springer: 2010;

[14] Tian, F.; Bu, W.; Shi, L.; Yang, C.; Wang, Y.; Lei, Q., Theory of modified thermally stimulated current and direct determination of trap level distribution. Journal of Electrostatics, 69 (1), 2011, pp 7-10. 



\section{CHAPTER 9}

Summary 
Studying nanometric dielectrics is a $21^{\text {st }}$ century phenomenon. They have come a long way. A scientific journey that started around 25 years ago and is been maturing ever since. Yet, despite the large volume of research devoted to them so far, there are still plenty of unknowns to be unraveled. Particularly, the role of nanometric interfaces within these materials induced by the nano-sized filler dispersed in the polymeric matrix, and the mechanisms through which they affect the overall dielectric properties of the system are still far from being fully understood.

As an effort to shed some light on these blind spots, the present work focuses on the role of the nanofiller and its surface properties. Altering the surface chemistry of nanoparticles could potentially change the filler-polymer interfacial interactions in nanocomposites, leading to significant improvements of the dielectric performance of the material under electric fields. This has been the main theme of this thesis. Fumed silica nanoparticles, a highly pure member of the silica family, were used together with polypropylene (PP)/ ethylene-octene-copolymer (EOC) blends constituting the nanocomposite systems under investigation. A conventional liquid-phase and two gasphase methods were utilized for the treatment of the nanoparticles' surface.

Using the liquid-phase modification technique, 3-aminopropyltriethoxysilane (APTES) was grafted onto the silica nanoparticles. It was shown that the amino functional group in the APTES structure introduces deep trapping states to the filler-polymer interface, that effectively reduce the injection of charge into the dielectric under direct current (DC) electric fields and mitigate the formation of space charge at the interfaces. In the next step, other functional groups, namely ureido and methacrylate moieties, were deposited onto the nanoparticles, and the dielectric performance of the resulting nanocomposites were put to test. On the one hand, the nitrogen containing ureido groups introduced deep localized states and hampered the space charge formation, similar to what was observed in the case of APTES modification. On the other hand, the methacrylate moiety improved the dispersion of the nanoparticles in the polymer matrix; nevertheless, the space charge formation was not reduced as significantly as in the case of ureido functionalization. 
These observations led us to the next step to take a closer look at the electronic structure of the silica surface before and after modification with the nitrogen containing APTES precursor. This was done by means of density functional theory (DFT), which determines the electronic band structure of matter by solvation of the time-independent Schrödinger's equation for a given chemical entity. The DFT results revealed that the aminofunctionalized silica does contain deep localized states and exhibits a different electron/hole distribution compared to the untreated nanoparticle. To validate the DFT model predictions, nanoparticles were modified with APTES via a novel gas-phase method similar to atomic layer deposition (ALD) in a fluidized bed. Utilizing this method, it was possible to deposit self-limiting layers of the precursor onto the nanoparticles in a controlled way, using consecutive APTES/ $\mathrm{H}_{2} \mathrm{O}$ cycles in vapor phase. Dielectric tests on the nanocomposites revealed the presence of deep traps, similar to what was observed in case of the solution modified silica. There was also an inverse correlation between the number of APTES/ $\mathrm{H}_{2} \mathrm{O}$ cycles and the density of the occupied deep traps in the nanocomposite systems. The reduction of the occupied deep trap density resulted in lower space charge formation at the interfaces. One direct implication of this is that one may control the density of the introduced deep traps, and therefore, space charge formation in nanocomposites, by altering the number of APTES/ $\mathrm{H}_{2} \mathrm{O}$ cycles during silica modification.

The results of the fluidized bed modification were intriguing enough for us to try another gas-phase method for silica modification: molecular layer deposition (MLD). In this part of the work, self-limiting layers of polyurea were deposited on the silica nanoparticles using phenyldiisocyanate (PDIC) and ethylenediamine (ED) as precursors, via consecutive PDIC/ED cycles passing through the fluidized silica. The resulting urea units contain amide and phenyl functional groups which can potentially introduce localized states at the fillerpolymer interfaces in nanocomposites. The DFT method was also utilized here, and the results showed that this urea unit exhibits the contribution of both shallow and deep traps. Dielectric tests on the nanocomposites validated this prediction. A bi-modal distribution of shallow and deep traps was observed in the nanocomposites, associated with the phenyl and amide groups, respectively. With increasing the number of PDIC/ED cycles, the density of the deep traps increased, whereas that of shallow traps decreased. The large density of 
valence states related to the amide functional groups in the polyurea film hampered the formation of space charge, and the shallow trapping states corresponding to the phenyl rings resulted in a lower current density under a DC electric field. Therefore, MLD offers a reliable tool for controlling the density of shallow and deep traps in nanodielectrics, which can be of utmost importance in developing insulating materials with low space charge accumulation and conductivity.

All the results discussed in this thesis point towards an important conclusion: that charge trapping and transport as well as space charge phenomena in nanocomposites can be controlled by altering the electronic structure of the filler. This can be explained using Lewis's formulation of the interfacial interactions in nanodielectrics ${ }^{1}$. According to this model, space charge and conductivity are largely affected by the electric double layer that is superimposed near the filler-polymer interfaces in the presence of an electric field. The electric double layer contains mobile charge carriers that are partly under the influence of the nanoparticles' surface charge. Therefore, the observed improvements can be attributed to the alterations of the surface charge and electronic states of the nanoparticles.

As mentioned in the beginning of this summary, the interfacial phenomena in nanodielectrics still lack fundamental understanding. In the present work, we addressed some interesting features of this class of materials by focusing on the nanoparticles and their surface chemistry. On the path towards a more sustainable energy transmission, and in the spirit of the super-grid concept introduced in the beginning of this thesis, the importance of high performance cables is undeniable. The observations discussed here, point towards materials and methods that can be utilized to take significant steps towards this ultimate goal.

Nevertheless, still plenty of room remains for exploration and scientific inquiry. Further analysis of thermal conductivity, breakdown strength, partial discharge and DC conductivity are among the most important factors to take into account for the next steps. Furthermore, DFT modelling shows promising potential to predict and explain some of the interfacial phenomena in nanodielectrics. However, not much attention has been paid to this aspect of analysis so far. Although the current limited computational power is a big 
drawback in this manner, with the development of new generations of computers, it can be feasible in the near future to take bigger steps towards a full realization of nanodielectrics' potential.

\section{References}

[1] Lewis, T., Interfaces: nanometric dielectrics. Journal of Physics D: Applied Physics, 38 (2), 2005, p 202. 


\section{Samenvatting}

Onderzoek naar nanodiëlektrica is opgekomen in de $21^{\text {ste }}$ eeuw en heeft een lange weg afgelegd: Een wetenschappelijke reis die ongeveer 25 jaar geleden begon en sindsdien verder is ontwikkeld. Maar ondanks het grote aantal studies dat er tot dusver aan is besteed, zijn er tal van onbekende factoren die nog moeten worden ontrafeld. Met name de rol van nanometrische grensvlakken tussen polymere matrices en nano-vulstoffen, en de mechanismen waardoor deze de diëlektrische eigenschappen van composieten beïnvloeden, worden nog verre van volledig begrepen.

Om enig licht te werpen op deze blinde vlekken, concentreert het huidige werk zich op de rol van de nanovulstof en zijn oppervlakte-eigenschappen. Modificatie van de oppervlakte chemie van nanodeeltjes zou de interacties in het grensvlak tussen vulstof en polymere matrix kunnen beïnvloeden en leiden tot aanzienlijke verbeteringen van de diëlektrische eigenschappen van het materiaal in een elektrisch veld. Dit is de belangrijkste onderzoeksvraag van dit proefschrift. Pyrogene silica nanodeeltjes, een zeer zuivere vorm van silica, en een blend van polypropeen (PP)/etheen-octeen-copolymeer (EOC) zijn de bestanddelen van het onderzochte nanocomposiet. Een conventionele modificatie in vloeistof en twee gasfase methoden werden gebruikt voor de modificatie van het oppervlak van de nanodeeltjes.

Met behulp van de vloeistoffase-modificatietechniek werd 3aminopropyltriethoxysilaan (APTES) op de silica-nanodeeltjes geënt. Er werd aangetoond dat de amino-groep in de APTES-structuur diepe 'traps' introduceert in het silica/polymeer grensvlak, die de ladingsinjectie in het diëlektricum onder gelijkstroom (DC) elektrische velden effectief verminderen en de vorming van ruimtelijk verdeelde lading (ruimtelading) reduceren. In de volgende stap werden andere functionele groepen, namelijk ureido- en methacrylaat-groepen, aangebracht op de nanodeeltjes, en vervolgens de diëlektrische eigenschappen van het resulterende nanocomposiet onderzocht. Enerzijds introduceren de stikstof-bevattende ureido-groepen diepe gelocaliseerde niveaus en reduceren het ontstaan van ruimtelading, vergelijkbaar met de effecten waargenomen bij APTES modificatie. Anderzijds verbeterden de methacrylaat- 
groepen de dispersie van de nanodeeltjes in de polymeermatrix; daarnaast was de vorming van ruimtelading in mindere mate verlaagd vergeleken met ureido-functionalisatie van silica.

Deze waarnemingen leidden ons naar de volgende stap om de elektronische structuur van het silica-oppervlak vóór en na modificatie met de stikstofhoudende APTES-precursor nader te onderzoeken. Daarvoor werd gebruik gemaakt van de 'density functional theory' (DFT), die de elektronische bandstructuur van materie bepaalt door oplossen van de tijdonafhankelijke Schrödinger-vergelijking voor een gegeven chemische entiteit. De DFTresultaten toonden aan dat het amino-gefunctionaliseerde silica diepe gelokaliseerde 'traps' bevat en een andere elektronengat-verdeling vertoont in vergelijking met de niet gemodificeerde nanodeeltjes. Om de voorspellingen van het DFT-model te valideren, werden silica deeltjes gemodificeerd met APTES via een nieuwe gasfase methode, vergelijkbaar met 'Atomic Layer Deposition' (ALD) in een wervelbed reactor. Zo was het mogelijk om zelfbeperkende lagen van de precursor op een gecontroleerde manier op de nanodeeltjes aan te brengen door opeenvolgende APTES/ $\mathrm{H}_{2} \mathrm{O}$-cycli in de dampfase. Diëlektrische testen van de nanocomposieten lieten de aanwezigheid van diepe 'traps' zien, vergelijkbaar met wat werd waargenomen in het geval van in oplossing gemodificeerde silica. Er werd een omgekeerde correlatie tussen het aantal APTES/ $\mathrm{H}_{2} \mathrm{O}-$ cycli en de dichtheid van de bezette diepe 'traps' in de nanocomposiet systemen gevonden. De vermindering van de bezette diepe 'trap' dichtheid resulteerde in een verlaagde vorming van ruimtelading in de grensvlakken. Een direct gevolg hiervan is dat de dichtheid van geïntroduceerde diepe 'traps' en dus het ontstaan van ruimtelading gecontroleerd kan worden door het aantal APTES/ $\mathrm{H}_{2} \mathrm{O}$ cycli tijdens silica modificatie.

De resultaten van de wervelbedmodificatie waren intrigerend genoeg om nog een andere gasfase methode voor silica-modificatie uit te voeren: 'Molecular Layer Deposition' (MLD). In dit deel van de studie werden zelfbeperkende lagen polyureum op silica nanodeeltjes opgebracht via opeenvolgende fenyldiisocyanaat (PDIC)/ethyleendiamine (ED) cycli in een wervelbed van silica. De resulterende ureumlaag bevatte amide en fenyl groepen welke gelocaliseerde 'trap' niveaus kunnen vormen in het vulstof/polymeer 
grensvlak van het nanocomposiet. Ook hier werd de DFT-methode gebruikt, en de resultaten toonden aan dat deze ureumgroep zowel ondiepe als diepe 'traps' introduceert. Deze vinding is door diëlektrische tests op de nanocomposieten bevestigd. Een bimodale verdeling van ondiepe en diepe 'traps' in de nanocomposieten werd geassocieerd met respectievelijk de fenyl- en amidegroepen. Naarmate het aantal PDIC/ED-cycli toenam, nam de dichtheid van de diepe 'traps' ook toe, terwijl die van ondiepe 'traps' afnam. De hoge dichtheid van valentie-toestanden die verband houden met de functionele amidegroepen in de polyureum film verlaagde de vorming van ruimtelading, en de ondiepe 'traps' die gevormd worden door fenylringen resulteerden in een lagere stroomdichtheid in een elektrisch DC veld. Daarom mag gesteld worden dat MLD een betrouwbare methode is voor het controleren van de dichtheid van ondiepe en diepe 'traps' in nanodiëlektrica, wat van het grootste belang kan zijn bij het ontwikkelen van isolatiematerialen met een lage ruimteladingsverdeling en elektrische geleiding.

Alle resultaten van de studie binnen dit proefschrift wijzen op een belangrijke conclusie: ladingslocalisatie, ladings-transport en ruimteladingsfenomenen in nanocomposieten kunnen worden gecontroleerd door de elektronische structuur van de vulstof. Dit kan worden verklaard met de definitie van Lewis van grensvlakinteracties in nanodiëlektrica [1]. Volgens dit model worden ruimtelading en geleiding grotendeels beïnvloed door de elektrische dubbellaag nabij het vulstof/polymeer grensvlak in aanwezigheid van een elektrisch veld. De elektrische dubbellaag bevat mobiele ladingsdragers die gedeeltelijk beïnvloed worden door de oppervlaktelading van de nanodeeltjes. Daarom kunnen de waargenomen verbeteringen worden toegeschreven aan de veranderingen van de oppervlaktelading en elektronische structuur van de nanodeeltjes.

Zoals gesteld in het begin van deze samenvatting, ontbreekt het nog steeds aan fundamenteel begrip van grensvlakverschijnselen in nanodiëlektrica. In de huidige studie zijn enkele kenmerken van deze materialen ontrafeld door diepgaand onderzoek naar de nanodeeltjes en hun oppervlaktechemie. Langs de weg naar duurzamer energie-transport, en in de geest van het super-grid concept geïntroduceerd in het begin van dit proefschrift, spreekt het belang van hoogwaardige kabels vanzelf. De waarnemingen bediscussieerd in 
dit proefschrift wijzen in de richting van materialen en methodes welke gebruikt kunnen worden om significante stappen voorwaarts te maken naar het uiteindelijke doel.

Desalniettemin is er nog veel wetenschappelijk onderzoek nodig om deze nanodiëlektrische composieten helemaal te begrijpen. Verdere analyse van thermische geleidbaarheid, elektrische doorslagsterkte, gedeeltelijke ontlading en DC-geleidbaarheid behoren tot de belangrijkste factoren waarop men zich zou moeten richten bij volgende stappen. Bovendien is DFT-modellering veelbelovend voor de voorspelling en verklaring van enkele van de grensvlakverschijnselen in nanodiëlektrica. Evenwel, niet veel aandacht is geschonken aan dit analyse-aspect en viel buiten de reikwijdte van de huidige studie. Hoewel de huidige beperkte rekenkracht een groot nadeel is, kunnen door de ontwikkeling van nieuwe generaties computers in de nabije toekomst grotere stappen gezet worden in de richting van gebruik van het volledige potentieel van nanodiëlektrica. 


\title{
List of Publications
}

\author{
Journal Articles:
}

1. Mahtabani, A., Rytöluoto, I., Anyszka, R., He, X., Saarimäki, E., Lahti, K., Paajanen, M., Dierkes, W., and Blume, A., "On the Silica Surface Modification and Its Effect on Charge Trapping and Transport in PP-Based Dielectric Nanocomposites", ACS Applied Polymer Materials, vol. 2, no. 8, pp. 3148-3160, 2020.

2. Mahtabani, A.; La Zara, D.; Anyszka, R.; He, X.; Paajanen, M.; van Ommen, J. R.; Dierkes, W.; Blume, A., "Gas Phase Modification of Silica Nanoparticles in a Fluidized Bed: Tailored Deposition of Aminopropylsiloxane", Langmuir, 37 (15), 2021, pp 44814492.

3. Mahtabani, A.; Niittymäki, M.; Anyszka, R.; Rytöluoto, I.; He, X.; Saarimäki, E.; Lahti, K.; Paajanen, M.; Dierkes, W.; Blume, A., "Deposition of Ureido and Methacrylate Functionalities Onto Silica Nanoparticles and its Effect on the Properties of Polypropylene-Based Nanodielectrics" IEEE Access, 9, 2021, pp 130340-130352.

4. Mahtabani, A.; La Zara, D.; Niittymäki, M.; Anyszka, R.; Rytöluoto, I.; He, X.; Saarimäki, E.; Lahti, K.; Paajanen, M.; Dierkes, W.; Blume, A. "Surface Modified Silica Nanoparticles in PP Based Nanodielectrics: Electronic Structure of and Its Effect on Charge Transport", Journal of Physics and Chemistry of Solids, under review.

5. Mahtabani, A.; La Zara, D.; Niittymäki, M.; Anyszka, R.; Rytöluoto, I.; He, X.; Saarimäki, E.; Lahti, K.; Paajanen, M.; Dierkes, W.; Blume, A., "Dielectric properties of PP Based Nanocomposites: Effect of Molecular Layer Deposition of Polyurea on Silica Nanoparticles", Journal of Physical Chemistry C, under review.

\section{Conference Articles:}

1. Mahtabani, A., He, X., Rytöluoto, I., Lahti, K., Paajanen, M., Saarimäki, E., Anyszka, R., Dierkes, W., and Blume, A., "Effect of silica modification on charge trapping behavior of PP blend/silica nanocomposites", in 2019 2nd International Conference on Electrical Materials and Power Equipment (ICEMPE), 2019, pp. 241-245, IEEE.

2. Mahtabani, A.; Rytöluoto, I.; He, X.; Saarimäki, E.; Lahti, K.; Paajanen, M.; Anyszka, R.; Dierkes, W.; Blume, A. "Solution modified fumed silica and its effect on charge trapping behavior of PP/POE/silica nanodielectrics", Nordic Insulation Symposium: 2019. 
3. Mahtabani, A.; Rytöluoto, I.; Anyszka, R.; Saarimäki, E.; He, X.; Lahti, K.; Paajanen, M.; Dierkes, W.; Blume, A. Silica Functionalization: "How Does it Affect Space Charge Accumulation in Nanodielectrics Under DC?", 2020 IEEE 3rd International Conference on Dielectrics (ICD), 2020; pp 281-284, IEEE.

\section{Collaborations:}

1. He, X.; Rytöluoto, I.; Anyszka, R.; Mahtabani, A.; Saarimäki, E.; Lahti, K.; Paajanen, M.; Dierkes, W.; Blume, A., "Surface Modification of Fumed Silica by Plasma Polymerization of Acetylene for PP/POE Blends Dielectric Nanocomposites". Polymers, 11 (12), 2019, p 1957.

2. He, X.; Rytöluoto, I.; Anyszka, R.; Mahtabani, A.; Saarimäki, E.; Lahti, K.; Paajanen, M.; Dierkes, W.; Blume, A., "Silica Surface-Modification for Tailoring the Charge Trapping Properties of PP/POE Based Dielectric Nanocomposites for HVDC Cable Application". IEEE Access, 8, 2020, pp 87719-87734.

3. He, X.; Seri, P.; Rytöluoto, I.; Anyszka, R.; Mahtabani, A.; Naderiallaf, H.; Niittymäki, M.; Saarimäki, E.; Mazel, C.; Perego, G.; Lahti, K.; Paajanen, M.; Dierkes, W.; Blume, A., "Dielectric Performance of Silica-Filled Nanocomposites Based on Miscible (PP/PPHI) and Immiscible (PP/EOC) Polymer Blends". IEEE Access, 9, 2021, pp 15847-15859.

4. He, X.; Rytöluoto, I.; Seri, P.; Anyszka, R.; Mahtabani, A.; Naderiallaf, H.; Niittymäki, M.; Saarimäki, E.; Mazel, C.; Perego, G.; Lahti, K.; Paajanen, M.; Dierkes, W.; Blume, A., "PP/PP-HI/silica nanocomposites for HVDC cable insulation: Are silica clusters beneficial for space charge accumulation?" Polymer Testing, 98, 2021, p 107186.

5. He, X.; Mahtabani, A.; Rytöluoto, I.; Saarimäki, E.; Lahti, K.; Paajanen, M.; Anyszka, R.; Dierkes, W.; Blume, A. "Surface Modification of Fumed Silica by Dry Silanization for PP-based Dielectric Nanocomposites", 2019 2nd International Conference on Electrical Materials and Power Equipment (ICEMPE), pp 254-259, IEEE.

6. He, X.; Rytöluoto, I.; Mahtabani, A.; Saarimäki, E.; Lahti, K.; Paajanen, M.; Anyszka, R.; Dierkes, W.; Blume, A. "Surface Modification of Fumed Silica by Dry Silanization for PP/POE-based Dielectric Nanocomposites", Proceedings of the Nordic Insulation Symposium, 2019; pp 32-36.

7. He, X.; Seri, P.; Rytöluoto, I.; Anyszka, R.; Mahtabani, A.; Naderiallaf, H.; Saarimäki, E.; Niittymäki, M.; Mazel, C.; Perego, G.; Lahti, K.; Paajanen, M.; Dierkes, W.; Blume, A. "Influence of polar and unpolar silica functionalization on the dielectric properties 
of PP/POE nanocomposites", 2020 IEEE 3rd International Conference on Dielectrics (ICD), 2020; pp 229-232, IEEE.

8. Anyszka, R.; He, X.; Mahtabani, A.; Dierkes, W. K.; Blume, A. "Effective surface functionalization of fumed silica with NBR telechelic oligomer for high-voltage polyolefin-based dielectric composites", Proceedings of the Nordic Insulation Symposium, 2019; pp 118-122.

9. Montanari, G. C.; Seri, P.; Paajanen, M.; Lahti, K.; Rytöluoto, I.; Ritamäki, M.; Blume, A.; Dierkes, W.; Mahtabani, A.; He, X. "Potentiality of nanofilled thermoplastic insulation for DC cables and capacitors", 2018 IEEE 2nd International Conference on Dielectrics (ICD), 2018; pp 1-4, IEEE.

10. Montanari, G. C.; Seri, P.; Naderiallaf, H.; Blume, A.; Mahtabani, A.; He, X.; Perego, G.; Mazel, C.; Paajanen, M.; Karttunen, M. "Adding nanofillers in polymeric insulating materials: so far so good? The case of polypropylene for DC cables", 2019 IEEE Electrical Insulation Conference (EIC), IEEE: 2019; pp 477-480, IEEE.

11. Blume, A.; Jin, J.; Mahtabani, A.; He, X.; Kim, S.; Andrzejewska, Z. "New Structure Proposal for Silane Modified Silica", International Rubber Conference, IRC 2019, London, 2019. 


\section{Acknowledgments}

"When you reach the end of what you should know, you will be at the beginning of what you should sense."

- Gibrán Kahlil Gibrán, Sand and Foam

I would like to dedicate this part of the thesis to the efforts of others involved in assembling this small scientific endeavor, piece by piece. Writing a thesis and getting a PhD may seem like solitary activities, but in truth, they are not. This is an extraordinary experience for the fellow science enthusiast, which can easily turn into a painful disaster without the assistance of people who care. I would like to start by expressing my gratitude to my supervisors: Prof. Anke Blume, and Dr. Wilma Dierkes.

Dear Anke, thank you for giving me the opportunity to join the ETE group, and start this journey in the first place. I will always be grateful for that. I had the pleasure to spend more time with you in the beginning of the project, which was of great help, especially as everything looked very scary to me at the time. Thank you for your professional and mental support during these years.

Dear Wilma, it is very clear to me that I could not have possibly reached the point of writing these words right now, without your support and supervision during the last four and a half years. Thank you for guiding me in the way of learning the scientific method, correcting me when I was wrong, acknowledging my point of view when I had little faith, and your constant support during all the challenges that came through. You are a great mentor to me, and will always remain one of my favorite scientists. I look forward to staying in touch, and maybe working with you again in one way or another in the future.

I had the privilege to be recipient of scientific advice from Prof. Jacques Noordermeer, who is definitely one of the greatest scientists in the field of elastomer (rubber) technology. It is impossible to have a conversation with him, and not learn something. Thank you Jacques, for your invaluable input to this work.

I pursued my PhD in the framework of the GRIDABLE project, a combination of real professionals from both academia and industry. Working alongside them was an immensely significant experience 
for me. A massive thank you to all for your input to this work and to the project along the way. Special thanks to Dr. Mika Paajanen who coordinated this project in the best way possible, and supported us every step of the way. I would like to express my appreciation to Dr. Ilkka Rytöluoto and Eetta Saarimäki from VTT research center of Finland for assisting us in the preparation of samples, and a lot more. Ilkka was in fact, my gateway into the dielectric phenomena. We had many interesting and insightful discussions over the years which gave me a lot of ideas for approaching my research; I will always be grateful for that. I would like to thank Dr. Paolo Seri of University of Bologna, who helped us greatly with space charge and conductivity measurements, and his insights into dielectric materials. A warm gratitude goes to Dr. Kari Lahti and Minna Niittymäki from Tampere University who helped us tremendously with the TSDC measurements. This work could not be done without you generously endowing us with your time, insight and of course your High Voltage Engineering lab which I had the pleasure to visit in 2018.

I would like to acknowledge the inputs of Prof. Ruud van Ommen, and Dr. Damiano La Zara from Delft University of Technology. Dear Ruud, thank you for giving me the opportunity to visit and work in your lab, and teaching me the principles of atomic and molecular layer deposition. Dear Damiano, thank you for all the hard work you did helping me performing the experiments, troubleshooting and many, many discussions. I learned quite a lot about these processes by working with you, and I am grateful for that.

A very special acknowledgment to Dr. Rafał Piotr Anyszka and Dr. Xiaozhen He, who were with me all along this trajectory. Doctor! It was an honor to do SCIENCE with you. You are the defenition of a cool scientist, and a good friend. Xiaozhen, I will really miss working with you at our back to back desk setting. You were always positive and motivating, and always pushing me towards improvement. I hope our friendship would thrive in time, more than before. This is a good chance to mention my other office mates: Arqam (Amir from Pakistan) and the center of the solar system Sun, with whom I shared room W255 as well as so many great memories.

And the list goes on! I am talking about the assistance of others. This assistance, is not solely professional and scientific. It can move through different fields and take many pleasant forms: mental support, friendship, companionship, coffee breaks, small talks in the morning, heavy discussions at lunch, dry jokes on the way back, Sony TVs, that pole someone ran into one time, cows on Mars, ordering toluene, Eddie, stack of noodles in the desk drawer, confusing plots for defense movies, Exploding Kittens, fresh air, being on a horse, beer after gym, random screams from the office across the hall and so on and on! 
I believe, my dear friends and colleagues, that you smiled at least to one of the fields I mentioned above. If that is the case, it means that you were an important part of my last 4 years, and I appreciate you, for just simply being there. Thank you!

I cannot go on without mentioning Dries (also known as Peter), and Ceciel who were always available to help and to solve our daily problems. My warmest gratitude to you both.

And here is to my dear paranymphs, Marzieh and Neven, and my dadach Ayush who are more than friends. They are my paranymphs for life. Marzieh, I thank you as many as the number of gol rizooli's you have ever seen, and I know that would not be even close to be enough. Ayush and Neven, please know that my life is a lot better than "sometimes maybe good", with you in it. So, keep texting!

And last but not at all least, I would like to thank my family. My mother to whom I owe everything. All I have ever achieved stands on the shoulders of this brave woman. My sister and brother Dr. Aidin and Dr. Amin Mahtabani with whom I share the fondest memories, and from whom I receive constant love and care, unconditionally. I will never forget the day of my flight from Iran to the Netherlands. They drove me half way across the country to the airport during one of the ridiculously harsh blizzards I have ever experienced. Everything about that day was extreme, including the love I felt from them. This last sentence would be in Persian and in praise of my family who stood by me my whole life:

$$
\text { عزيزاى دلم دوستتون دارم. مرسى واسه همه جى. دلم خيلى براتون تنَ شده. عشقيد، عشق! }
$$


